\author{
UNIVERSIDADE DE SÃO PAULO \\ ESCOLA DE ENGENHARIA DE SÃO CARLOS \\ DEPARTAMENTO DE ENGENHARIA ELÉTRICA
}

\title{
SISTEMATIZAÇÃO CRÍTICA DAS TENDÊNCIAS DE PADRONIZAÇÃO DE ARQUITETURAS E PROTOCOLOS EM REDES ÓPTICAS
}

\author{
Eduardo José Aloia
}

Dissertação apresentada à Escola de
Engenharia de São Carlos da
Universidade de São Paulo, como
parte dos requisitos para obtenção do
título de Mestre em Engenharia
Elétrica

Orientador: Prof. Dr. Murilo Araujo Romero

São Carlos, SP 


\section{DEDICATÓRIA}

Dedico esta dissertação aos meus pais Antonio Aloia e Umbelina Martins Aloia. Ao primeiro por proporcionar do plano espiritual onde se encontra, a coragem para a execução deste trabalho. À D. Umbelina pelo amor e a certeza na concretização deste trabalho, certeza esta, às vezes bem maior do que a minha.

Dedico aos meus irmãos Terezinha e Aloi e a meus sobrinhos Fabrício, Gustavo, Isabela e João Victor pelo apoio incondicional.

Dedico também, ao leitor, que se predispuser a embarcar nesta viagem. Encontrará aqui não apenas conceitos sobre redes ópticas, encontrará horas e horas de pesquisa na busca de entendimento e descrição de inúmeros conceitos. Portanto, caro leitor, não queira ler este trabalho de maneira afoita, aprecie as belezas do caminho, e engenhosidade humana atrás de cada conceito aqui apresentado. Esteja certo, prezado viajante, sua leitura será recompensada pela descoberta da grande capacidade humana de ousar. 


\section{AGRADECIMENTOS}

Agradeço ao Professor Dr. Murilo Araujo Romero pelos precisos e valiosos comentários a respeito do caminho a seguir para a concretização deste trabalho. Agradeço ao Professor Dr. Amilcar Careli César pela paciência e observações corretas.

Agradeço também ao Professor Dr. José Carlos Sartori pelas palavras de incentivo e confiança.

Agradeço à funcionária da Secretaria de Pós-Graduação, Marisa, pela responsabilidade e dedicação com que tratou dos trâmites burocráticos deste trabalho.

Felicidade não se busca, se compartilha

PARA SER GRANDE, sê inteiro: nada

Teu exagera ou exclui.

Sê todo em cada coisa. Põe quanto és

No mínimo que fazes.

Assim em cada lago a lua toda

Brilha, porque alta vive.

Fernando Pessoa 





\section{SUMÁRIO}

LISTA DE FIGURAS

\section{CAPÍTULO 1}

INTRODUÇÃO 1

\section{CAPÍTULO 2}

LIMITAÇÕES DE DESEMPENHO DEVIDO À INSERÇÃO DE OVERHEADS DAS PRINCIPAIS ARQUITETURAS MULTICAMADAS

2.1 INTRODUÇÃO 15

2.2 IP SOBRE ATM SOBRE SONET/SDH . 16

2.3 IP SOBRE SONET/SDH. 18

2.4 CÁlCUlO DE OVERHEAD INTRODUZIDO PELAS VÁRIAS PILHAS DE

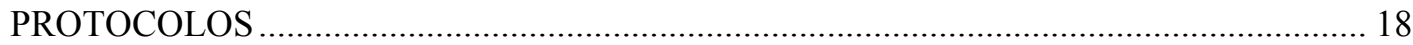

2.4.1 Overhead inserido pela encapsulação SONET/SDH ................................................. 20

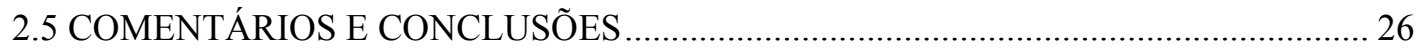

\section{CAPÍTULO 3}

APLICAÇÃO DA ARQUITETURA MPLS NA IMPLEMENTAÇÃO DE ENGENHARIA DE TRÁFEGO EM REDES IP

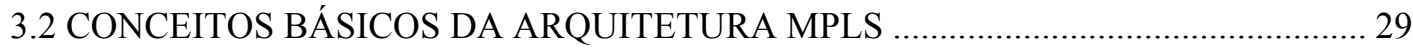

3.2.1 LSRs de borda e LSRs de núcleo ............................................................................. 30

3.2.2 FEC (forward equivalence class)................................................................................. 31

3.2.3 Rótulo (label) e atribuição de rótulo (label binding) .................................................... 31 


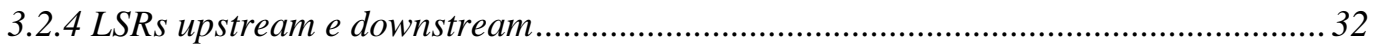

3.2.5 Protocolos de distribuição de rótulos.................................................................................. 33

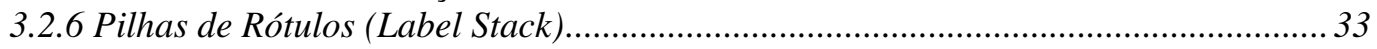

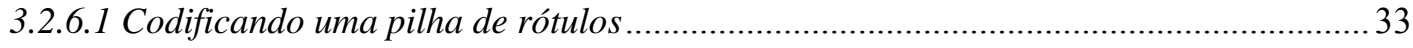

3.2.7 Caminhos Comutados por Rótulos (LSP - Label Switched Paths) .............................. 35

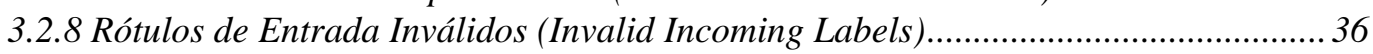

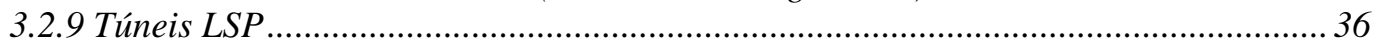

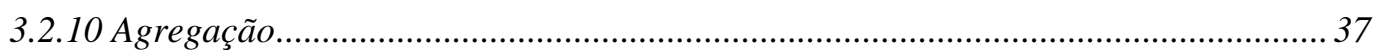

3.2.11 Roteamento Explicito e Hop by hop ......................................................................... 38

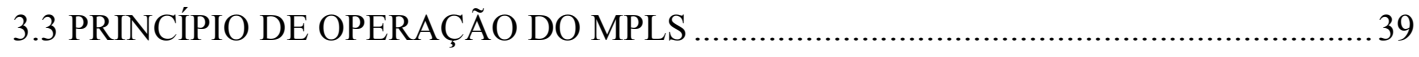

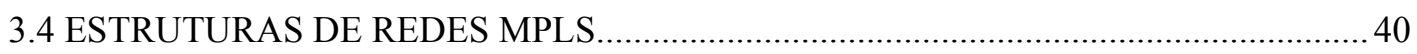

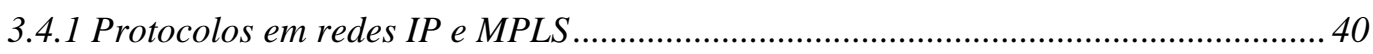

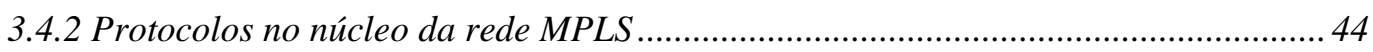

3.4.2.1 Características do Protocolo de distribuição de Rótulos ............................................... 45

3.4.2.2 Características do CR-LDP (constraint-based routing with LDP)............................... 45

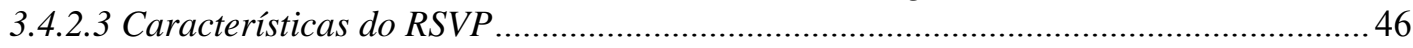

3.4.2.4 Resumo das funções dos Protocolos CR-LDP e RSVP-TE .........................................4 47

3.4.3 Protocolos em dispositivos de borda em redes MPLS................................................. 48

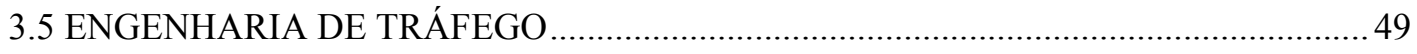

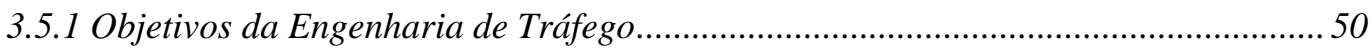

3.5.2 Aplicando o MPLS para Engenharia de Tráfego ...........................................................5

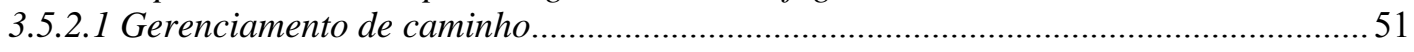

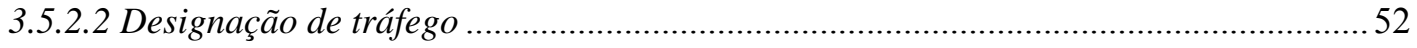

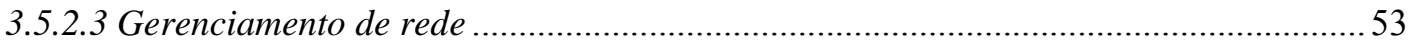

3.5.2.4 Disseminação de informações do estado da rede ..........................................................53

3.5.3 Limitações da Aplicação de MPLS para prover Engenharia de Tráfego .................... 54

CAPÍTULO 4

ARQUITETURA MPLS GENERALIZADA - GENERALIZED MPLS ............................ 55

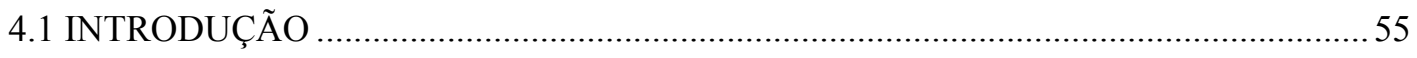

4.2 PROTOCOLO DE GERENCIAMENTO DE ENLACE - LMP ....................................6 60

4.3 EXTENSÕES GMPLS PARA OS PROTOCOLOS DE SINALIZAÇÃO........................61

4.3.1 Formato genérico de requisição de rótulo (Generalized Label Request) .................. 62

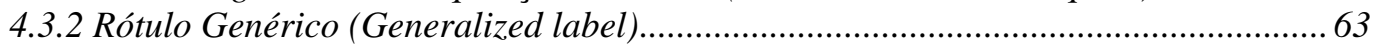

4.3.3 Sugestão de Rótulo (Label suggestion)................................................................ 64

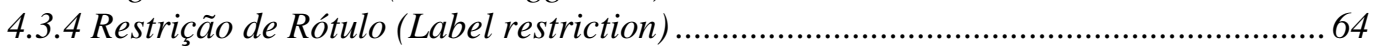

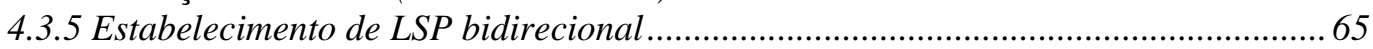

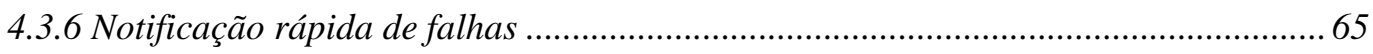

4.3.7 Rotas explícitas com controle de rótulo explícito (explicit label control)...................66

4.3.8 Proteção de enlace ............................................................................................. 66 


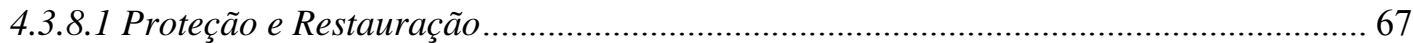

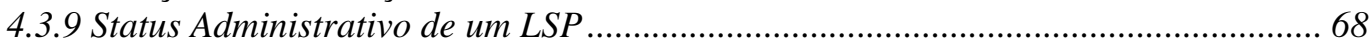

4.4 EXTENSÕES GMPLS PARA OS PROTOCOLOS DE ROTEAMENTO (OSPF E IS-

IS)

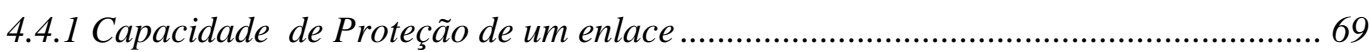

4.4.2 Suporte para enlaces não-numerados (Support for unnumbered links)....................... 70

4.4.3 Descritor da Capacidade de Comutação de uma Interface (Interface Switching

Capability Descriptor) ................................................................................................. 70

4.4.2 Informação de Grupo de Enlaces com Risco Compartilhado (Shared Risk Link Group

Information ) ..................................................................................................................... 72

4.5 EXTENSÕES GMPLS DA ARQUITETURA MPLS....................................................... 72

4.5.1 Maneiras de se implementar as extensões GMPLS...................................................... 74

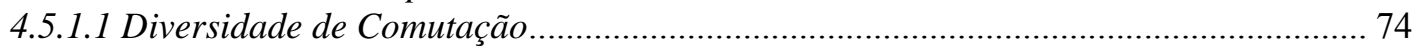

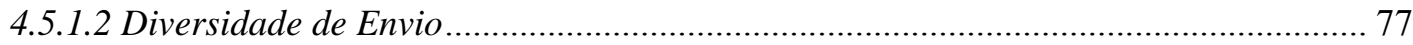

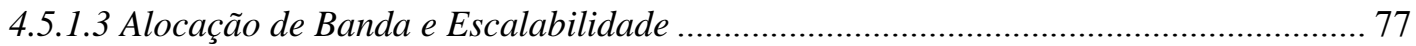

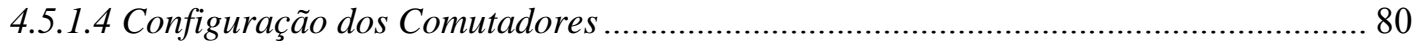

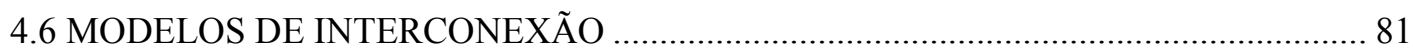

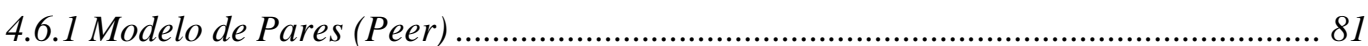

4.6.2 Modelo Coberto (Overlay) ....................................................................................... 82

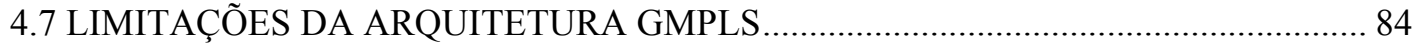

\section{CAPÍTULO 5}

PADRONIZAÇÃO DE REDES ÓPTICAS NO ÂMBITO DO ITU-T E A INSERÇÃO

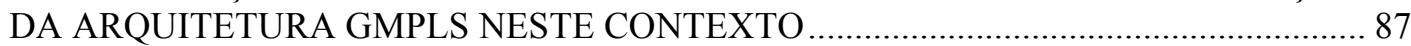

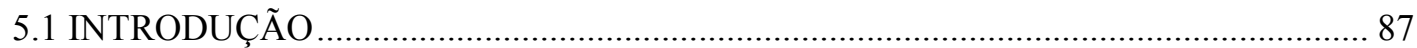

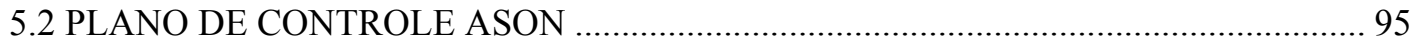

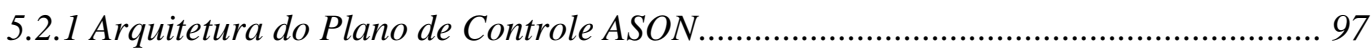

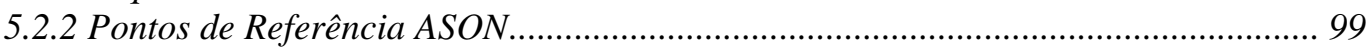

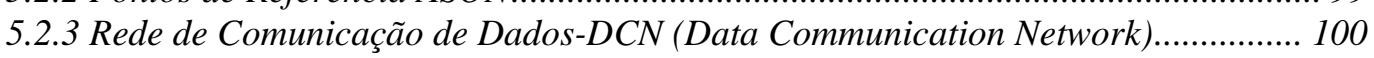

5.3 PROTOCOLOS GMPLS E PONTOS DE REFERÊNCIA ASON …............................... 101

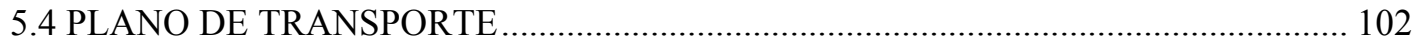

5.4.1 Formato do quadro especificado na Recomendação ITU-T G 709 para a camada

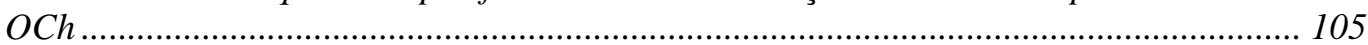

5.4.1.1 Descrição do overhead introduzido pela camada OPU, ODU e OTU........................ 106

5.4.2 Descrição dos overheads introduzidos pelas camadas OCh, OMS e OTS ............... 109

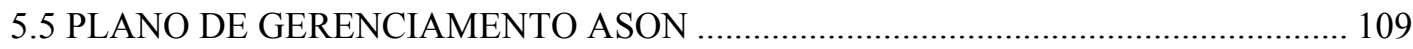

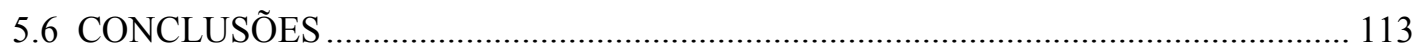




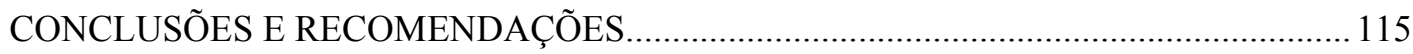

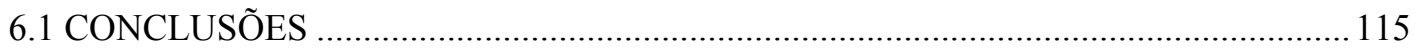

6.2 RECOMENDAÇÕES PARA TRABALHOS FUTUROS ............................................ 117

REFERÊNCIAS BIBLIOGRÁFICAS

APÊNDICE I

I.1 SIGNIFICADO DOS BLOCOS DE UM QUADRO AAL-5 ……............................. 129

I.2 SIGNIFICADO DOS BLOCOS DE UM QUADRO PPP-HDLC ……........................... 130

I.3 DETALHAMENTO DOS CÁLCULOS DE OVERHEAD ............................................... 132

I.3.1 Overhead inserido pela encapsulação IP/ATM .........................................................132

I.3.1.1 Cálculo do overhead médio ponderado para a arquitetura IP/ATM ........................134

I.3.2 Overhead inserido pelo encapsulação IP/PPP/HDLC .......................................... 135

I.3.2.1 Cálculo do overhead médio ponderado para a arquitetura IP/PPP/HDLC ............... 135

\begin{tabular}{lr} 
APÊNDICE II & $\mathbf{1 3 7}$ \\
\hline
\end{tabular}

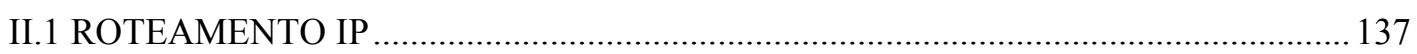

II.2 CONFIGURAÇÃO DE UM LSP UTILIZANDO O PROTOCOLO CR-LDP ............... 139

II.3 CONFIGURAÇÃO DE UM LSP UTILIZANDO O PROTOCOLO RSVP..................... 140

III.1 FUNÇÕES DO LMP (LINK MANAGEMENT PROTOCOL) ...................................... 143

III.1.1 Gerenciamento do canal de controle (control channel management)..................... 143

III.1.2 Correlação da propriedade do link (Link Property Correlation) ............................ 144

III.1.3 Verificação da conectividade do enlace (link connectivity verification)..................146

III.1.4 Gerenciamento de falhas (fault management)..................................................... 147

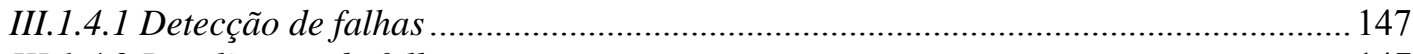

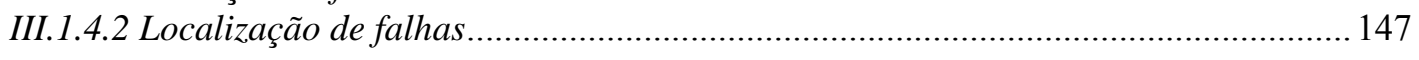

III.2 EXTENSÕES GMPLS PARA OS PROTOCOLOS DE SINALIZAÇÃO...................... 148

III.2.1 Generalized Label Request .................................................................................... 148

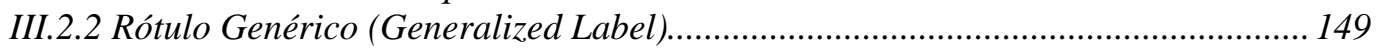

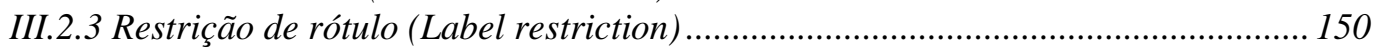

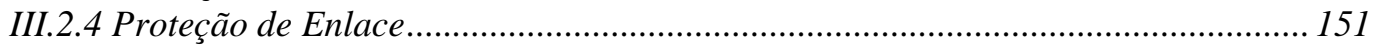


IV.1 DESCRIÇÃO DO OVERHEAD INTRODUZIDO PELA SUBCAMADA OPU ......... 153

IV.2 DESCRIÇÃO DO OVERHEAD INTRODUZIDO PELA SUBCAMADA OTU........ 154

IV.3 DESCRIÇÃO DO OVERHEAD INTRODUZIDO PELA SUBCAMADA ODU ....... 156 


\section{LISTA DE FIGURAS}

FIGURA 1.1: Janelas ópticas do espectro eletromagnético. 3

FIGURA 1.2: Representação esquemática de amplificador óptico EDFA de um estágio. O comprimento de onda bombeado é mostrado em azul e o comprimento de onda do sinal é mostrado em vermelho.

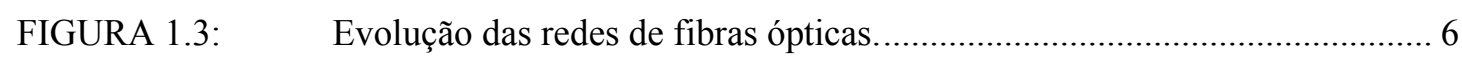

FIGURA 1.4: Exemplo de OADM não reconfigurável. .................................................. 7

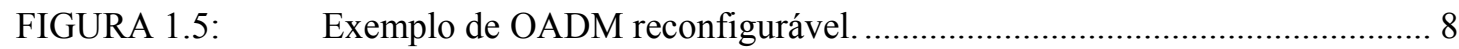

FIGURA 1.6: a) Ilustração esquemática de arquitetura 2D; b) arquitetura 2D NxN

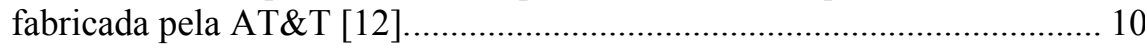

FIGURA 1.7: a) Ilustração de uma arquitetura 3D - 2N; b) Ilustração da reflexão de um feixe de luz usando-se um microespelho com dois eixos de liberdade;

c) Protótipo de um microespelho. 11

FIGURA 1.8: $\quad$ Evolução em direção a arquitetura de redes ópticas com duas camadas.... 13

FIGURA 1.9: a) Redes Ópticas com sub-redes totalmente transparentes conectadas umas às outras através de OEOs e usuários conectados via roteadores; b) Plano de controle comum, visão GMPLS.

FIGURA 2.1: Arquiteturas multicamadas para transporte de IP em redes ópticas. As setas em vermelho apresentam as arquiteturas especificadas neste

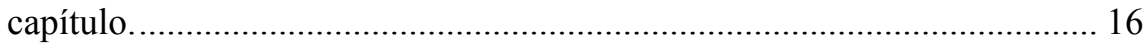

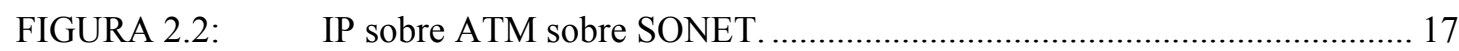

FIGURA 2.3: $\quad$ IP over SONET usando encapsulamento PPP/HDLC.................................... 19

FIGURA 2.4: $\quad$ Subcamadas da camada física do padrão SONET/SDH. ............................. 21

FIGURA 2.5: Quadro básico do padrão SONET............................................................ 22

FIGURA 2.6: Quadro básico do padrão SDH com os quatro tipos de overhead................ 23

FIGURA 2.7: Estrutura simplificada da hierarquia SDH. ................................................. 24

FIGURA 3.1: Formas de transportar um rótulo em um pacote: a) Encapsulação genérica; $\quad$ b) Encapsulação de label da camada 2. …....................... 32

FIGURA 3.2: Rótulo (label) representado por 4 bytes.................................................. 35

FIGURA 3.3: $\quad$ Exemplo de um túnel LSP formado pela seqüência $<\mathrm{R}_{2}, \mathrm{R}_{21}, \mathrm{R}_{22}, \mathrm{R}_{23}$, $\mathrm{R}_{3}>$ (linhas vermelhas), transportando um LSP formado pela seqüência

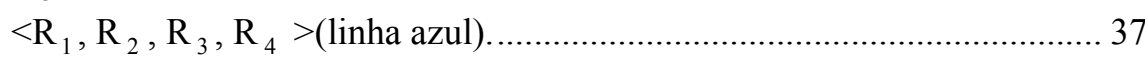

FIGURA 3.4: Fases de configuração de um LSP. O LSR $\mathrm{R}_{1}$ é especificado como o LSR upstream e o LSR $\mathrm{R}_{4}$ como dowstream.............................................. 39

FIGURA 3.5: Arquitetura geral de uma rede MPLS. ......................................................... 41 
FIGURA 3.6: $\quad$ Pilhas simplificadas de Protocolos para IP e MPLS [28]

FIGURA 4.1: Formas de configurar conexões em: a) Redes com gerenciamento centralizado e b) Redes com gerenciamento distribuído. .........................56

FIGURA 4.2: $\quad$ Diagrama de blocos da arquitetura GMPLS ...........................................5

FIGURA 4.3: $\quad$ Extensões GMPLS para os protocolos de sinalização................................62

FIGURA 4.4: a) Em um mecanismo de proteção 1+1 uma conexão é transmitida simultaneamente sobre dois canais (um operando e outro de proteção) e um seletor é usado no nó destino para escolher o melhor sinal. Se uma falha ocorre (1) o nó destino comuta para o enlace de proteção. b) Em um mecanismo de proteção 1:1 um dedicado enlace de reserva é préalocado para o enlace primário. Se uma falha ocorrer (1) o protocolo LMP é usado para localizar a falha. Uma vez a falha tenha sido localizada (2) uma mensagem refresh (RSVP) pode ser usada para indicar o caminho a ser comutado e (3) ambos os nós deveriam comutar

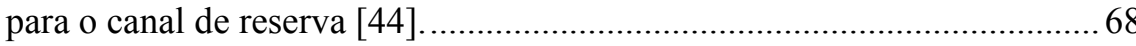

FIGURA 4.5: $\quad$ Extensões GMPLS para os protocolos de roteamento..............................69

FIGURA 4.6: $\quad$ Suporte a enlaces não numerados com os identificadores (32 bits) locais e remotos. ................................................................................ 71

FIGURA 4.7: $\quad$ O LSP1 é configurado de $\mathrm{R}_{0}$ até $\mathrm{R}_{10}$ com a largura de banda de 100 $\mathrm{Mb} / \mathrm{s}$. O LSP1 é agrupado nos LSPs 2, 3 e 4, respectivamente. O roteador $\mathrm{R}_{0}$ mapeia os pacotes para o LSP1. Os roteadores $\mathrm{R}_{1}$ e $\mathrm{R}_{9}$ comutam pacotes. $\mathrm{S}_{2}$ e $\mathrm{S}_{8}$ são comutadores (switches) $\mathrm{SDH}$. Os dispositivos $\mathrm{O}_{3}$ e $\mathrm{O}_{7}$ são comutadores ópticos. Sua função é comutar o enlace STM-4 recebido em forma de um comprimento de onda (lambda) para um sistema DWDM, o qual será comutado pelos $\mathrm{OXCs} \mathrm{P}_{4}$ a $\mathrm{P}_{6}$. O enlace entre $\mathrm{R}_{0}$ e $\mathrm{R}_{1}$ é Fast-Ethernet, entre $\mathrm{R}_{1}$ e $\mathrm{S}_{2}$ um STM-1, entre $\mathrm{S}_{2}$ e $\mathrm{O}_{3}$ um STM-4 especificado em um comprimento de onda, entre $\mathrm{O}_{3}$ e $\mathrm{P}_{4}$ um sistema DWDM com 16 comprimentos de onda, cada um capaz de transportar um STM-4. Tais comprimentos de onda são comutados pelos OXCs. O enlace entre $\mathrm{O}_{7}$ e $\mathrm{S}_{8}$ é um STM-4 recebido em forma de um comprimento de onda, entre $\mathrm{S}_{8}$ e $\mathrm{R}_{9}$ um STM-1 e entre $\mathrm{R}_{9}$ e $\mathrm{R}_{10}$ Fast-Ethernet.

FIGURA 4.8: Uma mensagem PATH Request (path 1) é gerada no roteador $\mathrm{R}_{0} \mathrm{e}$ enviada a $R_{1}$. No roteador $R_{1}$ este pedido dispara uma solicitação para um novo LSP ( $\operatorname{LS~} \mathrm{P}_{2}$ ) de $\mathrm{R}_{1}$ a $\mathrm{R}_{9}$. Esta dinâmica de criação é repetida até a mensagem path 4 ser gerada na switch $\mathrm{O}_{3}$. Seguindo o sucesso na configuração do $\mathrm{LSP}_{4}$ traduzido pelo recebimento da mensagem Resv, o $\mathrm{LSP}_{3}$ é tunelado através do $\mathrm{LSP}_{4}$. Este processo de formação de LSP, na qual um pedido de LSP de baixa ordem sendo canalizado através de um LSP de mais ordem já estabelecido, continua até o LSP inicial (LSP1) ser configurado. 
FIGURA 4.9:

Hierarquia de LSPs .78

FIGURA 4.10: Hierarquia de LSPs, formando os respectivas FA-LSP (forwarding adjacency-LSP), os quais são processadas nas bordas de cada nuvem pertencente a uma mesma tecnologia.

FIGURA 4.11: Modelos de interconexão; a) modelo coberto (overlay), b) modelo de pares (peer).

FIGURA 4.12: Modelo de pares (peer) no qual se constata a utilização de protocolos de sinalização disponíveis na arquitetura GMPLS.

FIGURA 4.13: $\quad$ Modelo coberto (overlay), no qual se constata a utilização de interfaces UNI ( C-Cliente e N-rede padronizados pela OIF) e protocolos de sinalização presentes na arquitetura GMPLS (padronizado pelo IETF). .... 83

FIGURA 5.1: $\quad$ Relacionamento entre os planos da arquitetura ASON [56]. As DCNs são utilizadas com o intuito de formar uma rede de comunicação, onde são trocadas as informações de gerenciamento e ou sinalização entre os diversos planos.

FIGURA 5.2: Recomendações que descrevem o plano de controle ASON. As recomendações em azul serão publicadas no biênio 2003/2004 .................. 96

FIGURA 5.3: Componentes da arquitetura do Plano de Controle ASON ........................... 97

FIGURA 5.4: Interação entre os controladores da parte chamada/chamante e o controlador de chamada de rede................................................................. 98

FIGURA 5.5: Controladores CC e LRM utilizando um controlador de protocolo PC...... 99

FIGURA 5.6: $\quad$ Rede ASON mostrando a divisão de domínios e os pontos de referências UNI, E-NNI e I-NNI. ........................................................................ 100

FIGURA 5.7: a) Subcamadas do plano de transporte; b) Camadas do Plano de

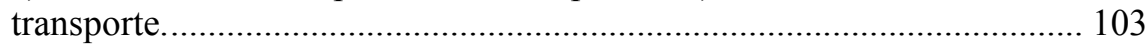

FIGURA 5.8: Camadas do plano de transporte em um enlace de uma rede óptica. ........ 104

FIGURA 5.9: a) Estrutura de quadro utilizada em um canal óptico; b) Detalhe do overhead introduzido pelas camadas OPU, ODU e OTU.......................... 105

FIGURA 5.10: Monitoramento do canal óptico através de múltiplas redes utilizando-se

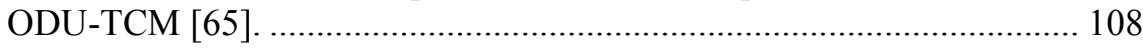

FIGURA 5.11: Modelo organizacional de gerenciamento de uma rede ASON. Terminais LCT (Local Craft Terminal) são para acesso aos objetos gerenciados.... 110

FIGURA 5.12: $\quad$ Funções de gerenciamento do equipamento óptico.................................... 112

FIGURA I.1: Quadro AAL-5, utilizado no encapsulamento IP/ATM............................. 129

FIGURA I.2: $\quad$ Significado dos 8 bytes do Frame (AAL5-CPCS-PDU) ........................... 130

FIGURA I.3: Quadro PPP-HDLC, utilizado no encapsulamento IP/SONET-SDH. ....... 131

FIGURA I.4: Bloco de funções executados no mapeamento

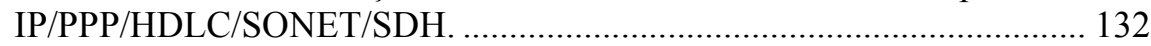

FIGURA II.1: $\quad$ Interconexão de duas redes Ethernet por um backbone IP......................... 138 
FIGURA II.2: $\quad$ Configuração de um LSP utilizando CR-LDP com a mensagem de pedido de rótulo label request e a respectiva resposta label mapping. ...... 139

FIGURA II.3: $\quad$ Configuração de um LSP utilizando RSVP-TE com a mensagem de pedido de rótulo Path Request e a respectiva resposta Resv.

FIGURA III.1.1: Mapeamento de enlaces individuais em um enlace lógico TE.

FIGURA III.3.2: $\quad$ Processo de verificação de link e respectivo canal de controle. 147

FIGURA III.3.3: Informação transportada em um generalized label request. 148

FIGURA III.3.4: Formato de um variável generalized label.

FIGURA III.3.5: Formato de como são transportadas as informações de um conjunto de rótulos (label set).

FIGURA III.3.6: Formato da função Informação de proteção (protection information), transportada pelos protocolos de sinalização.

FIGURA IV.1: a) Overhead introduzido pela camada OPU com o respectivo subcampo PSI; $\quad$ b) Mapeamento para sinais CBR (constant bit rate) com taxas de $2.5,10$ e 40 GBPS.

FIGURA IV.2: a) Overhead introduzido pela camada OTU; b) Subcampos do campo SM.

FIGURA IV 3: $\quad$ Configuração do subcampo BIP-8.................................................. 156

FIGURA IV 4: $\quad$ Overhead introduzido pela camada ODU com os respectivos subcampos PM e TCM. 


\section{LISTA DE TABELAS}

TABELA 2.1: Distribuição de tamanhos de pacotes no backbone Internet da MCI

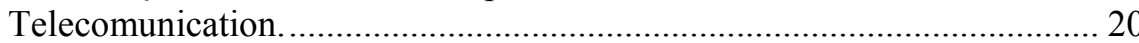

TABELA 2.2: $\quad$ Porcentagem de overhead de PPP/HDLC e ATM [20] ............................... 20

TABELA 2.3: Taxas de multiplexação e overhead da hierarquia SONET e SDH............. 25

TABELA 5.1: $\quad$ Recomendações que especificam a rede de transporte óptica (ASON) padronizada pelo ITU-T. As recomendações escritas na cor preta especificam a arquitetura da rede, na cor azul definem o plano de controle, na cor verde estabelecem o plano de gerenciamento e na cor vermelha implementam o plano de transporte. ............................................ 90

TABELA 5.2: $\quad$ Tempo de transmissão do quadro formado pela camada OCh [56].......... 106 


\section{LISTA DE ABREVIATURAS E SIGLAS}

\begin{tabular}{|c|c|}
\hline AAL-5 & -Adaptation Layer Type 5 \\
\hline AS & -Autonomous System \\
\hline ASON & -Automatically Switched Optical Networks \\
\hline ATM & - Asynchronous Transfer Mode \\
\hline AU & - Administrative Unit - Unidade Administrativa \\
\hline AUG & - Administrative Unit Group - Grupo de Unidades Administrativas \\
\hline BGP & - Border Gateway Protocol \\
\hline CR-LDP & - Constraint-based Routing with Label Distribution Protocol \\
\hline DFB & - Distribute Feedback Lasers \\
\hline DSF & - Dispersion-Shifted Fibers \\
\hline DWDM & - Dense Wavelength Division Multiplexing \\
\hline $\mathrm{DXC}$ & - Digital Cross-Conects \\
\hline EDFA & - Erbium Doped Fiber Amplifier \\
\hline FDM & - Frequency Division Multiplexing \\
\hline FEC & - forward equivalence class \\
\hline FSC & - Fiber-Switch Capable \\
\hline GMPLS & - Generalized Multiprotocol Label Switching \\
\hline HDLC & - High-level Data Link Control \\
\hline IETF & - Internet Engineering Task Force \\
\hline I-NNI & - Interior Node to Node Interface \\
\hline IP & - Internet Protocol \\
\hline IS-IS & - Interior System to Interior System \\
\hline ISP & - Internet Service Provider \\
\hline ITU & - International Telecommunications Union \\
\hline LDP & - Label Distribution Protocol \\
\hline LLC & - Logical link control \\
\hline LMP & - Link Management Protocol \\
\hline LSC & - Lambda Switch Capable \\
\hline LSP & - Label Switched Paths \\
\hline LSR & - Label Switch Routers \\
\hline MEMS & - Micro-Electromechanical Systems \\
\hline MPLS & - Multiprotocol Label Switching \\
\hline NNI & - Network Node Interface \\
\hline NZDF & - Nonzero-Disperson Fibers \\
\hline OADM & - Optical Add/Drop Multiplexer \\
\hline $\mathrm{OC}$ & - Optical Carrier \\
\hline $\mathrm{OCH}$ & - Optical Channel \\
\hline ODU & - Optical Channel Data Unit \\
\hline $\mathrm{OEO}$ & - Óptico-Eletrônico-Óptico \\
\hline OIF & - Optical Internetworking Fórum \\
\hline OMS & - Optical Multiplex Section \\
\hline OPU & - Optical Channel Payload Unit \\
\hline OSPF & - Open Shortest Path First \\
\hline
\end{tabular}




$\begin{array}{ll}\text { OTS } & \text { - Optical Transmission Section } \\ \text { OTU } & \text { - Optical Transport Unit } \\ \text { OXC } & \text { - Optical Cross Connect } \\ \text { PCM } & \text { - Pulse Code Modulation } \\ \text { PPP } & \text { - Point-to-Point Protocol } \\ \text { PSC } & \text { - Packet Switch Capable } \\ \text { QoS } & \text { - Quality of Service } \\ \text { RFC } & \text { - Request For Comment } \\ \text { RIP } & \text { - Routing Information Protocol } \\ \text { RSVP } & \text { - Resource Reservation Protocol } \\ \text { SDH } & \text { - Synchronous Digital Hierarchy } \\ \text { SONET } & \text { - Synchronous Optical Network } \\ \text { SPE } & \text { - Synchronous Payload Envelope } \\ \text { STM } & \text { - Módulo de Transporte Síncrono } \\ \text { STS } & \text { - Synchronous Transport Signal } \\ \text { TCP } & \text { - Transport Control Protocol } \\ \text { TDM } & \text { - Time Division Multiplexing } \\ \text { TE } & \text { - Traffic Enginneering } \\ \text { TU } & \text { - Unidade Tributária } \\ \text { TUG } & \text { - Grupos de Unidades Tributárias } \\ \text { UDP } & \text { - Universal Datagram Protocol } \\ \text { UNI } & \text { - User Network Interface } \\ \text { VC } & \text { - Virtual Container } \\ \text { VPN } & \text { - Virtual Private Network } \\ \text { WDM } & \text { - Wavelength Division Multiplexing }\end{array}$




\section{RESUMO}

O principal objetivo deste trabalho é analisar a arquitetura das redes ópticas, discutir o estado da arte dos protocolos necessários ao seu desenvolvimento, e avaliar os vários esforços empregados para a padronização destas por diferentes instituições. Conceitualmente, redes ópticas são aquelas nas quais a tecnologia dominante na camada física é a fibra óptica. Tais redes originaram-se no início dos anos $80 \mathrm{com}$ a utilização dos cabos de fibras ópticas monomodo, tendo evoluído com a introdução de uma nova base tecnológica composta por novos tipos de fibras ópticas, amplificadores ópticos, sistemas DWDM e componentes ópticos, como multiplexadores e demultiplexadores "add/drop" e "cross-connects" ópticos. O desenvolvimento destes componentes possibilitará a evolução dos simples enlaces DWDM ponto a ponto para a rede totalmente óptica. Neste trabalho é analisado como as aplicações interagirão com esta camada física. Tal interação tem provado ser um desafio dentro da atual arquitetura das redes de dados, no intuito de disponibilizar soluções que habilitem o transporte de um grande volume de tráfego de forma eficiente. Neste sentido é analisado o emprego de tecnologias como o MPLS (Multiprotocol Label Switching) e sua evolução, o Generalized MPLS (GMPLS). Finalmente apresenta-se a versão do ITU para arquitetura de redes ópticas, e a conseqüente inserção do GMPLS nesta padronização.

Palavras-chaves: Redes Ópticas, MPLS, GMPLS 


\begin{abstract}
The main objective of this work is to analyze the evolution trends for the architecture of the optical networks, discuss the state of the art protocols required for its development and evaluate the various efforts being carried out by different institutions in order to achieve standardization. In this context, optical networks are those in which the dominant technology in the physical layer is the optical fiber. Such networks arose in the early eighties with the use of the monomodo fiber optic cables and were further developed with the introduction of a new technological base composed by new types optical fibers, optical amplifiers, DWDM systems and optical components such as add/drop multiplexers and optical cross-connects. The introduction of these components will make possible the evolution from the simple point-to-point DWDM connections to the all optical networks. In this work we analyze how the applications will interact with this physical layer. Such interaction has been proving to be a challenge within the framework for the current architecture of data networks, aiming at offering solutions to enable the transport of a great volume of traffic in an efficient manner. With this goal in mind, the use of technologies such as MPLS (Multiprotocol Label Switching) and its evolution, the Generalized MPLS (GMPLS) is discussed. Finally, we present the ITU version for the architecture optical networks, addressing its merging with the GMPLS.
\end{abstract}

Keywords: Optical Networks, MPLS, GMPLS 
XXIII 


\section{CAPÍTULO 1}

\section{INTRODUÇÃO}

A utilização dos comprimentos de onda do espectro eletromagnético, do qual a luz visível é apenas uma fração, reveste-se de grande importância, principalmente no aspecto relacionado às comunicações. Utilizam-se faixas deste espectro para efetuar comunicações em meios e formatos diferentes, evoluindo sempre na procura por formas mais rápidas, confiáveis e eficientes, em termos de custo, de propagar a informação. Neste cenário surgem as redes ópticas, nas quais a tecnologia dominante na camada física é a fibra óptica. Tais redes originaram-se no início dos anos 80 com a utilização dos cabos de fibras ópticas monomodo, principalmente nos Estados Unidos, Europa e Japão. O emprego desta nova tecnologia gerou uma demanda por padrões ópticos, devido à necessidade das várias concessionárias locais, cada uma com seu próprio sistema de fibras ópticas, de se conectarem às concessionárias de longa distância, particularmente após a divisão da AT\&T nos Estados Unidos em 1984 [1]. Surge então o padrão Synchronous Optical Network/Synchronous Digital Hierarchy (SONET - Estados Unidos/SDH - Padrão Internacional), padronizado pelo ITU-T (International Telecommunications Union, Genebra, Suiça), com o intuito, segundo TANENBAUM [2], de cumprir algumas premissas: possibilidade de interconectar diferentes concessionárias em rede, exigindo-se assim um padrão de sinalização comum, com respeito ao sincronismo, à estrutura de enquadramento e outros aspectos; apresentar meios de unificar os sistemas digitais dos Estados Unidos, Europa e Japão todos baseados em canais PCM de $64 \mathrm{kbps}$, mas com diferentes formas de composição de tributários; oferecer um mecanismo para multiplexar vários canais digitais com diferentes taxas de bits e possibilitar recursos de operação, administração e manutenção. Estava criado, portanto, um sistema utilizando a multiplexação por divisão de tempo (Time Division MultiplexingTDM), com a largura de banda da fibra dedicada somente a um canal, alocando segmentos de tempo para os diversos subcanais, configurando um sistema síncrono, controlado por um relógio mestre. 
Esta padronização permitiu uma grande interoperabilidade entre os sistemas SONET/SDH comercializados por diferentes fornecedores e sua conseqüente disseminação, suprindo assim a necessidade de conexão para o serviço de comunicação de voz.

Com o crescimento vertiginoso das aplicações utilizando o protocolo IP (Internet Protocol), a substituição do modelo baseado em tráfego de voz pelo de tráfego de dados tornou-se inevitável. Ditada pela necessidade de maior largura de banda, utilizada para o tráfego de dados gerados pela Internet e suas aplicações correlatas (Virtual Private Network - VPN, aplicações de voz, vídeo e multimídia), e do rápido crescimento do número de usuários, as redes ópticas passaram a incorporar a multiplexação por comprimento de onda (Wavelength Division Multiplexing WDM). Tal mudança procurou atender aos consumidores, pois estes passaram a exigir maior largura de banda para suas aplicações de tráfego de dados, e aos provedores de serviços, pois estes necessitavam aumentar a capacidade de utilização das fibras já instaladas.

Similar aos antigos enlaces de dados, os quais utilizavam a multiplexação por divisão de freqüência (Frequency Division Multiplexing - FDM), na qual a multiplexação de canais era realizada alocando-se faixas de freqüência para cada canal e transmitindo-os em um único meio de transmissão, a multiplexação por comprimento de onda é o processo no qual cada canal é associado a um comprimento de onda específico e transmitido via fibras ópticas.

Os sistemas WDM surgiram na década de 80 e possuíam inicialmente a capacidade de multiplexar dois canais de diferentes e largamente espaçados comprimentos de onda (1310 nm e $1550 \mathrm{~nm})$. No último terço da década de 90 estes sistemas já haviam evoluído para a densa multiplexação de comprimentos de onda (Dense Wavelength Division Multiplexing - DWDM) apresentando tipicamente 16 canais com os respectivos comprimentos de onda, ampliados para 30 canais no final da década. Atualmente sistemas DWDM com 80 canais estão sendo testados [3].

No projeto de sistemas DWDM deve-se levar em conta o espaçamento entre canais. Desta maneira, para garantir uma total interoperabilidade de multiplexadores, demultiplexadores e demais componentes, o ITU definiu em 2002 a grade de comprimento de onda para tais sistemas, por meio da recomendação ITU-T G 694.1 "Spectral grids for WDM applications: DWDM frequency grid”, padronizando o espaçamento entre canais. A grade de freqüências padronizadas está centrada na freqüência de 193,1 THz (1552,52 nm) e suporta espaçamento entre canais de 12,5, 25, 50 e $100 \mathrm{GHz}$. Como exemplo, em uma fibra com espaçamento entre canais de $100 \mathrm{GHz}$ as freqüências permitidas são definidas por $193,1+\mathrm{n} \times 0,1$, onde $\mathrm{n}$ é um número inteiro positivo ou negativo, incluindo o 0 . No espaçamento de canal de $100 \mathrm{GHz}$ cada 
canal transporta um sinal de 10 Gbps. A utilização de menor espaçamento entre canais dependerá do desenvolvimento tecnológico de dispositivos ópticos como, por exemplo, lasers e filtros. Ressalta-se que, a fim de suprir a demanda resultante por comprimentos de onda bem definidos, os lasers semicondutores denominados distributed feedback lasers (DFBs) [4], por possuírem largura espectral mais estreita, apresentam-se como a melhor opção em relação aos lasers Fabry-Perot, sendo utilizados como os elementos geradores dos comprimentos de onda padronizados pelo ITU.

Esta padronização utiliza uma das regiões do espectro eletromagnético na qual a atenuação óptica na sílica torna-se menor. Estas regiões localizam-se entre regiões de grande absorção e são chamadas de "janelas", sendo as mais importantes listadas na Figura 1.1. Os primeiros sistemas ópticos utilizavam a primeira janela (centrada em $850 \mathrm{~nm})$. A segunda janela $(1310 \mathrm{~nm})$ logo provou ser superior em virtude de sua menor atenuação, seguida da terceira janela, centrada em $1550 \mathrm{~nm}$ (Banda C), na qual a perda óptica torna-se ainda menor. Hoje a quarta janela (Banda L) próxima de $1600 \mathrm{~nm}$, canaliza o desenvolvimento da tecnologia óptica [5].

\section{Ultravioleta Luz Visível Infravermelho}

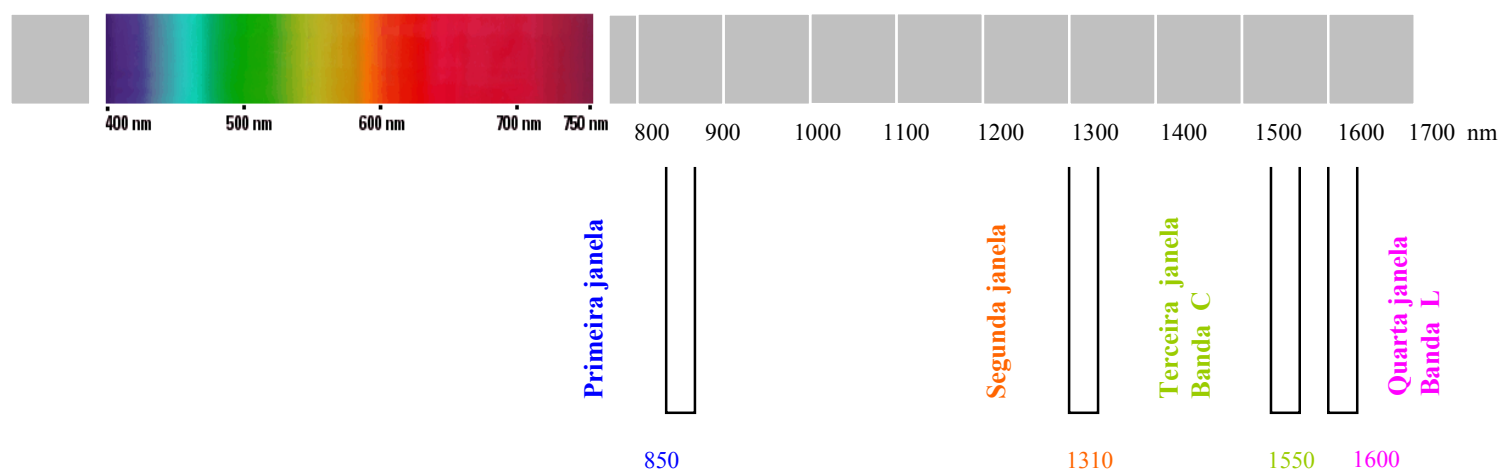

FIGURA 1.1: Janelas ópticas do espectro eletromagnético.

Ainda que se tente reduzi-la, trabalhando em comprimentos de onda nos quais a atenuação apresente-se menor, esta existe e deve ser compensada, utilizando-se amplificadores. Os amplificadores utilizando fibras dopadas com érbio (erbium doped fiber amplifier - EDFA), surgidos no final da década de 80 (Figura 1.2), estendem os limites dos enlaces DWDM, por prover amplificação do sinal óptico, sem necessidade de convertê-lo para sinal elétrico, sendo transparentes para quaisquer taxas, formatos e sintaxe dos bits, tornando o sistema fotônico menos complexo e, portanto, economicamente mais viável. Apresentam-se assim como resultado 
de intensas pesquisas, com o intuito de disponibilizar um ganho plano para a faixa de comprimentos de onda de interesse [6], estando disponibilizados na banda C (1530 a $1565 \mathrm{~nm})$, e mais recentemente na banda L (1570 a $1620 \mathrm{~nm}$ ).

$\mathrm{Na}$ construção de um amplificador empregando fibra óptica dopada o elemento dopante mais utilizado é o érbio, o qual, quando estimulado por um laser de bombeio com comprimento de onda fixo (980 ou $1480 \mathrm{~nm}$ ), tem seus íons excitados a um nível de energia mais alto, liberando esta energia quando os referidos íons decaem para o seu nível de energia de origem e fótons são emitidos por emissão estimulada. Os EDFAs apresentam ganho de aproximadamente $30 \mathrm{~dB}$. Outros parâmetros essenciais na escolha de um amplificador deste tipo são relação sinal ruído e ganho plano. De fato, o ganho deveria ser plano para prover uma igual amplificação dos vários comprimentos de onda. Todavia, este tipo de amplificador apresenta ganho inerentemente dependente do comprimento de onda. Sendo assim, filtros são usualmente empregados nos amplificadores mais modernos para realizar a desejada correção. Por outro lado, o nível de ruído injetado pelo amplificador deveria ser o menor possível, pois este é cumulativo, sendo, por isto, um fator limitante no número de amplificadores que podem ser concatenados e, por conseqüência, na extensão do enlace óptico. Na prática, a distância entre amplificadores aproxima-se dos $120 \mathrm{~km}$. 


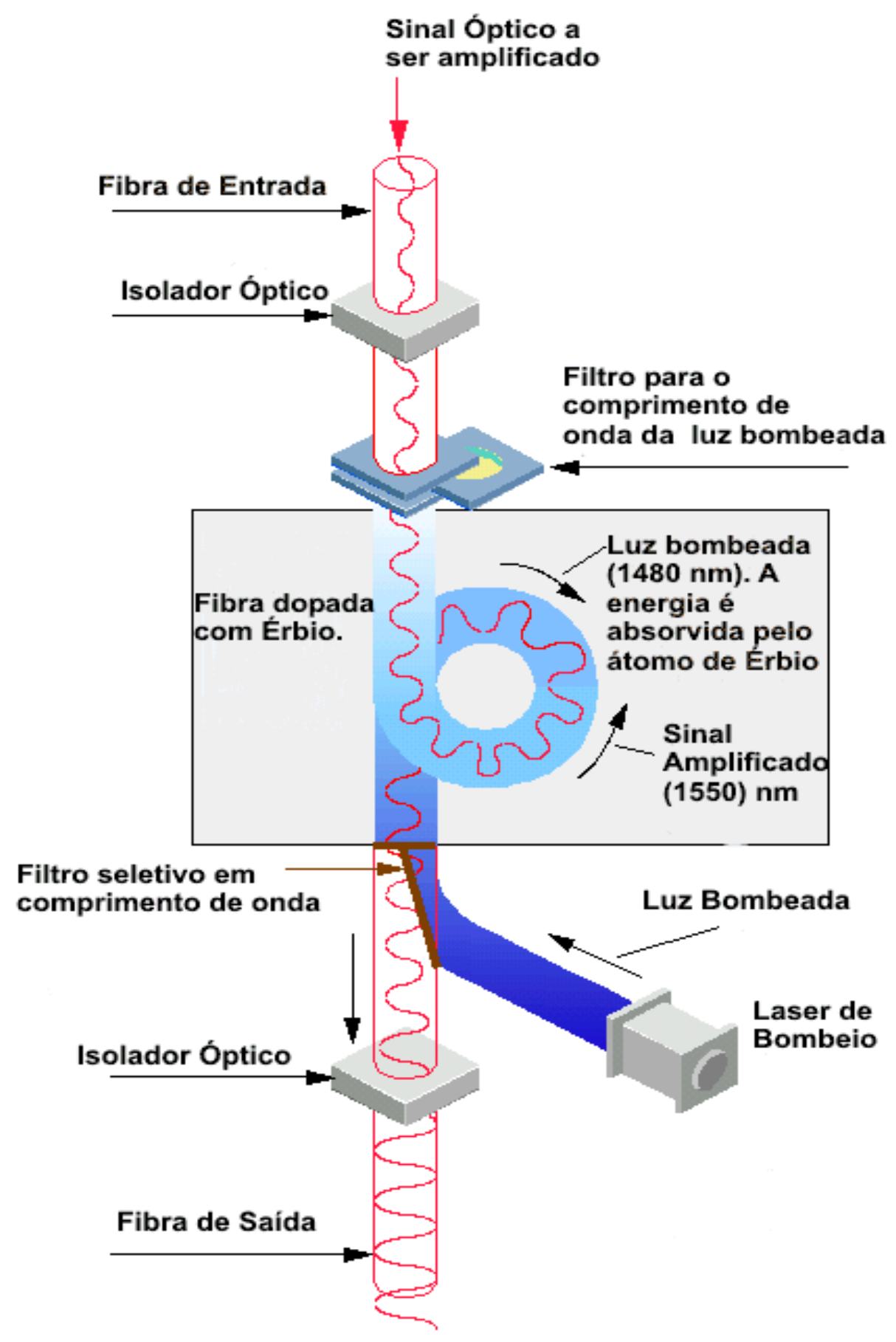

FIGURA 1.2: Representação esquemática de amplificador óptico EDFA de um estágio. O comprimento de onda bombeado é mostrado em azul e o comprimento de onda do sinal é mostrado em vermelho. 
Outros dispositivos ópticos tais como o multiplexador óptico add/drop (optical add/drop multiplexer - OADM) e o optical cross connect (OXC), são cruciais para a implementação das redes totalmente ópticas, sendo apresentados na Figura 1.3. Dispositivos OADM têm como operação básica retirar e inserir comprimentos de onda em cada nó óptico, sendo sua configuração fixa ou reconfigurável. Já os OXCs têm a função de comutar comprimentos de onda entre diferentes fibras, sendo na verdade uma "matriz de comutação".

\section{Tempo}

enlace simples

ponto a ponto

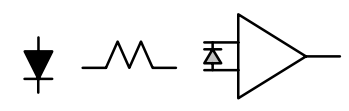

Transmissão WDM com múltiplos enlaces amplificados

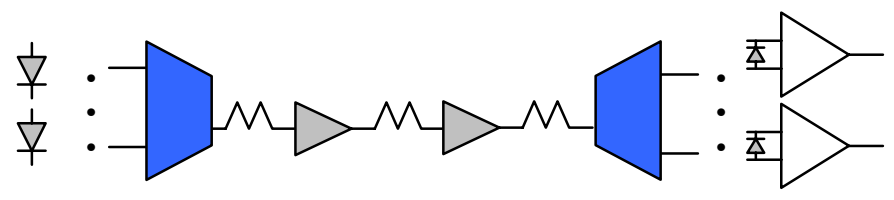

Transmissão WDM com múltiplos enlaces amplificados $e$

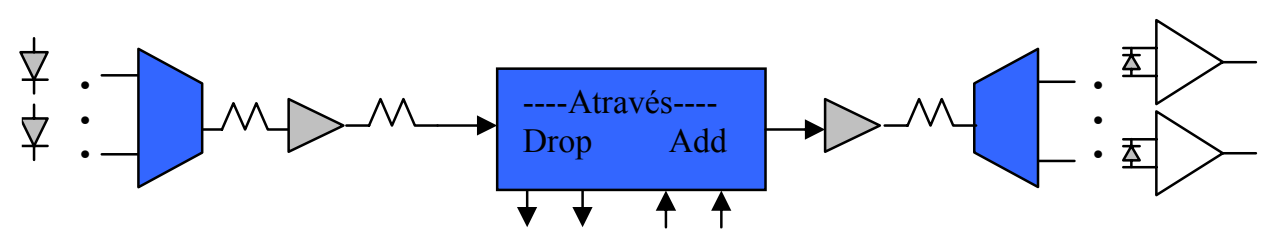
dispositivo fotônico OADM.

Redes totalmente ópticas com dispositivos fotônicos OADMs e OXCs .

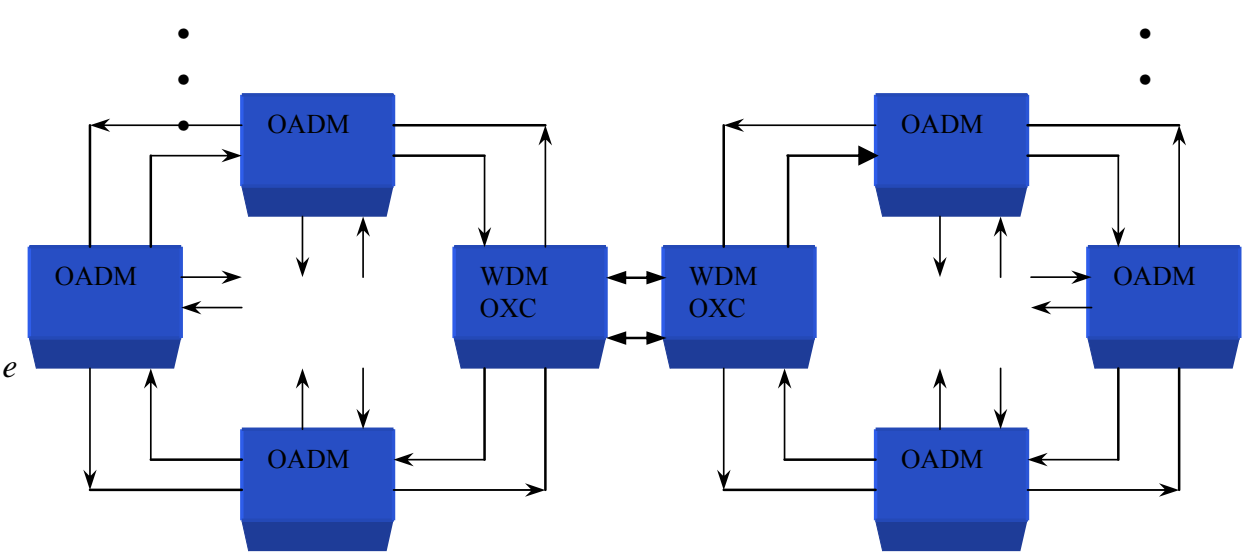

FIGURA 1.3: Evolução das redes de fibras ópticas. 
De um simples enlace ponto a ponto com um único comprimento de onda, típico dos anos 80 [7], passamos a redes ópticas onde o comprimento do enlace estende-se a centenas de quilômetros, empregando amplificadores ópticos EDFAs e multiplexação WDM. A introdução dos EDFAs e de dispositivos fotônicos como os OADMs e OXCs procura evitar a conversão optoeletrônica do sinal em pontos intermediários da rede óptica, minimizando atrasos e buscando aproveitar melhor a grande largura de banda da fibra óptica. Tal fato permitirá a passagem das redes ópticas opacas, nas quais existem nós intermediários onde ocorrem conversões optoeletrônicas, sendo exemplos os sistemas SONET/SDH com comprimento de onda único e sistemas WDM em operação comercial, para as redes ópticas transparentes. De acordo com o cenário descrito no último estágio da Figura 1.4, a retirada e inserção de diferentes comprimentos de onda será implementada por OADMs reconfiguráveis e a interconexão de redes realizar-se-á através de OXCs.

Um OADM não-reconfigurável pode ser construído com a utilização de um circulador óptico e uma rede de Bragg em fibra óptica (Figura 1.4), sendo esta uma pequena seção de fibra modificada para apresentar mudanças periódicas no índice de refração na direção axial. Dependendo do período da rede, uma certa freqüência do sinal incidente (o comprimento de onda de Bragg) é refletido de volta, enquanto outros comprimentos de onda passam sem reflexão [8]. Em outras palavras, a rede de Bragg em fibra óptica atua como um filtro óptico reflexivo pela existência de uma banda de rejeição, a região de freqüência na qual a maior parte da luz incidente é refletida de volta. A banda de rejeição é centrada no comprimento de onda de Bragg $\lambda \mathrm{B}=2 \cdot \bar{n} . \Lambda$, onde $\Lambda$ é o período da rede e $\bar{n}$ é o índice modal efetivo [4].

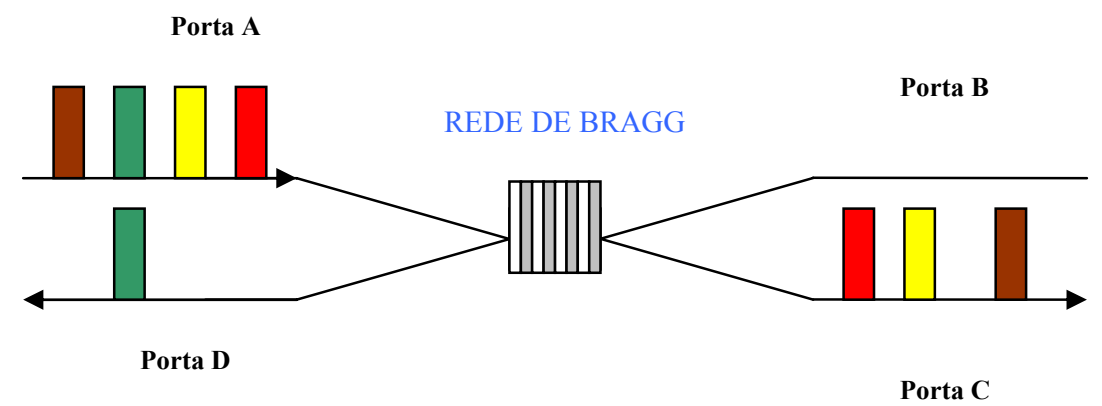

FIGURA 1.4: Exemplo de OADM não reconfigurável.

Por outro lado, um OADM reconfigurável pode retirar e inserir comprimentos de onda dinamicamente, através de operação manual ou via programação, de acordo com o gerenciamento de rede, oferecendo maior flexibilidade para a utilização de canais. Na Figura 1.5 
é apresentada a estrutura esquemática geral de um OADM reconfigurável em óptica integrada. O diagrama de blocos mostra as operações básicas de um multiplexador óptico add/drop programável.

Cada um dos canais é associado a um comprimento de onda específico. Estes canais são inseridos em um divisor óptico onde são direcionados para as $\mathrm{N}$ portas de saída. Em cada uma das portas de saída do acoplador óptico há filtros ópticos fixos que realizam a seleção dos canais de interesse, ou seja, somente o canal desejado passa pelo filtro. Após esta operação o canal é direcionado a uma chave óptica que permite que o mesmo seja retirado (operação "drop") neste ponto ou prossiga no enlace até outro nó da rede. A chave óptica possibilita também a inserção de novos canais (operação "add") [9]. Na saída da chave óptica pode ser colocado um dispositivo conversor de comprimento de onda. Este dispositivo é utilizado para evitar que dois canais com mesmo comprimento de onda sejam eventualmente alocados para o mesmo enlace. Isto provocaria um conflito entre eles, aumentando a possibilidade de bloqueio da transmissão. Após o estágio de conversão é finalmente colocado um combinador óptico. A grande desvantagem deste tipo de multiplexador é a perda de inserção alta devido à divisão de potência, limitando assim o número de portas. Desta maneira, a tendência dominante é utilizar os sistemas microeletromecânicos (Micro-Electromechanical Systems - MEMS) como a principal tecnologia a ser empregada na implementação de OADMs.

\section{Multiplexador Add/Drop}

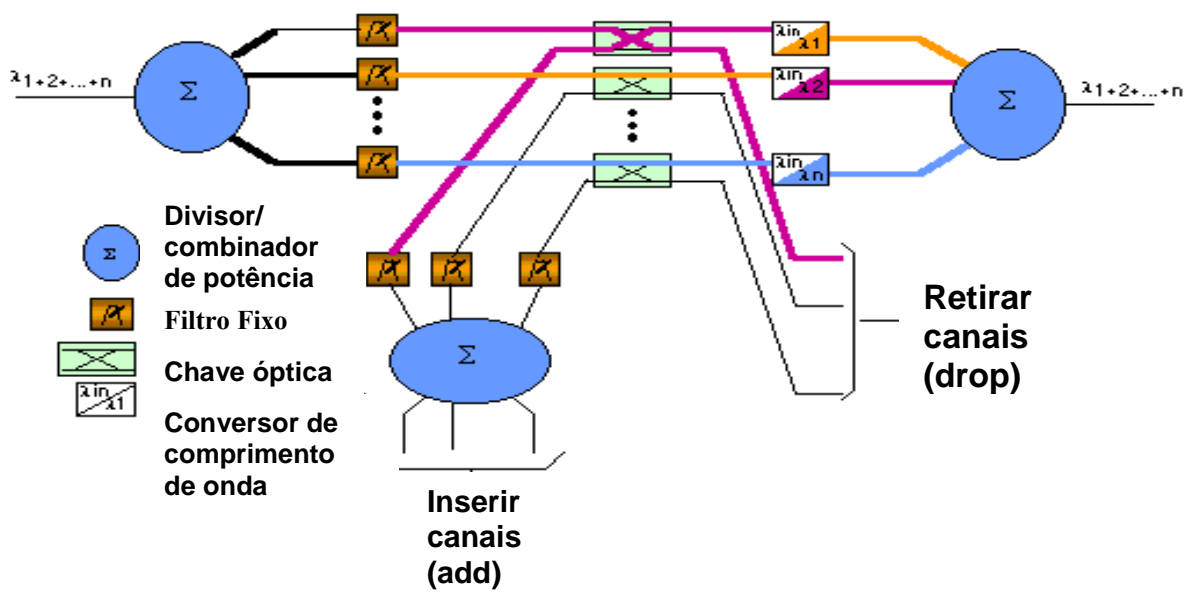

FIGURA 1.5: Exemplo de OADM reconfigurável. 
Em relação ao comutador óptico ou OXC (optical cross-connect), o estado da arte apresenta comutadores optoeletrônicos (óptico-eletrônico-óptico - OEOs) com capacidade para comutar centenas de portas, cada uma suportando taxas de $155 \mathrm{Mb} / \mathrm{s}$ a 2,5 Gb/s. Existem equipamentos comerciais apresentando 512 portas OC-48 $(2,5 \mathrm{~Gb} / \mathrm{s})$ de entrada e 512 portas OC-48 de saída perfazendo uma capacidade agregada de $1,28 \mathrm{~Tb} / \mathrm{s}$ [10]. Por outro lado, a próxima geração de OXCs operará no domínio puramente óptico aumentando a quantidade de portas a milhares, suportando os mais modernos sistemas DWDM. Além de proporcionarem proteção, restauração, roteamento de comprimento de onda e monitoramento de desempenho, estes dispositivos serão estritamente "nonblocking", ou seja, qualquer entrada poderá ser comutada para qualquer saída, não afetando as conexões já estabelecidas.

Os sistemas MEMS são componentes semicondutores que utilizam dispositivos mecânicos fabricados a partir de materiais com excelentes propriedades mecânicas e elétricas ( $\mathrm{Si}$ e $\mathrm{SIO}_{2}$, por exemplo). Nestes sistemas, microespelhos refletem o sinal de entrada para uma porta de saída, sendo a força de atuação requerida para mover tal dispositivo originada em forças eletrostáticas ou magnéticas. Podemos distinguir duas abordagens dos sistemas MEMS para comutação óptica: 2D (digital) ou 3D (analógico).

Em comutadores 2D (Figura 1.6) são necessários $\mathrm{N}^{2}$ microespelhos, sendo $\mathrm{N}$ o número de portas a serem comutadas. Em tal sistema, a forma de movimentação dos microespelhos é digital pois a posição deles é biestável (ligado "on” ou desligado "off”). Sendo ativados, os microespelhos movem-se em direção ao caminho do feixe de luz, refletindo-o para uma porta de saída em uma direção perpendicular à original (estado ligado). Caso contrário, os microespelhos permanecerão imóveis e o referido feixe será transmitido (estado desligado). Devido a perdas ópticas originadas pela disposição dos feixes ópticos em um mesmo plano, ou seja, às diferentes distâncias percorridas por um feixe de luz, a ocorrência de perdas devido à propagação da luz não é uniforme para todas as portas. A diferença entre a perda mínima e máxima de um sistema 2D de 16x16 (256 microespelhos) apresenta um valor maior do que $5 \mathrm{~dB}$ [11]. Por isso comutadores $2 \mathrm{D}$ encontram aplicações somente em sistemas que necessitem comutar pequeno número de portas.

A arquitetura 3D (Figura 1.7), fazendo uso de uma dimensão espacial não-planar, apresenta microespelhos que podem rotar sobre dois eixos, redirecionando desta maneira a luz entre as portas de entrada e saídas, resultando na necessidade de somente $\mathrm{N}$ ou $2 \mathrm{~N}$ microespelhos. A utilização de $2 \mathrm{~N}$ microespelhos é dominante em dispositivos comerciais, pois esta configuração 
proporciona uma menor perda de inserção óptica. Alternativamente, se somente $\mathrm{N}$ microespelhos forem usados, as diferentes distâncias de propagação dos raios de luz entre as portas comutadas tornam tal abordagem dependente do número de portas a serem comutadas. Assim, a arquitetura $3 \mathrm{D}$ com $2 \mathrm{~N}$ microespelhos pode ser escalável para centenas ou alguns milhares de portas com grande uniformidade nas perdas. O movimento dos microespelhos ocorre em vários graus de liberdade, tornado possível por uma inclinação suave e contínua. Aprimorar aspectos como fabricação e desenvolver eficientes algoritmos de controle dos microespelhos são os desafios desta tecnologia.

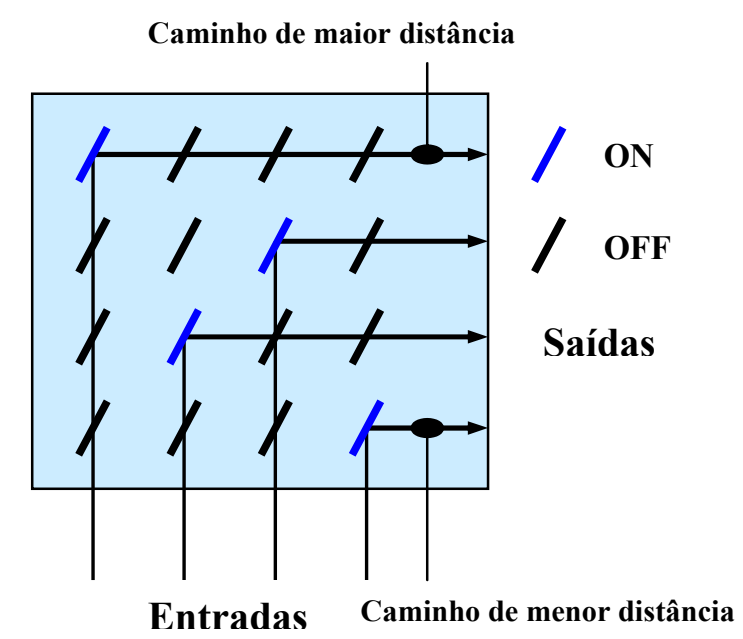

a)

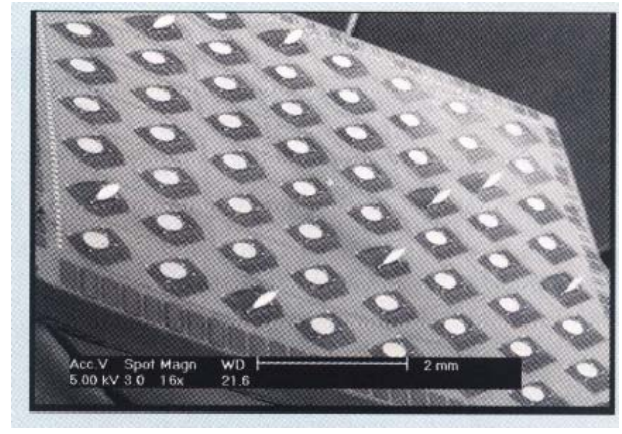

b)

FIGURA 1.6: a) Ilustração esquemática de arquitetura $2 \mathrm{D}$; b) arquitetura $2 \mathrm{D} \mathrm{NxN}$ fabricada pela AT\&T [12].

Em relação ao emprego de novas fibras ópticas, deve-se considerar em qual tipo de rede óptica esta será implantada, pois os novos tipos de fibras apresentam vantagens específicas para cada caso. Esta abordagem aplica-se não somente às redes de longa distância, nas quais as distâncias percorridas pelo feixe de luz variam de centenas a milhares de quilômetros, mas também às redes metropolitanas (dezenas a uma centena de quilômetros) e às redes de acesso (1 a $10 \mathrm{~km}$ ). Em redes de longa distância, empregando sistemas DWDM, torna-se essencial operar com amplificadores ópticos e altas taxas de transmissão por comprimento de onda, barateando assim o custo por bit transmitido. Com este intuito surgiram em 1993 as fibras NZDF (nonzerodisperson fibers), as quais apresentam uma menor dispersão cromática, além de evitar as nãolinearidades presentes nas fibras DS (Dispersion-Shifted). Desde então o uso de fibras NZDF em 
redes de longa distância tem crescido rapidamente. Como exemplo de tais fibras podemos citar a fibra True Wave produzida pela Lucent Technologies e a fibra LEAF produzida pela Corning.

Em se tratando de redes metropolitanas, cujas distâncias típicas são menores que $100 \mathrm{~km}$, amplificadores ópticos raramente são usados e a dispersão cromática da fibra não é um fator limitante. Como este tipo de rede interliga grandes clientes corporativos e inúmeros consumidores, exige-se das fibras ópticas aí instaladas uma grande capacidade de "networking", isto é, flexibilidade para suportar a manipulação de vários comprimentos de onda.

Devido a esta peculiaridade, as redes metropolitanas apresentam características nas quais predomina a possibilidade de inserção e retiradas de comprimentos de onda. A faixa de comprimentos de onda usada pelas fibras monomodos abrange de 1260 a $1650 \mathrm{~nm}$, sendo usualmente empregadas a segunda janela de $1310 \mathrm{~nm}(1280 \mathrm{a} 1325 \mathrm{~nm})$ e a terceira de $1550 \mathrm{~nm}$ (1530 a $1565 \mathrm{~nm})$.

Historicamente, a região de comprimentos de onda entre 1350 e $1450 \mathrm{~nm}$ não tem sido aproveitada devido à atenuação causada pela presença do íon hidroxila $\left(\mathrm{OH}^{-}\right)$, uma impureza residual do processo de fabricação da fibra, cujo pico de absorção está próximo de $1385 \mathrm{~nm}$ [13]. Comercialmente disponível, as chamadas fibras secas eliminam o pico de absorção pelo íon hidroxila, habilitando seu uso para a faixa de 1350 a $1450 \mathrm{~nm}$, incrementando o espectro da fibra por aproximadamente 100nm. Como exemplo podemos citar as fibras All Wave produzidas pela Lucent Technologies.

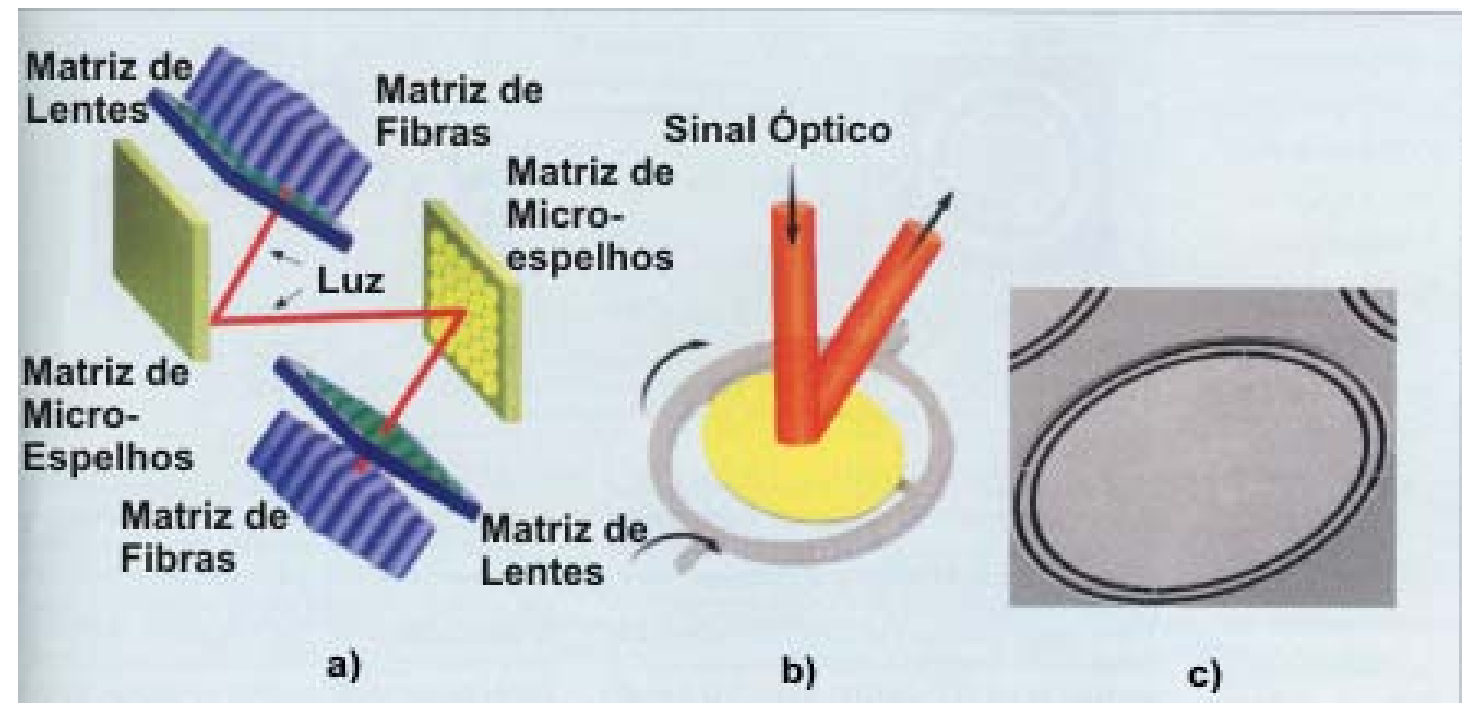

FIGURA 1.7: a) Ilustração de uma arquitetura 3D - 2N; b) Ilustração da reflexão de um feixe de luz usando-se um microespelho com dois eixos de liberdade; c) Protótipo de um microespelho. 
Neste ponto, o conceito e a base tecnológica das redes ópticas foram já adequadamente introduzidos, restando analisar como as aplicações interagirão com esta camada física. Tal interação tem provado ser um desafio dentro da atual arquitetura das redes de dados, no intuito de disponibilizar soluções que habilitem os provedores de serviços a transportar um grande volume de tráfego de uma maneira eficiente em termos de custo e desempenho. Uma das mais utilizadas pilhas de protocolos apresenta tipicamente quatro camadas (Figura 1.8 (a)): IP para a camada de serviço, ATM (asynchronous transfer mode) para a engenharia de tráfego, SONET/SDH para o monitoramento, confiabilidade e restauração e DWDM para o transporte. Infelizmente, esta arquitetura vem se mostrando redundante e incapaz de proporcionar o transporte de grandes volumes de tráfego com eficiência de custo. De fato, arquiteturas multicamadas tipicamente apresentam efeitos nos quais uma camada pode limitar a escalabilidade de redes inteiras, tanto quanto aumentar os custos das mesmas. Com o objetivo de diminuir o número de camadas, na tendência dominante, a função de engenharia de tráfego executada pela camada ATM deverá ser absorvida pela camada IP e a capacidade de transporte do protocolo SONET/SDH (proteção, roteamento e comutação) deverá ser absorvida pela camada óptica, culminando em uma arquitetura com duas camadas, chamada IP sobre DWDM.

A primeira parte deste objetivo pode ser alcançada através da inclusão na camada IP de novas funcionalidades proporcionadas pela tecnologia MPLS (Multiprotocol Label SwitchingInternet Engineering Task Force-IETF) [14] (Figura 1.8 (b)), sendo este um plano de controle que pode ser usado não somente com roteadores, mas também em equipamentos SONET/SDH. A funcionalidade restante pode ser alcançada à medida que comutadores ópticos forem empregados em conjunto com os sistemas DWDM, transformando, ou melhor, dotando, esta camada de meios para realizar a comutação óptica.

A arquitetura com duas camadas (Figura 1.8 (d)) surge assim com a utilização de Generalized MPLS (GMPLS) e a disponibilização de sistemas DWDM com comutação óptica. A Figura 1.9 (a) mostra os vários tipos de redes e a conexão entre as redes de longa distância sendo feitas por comutadores OEOs, cuja função é conectar redes não necessariamente compatíveis entre si, eliminando as distorções introduzidas pelos dispositivos ópticos presentes no caminho do feixe óptico. A arquitetura GMPLS visa prover às redes da Figura 1.9 (b) um único plano de controle, derivado do MPLS, ou seja, algumas modificações e adições são necessárias para que os protocolos de sinalização e roteamento presentes no MPLS se adaptem às peculiaridades da comutação óptica. Desta maneira o GMPLS é estendido para incluir um grupo de elementos de redes que não tomam decisões de comutação e roteamento baseados nas 
informações carregadas nos cabeçalhos de pacotes ou células, mas sim baseados em time slots, comprimento de onda, ou portas. Em outras palavras, a arquitetura de um plano de controle único proporcionado pelo GMPLS assegura a troca de rótulos (labels), enviados de uma porta de entrada para uma porta de saída, em qualquer tipo de nó da rede. Para roteadores IP os rótulos designam principalmente portas de entrada e saída. Para redes ópticas, os rótulos designam portas de entrada e saída e comprimentos de onda ou banda de comprimentos de onda para cada OXC. Para dispositivos de multiplexação por divisão de tempo, equipamentos SONET/SDH, os rótulos designam time slots de entrada e saída [15].

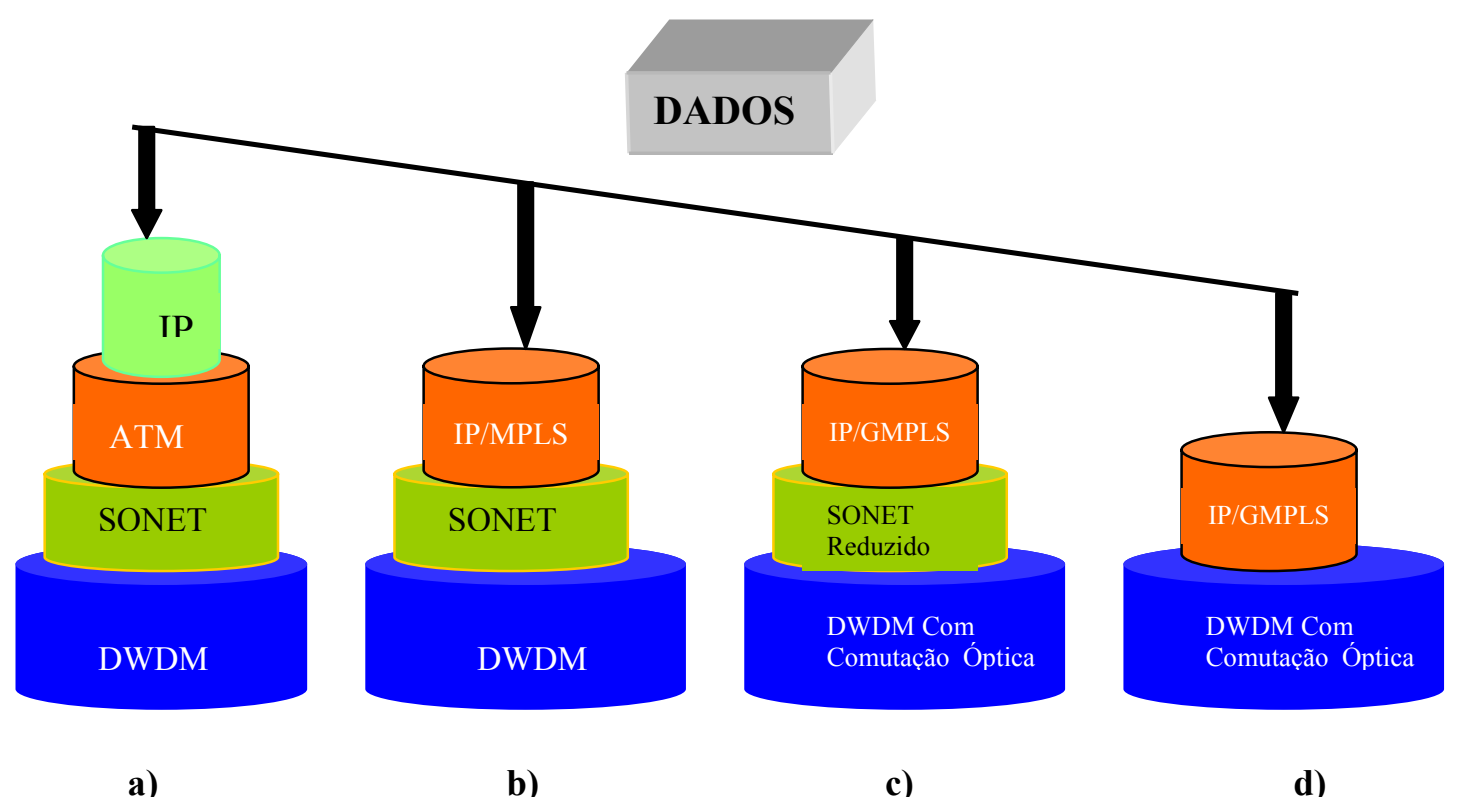

FIGURA 1.8: Evolução em direção a arquitetura de redes ópticas com duas camadas.

Analisar a arquitetura destas redes ópticas, discutir o estado da arte dos protocolos necessários ao seu desenvolvimento, e avaliar os vários esforços empregados para a padronização destas por diferentes instituições são as propostas deste trabalho.

No Capítulo 2 desta dissertação apresentar-se-ão as principais arquiteturas multicamadas disponíveis, discutindo o desempenho destas estruturas através do cálculo do overhead introduzido na sua utilização. No Capítulo 3 são apresentados os conceitos básicos de roteamento e protocolos envolvidos na arquitetura IP/MPLS, com atenção especial à engenharia de tráfego proporcionado pela tecnologia MPLS. Apresentar a evolução da arquitetura MPLS para o Generalized MPLS (GMPLS) padronizado pelo IETF é a proposta do Capítulo 4. No 
Capítulo 5 apresenta-se a versão para arquitetura de redes ópticas do ITU, e a inserção da arquitetura GMPLS nesta padronização. Finalmente, no Capítulo 6 são apresentadas as conclusões sobre esta comparação.

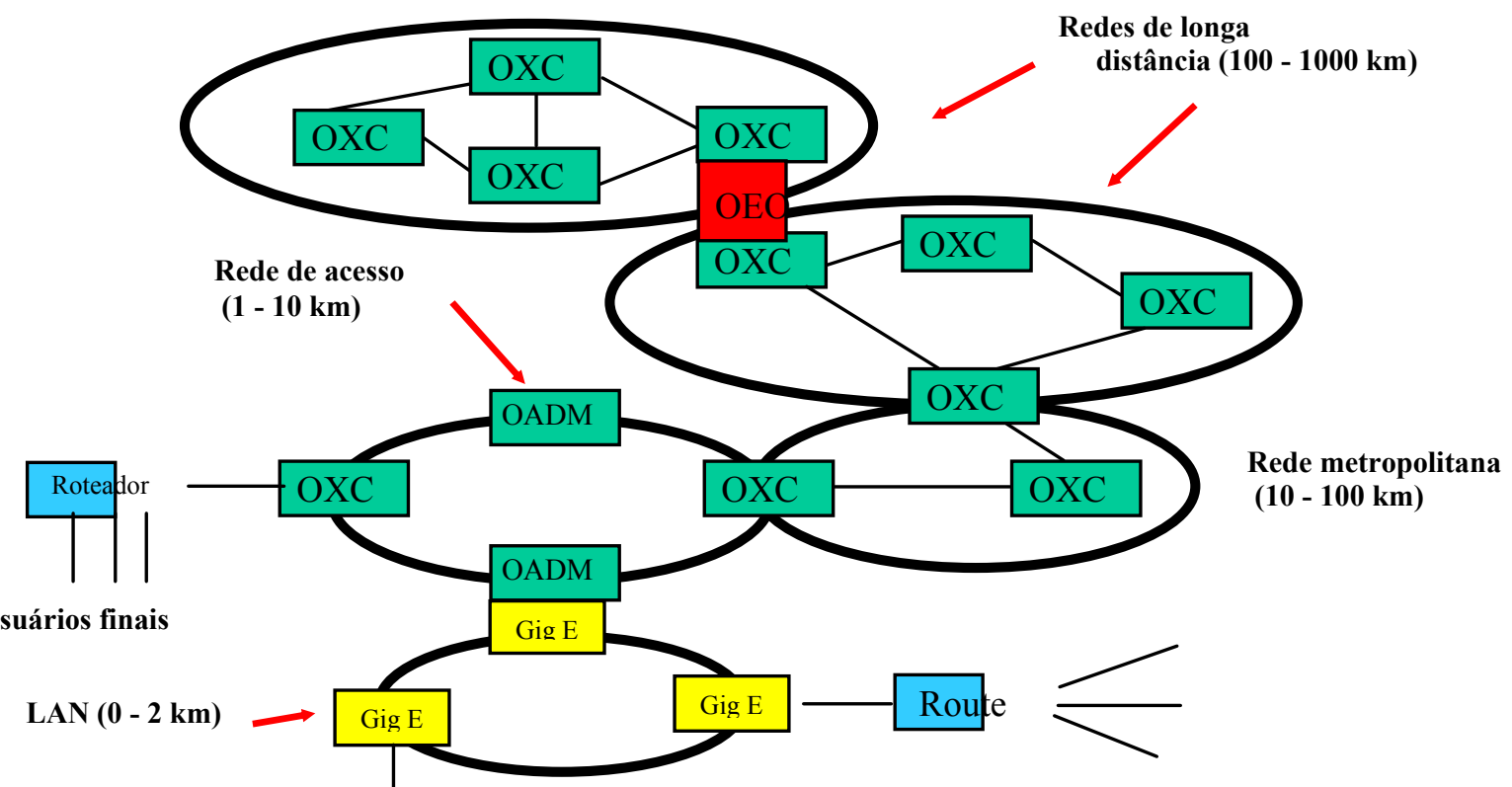

a)

Route $=$

Funcões de controle $=$ Plano de controle comum GMPLS

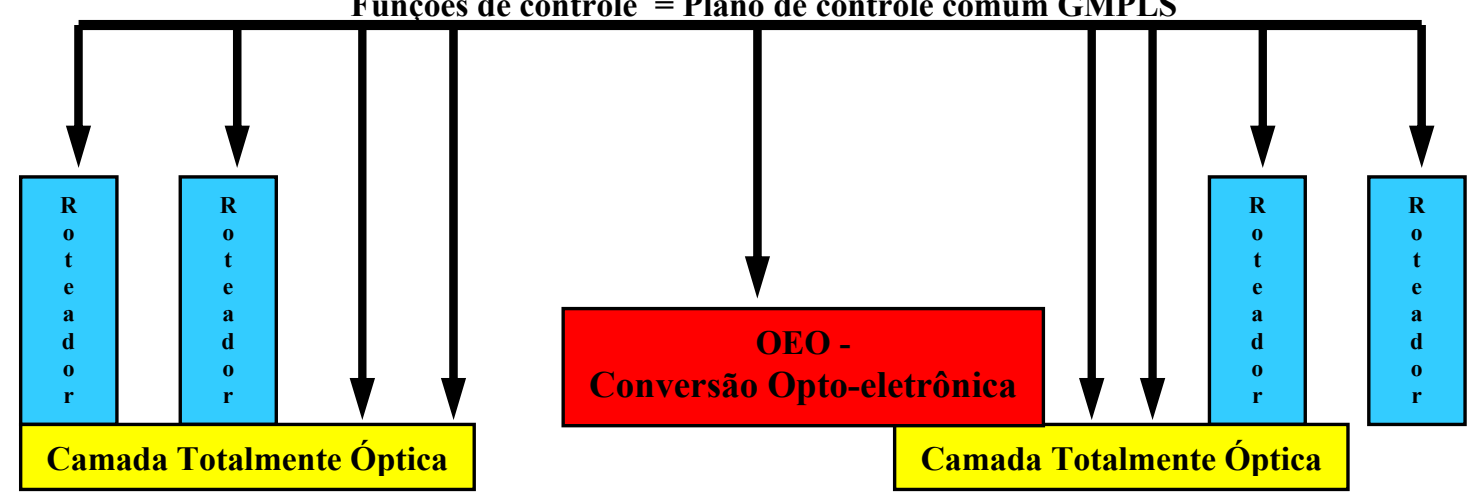

b)

FIGURA 1.9: a) Redes Ópticas com sub-redes totalmente transparentes conectadas umas às outras através de OEOs e usuários conectados via roteadores; b) Plano de controle comum, visão GMPLS. 


\title{
CAPÍTULO 2
}

\section{LIMITAÇÕES DE DESEMPENHO DEVIDO À INSERÇÃO DE OVERHEADS DAS PRINCIPAIS ARQUITETURAS MULTICAMADAS}

\author{
Neste capítulo serão apresentadas as pilhas de protocolo IP/ATM/SONET-SDH e \\ IP/SONET-SDH, explicitando a forma pela qual tais arquiteturas multicamadas executam o \\ encapsulamento do protocolo IP. Finalizando, será executado o cálculo do overhead inserido \\ durante o processo de encapsulamento, demonstrando as vantagens existentes na abordagem IP \\ sobre DWDM em comparação com as possibilidades mencionadas acima.
}

\subsection{INTRODUÇÃO}

As arquiteturas multicamadas ilustradas na Figura 2.1 representam diferentes formas de se habilitar IP em redes ópticas. Cada uma delas provê diferentes funcionalidades em termos de escalabilidade, overhead, gerenciamento de tráfego e QoS (qualidade de serviço). Como mencionado, neste capítulo analisou-se as arquiteturas multicamadas IP/ATM/SONET-SDH e IP/SONET-SDH. O protocolo ATM provê gerenciamento de largura de banda baseado em circuitos virtuais, enquanto a infra-estrutura de transporte é de responsabilidade do binômio SONET-SDH, baseado em uma hierarquia TDM. O intuito deste capítulo será demonstrar a alta porcentagem de overhead vinculado a estas arquiteturas, fator este de suma importância quando se deseja aumentar a eficiência de transmissão em tais redes.

A seguir, a Seção 2.2 discute a forma de encapsulamento para a arquitetura multicamada IP/ATM. Na Seção 2.3 é discutida a arquitetura multicamada IP/SONET-SDH. Na Seção 2.4 é analisado o overhead inserido por tais arquiteturas. O capítulo é finalizado na Seção 2.5, na qual os pontos mais importantes são resumidos. 


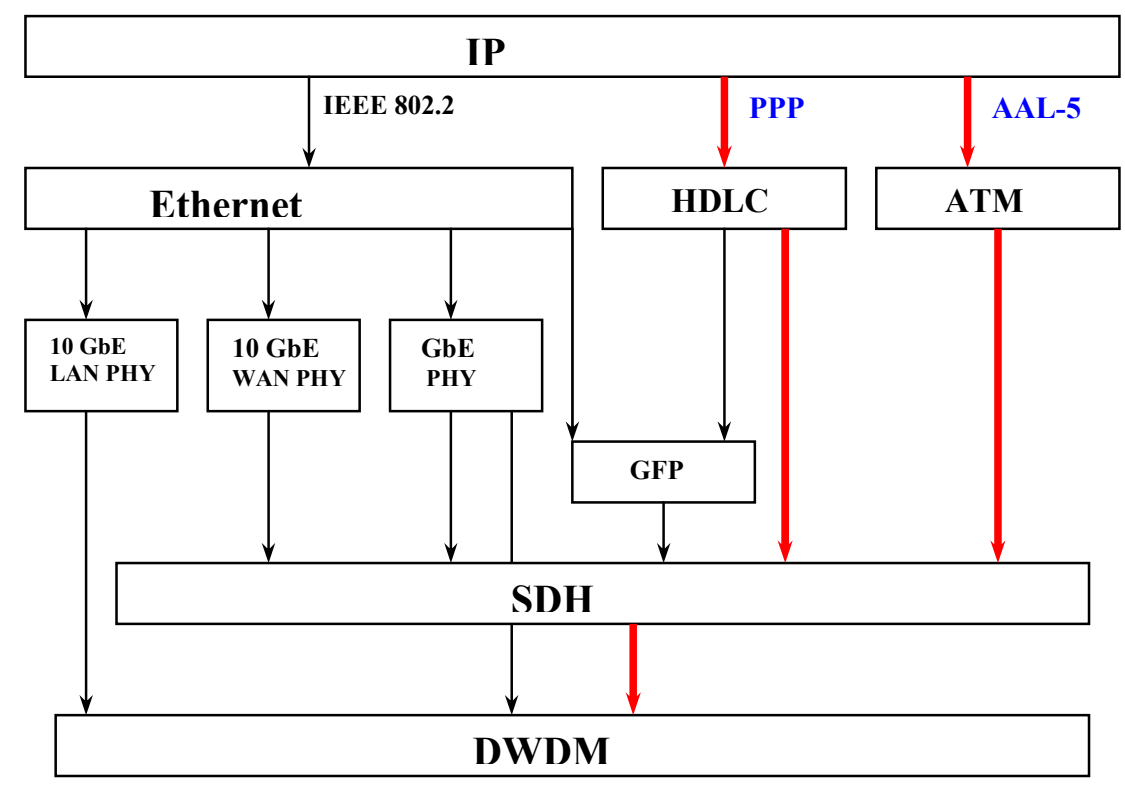

AAL-5 - ATM adaptation layer type 5 GFP - Generic framing procedure
PPP - Point-to-Point Protocol
HDLC - High-level Data Link Control

FIGURA 2.1: Arquiteturas multicamadas para transporte de IP em redes ópticas. As setas em vermelho apresentam as arquiteturas especificadas neste capítulo.

\subsection{IP SOBRE ATM SOBRE SONET/SDH}

Uma configuração comum para transporte IP é IP sobre ATM sobre SONET/SDH, provendo taxas de transmissão de $155 \mathrm{Mb} / \mathrm{s}$ e $622 \mathrm{Mb} / \mathrm{s}$, capacidade de gerenciamento de desempenho e detecção de falhas, assim como restauração, proporcionada pelos protocolos SONET-SDH. Por outro lado, o ATM provê flexível alocação de banda para os enlaces, existindo uma padronização para este encapsulamento [16].

O clássico mapeamento IP sobre ATM sobre SONET-SDH pode ser descrito como um processo de três passos (Figura 2.2):

1-) Cada datagrama IP é encapsulado dentro de um quadro da camada de adaptação tipo 5 (AAL-5-ATM adaptation layer type 5) usando LLC (logical link control) e encapsulamento SNAP (subnetwork attachment point).

2-) O quadro AAL-5 é segmentado em células ATM de 48 bytes de payload.

3-) Células ATM são então mapeadas dentro do quadro SONET ou SDH 


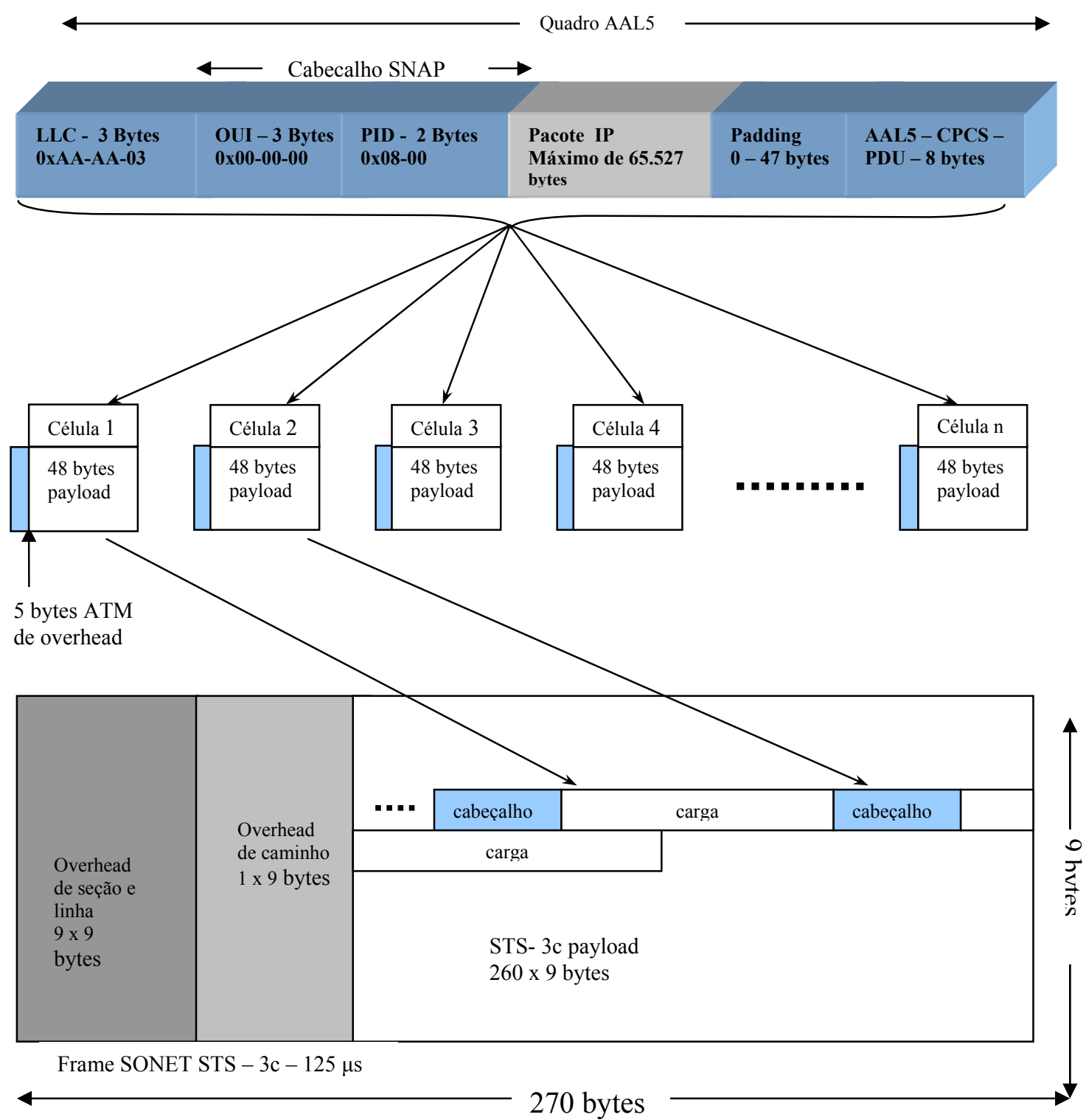

AAL-5 - ATM adaptation layer type 5 ATM - Asynchronous transfer mode

OUI - Organizationally unique identifier CPCS - Common part convergence sublayer PDU - Protocol data unit PID - Protocol identifier IP - Internet Protocol SNAP - Subnetwork attachment point LLC - Logical link control SONET - Synchronous optical network STS - 3c Synchronous transport signal 3 concatenated

FIGURA 2.2: IP sobre ATM sobre SONET. 
Uma descrição detalhada do significado de cada bloco de um quadro AAL-5, conforme indicado na Figura 2.2, consta do Apêndice I.

\subsection{IP SOBRE SONET/SDH}

O mapeamento de pacotes IP diretamente sobre SONET/SDH (Figura 2.3) pode ser entendido como o encapsulamento de pacotes IP em quadros PPP-HDLC (Point-to-Point Protocol /High-level Data Link Control), os quais são transportados por uma infra-estrutura de rede SONET/SDH composta por multiplexadores Add/Drop (ADMs- Add/Drop Multipexer) e comutadores DXCs (Digital Cross-Connects), ou por uma infra-estrutura de DWDM, a qual mapeia quadros SONET/SDH em comprimentos de onda. A padronização de mapeamento para IP sobre SONET/SDH usando PPP-HDLC foi definido pelo IETF na RFC 2615 [17].

Pacotes IP são encapsulados em PPP, o qual foi escolhido como o método padrão para comunicação em enlaces ponto a ponto. Desde que SONET-SDH é, por definição, um circuito ponto a ponto, PPP é adequado para uso sobre estes enlaces. O protocolo PPP descrito na IETF RFC 1661[18] tem como principais características a encapsulação multiprotocolo, detecção de erro e controle de enlace (Link Control Protocol). Os quadros (frames) PPP encapsulando pacotes IP são assim estruturados usando o HDLC de acordo com IETF RFC 2615 e mapeados byte a byte de uma maneira síncrona dentro do envelope de carga síncrono (synchronous payload envelope - SPE/SONET ou virtual container-VC/SDH). A principal função do HDLC é prover o delineamento ou demarcação dos pacotes PPP, quando estes são mapeados em enlaces SONET/SDH. A delineação é realizada usando uma técnica chamada inserção de bytes (byte stuffing). Detalhes desta técnica e das definições dos blocos correspondentes ao encapsulamento PPP-HDLC da Figura 2.3 são dados no Apêndice I.

\subsection{CÁLCULO DE OVERHEAD INTRODUZIDO PELAS VÁRIAS PILHAS DE PROTOCOLOS}

Nesta seção analisaremos o overhead introduzido pelas arquiteturas multicamadas descritas na Figura 2.1. A Tabela 2.1 mostra a distribuição dos cinco predominantes tamanhos de pacotes em um backbone Internet. Os dados, obtidos pelo National Laboratory of Advanced Network Research, consistem de uma amostra de cinco minutos (mais de 18 milhões pacotes, totalizando 
6,7 GB) de um enlace OC-3/STM-1 (155,52 Mbps) presente no backbone da MCI Tecommunications Corporation em junho de 1997 [19]. A Tabela 2.2 mostra a porcentagem de overhead inserida pelas arquiteturas multicamadas IP/PPP/HDLC e a clássica IP/ATM para os cinco tamanhos de pacotes predominantes. O detalhamento dos cálculos de overhead apresentados na Tabela 2.2 consta do Apêndice I. Ressalta-se que o overhead inserido pela infraestrutura de transporte SONET/SDH (aproximadamente 4,6 \%) não está considerado nestes cálculos, sendo descrito separadamente na Tabela 2.3.

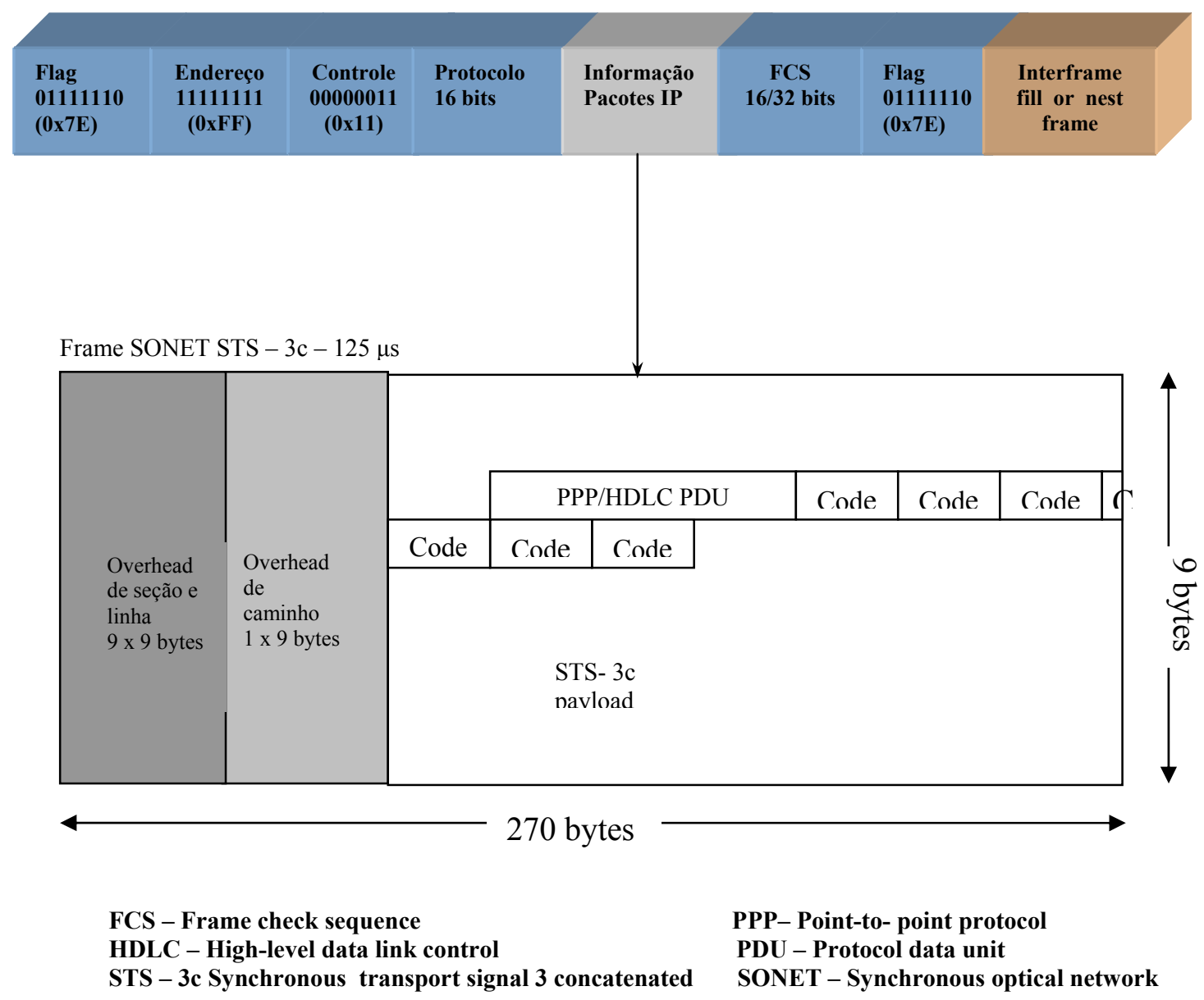

FIGURA 2.3: IP over SONET usando encapsulamento PPP/HDLC. 


\begin{tabular}{|c|c|c|}
\hline & & \\
Tamanho dos Pacotes IP (bytes) & Total de Pacotes (\%) & Total Bytes (\%) \\
\hline 40 & 38,9 & 4,4 \\
\hline 1500 & 11,5 & 48,7 \\
\hline 552 & 10,1 & 15,8 \\
\hline 44 & 6,1 & 0,8 \\
\hline 576 & 4,9 & 7,9 \\
\hline
\end{tabular}

TABELA 2.1:Distribuição de tamanhos de pacotes no backbone Internet da MCI Telecomunication.

\begin{tabular}{|c|c|c|}
\hline $\begin{array}{c}\text { IP } \\
\text { Tamanho de Pacotes (bytes) }\end{array}$ & $\begin{array}{c}\text { IP sobre SONET } \\
\text { Overhead \% }\end{array}$ & $\begin{array}{c}\text { ATM } \\
\text { Overhead \% }\end{array}$ \\
\hline 40 & 20,0 & 165,0 \\
\hline 1500 & 0,5 & 13,1 \\
\hline 552 & 1,4 & 15,2 \\
\hline 44 & 18,2 & 140,9 \\
\hline 576 & 1,4 & 19,6 \\
\hline
\end{tabular}

TABELA 2.2: Porcentagem de overhead de PPP/HDLC e ATM [20].

O overhead inserido pelo encapsulamento IP/ATM constitui-se no overhead de célula (5 bytes de cabeçalho) somado ao overhead inserido pela encapsulação AAL-5.

Da Tabela 2.1 observamos que $45 \%$ de todos os pacotes do backbone Internet apresentam 40 ou 44 bytes. Nenhum deles pode ser encapsulado em uma única célula ATM usando o mapeamento IP/ATM (vide Apêndice I). Como resultado, para estes dois tamanhos de pacotes, o mapeamento IP/ATM é extremamente ineficiente de uma perspectiva de overhead.

O overhead médio ponderado do mapeamento de IP sobre ATM para a distribuição dos tamanhos de pacotes da Tabela 2.1 é de aproximadamente 24\% (vide Apêndice I). Em comparação o overhead médio ponderado para o mapeamento de IP/PPP/HDLC, o qual resulta do acréscimo de fixos 8 bytes de overhead por pacote IP independente do tamanho do pacote a ser transportado, é de aproximadamente 2\% (vide Apêndice I). Ao se planejar backbones onde estará presente o encapsulamento IP/ATM, deve-se levar em conta um overhead de aproximadamente $24 \%$ no cálculo da capacidade do enlace.

\subsubsection{Overhead inserido pela encapsulação SONET/SDH}

Antes de realizar os cálculos do overhead inserido pelo padrão SONET/SDH deve-se discutir em mais detalhes este sistema com o intuito de compreender melhor os vários tipos de overheads existentes. 
A camada física do padrão SONET/SDH é dividida em quatro subcamadas (Figura 2.4), cada uma das quais corresponde a um nível de comunicação entre os equipamentos SONET/SDH. A subcamada mais baixa é a subcamada óptica, a qual se encarrega da especificação das propriedades físicas do feixe óptico e da fibra óptica a serem usadas. As demais são, começando com a mais baixa, camada de seção (regeneradores), camada de linha (multiplexadores) e camada de caminho. A subcamada de seção trata do trajeto ponto a ponto, gerando um quadro padrão em uma extremidade e processando-o na outra. As seções podem começar e terminar nos repetidores, os quais amplificam e geram novamente os bits, não os alterando.

A subcamada de linha é encarregada de multiplexar os diversos tributários em uma única linha e demultiplexá-los na outra extremidade, sendo transparente aos repetidores. A subcamada de caminho trata da conexão entre a origem e destino dos bytes enviados.

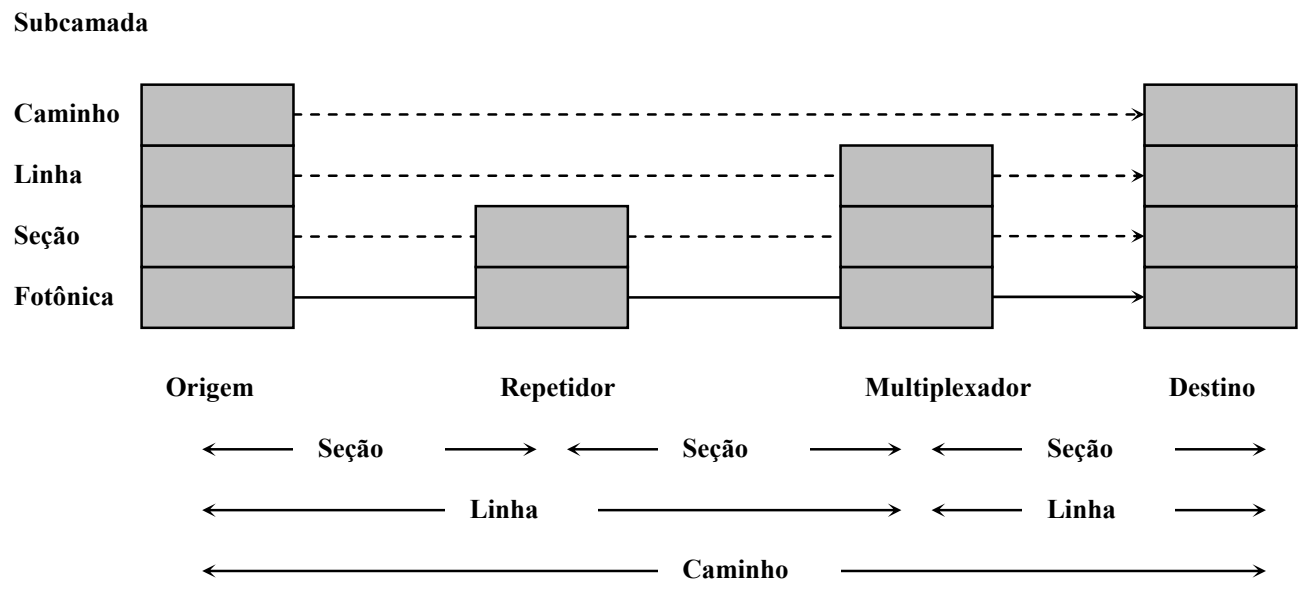

FIGURA 2.4: Subcamadas da camada física do padrão SONET/SDH.

O quadro básico do padrão SONET (Figura 2.5) é formado por um bloco de 810 bytes, transmitido a cada $125 \mu \mathrm{s}$, da esquerda para a direita e de cima para baixo, de forma síncrona, independente de haver ou não dados úteis a serem enviados. A taxa de 8000 quadros/s corresponde exatamente à taxa de amostragem dos canais PCM (pulse code modulation) usados em todos os sistemas de telefonia digital.

O quadro de 810 bytes é formado por uma matriz de bytes composto por 90 colunas de largura por 9 linhas de altura. Desta maneira tem-se 6480 bits $(810 \times 8$ bytes $)$ transmitidos 8000 vezes por segundo, para uma taxa bruta de $51,84 \mathrm{Mbps}$, sendo este o canal básico do padrão 
SONET, chamado de STS-1 (Synchronous Transport Signal-1), do qual todos os outros troncos são múltiplos, incluindo-se os pertencentes ao padrão SDH.

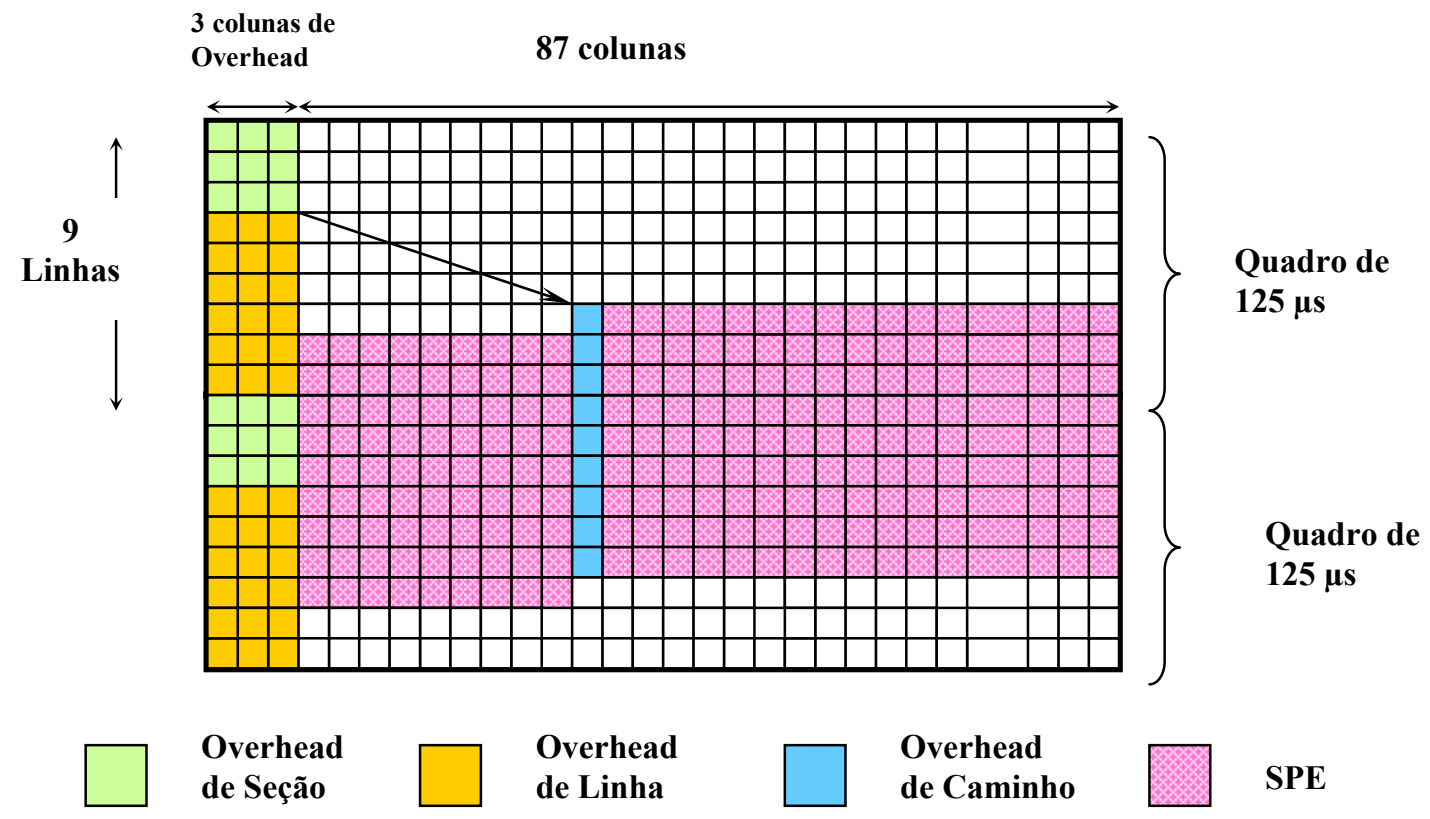

FIGURA 2.5: Quadro básico do padrão SONET.

As três primeiras colunas de cada quadro são reservadas às informações de gerenciamento do sistema. As três primeiras linhas contêm o overhead de seção; as seis linhas seguintes contêm o overhead de linha ou overhead de seção multiplexadora. As 87 colunas restantes contêm 50,112 Mbps dos dados dos usuário $(87 \times 9 \times 8 \times 8000)$. Entretanto, os dados do usuário, chamado SPESONET/VC-SDH (synchronous payload envelope/ virtual container) nem sempre começam na linha 1 , coluna 4 , podendo, sim, começar em qualquer lugar do quadro. A primeira linha do overhead de linha contêm um ponteiro, o qual aponta para o primeiro byte. A primeira coluna do SPE/VC é o overhead de caminho, ou seja, o cabeçalho do protocolo da subcamada de caminho.

Por ser utilizado no Brasil o padrão SDH será descrito quanto ao quadro básico, os tipos de overheads inseridos e a forma de multiplexação existente. A Figura 2.6 mostra o quadro básico da hierarquia SDH denominado STM-1 (Módulo de transporte síncrono) cuja taxa de transmissão é de 155,52 Mbps, bem como os tipos de overhead existentes. 


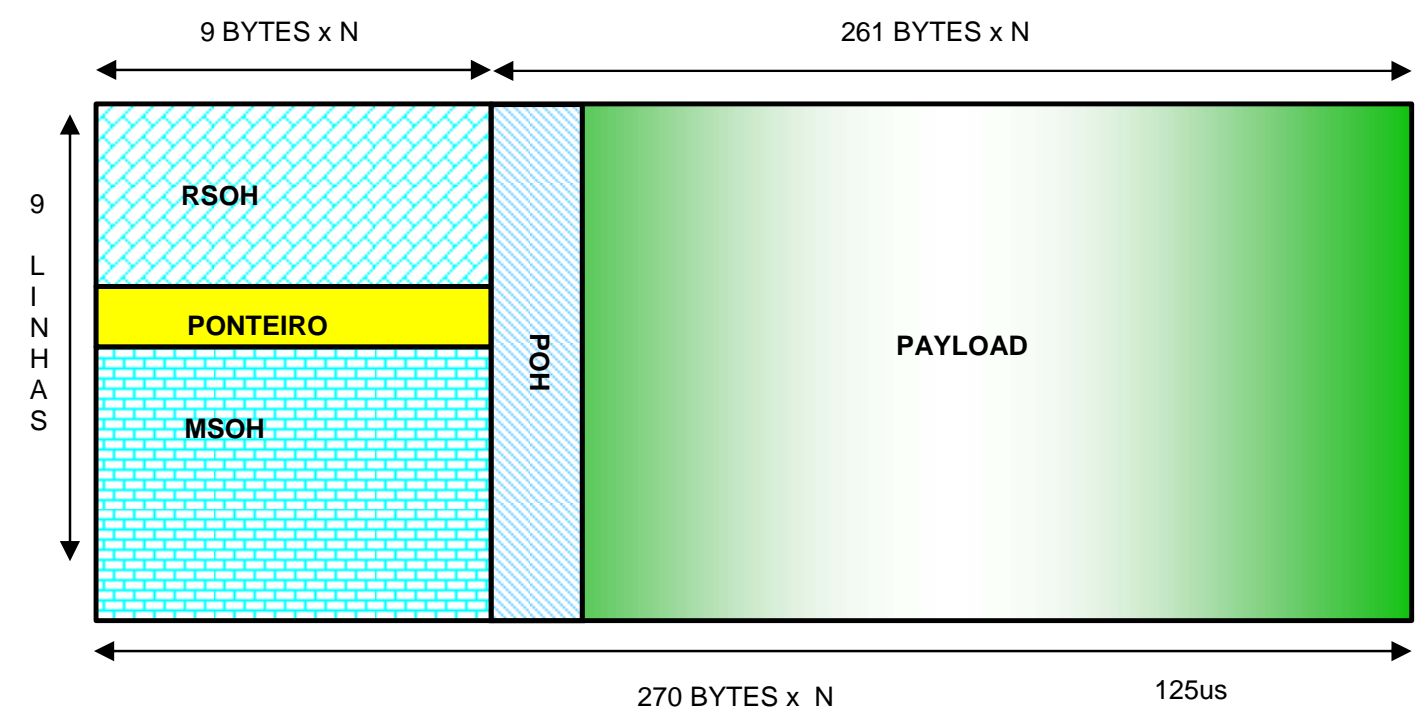

RSOH - Overhead da Seção Regeneradora

MSOH - Overhead da Seção Multiplexadora

POH - Overhead de Via

FIGURA 2.6: Quadro básico do padrão SDH com os quatro tipos de overhead.

São quatro os tipos de overhead existentes na hierarquia SDH:

1. Overhead de Seção

O overhead de Seção (SOH) é a informação adicionada ao payload para criar um STM-

1. O SOH é dividido em RSOH (Overhead da Seção Regeneradora) e MSOH (Overhead da Seção Multiplexadora) e fornece informações de alinhamento de quadro, manutenção e outras funções operacionais. A informação do MSOH passa transparentemente através dos regeneradores, sendo utilizada na Camada de Seção Multiplexadora onde os AUGs (Grupo de Unidades Administrativas) são montados.

2. Overhead de Via (POH)

O POH é a informação adicionada ao "payload" para criar um VC, sendo dividido em HO POH (Overhead da Via Superior) e LO POH (Overhead da Via Inferior). Esta informação provê a integridade da comunicação no caminho percorrido dentro da Camada de Via, ou seja, entre os pontos onde são montados e desmontados os VCs. O 
LO POH é introduzido quando se monta um VC de ordem inferior (VC-12 e VC-3) e o $\mathrm{HO}$ POH é introduzido quando se monta um VC de ordem superior (VC-4). Os ponteiros indicam o byte onde o POH tem início.

A multiplexação de vários fluxos de dados, chamados de tributários, desempenha um papel importante nos padrões SONET e SDH. A Figura 2.7 apresenta a estrutura simplificada da hierarquia SDH e os processos de mapeamento, multiplexação e alinhamento decorrentes deste padrão.

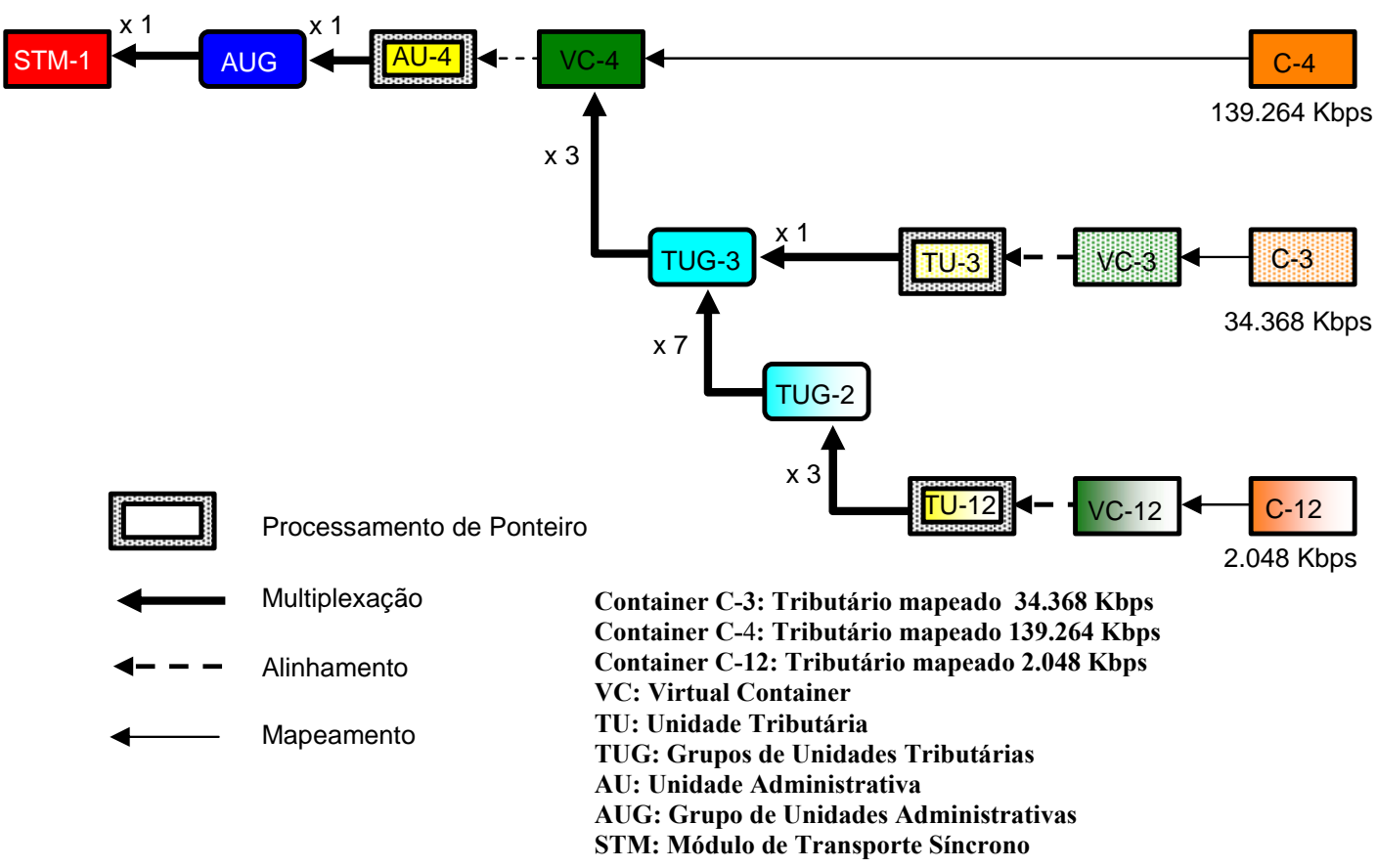

FIGURA 2.7: Estrutura simplificada da hierarquia SDH.

O mapeamento é, portanto, o processo pelo qual tributários são adaptados em VCs. O processo pelo qual sinais da camada de via de ordem inferior são adaptados na camada de via de ordem superior ou sinais da camada de via de ordem superior são adaptados na camada de seção multiplexadora é denominado multiplexação. A TU (unidade tributária) é a estrutura de informação que provê adaptação entre a Camada de Via de Ordem Inferior e a Camada de Via de Ordem Superior. O entrelaçamento byte a byte de Tus dá origem às TUGs (grupo de unidades tributárias). A AU (unidade administrativa) é a estrutura de informação que provê a adaptação 
entre a Camada de Via de Ordem Superior e a Camada de Seção multiplexadora. A AUG (grupo de unidades administrativas) é uma estrutura de informação constituída de uma AU-4. Já o alinhamento é processo pelo qual a informação de "offset", é incorporada ao ponteiro de TU ou AU. Por exemplo, o ponteiro de uma TU-3 indica, através de um "offset" de quadro, em que byte da estrutura de TU-3 se encontra o primeiro byte do POH do VC-3.

A hierarquia da SONET/SDH é mostrada na Tabela 2.3 na qual foram definidas taxas de STS-1 até STS-48, por serem as mais utilizadas. A OC (optical carrier) de comunicações que corresponde a STS-n é chamada de OC-N, possuindo a mesma configuração bit a bit. Os nomes SDH são diferentes, pois não possuem a taxa básica de 51,84 Mbps. As taxas brutas incluem todo o overhead. A taxa de dado SPE exclui o overhead de seção e de linha. A taxa de dados do usuário exclui todo o overhead e inclui somente as 86 colunas disponíveis para a carga útil.

Todavia, quando um STM-N, tal como uma STM-4, não é multiplexado, mas transporta os dados de uma única origem, a letra c (de concatenado) é acrescentada à designação. O volume de dados do usuário em um fluxo STM-4c é levemente mais alto do que em um fluxo STM-4, pois a coluna de overhead de caminho é incluída no SPE somente uma vez e não quatro como acontece com os quatros fluxos STM-1 independentes. O processo de concatenação não foi considerado na confecção desta tabela.

\begin{tabular}{|c|c|c|c|c|c|}
\hline \multicolumn{2}{|c|}{ SONET } & SDH & \multicolumn{3}{c|}{ Taxa de dados (Mbps) } \\
\hline Elétrica & Óptica & Óptica & Bruta & SPE & Usuário \\
\hline STS-1 & OC-1 & & 51,84 & 50,112 & 49,536 \\
\hline STS-3 & OC-3 & STM-1 & 155,52 & 150,336 & 148,608 \\
\hline STS-9 & OC-9 & STM-3 & 466,56 & 451,008 & 445,824 \\
\hline STS-12 & OC-12 & STM-4 & 622,08 & 601,344 & 594,432 \\
\hline STS-18 & OC-18 & STM-6 & 933,12 & 902,016 & 891,648 \\
\hline STS-24 & OC-24 & STM-8 & 1244,16 & 1202,688 & 1188,864 \\
\hline STS-36 & OC-36 & STM-12 & 1866,24 & 1804,032 & 1783,296 \\
\hline STS-48 & OC-48 & STM-16 & 2488,32 & 2405,376 & 2377,728 \\
\hline
\end{tabular}

STS - Synchronous Transport Signal

OC - Optical Carrier

STM - Módulo de transporte síncrono

SPE - Synchronous Payload Envelope

TABELA 2.3: Taxas de multiplexação e overhead da hierarquia SONET e SDH.

Analisando a taxa de overhead inserida pelo padrão SONET/SDH calcula-se uma taxa de overhead médio de $4,6 \%$. 


\subsection{COMENTÁRIOS E CONCLUSÕES}

Até este ponto analisamos uma das variáveis, a taxa de overhead, referente as arquiteturas multicamadas descritas acima. Uma análise qualitativa das referidas pilhas de protocolos impõe outros ângulos de observação.

A arquitetura multicamada IP/ATM introduz um overhead médio de aproximadamente $24 \%$, adicionados aos 4,6\% de overhead proporcionados pelo padrão SONET/SDH. Além disso, a necessidade de construir e gerenciar duas redes com tecnologia dissimilar (IP e ATM), torna-se um obstáculo a este método de encapsulamento. No entanto, o ATM permite um método de engenharia de tráfego e um particionamento da largura de banda do padrão SONET/SDH [21]. Muitos operadores de redes ainda consideram esta característica importante o suficiente para compensar o overhead inserido por tal arquitetura.

A solução IP/PPP/HDLC/SONET/SDH provê uma maneira de otimizar a utilização da banda de transmissão por acrescentar uma taxa de overhead de aproximadamente $2 \%$, adicionados também aos 4,6\% de overhead proporcionados pelo padrão SONET/SDH. Além disso, a disponibilização da tecnologia MPLS, com sua capacidade de gerenciamento de tráfego, provavelmente cobrirá a perda da flexibilidade de alocação de canais virtuais e poderá ser empregada na substituição da camada ATM. Por esta razão o conceito básico desta tecnologia será o assunto do próximo capítulo. 


\title{
CAPÍTULO 3
}

\section{APLICAÇÃO DA ARQUITETURA MPLS NA IMPLEMENTAÇÃO DE ENGENHARIA DE TRÁFEGO EM REDES IP}

\begin{abstract}
Neste capítulo será analisada a aplicação da arquitetura MPLS com o intuito de viabilizar a implementação de engenharia de tráfego em redes IP. Com este objetivo serão apresentados os conceitos básicos desta arquitetura. A seguir, a topologia de redes MPLS será explicitada e comparada às redes IP. Finalizando, será demonstrado como, através de tais conceitos básicos, se pode dotar redes IP com a propriedade de engenharia de tráfego.
\end{abstract}

\subsection{INTRODUÇÃO}

A engenharia de tráfego tem como objetivo a otimização do desempenho operacional de uma rede. Otimização esta imposta pela necessidade de tornar a rede mais rápida, confiável e eficiente em termos de custo. Engenharia de tráfego significa mapear de maneira eficiente fluxos de tráfego IP nos recursos físicos existentes em uma rede, enquanto simultaneamente atende-se às exigências de qualidade (atrasos, níveis de proteção) do transporte desejado pelas informações contidas nos pacotes. Existem técnicas para realizar este objetivo, uma delas é a arquitetura MPLS. A idéia básica desta arquitetura consiste em designar rótulos aos pacotes na borda de uma rede MPLS. No interior do domínio MPLS os rótulos anexados aos pacotes são usados para tomar decisões de envio, sem a necessidade de se analisar o cabeçalho original dos referidos pacotes. Desta maneira, a transmissão de dados ocorre nos LSPs (label switched paths), os quais são uma seqüência de rótulos designados em cada nó ao longo do caminho da fonte para o destino. Os rótulos são distribuídos usando-se protocolos de distribuição como o CR-LDP (Constraint-based Routing with Label Distribution Protocol) ou o RSVP (Resource Reservation Protocol) ou carregados em protocolos de roteamento como o BGP (Border Gateway Protocol). A escolha recai sobre aquele que melhor atender as exigências do operador da rede, não sendo 
imposta pela arquitetura MPLS [14]. Cada pacote encapsula e carrega o rótulo durante sua jornada da fonte ao destino. O processamento rápido dos pacotes (comutação) ocorre devido ao fato de não haver necessidade de consulta ao cabeçalho do pacote para comutá-lo entre enlaces. Os LSPs podem ser configurados de uma maneira explícita antes de ocorrer a transmissão de dados, ou seja, os nós pelos quais os pacotes trafegarão são especificados a priori. Ao longo do caminho os recursos podem ser reservados para assegurar QoS (Qualidade de serviço) para este tráfego de dados. Tal característica possibilita a engenharia de tráfego através da rede, pois permite a utilização de outros caminhos que não os caminhos mais curtos usualmente selecionados pelos protocolos de roteamento IP, alcançando desta forma uma otimização dos recursos da rede. Por exemplo, pode-se mover o fluxo de dados para uma nova rota se o caminho mais curto entre dois nós estiver congestionado. Alternativamente, as características desejadas de um LSP, tais como largura de banda, destino, prioridade, podem ser configuradas em um roteador, o qual usa um algoritmo apropriado para computar o caminho através da rede que satisfaça restrições impostas. O MPLS torna-se desta maneira um instrumento apropriado para estabelecer e manter caminhos explicitamente roteados em uma rede IP para uma efetiva disposição de tráfego.

Os objetivos do grupo de trabalho em MPLS do IETF incluem a padronização de um conjunto de protocolos para a distribuição e manutenção de rótulos para a comutação de pacotes, bem como dos procedimentos para empregar esta tecnologia sobre várias tecnologias de transmissão (SONET/SDH). A meta é melhorar a escalabilidade da rede, proporcionar meios para engenharia de tráfego e fornecer uma relação custo/beneficio aceitável para os provedores de serviços. Esta característica da tecnologia MPLS, na qual o pacote é designado para uma classe equivalente de envio FEC (forward equivalence class) através de um rótulo e todo o processamento de envio realizado nos nós de redes subseqüentes é realizado através do processamento deste, apresenta uma série de vantagens:

1) O processamento de envio de pacotes pode ser feito por comutadores (switches), os quais não são capazes de analisar o cabeçalho da camada de rede (ou pelo menos não são capazes de analisar os cabeçalhos da camada de rede na velocidade adequada); 
2) Quando um pacote é designado a uma FEC na entrada de uma rede, o roteador de ingresso pode usar na referida determinação qualquer informação disponível sobre o pacote, mesmo que a informação não possa ser obtida diretamente do respectivo cabeçalho. Por exemplo, pacotes chegando em diferentes portas podem ser designados para diferentes FECs. O processamento de envio em redes convencionais, por outro lado, pode somente considerar informações contidas no próprio cabeçalho do pacote;

3) Um pacote cuja entrada na rede é realizada por um roteador particular poderia ser rotulado diferentemente se o mesmo pacote entrasse na rede por um roteador diferente. Como resultado, decisões de envio que levam em conta o roteador de ingresso podem ser aplicadas. Isto não ocorre em redes IP convencionais, pois a decisão de envio não leva em conta o roteador de ingresso de um pacote na rede;

4) Algumas vezes é desejável forçar o pacote a seguir uma rota particular explicitamente escolhida antes do pacote entrar na rede, ao invés de determiná-la através de um algoritmo de roteamento dinâmico enquanto o pacote trafega através da rede. Este procedimento pode ser utilizado para engenharia de tráfego;

O termo multiprotocol em MPLS significa que esta tecnologia pode ser usada sob qualquer protocolo de rede (IP, IPX), sendo esta arquitetura padronizada, como visto, pelo IETF. Nesta dissertação, entretanto, o escopo deste estudo basear-se-á no protocolo de rede IP. A seguir, na Seção 3.2 serão apresentados os conceitos básicos da arquitetura MPLS voltados a prover engenharia de tráfego em redes IP. Na Seção 3.3 apresentar-se-á o modo de operação de redes MPLS em comparação às redes IP. A maneira como uma rede MPLS é composta e quais os protocolos presentes, com ênfase nos protocolos de sinalização CR-LDP e RSVP serão apresentados na Seção 3.4. Como os conceitos básicos da tecnologia MPLS podem prover engenharia de tráfego em redes IP é demonstrado na Seção 3.5.

\subsection{CONCEITOS BÁSICOS DA ARQUITETURA MPLS}

Nesta seção, introduzir-se-á alguns conceitos básicos da arquitetura MPLS, como descrito abaixo [14]: 
1. Equipamentos de comutação de rótulos utilizados no núcleo (core label switch routers LSRs) e na borda (edge label switch routers - LSRs);

2. FEC (forward equivalence class);

3. Rótulo (label) e atribuição de rótulo (label binding);

4. LSRs upstream e downstream;

5. Protocolos de distribuição de rótulos;

6. Pilhas de rótulos (label stack);

7. Caminhos comutados por rótulos (LSP - label switched path);

8. Rótulos de entrada inválidos (invalid incoming labels);

9. Túneis LSP;

10. Agregação;

11. Roteamento explícito e hop by hop;

\subsubsection{LSRs de borda e LSRs de núcleo}

Redes utilizando MPLS empregam LSRs de núcleo (core LSRs) e LSRs de borda (edge LSRs). O primeiro é um equipamento de comutação (por exemplo, roteadores IP, switches ATM) situado no núcleo de uma rede MPLS que participa no estabelecimento dos LSPs comutando a seguir o tráfego de dados através das rotas estabelecidas. Em outras palavras, a função de um LSR de núcleo é encaminhar um pacote com base no rótulo deste. Ao receber um pacote, um LSR de núcleo analisa o rótulo, troca-o pelo rótulo apropriado e o encaminha para o próximo LSR.

O segundo é um equipamento situado na borda de uma rede MPLS. Um LSR de ingresso (ingress LSR) é o dispositivo pelo qual um pacote entra na rede MPLS, um LSR de egresso (egress LSR) é o dispositivo pelo qual um pacote deixa a rede MPLS. Tais equipamentos conectam sub-redes (tais como IP e ATM) à rede MPLS, sendo responsáveis pela designação e retirada de rótulos para o tráfego que entra ou sai de uma rede MPLS. A composição completa de uma rede MPLS, com os respectivos equipamentos envolvidos será apresentada na Seção 3.4. 


\subsubsection{FEC (forward equivalence class)}

Uma FEC consiste em uma representação de um conjunto de pacotes, os quais compartilham dos mesmos requisitos de transporte, ou seja, serão encaminhados da mesma maneira em uma rede MPLS. Pacotes de um mesmo fluxo de dados geralmente pertencem à mesma FEC. Requisitos de QoS também podem ser definidos com a designação de FECs. A FEC é representada por um rótulo, e cada LSP é associado a uma FEC. Ao receber um pacote, o LSR de borda verifica a qual FEC ele pertence e o encaminha através do LSP correspondente. Portanto há uma associação pacote-rótulo-FEC-LSP. A associação pacote-FEC acontece apenas uma vez, quando o pacote entra na rede MPLS. Isto proporciona grande flexibilidade e escalabilidade a este tipo de rede.

\subsubsection{Rótulo (label) e atribuição de rótulo (label binding)}

Um rótulo é um identificador, de comprimento fixo, com significado local, sendo usado para identificar uma FEC para a qual um pacote é designado. Mais comumente, um pacote é designado para uma FEC com base em seu endereço de destino proveniente da camada rede. Entretanto, o rótulo nunca é somente uma codificação deste endereço. Uma vez que o pacote tenha sido vinculado a uma FEC, isto é, rotulado (label binding), o transporte do pacote através da rede é baseado na comutação dos rótulos. A decisão de designar rótulos pode ser baseada em critérios de envio com a finalidade de proporcionar engenharia de tráfego.

Em alguns casos, o rótulo reside em um campo do pacote criado especificamente para esta finalidade ou em um campo qualquer já existente no cabeçalho de rede ou de enlace, desde que este esteja disponível [22]. Existem alguns meios distintos de transportar um rótulo em um pacote (Figura 3.1).

$\mathrm{Na}$ encapsulação genérica, a qual pode ser usada com qualquer tipo de enlace, o rótulo é transportado em um cabeçalho extra aplicado em cada pacote entre o cabeçalho da camada de enlace de dados e o cabeçalho da camada 3 (camada de rede). Em enlaces que suportam o conceito de canal virtual, o rótulo pode ser carregado como informação do canal virtual, tipicamente no cabeçalho da camada 2. Por exemplo, em enlaces ATM, o rótulo pode ser carregado no campo VPI (virtual circuit identifier) /VCI (virtual path identifier) aplicado a cada célula do pacote. Já em enlaces frame relay, o rótulo pode ser carregado no campo DLCI (data 
link connection identifier) aplicado a cada pacote. Vários outros tipos de rótulos podem ser usados em redes ópticas e serão vistos posteriormente no capítulo 4.

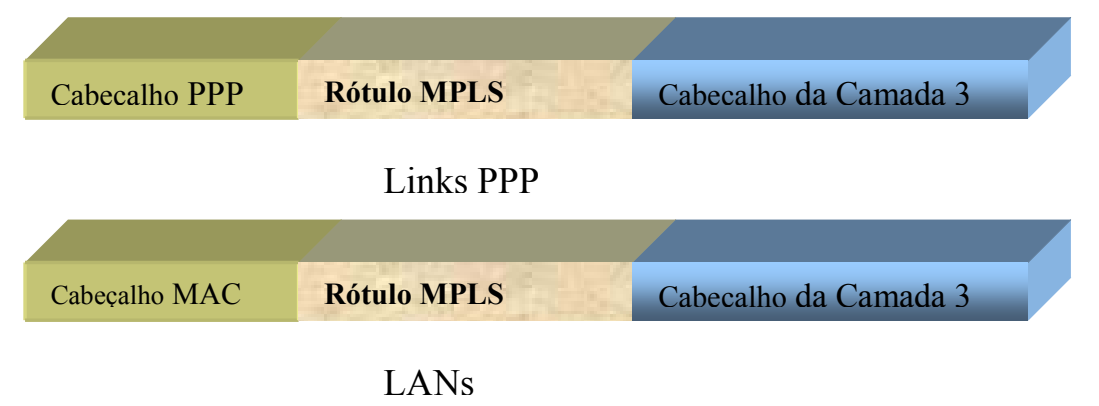

a) Encapsulação de Rótulo Genérica

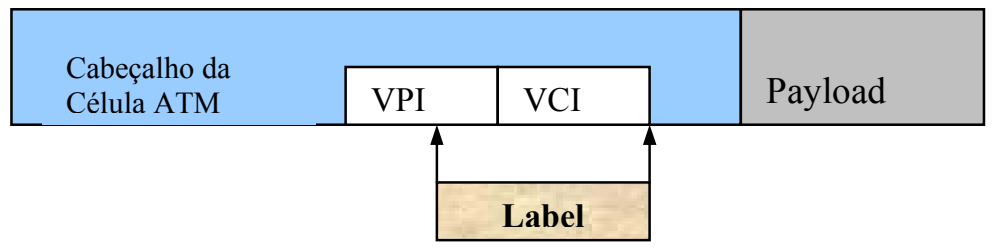

Links ATM

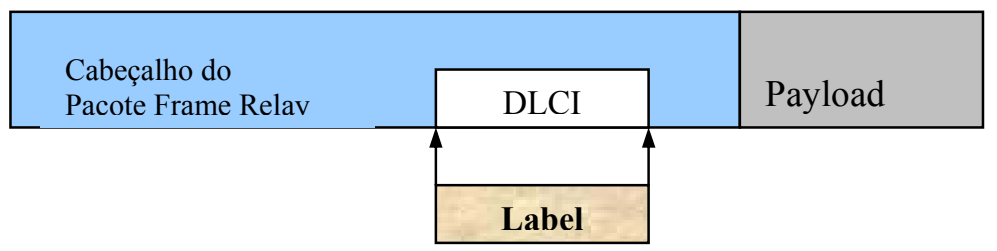

Links Frame Relav

b) Encapsulação de label da camada 2

FIGURA 3.1: Formas de transportar um rótulo em um pacote: a) Encapsulação genérica; b) Encapsulação de label da camada 2.

\subsubsection{LSRs upstream e downstream}

Suponha que dois $\mathrm{LSRs}_{u}$ e $\mathrm{R}_{d}$ concordem em usar um rótulo $\mathrm{L}$ atribuindo-o a uma FEC F para indicar o envio de pacotes do LSR $\mathrm{R}_{u}$ para o LSR $\mathrm{R}_{d}$. O LSR emissor $\mathrm{R}_{u}$ propaga contra o fluxo (upstream) de transmissão, e o LSR receptor $\mathrm{R}_{d}$ é considerado estar a favor do fluxo (downstream). Na arquitetura MPLS, a decisão de atribuir um particular rótulo L a uma 
FEC F é feita pelo LSR que esteja "downstream" com respeito àquela atribuição, sendo deste a responsabilidade de informar o LSR "upstream" sobre tal atribuição. Assim os rótulos são designados "downstream" e distribuídos na direção "downstream-upstream”. Este conceito está especificado na Figura 3.4.

\subsubsection{Protocolos de distribuição de rótulos}

Um protocolo de distribuição de rótulos é um conjunto de procedimentos pelos quais um LSR informa outro da atribuição rótulo/FEC efetuada por ele. A arquitetura MPLS não assume que exista apenas um simples protocolo de distribuição de rótulos. De fato, inúmeros protocolos de distribuição de rótulos estão sendo padronizados. Protocolos de roteamento, tais como o protocolo BGP (border gateway protocol) têm sido estendidos para carregar a informação de rótulo, objetivando a criação de VPNs (virtual private networks) [23]. O protocolo RSVP está também sendo estendido para possibilitar a troca de rótulos [24]. O IETF definiu também um novo protocolo conhecido como LDP (Label Distribution Protocol) para sinalizar e gerenciar explicitamente a troca de rótulos [25]. Extensões para o protocolo LDP têm sido definidas para suportar roteamento explícito baseado em QoS ou outros tipos de restrições (restrições de rotas). $\mathrm{Ou}$ seja, estas extensões oferecem a oportunidade para estender a configuração de rotas (caminhos) utilizando-se informações além daquelas disponibilizadas pelos protocolos de roteamento. Tais definições (CR - constraint-based routing) são incorporadas ao protocolo LDP e referenciadas como CR-LDP [26].

\subsubsection{Pilhas de Rótulos (Label Stack)}

Um pacote pode carregar vários rótulos, organizados em uma pilha (label stack) na qual o último a entrar é o primeiro a sair. Embora a tecnologia MPLS suporte esta hierarquia de rótulos, o processamento destes baseia-se no rótulo do topo da pilha, sem considerar a possibilidade de que alguns rótulos possam ter estado "acima dele" no passado ou que alguns rótulos possam estar "abaixo dele" no presente.

\subsubsection{Codificando uma pilha de rótulos}

Uma pilha de rótulos é representada como uma seqüência de entradas para esta pilha. Cada entrada é representada por 4 bytes. Em outras palavras, para se formar uma pilha de rótulos 
utiliza-se o formato do rótulo MPLS genérico. Cada entrada da referida pilha de rótulos caracteriza-se pela inserção de um rótulo genérico, no cabeçalho do pacote tal qual,o ilustrado na Figura 3.1 a). O último rótulo desta pilha difere dos demais por ter o bit "fim da pilha" codificado para 1, enquanto os demais apresentaram este bit configurado com 0. A Figura 3.2 descreve o rótulo MPLS genérico (4 bytes) e o significado de seus campos [27]:

a) Fim da pilha (Bottom of Stack)

Este bit é codificado para 1 se o rótulo for o último de uma pilha de rótulos e 0 para todos os outros rótulos da pilha

b) Tempo de vida (TTL - Time to live)

Este campo de oito bits é usado para codificar o valor do tempo de vida do pacote, ou seja, para indicar o número de nós MPLS que um pacote atravessou para chegar ao destino. O valor é copiado do cabeçalho do pacote para o cabeçalho IP do pacote após a saída do LSP.

c) Experimental use

Este campo de três bits está reservado para uso experimental.

d) Valor do rótulo (Label Value)

Este campo de vinte bits carrega o valor do rótulo. Considerando a existência de pilhas de rótulos, um dado LSR utiliza uma tabela chamada "next hop label forwarding entry", ou NHLFE para determinar o procedimento de envio de um pacote qualquer. Na NHLFE consta qual ação deve ser realizada com a pilha de rótulos, incluindo a retirada de um rótulo do topo da pilha, inclusão de um novo rótulo no topo ou substituição do rótulo no topo da pilha e inclusão de um novo. Além dessas, consta também no NHLFE:

1) O próximo hop para enviar o pacote;

2) O encapsulamento de nível de enlace a ser usado para o envio (PPP, Frame Relay);

3) Qualquer outra informação necessária ao envio do pacote. 


\title{
3.2.7 Caminhos Comutados por Rótulos (LSP - Label Switched Paths)
}

As transmissões em redes MPLS utilizam circuitos virtuais chamados LSP (Label Switched Paths). Um LSP consiste de um caminho comutado por rótulo, ou seja, um caminho estabelecido através de uma seqüência ordenada de LSRs, entre origem e destino. Um LSP é unidirecional, portanto, é preciso a presença de dois LSPs para comunicação entre duas entidades. Uma vez que o LSP é um caminho através do qual transitarão pacotes de uma mesma classe, que compartilham o mesmo destino, uma rota deve de início ser estabelecida. Isto é feito empregando protocolos de roteamento com restrições (OSPF [35] e IS-IS [36]). Então, o caminho fica definido e os pacotes pertencentes a ele serão apenas comutados com base nos seus rótulos. Estes rótulos são distribuídos pelos LSRs no momento do estabelecimento de LSPs pelos protocolos de sinalização.

0

1

2

3

01234567890123456789012345678901

Label

| Exp|S| TTL

\author{
Label $=20$ bits \\ $\operatorname{Exp}=$ Experimental, 3 bits \\ $\mathrm{S}=$ Bottom of stack, $1 \mathrm{bit}$ \\ TTL $=$ Time to live, 8 bits
}

FIGURA 3.2: Rótulo (label) representado por 4 bytes.

Desta maneira um LSP é determinado por uma coleção de roteadores, os quais executam a comutação dos pacotes. Cada LSP possui uma série de propriedades, de maneira que para um $\mathrm{LSP}<\mathrm{R}_{1}, \ldots, \mathrm{R}_{n}>$ temos:

1) O roteador $\mathrm{R}_{1}$ corresponde ao LSR inicial do LSP, responsável por colocar o rótulo inicial nos pacotes daquele LSP; 
2) Para todo i, sendo $1<\mathrm{i}<\mathrm{n}$, todo envio de $\mathrm{R}_{i}$ para $\mathrm{R}_{i+1}$ deve seguir o procedimento MPLS. Isso implica a verificação do rótulo no topo da pilha do pacote, checagem do NHLFE para aquele rótulo e a conseqüente passagem do pacote;

\subsubsection{Rótulos de Entrada Inválidos (Invalid Incoming Labels)}

Quando um LSR recebe um pacote com um rótulo que não pertence ao grupo de rótulos de entrada válidos, não é possível verificar na NHLFE qual o próximo destino desde pacote. Uma alternativa para continuar a transmissão seria desconsiderar a pilha de rótulos e usar o cabeçalho IP para realizar o roteamento. Entretanto, se tal roteamento conduzir o pacote a algum ponto do circuito pelo qual ele já tenha passado será então formado um loop. Conseqüentemente, os pacotes têm que ser descartados.

\subsubsection{Túneis LSP}

Uma característica importante da arquitetura MPLS para proporcionar engenharia de tráfego é a capacidade de controlar a rota de um pacote sem explicitamente especificar os LSRs intermediários, criando os chamados túneis. Por exemplo, considere o LSP formado pela seqüência $<R_{1}, R_{2}, R_{3}, R_{4}>$ da Figura 3.3. Suponha que $R_{1}$ receba o pacote $P$ (IP) não rotulado e coloque em sua pilha de rótulos um rótulo enviando-o ao LSR $\mathrm{R}_{2}$. Observa-se que $R_{2}$ e $R_{3}$ não estão diretamente conectados, existindo entre eles os LSRs intermediários $R_{21}$, $\mathrm{R}_{22}, \mathrm{R}_{23}$. Pode-se assim considerar os LSRs $\mathrm{R}_{2}$ e $\mathrm{R}_{3}$, como os LSRs entre os quais existe um túnel designado pelas linhas vermelhas pontilhadas. Desta maneira a seqüência dos LSRs atravessados pelo pacote $\mathrm{P}$ é $<\mathrm{R}_{1}, \mathrm{R}_{2}, \mathrm{R}_{21}, \mathrm{R}_{22}, \mathrm{R}_{23}, \mathrm{R}_{3}, \mathrm{R}_{4}>$. Quando $\mathrm{P}$ trafega de $\mathrm{R}_{1}$ para $\mathrm{R}_{2}$, apresentará uma pilha de rótulo de profundidade 1 . O LSR $\mathrm{R}_{2}$ após receber o pacote substitui o rótulo de chegada por um outro rótulo significativo apenas para o LSR $R_{3}$, colocando também um novo rótulo. Este segundo rótulo tem um valor significativo para o LSR $\mathrm{R}_{21}$, transformando a pilha de rótulos em profundidade 2. Assim a comutação é feita através do segundo rótulo entre os LSRs $R_{21}, R_{22}, R_{23}$. O LSRs $R_{23}$ por ser o penúltimo hop, do túnel $R_{2}-R_{3}$ retira o segundo rótulo da pilha, transformando-a de novo em nível 1. O LSRs $R_{3}$ por ser o penúltimo hop do LSP 1 (linha azul-Figura 3.3), retira o rótulo colocado por $R_{2} \quad$ e $R_{4}$ recebe já o pacote sem rótulo algum, processando assim o cabeçalho IP. 


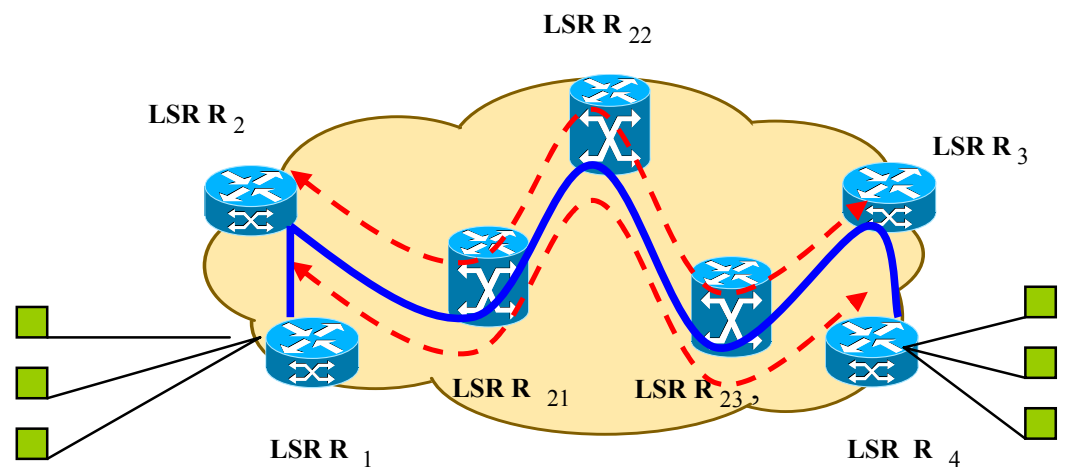

FIGURA 3.3: Exemplo de um túnel LSP formado pela seqüência $<\mathbf{R}_{2}, \mathbf{R}_{21}, \mathbf{R}_{22}, \mathbf{R}_{23}, \mathbf{R}_{3}>$ (linhas vermelhas), transportando um LSP formado pela seqüência $<\mathbf{R}_{1}, \mathbf{R}_{2}, \mathbf{R}_{3}, \mathbf{R}_{4}>$ (linha azul).

\subsubsection{Agregação}

A noção de classes de endereçamento é utilizada pelo protocolo IP através dos endereços hierárquicos, que servem para dividir os grupos de endereços nas tabelas de roteamento. A mesma noção, na tecnologia MPLS, corresponde às FECs. Contudo, a atribuição de uma FEC para cada grupo de endereçamento IP nem sempre corresponde à melhor estratégia para a criação dos circuitos MPLS, porque não é raro que grupos diferentes de endereços IP possuam a mesma interface de saída (porta de saída) em um roteador. Se forem criadas FECs diferentes em um LSR que levem ao mesmo LSR adjacente haverá uma carga de sinalização desnecessária para o estabelecimento e o controle de LSPs. Logo, foi proposto um mecanismo de "agregação" para permitir aos grupos de FECs criados, os quais possuam um destino em comum no escopo de um LSR, sejam integrados em uma única FEC. Uma estrutura de agregação pode ter vários níveis diferentes de granularidade. A granularidade mais fina corresponde a uma FEC para cada conjunto distinto de endereços IP de um LSR, e a granularidade mais espessa corresponde a ter uma única FEC para cada interface de saída, possivelmente agrupando vários conjuntos diferentes de endereços IP. A agregação pode reduzir o número de rótulos que são necessários para lidar com um conjunto particular de pacotes como também diminuir a carga de sinalização necessária ao estabelecimento e controle dos LSPs. 


\subsubsection{Roteamento Explicito e Hop by hop}

Existem duas políticas de roteamento no MPLS. Uma delas, o hop-by-hop, tem um funcionamento muito parecido com o do protocolo IP. No modelo IP o cabeçalho do pacote é analisado em todos os roteadores do percurso. No MPLS, o rótulo do pacote também é analisado em todos os LSRs do percurso, facultando a cada um deles escolher independentemente o próximo hop para uma dada FEC. A outra política de roteamento utilizada no MPLS é o roteamento explícito. Quando um pacote chega em um LSR de ingresso de um domínio MPLS, o LSR determina explicitamente o LSP, ou seja, especifica uma lista de LSRs que o pacote deverá seguir. A seqüência de LSRs componentes de um LSR explicitamente roteado pode ser escolhida por configuração manual ou ser selecionada dinamicamente pelo LSR de ingresso. Por exemplo, o LSR de ingresso pode usar as informações da topologia da rede aprendidas de uma tabela de estado de enlace confeccionada por um protocolo de roteamento (OSPF, BGP) a fim de computar o caminho seguido por um LSP. Roteamento explícito pode ser utilizado com o propósito de prover engenharia de tráfego. Para tal, as rotas necessitam ser especificadas no momento em que os rótulos são designados. A tecnologia MPLS utiliza para este fim os túneis explicitamente roteados (Explicitly Routed LSP Tunnels), sendo necessário:

1. Selecionar os pacotes, que estão para ser enviados em um túnel explicitamente roteado;

2. Configurar o túnel explicitamente roteado;

3. Assegurar que pacotes enviados não retornem ao ponto de origem caracterizando um loop;

Existem duas abordagens a serem utilizadas:

a) Começar com o protocolo RSVP, correntemente usado para configurar reservas de recursos, e estendê-lo para suportar roteamento explicito e distribuição de rótulos [24].

b) Começar com um protocolo usado para distribuição de rótulos (CR-LDP) e estendê-lo para suportar reservas de recurso e roteamento explícito [26]. 


\subsection{PRINCÍPIO DE OPERAÇÃO DO MPLS}

Nesta seção serão apresentadas as operações que devem ser executadas para que o pacote de dados trafegue através de um domínio MPLS. Para efeito de comparação, o mecanismo de roteamento IP está descrito no Apêndice II. Os pacotes originados em um LSR fonte e destinados a um determinado LSR receptor não necessariamente são transportados através do mesmo LSP. Dependendo das características de tráfego, diferentes LSPs podem ser criados. Os passos executados neste transporte são (Figura 3.4):

1. Criação e distribuição de rótulo

Antes de se iniciar o tráfego de dados entre os $\mathrm{LSRs}_{1}$ e $\mathrm{R}_{4}$, o roteador $\mathrm{LSR} \mathrm{R}_{1}$ toma a decisão de atribuir um rótulo (label) a uma FEC específica. Utilizando-se protocolos de roteamento, descobrir-se-á os enlaces através dos quais se chegará ao nó destino, iniciando-se assim um pedido de rótulo ao próximo hop, no caso LSR $\mathrm{R}_{21}$. Através de um protocolo de distribuição de rótulo (por exemplo, LDP), o pedido de rótulo será iniciado e propagado através da rede até o LSR destino, $\mathrm{R}_{4}$ (designado pela linha verde). Neste pedido as características de tráfego requisitadas serão negociadas;

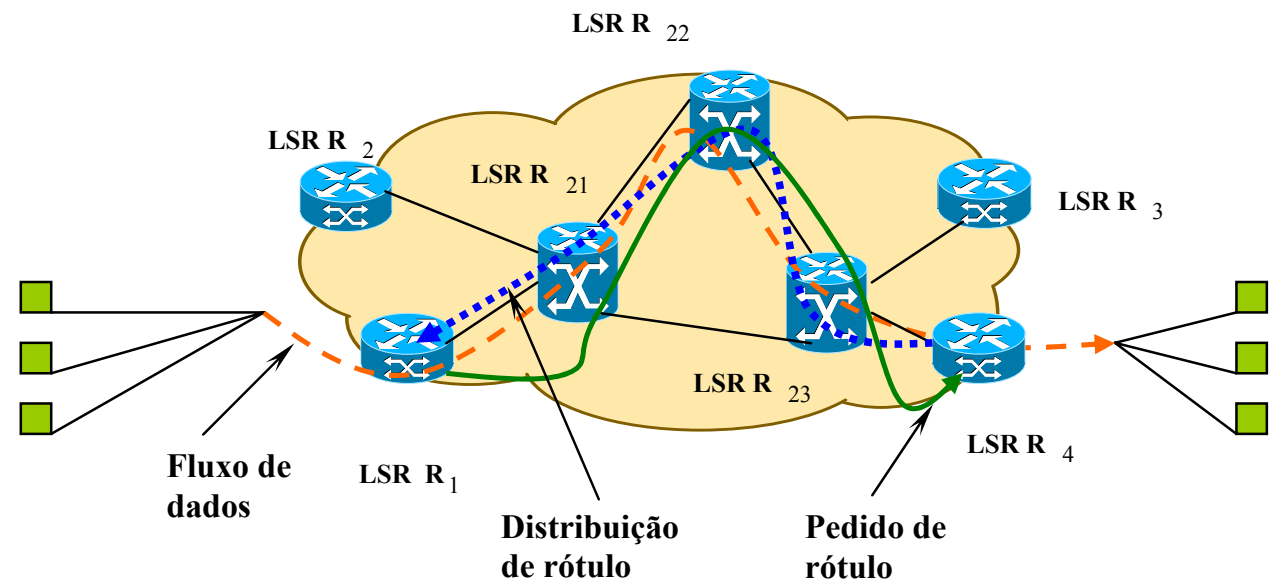

FIGURA 3.4: Fases de configuração de um LSP. O LSR $R_{1}$ é especificado como o LSR upstream e o LSR $\mathbf{R}_{4}$ como dowstream. 
2. Criação de tabela

Cada LSR intermediário receberá o rótulo designado a uma FEC de seu LSR downstream, começando do LSR $\mathrm{R}_{23}$ e retrocedendo até o LSR $\mathrm{R}_{1}$ (designado pelo linha azul). Ao receber o rótulo, cada LSR cria uma entrada na tabela NHLFE (Next Hop Label Forwarding Entry). Como visto anteriormente, o conteúdo desta tabela especificará o mapeamento entre um rótulo e uma $\mathrm{FEC}$, determinando assim o procedimento de envio de um determinado pacote. Desta maneira está configurado o LSP;

3. Envio do pacote

$\mathrm{O}$ LSR $\mathrm{R}_{1}$ inserirá o rótulo já designado e enviará o pacote ao LSR $\mathrm{R}_{21}$. Cada LSR subseqüente, isto é, os LSRs $R_{22}$ e $R_{23}$ examinará o rótulo do pacote recebido, substituindo-o pelo respectivo rótulo de saída e o enviará ao próximo hop. Quando o pacote alcança o LSR $\mathrm{R}_{4}$ este removerá o rótulo (o rótulo pode também ser removido pelo LSR anterior (LSR $\mathrm{R}_{23}$ ) se uma função denominada penultimate hop popping estiver sendo utilizada). O fluxo de dados seguido pelo pacote é designado assim pela linha vermelha mostrada na Figura 3.4.

\subsection{ESTRUTURAS DE REDES MPLS}

Como visto anteriormente redes utilizando MPLS (Figura 3.5) consistem de LSRs de borda (edge label switch routers - LSRs) e LSRs de núcleo (core LSR). A Figura 3.5 mostra quatro LSRs de borda e nove equipamentos de consumidores. Entretanto, existirão centenas destes por LSR de borda. Os equipamentos dos clientes conectados em uma rede MPLS tipicamente empregam o protocolo IP em vez de MPLS, e são roteadores ou switches LAN. Uma vez que tais equipamentos não utilizam MPLS, os LSRs de borda podem pertencer à rede de um provedor de serviço e estarem sob administração deste.

\subsubsection{Protocolos em redes IP e MPLS}

Redes MPLS utilizam um conjunto de protocolos empregados também em roteadores IP, tornando o MPLS e o IP completamente interoperáveis. A arquitetura IP, ou pilha IP, consiste de 
um grande número de protocolos, um subconjunto dos quais utilizados nos roteadores IP. A Figura 3.6 (a) mostra os mais importantes destes protocolos como base para comparação com a pilha MPLS.

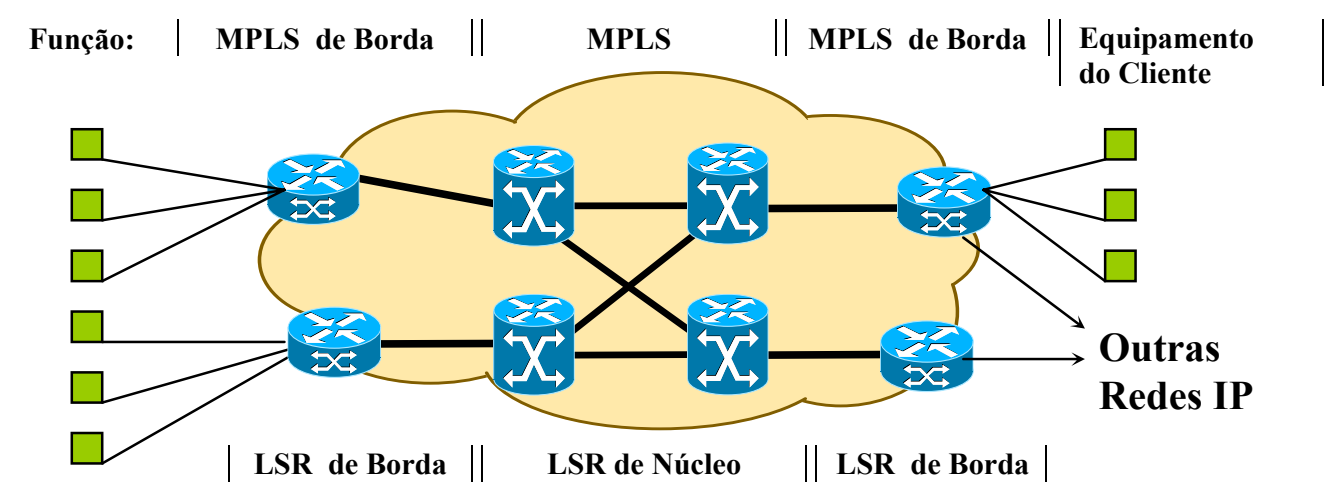

FIGURA 3.5: Arquitetura geral de uma rede MPLS.

Os protocolos presentes em um roteador IP consistem de protocolos de roteamento e de envio. Protocolos de roteamento informam o roteador sobre as rotas para alcançar o desejado destino IP. Cada roteador estará, em geral, utilizando um IGP (interior gateway protocol), o qual lida com o roteamento dentro da rede. Em outras palavras, IGPs são protocolos de roteamento utilizados dentro de um sistema autônomo (autonomous system - AS) para prover roteamento intra-AS. A expressão sistemas autônomos (ASs) tem a intenção de designar sub-redes. Existe uma escolha de padrões de IGPs:

\section{RIP (Routing Information Protocol)}

Era o IGP original da Internet. Protocolo de vetor distância baseado no algoritmo de BellmanFord [2], funcionava bem em sistemas pequenos com poucos ASs conectados, apresentando queda de performance quando o tamanho e complexidade das redes aumentavam. Protocolos de vetor distância operam fazendo com que cada roteador mantenha uma tabela (ou seja, um vetor) que fornece a distância mais otimizada para cada destino e determina qual rota deve ser utilizada para se chegar a este ponto. Tal protocolo apresentava uma convergência lenta, tornando-o, nos dias atuais, obsoleto. 


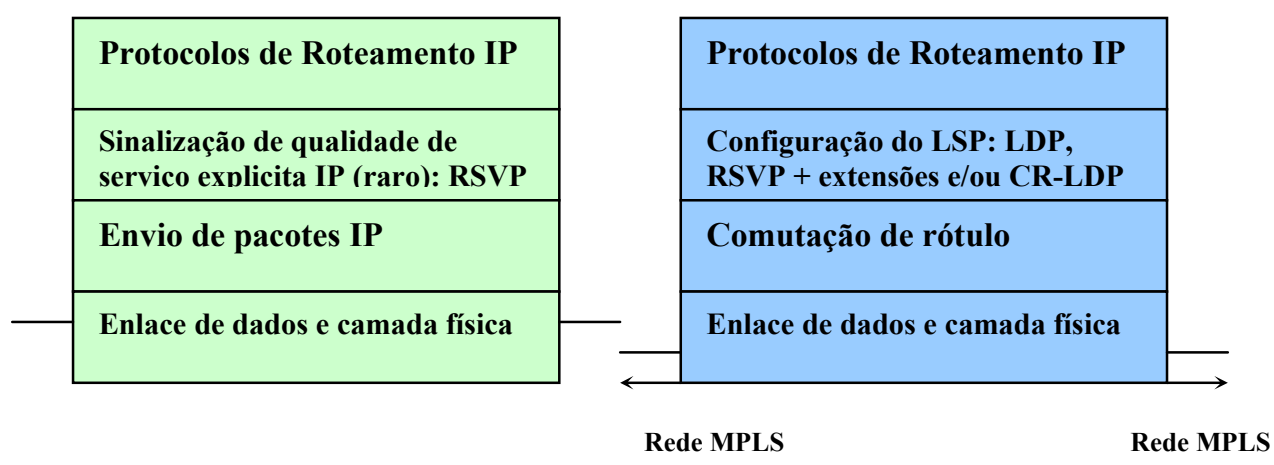

a) Roteador IP

\begin{tabular}{|c|c|c|}
\hline Protocolos de Roteamento IP & & Protocolos de Roteamento IP \\
\hline $\begin{array}{l}\text { Configuração do LSP: LDP, } \\
\text { RSVP + extensões e/ou CR-LDP }\end{array}$ & & $\begin{array}{l}\text { Sinalização de qualidade de } \\
\text { servico explicita IP (raro): RSVP }\end{array}$ \\
\hline Comutação de rótulo & $\begin{array}{l}\text { Encapsulamento } \\
\text { de pacotes MPLS }\end{array}$ & Envio de pacotes IP \\
\hline Enlace de dados e camada física & & Enlace de dados e camada física \\
\hline
\end{tabular}

Rede MPLS b) LSR

Rede IP

\section{c) LSR conectado a um roteador IP- LSR de borda}

CR-LDP: Constraint-based routing with LDP

LDP: Label Distribution Protocol
MPLS: Multiprotocol label switching

LSR: Labl switch router

RSVP: Resource Reservation Protocol

FIGURA 3.6: Pilhas simplificadas de Protocolos para IP e MPLS [28]

\section{OSPF (Open Shortest Path First)}

Padronizado em 1990 pelo IETF o protocolo OSPF (atualmente designado pela RFC 2328) utiliza o algoritmo de roteamento por estado de enlace. As funções executadas por roteadores utilizando este algoritmo são: descoberta de roteadores presentes em sua sub-rede e seus respectivos endereços de redes, medição do retardo ou custo para atingir cada um destes roteadores, criação e envio de pacotes a estes roteadores nos quais se especifica as informações aprendidas sobre a topologia da rede, cálculo do caminho mais curto a ser utilizado para alcançar cada um destes roteadores. Fundamentalmente o protocolo OSPF representa a rede real como um grafo e, em seguida, calcula o caminho mais curto de um roteador a outro roteador. É muito utilizado, embora o protocolo IS-IS (Interior System to Interior System) seja também comum entre grandes ISP e concessionárias de telecomunicações. 
Tipicamente, todos roteadores sob uma dada área administrativa como, por exemplo, um sistema autônomo (AS), empregam o mesmo IGP. Em geral, um roteador utilizado para a comunicação entre diferentes administrações (ASs) empregará outro protocolo de roteamento chamado de BGP (Border Gateway Protocol). Este protocolo torna-se necessário pois os objetivos de um protocolo de gateway interno e os de um protocolo de gateway externo não são os mesmos. Tudo que o primeiro deve fazer é movimentar pacotes da forma mais eficiente possível, da origem ao destino, não importando a política de tráfego. Já os roteadores de protocolo de gateway externo têm esta preocupação. Por exemplo, um AS corporativo possui a capacidade de enviar e receber pacotes para qualquer site da Internet. Entretanto, talvez este AS não deseje transportar pacotes originados em um AS externo com destino a outro AS externo, mesmo que seu próprio AS esteja no caminho mais curto entre estes dois ASs externos.

Por outro lado, talvez seja desejável transportar pacotes para seus vizinhos ou mesmo para outros ASs específicos, os quais tenham pago pelo serviço. Tais políticas são manualmente configuradas em cada roteador BGP, não fazendo parte do protocolo em si. Cada roteador BGP contém módulos, os quais examinam e computam as rotas para um determinado destino, retornando um número para identificar a distância até este destino em relação a cada rota. Qualquer rota cujo tráfego através dela viole uma restrição da política utilizada recebe automaticamente uma contagem infinita. Em seguida, o roteador adota a rota com a distância mais curta. Atualmente este protocolo está definido na recomendação IETF RFC 1771.

Protocolos de roteamento automaticamente constroem uma tabela de informações de envio para e em cada roteador. A tabela de envio lista os prefixos de destinos IP conhecidos e a referência em qual enlace de dados no roteador está o próximo hop quando este necessitar mover os pacotes IP para tais destinos. A versão 4 (IPv4) é a mais utilizada para aplicações IP, embora o uso da versão 6 (IPv6) esteja sendo introduzida.

Muitos outros protocolos são usados por roteadores. Existem protocolos específicos para vários meios e protocolos para executar funções auxiliares. Por exemplo, protocolos de roteamento usam o TCP (Transport Control Protocol) ou o UDP (Universal Datagram Protocol) como parte do processo de comunicação. Entretanto, estes protocolos não são usados nas principais funções dos roteadores, isto é, no envio de pacotes ao longo da rota correta para o destino desejado.

A recomendação IETF RFC 3000 "Internet Official Protocol Standards", (possíveis atualizações desta RFC serão designadas pelos números 3100, 3200, etc) apresenta uma 
completa lista de todos os protocolos da pilha IP, status de padronização e referências para as respectivas RFCs que os descrevem .

\subsubsection{Protocolos no núcleo da rede MPLS}

Como um roteador comum, o LSR de núcleo utilizará um ou mais protocolos de roteamento IP (Figura 3.6 b). Entretanto, ao invés do envio de pacotes IP, os LSRs usam, como descrito anteriormente, um tipo de mecanismo diferente de envio denominado LSP (label switching path). Um LSP, no caso mais simples, corresponde a um prefixo de destinação IP tal qual em tabelas de envio de alguns roteadores. Por exemplo, o IGP em uma sucessão de LSRs poderia criar entradas na tabela de envio para um prefixo de destino IP 10.1.1.0/24. Os LSRs, com efeito, comparam as informações de envio para determinar que todos possuem uma rota para 10.1.1.0/24 e configuram um LSP através deles a fim de carregar todo o tráfego para o prefixo de destino IP 10.1.1.0/24. A configuração de um LSP de acordo com informações de roteamento IP é conhecida como roteamento MPLS hop-by-hop. O LSP também pode ser roteado, como mencionado anteriormente, de uma maneira explicita, ou seja, a rota é estabelecida no ingresso do pacote na rede não sendo facultado aos LSRs de núcleo escolher independentemente o próximo hop.

A configuração de LSPs é feita, como anteriormente mencionado, por um processo de distribuição de rótulo. Existem algumas combinações válidas de protocolos para a distribuição de rótulo:

1. Em geral, todas as redes MPLS usam o LDP ( label distribution protocol), o qual suporta o MPLS roteado hop-by-hop;

2. Uma alternativa para rotear explicitamente o MPLS é uma combinação do LDP com algumas extensões, chamadas CR-LDP (constraint-based routing with LDP);

3. Outra alternativa para rotear explicitamente o MPLS é uma combinação de RSVP com algumas extensões;

A seguir, descrever-se-á algumas características destes protocolos de sinalização: 


\subsubsection{Características do Protocolo de distribuição de Rótulos}

A arquitetura MPLS define um protocolo de distribuição de rótulos como um conjunto de procedimentos pelo qual um LSR informa a outro o significado dos rótulos usados para envio de tráfego entre e através deles. Tal esquema não assume um protocolo de distribuição de rótulos específico. De fato, diferentes protocolos de distribuição de rótulos estão sendo padronizados. Como visto anteriormente, os protocolos existentes têm sido estendidos para realizar a distribuição de rótulos. Novos protocolos também têm sido definidos para propósitos de distribuição de rótulos.

O protocolo de distribuição de rótulos (LDP - Label Distribution Protocol) [25], define um conjunto de procedimentos e mensagens pelas quais os LSRs estabelecem LSPs através de uma rede, mapeando informações de roteamento da camada de rede para a camada de enlace (colocação de rótulo). Esses LSPs podem ter seu ponto final em um nó vizinho (envio hop-byhop) ou em um nó de saída da rede, habilitando a comutação via todos os nós intermediários.

O LDP associa assim uma FEC a cada LSP criado nos LSRs de ingresso da rede, especificando quais pacotes são mapeados para qual LSP. Tais LSP são estendidos através da rede quando cada LSR conecta rótulos de chegada para uma FEC a rótulos de saída designados para o próximo nó. Desta maneira dois LSRs, os quais usam o LDP para trocar informações de atribuição de uma FEC a um determinado rótulo são conhecidos como LDP pares e estabelecem uma sessão entre eles. Os LDPs usam o protocolo TCP para esta comunicação. Tal uso assegura a troca de mensagens de forma confiável, de maneira que as informações de estado e distribuição de rótulos não necessitam serem renovadas periodicamente [29].

\subsubsection{Características do CR-LDP (constraint-based routing with LDP)}

Um dos mais importantes serviços oferecidos por redes MPLS em geral e pelo CR-LDP em particular é o suporte ao roteamento de tráfego baseado em restrições. Tal abordagem oferece a oportunidade de estender a informação usada para configurar um caminho além daquelas disponíveis para o protocolo de roteamento. Por exemplo, um LSP pode ser configurado baseado em restrições de rota explícita (utilização somente de LSRs predeterminados), restrições de QoS e outras. Tal LSP é denominado CR-LSP (constraint-based label switched paths). Em outras palavras, o CR-LSP é um LSP cuja configuração é baseada em critérios, incluindo, mas não limitados, às informações de roteamento. O intento é agregar características especiais ao LSP a 
fim de melhor suportar o tráfego enviado. A razão para configurar CR-LSPs está ancorada no desejo de designar certas restrições como largura de banda e outras classes de serviço para o LSP, ou certificar-se de que rotas alternativas usem caminhos fisicamente separados através da rede [26]. O protocolo CR-LDP é assim definido com o propósito de estabelecer e manter LSPs roteados explicitamente [30]. A configuração de um LSP utilizando CR-LDP é apresentada no Apêndice II.

O protocolo de sinalização CR-LDP pode prover, entre outras capacidades, a configuração de rotas explicitamente roteadas através de alocação de roteadores individuais (strict explicit routes) ou grupos de roteadores (loose explicit route) e realocação de recursos para os caminhos através de prioridades (path preemption with setup and holding priority). Este mecanismo permite ao CR-LDP realocar recursos requeridos por uma determinada rota. Se uma rota com recursos suficientes não pode ser encontrada, caminhos existentes podem ser reconfigurados para realocar recursos para o novo caminho, processo este denominado de path preemption. Prioridade de configuração (setup priority) e designação de prioridades (holding priority) são usados para ordenar caminhos existentes (holding priority) e novos caminhos (setup priority), no intuito de determinar se a nova rota pode dispor dos recursos de uma rota existente. Setup priority de um novo caminho e holding priority de um caminho já existente são usados para especificar prioridades. Sinalizar uma alta holding priority expressa que o caminho, uma vez estabelecido, possui uma chance menor de ter seus recursos utilizados por outro caminho. Sinalizar uma alta setup priority expressa que em caso de não disponibilidade imediata de recursos, a solicitação terá a maior chance de obtê-los. Tais valores de prioridade para o caminho fazem parte da política da rede. A alocação de valores de prioridade é feita de zero (0) a sete (7). O valor zero (0) é designado para o caminho mais importante, sendo referido como alta prioridade. $\mathrm{O}$ valor sete (7) é referido como baixa prioridade. $\mathrm{O}$ uso de valores de prioridade padrão é um aspecto da política da rede, sendo recomendado o valor quatro (4).

\subsubsection{Características do RSVP}

O protocolo de sinalização RSVP foi originalmente concebido para realizar reserva de recursos em redes utilizando protocolos IP, com o intuito de oferecer serviços integrados (IntServ). A arquitetura IntServ visa fornecer, em uma rede comutada por pacotes, como a Internet, a emulação mais próxima possível de circuitos virtuais. A idéia principal subjacente ao IntServ é a de reserva de recursos. Antes de iniciar a transmissão dos dados, as aplicações 
precisam encontrar uma rota até o receptor que satisfaça suas demandas de QoS, reservando, ao longo do mesmo, os recursos necessários. Desta maneira o RSVP clássico (IETF RFC 2205) assegura aos roteadores a flexibilidade para preservar o comportamento de transporte sem conexão enquanto implementam reservas de recurso.

Para servir como protocolo de distribuição de rótulos para LSPs com restrições em redes MPLS, as mensagens do protocolo RSVP foram ampliadas com novos objetos (isto é, padrões de sinalização utilizado por este protocolo). Em se tratando do protocolo CR-LDP o padrão será o TLV (Time-Length-Value) que permite suportar tanto alocação de rótulos, distribuição, e anexação destes a um FEC, quanto roteamento explícito. Tais extensões incluem suporte a realocação de recursos para os caminhos através de prioridades (path preemption with setup and holding priority), detecção de loop e definição de rotas explicitamente roteadas através da alocação de roteadores individuais ou grupos de roteadores para a rota desejada (strict and loose explicit routes) [31].

\subsubsection{Resumo das funções dos Protocolos CR-LDP e RSVP-TE}

Os protocolos de sinalização CR-LDP e RSVP-TE executam funções similares em redes MPLS. Ambos buscam de uma maneira ou outra suprir os seguintes requisitos:

1. Robustez: O protocolo de sinalização deve ser capaz de assegurar confiabilidade e entrega oportuna de mensagem de sinalização mesmo em presença de congestionamento ou falhas na rede;

2. Escalabilidade: $\mathrm{O}$ tamanho das redes requer o suporte para um grande número de LSPs em cada nó. O sistema de sinalização deve ser escalável para que possa continuar a proporcionar a mesma performance caso o número de nós e caminhos cresça com a rede;

3. Especificação de QoS: Inclui a especificação de descritores de tráfego (i.e. requerimento de largura de banda) associado com o fluxo de tráfego usando um LSP, e o requerimento de QoS (por exemplo retardo e perda);

4. Estabelecer, manter e encerrar LSPs: Isto requer eficiente gerenciamento do estado dos LSP e a habilidade para mudar os seus parâmetros;

5. Proporcionar realocação e prioridades aos LSPs (priority/preemption): Realocação de caminhos é o requerimento de engenharia de tráfego para assegurar que um LSP de alta prioridade possa se apoderar de recursos de um LSP de baixa prioridade quando não 
existem recursos suficientes para ambos. Sinalizar uma alta designação de prioridade (priority holding) assegura a um LSP uma chance pequena de ser realocado;

6. Flexibilidade em opções para configurar rotas: Inclui CR-LSPs restritos e indefinidos (strict/loose). Estas capacidades permitem ao operador de rede especificar o caminho completo (nós e interfaces) que um LSP atravessa (strict CR-LSPs), ou solicitar que uma porção da rota seja configurada automaticamente com base no protocolo de roteamento (loose CR-LSPs);

7. Configuração de caminhos alternativos e reroteamento: otimização de caminhos e recuperação de falhas são essenciais para que as redes proporcionem serviços seguros. Situações de falhas devem ser identificadas e resolvidas prontamente, com um mínimo de mensagens de controle e processamento de overhead;

Não existe consenso sobre qual protocolo é tecnicamente superior. Entretanto, administradores de rede devem fazer a escolha entre os dois, com base em suas necessidades e situações particulares (IETF RFC 3213). Uma comparação pormenorizada entre tais protocolos pode ser encontrada em [32] e no Apêndice II.

\subsubsection{Protocolos em dispositivos de borda em redes MPLS}

LSRs de borda conectam redes MPLS às tradicionais redes IP. Em conseqüência, empregam todos os protocolos encontrados em roteadores IP, incluindo o envio de pacotes IP em adição à comutação de rótulos empregada pelo MPLS, como ilustrado na Figura 3.6 (2c).

Protocolos de roteamento IP não fazem distinção entre redes MPLS e IP. Para os propósitos de envio de pacotes, as pilhas de protocolos do IP tradicional e do MPLS em LSRs de borda são ligadas pelo processo de encapsulação MPLS.

Em operação simples, a encapsulação MPLS envolve a aplicação ( pushing) de um rótulo em um pacote IP, o qual é então enviado através da rede no LSP. Quando o pacote alcança a borda da rede MPLS, o rótulo é então removido ( popped) do pacote, o qual é enviado como um pacote IP ordinário.

A arquitetura MPLS também permite, como visto anteriormente, que mais de um rótulo seja carregado em cada pacote. Assim, uma pilha de rótulos de tamanho arbitrário pode ser carregada usando um cabeçalho de rótulo MPLS. Múltiplos rótulos são usados em aplicações como, por exemplo, engenharia de tráfego. 


\subsection{ENGENHARIA DE TRÁFEGO}

O termo Engenharia de Tráfego abrange vários aspectos relacionados ao desempenho de uma rede. Esta denominação inclui provisão de QoS, melhoria na utilização dos recursos da rede pela reordenação uniforme do tráfego através desta e provimento de funcionalidade para rápida recuperação em caso de falha de um nó ou enlace.

Existem quatro componentes básicos necessários à engenharia de tráfego em redes de pacotes [33]:

1. Distribuição de informação de topologia: como a rede é um sistema distribuído, torna-se necessário dispor de um mecanismo para propagação de informações sobre os enlaces disponíveis para todos os nós. Um nó pode então construir um mapa da topologia da rede. Informações sobre falhas em nós devem ser propagadas através da rede, iniciandose assim uma rápida recuperação;

2. Seleção de rota: envolve utilizar as informações reunidas sobre a topologia para selecionar um caminho entre nós em uma rede. Quando um caminho é solicitado entre qualquer par de nós em uma rede, o caminho mais curto com um mínimo de enlaces é tipicamente escolhido, pois isto otimiza o uso de recursos disponíveis. Outras restrições, tais como largura de banda e retardo máximo, podem também ser aplicadas;

3. Direcionamento de tráfego ao longo do caminho computado: uma vez que o caminho entre um dado nó fonte e um nó de destino tenha sido computado, deve-se ser capaz de direcionar o tráfego ao longo deste caminho particular. Tipicamente, isto é acompanhado pela construção de uma tabela de envio. Quando um pacote atinge um nó, a tabela de envio é consultada usando informações presentes no cabeçalho do pacote. Em uma rede com envio de pacotes sem conexão, cada nó constrói sua tabela de envio independentemente. Para as redes com envio orientado a conexão, este passo envolve a utilização de um protocolo de sinalização para configurar o caminho. Tal protocolo auxilia os nós intermediários ao longo do caminho a construírem suas tabelas de envio; 
4. Gerenciamento de tráfego: $\mathrm{O}$ gerenciamento de tráfego trata dos mecanismos que proporcionam QoS aos usuários deste tráfego. A caracterização de QoS é feita por parâmetros tais como: atrasos, jitter e probabilidade de perdas. Os mecanismos para implementar QoS incluem controle de admissão, identificação de fluxo e políticas de tráfego;

Torna-se patente a simbiose entre os conceitos básicos de ambos, engenharia de tráfego e MPLS. Em outras palavras, a aplicação de conceitos básicos do MPLS para propiciar engenharia de tráfego às redes IP, por exemplo, pela utilização de LSP roteados explicitamente, atenuando a sub utilização de recursos resultante das decisões individuais de cada roteador, utilizando sempre a rota de menor caminho. No restante desta seção serão descritos os objetivos da engenharia de tráfego e como o MPLS pode ser utilizado na sua implementação.

\subsubsection{Objetivos da Engenharia de Tráfego}

Uma função da engenharia de tráfego em redes IP é o mapeamento do tráfego na infraestrutura da rede. Alta qualidade de serviço, eficiência e economia são objetivos cruciais a serem atingidos pelos provedores de serviços (ISPs) na Internet competitiva de hoje.

Um requerimento essencial para engenharia de tráfego em redes IP é a capacidade de comutar e estabelecer rotas de envio entre um nó a outro. Esta rota deve preencher alguns requisitos e políticas de restrições na rede. Geralmente os objetivos podem ser orientados ao tráfego (traffic-oriented) ou orientado aos recursos (resource-oriented).

$\mathrm{O}$ primeiro diz respeito à melhoria do QoS oferecida pela Internet, incluindo variáveis como perda de pacote, atraso e variação do atraso (jitter). Objetivos orientados aos recursos relacionam-se à otimização da utilização da rede [34].

Minimizar congestionamentos é a meta principal da engenharia de tráfego. Tais congestionamentos tipicamente manifestam-se nos seguintes cenários:

1. Quando recursos de rede são insuficientes ou inadequados para lidar com a carga de tráfego oferecida;

2. Quando o tráfego é ineficientemente mapeado aos recursos existentes, fazendo que subconjuntos de recursos sejam super utilizados enquanto outros permanecem sub utilizados; 
Problemas de congestionamento resultam da insuficiência e inadequação dos recursos e podem ser resolvidos pelo aumento da capacidade da rede ou controle da demanda para que o tráfego adapte-se à capacidade disponível, utilizando-se, por exemplo, de políticas de controle de fluxo, tarifas e outras medidas.

Tornar as redes confiáveis é outro objetivo importante da engenharia de tráfego. Cenários de recuperação de falhas devem ser planejados para assegurar a continuidade dos serviços após a deterioração da rede e a capacidade para restauração de serviços deve estar disponível. De fato, deve-se contar com a capacidade operacional de eficientemente reorientar o tráfego através de recursos redundantes quando falhas ocorrerem.

\subsubsection{Aplicando o MPLS para Engenharia de Tráfego}

A aplicação do MPLS em engenharia de tráfego faz uso de mecanismos para conduzir pacotes através da rede (túneis LSP), de meios para encontrar caminhos apropriados através da rede (distribuição de informação), bem como de formas para direcionar o tráfego através de LSPs e responder às mudanças de topologias. Estes aspectos resultam da aplicação dos conceitos básicos do MPLS vistos anteriormente, sendo formados pelos seguintes componentes:

a) Gerenciamento de caminho;

b) Designação de tráfego;

c) Gerenciamento de rede;

d) Disseminação de informações do estado da rede;

\subsubsection{Gerenciamento de caminho}

O gerenciamento de caminho diz respeito a todos os aspectos relacionados à seleção de rotas explícitas e a manutenção de LSPs túnel. Este termo deriva do fato de que pacotes trafegando ao longo destes túneis estão temporariamente tunelados abaixo do mecanismo normal de roteamento IP. Uma política de gerenciamento de caminhos define os critérios e regras para sustentar LSP já estabelecidos. O gerenciamento de caminho consiste de três funções: seleção de caminho, disposição de caminho e manutenção de caminho. 
A função de seleção de caminho especifica rotas explicitas para um LSP túnel no nó de origem deste. Uma rota, como anteriormente já mencionado, pode ser representada como uma seqüência restrita ou indefinida de nós (strict and losse subsets). Rotas explícitas podem ser definidas administrativamente ou computadas automaticamente por uma entidade de roteamento baseada em restrições (constraint-based routing entity). Esta entidade é usada para computar rotas, que satisfaçam a um conjunto de requerimentos, subordinados às restrições impostas pela rede ou por políticas administrativas. Detalhes da função de roteamento baseada em restrições pode ser encontrados em [34].

A função de disposição de caminho é usada para inicializar um LSP túnel usando um protocolo de sinalização (CR-LDP e RSVP), os quais servem como protocolos para distribuição de rótulos. Um importante requerimento operacional é a capacidade de manipular os atributos de um LSP túnel ativo para causar certas transições (mudanças de rotas explícitas) sem impactar a operação da rede. Atributos de LSP túneis incluem, atributos de alocação de recursos (preemption attributes), parâmetros de tráfego ( taxa de pico, largura da banda).

\subsubsection{Designação de tráfego}

Designação de tráfego diz respeito a todos os aspectos relacionados à alocação de tráfego para LSP túneis estabelecidos. Em outras palavras, dada uma certa necessidade de tráfego, como encontrar o LSP túnel que atenda tal exigência?

Uma solução geral consiste na utilização de duas funções, uma função de partição e outra de distribuição. A primeira, particiona o tráfego entrante de acordo com algum princípio de divisão, distribuído pela segunda função aos LSP túneis respeitando-se alguma forma de alocação. Um modo de se realizar tal designação é fazer com que, de posse das necessidades de tráfego, o nó calcule o caminho que satisfaça os requisitos de transporte em uma lista de LSP túneis, dentro de um domínio IGP.

A distribuição de carga através de múltiplos LSP túneis entre dois nós é uma importante questão em designação de tráfego. O problema de distribuição de carga pode ser abordado designando pesos a cada túnel LSP e distribuindo-se o tráfego de forma proporcional a tal peso. 


\subsubsection{Gerenciamento de rede}

De forma geral, um sistema de gerenciamento de redes MPLS inclui um conjunto de funções de gerenciamento de configuração, de desempenho de falhas. Coletivamente, estas funções permitem determinar o estado dos objetos MPLS gerenciados (ex. LSP túneis), controlando assim suas características. Como exemplo, fluxos de tráfego podem ser caracterizados pelo monitoramento das estatísticas de tráfego em LSP túneis. Perdas de pacote podem ser estimadas, monitorando-se as estatísticas de tráfego de entrada e saída nos nós finais de um LSP (uma vez que túneis LSP são unidirecionais). Características de atrasos de caminho podem ser estimadas, enviando-se pacotes de testes através dos LSP túneis e medindo-se o tempo de trânsito.

Um requerimento operacional importante é a capacidade de listar, a qualquer momento, todos os nós atravessados por um LSP túnel e para cada nó, listar todos os LSP túneis que o atravessam.

\subsubsection{Disseminação de informações do estado da rede}

Embora o MPLS ofereça um método para configuração de caminhos e envio de tráfego, a questão de como encontrar caminhos com restrições não é abordada. Para habilitar a determinação de rotas com restrições, o IETF tem como objetivo estender os IGPs já utilizados (OSPF [35] e IS-IS [36]) para transportarem informações adicionais sobre os enlaces. Tais informações incluem largura de banda máxima, bem como a prioridade para realização de reserva de recursos, baseada em oito níveis de prioridades e métricas relacionadas à engenharia de tráfego.

Tais métricas tornam os processos de roteamento não mais restritos à métrica geralmente usada pelos IGPs, relacionadas ao caminho mais curto. A informação do estado da topologia é usada para selecionar possíveis rotas para os LSP túneis.

De uma maneira geral o IETF procurou estender não só os protocolos IGPs para trabalharem com restrições de rotas em redes MPLS, como também a própria topologia MPLS para trabalhar como plano de controle em redes ópticas. Este assunto será abordado no Capítulo 4 . 


\subsubsection{Limitações da Aplicação de MPLS para prover Engenharia de Tráfego}

A implementação de engenharia de tráfego utilizando MPLS (MPLS-TE- Traffic Engineering) é uma técnica nova quando comparada aos protocolos de roteamento IP clássicos (OSPF, BGP). Por este motivo recursos significativos em treinamento de recursos humanos são necessários para compreender como a tecnologia MPLS trabalha, especialmente na implementação de redes com equipamentos de diferentes fabricantes. Problemas de escalabilidade podem surgir se o número de LSPs aumenta. A perda de escalabilidade pode ocorrer via instabilidade dos novos protocolos e suas extensões (CR-LDP e extensões para OSPF) ou inabilidade em mudar a configuração da rede para acomodar o crescimento desta.

Muitos equipamentos instalados em redes de provedores de serviço estão já em estado de sobrecarga. Habilitar o MPLS-TE em uma rede envolve adicionar novos protocolos e novas informações a serem carregadas pelos protocolos de roteamento. Em alguns ambientes de rede, esta carga adicional pode ser excessiva.

Ademais, o MPLS e em geral o MPLS-TE, não são uma panacéia para a falta de capacidade dos recursos de uma rede em proporcionar um meio adequado de transporte a fluxos de dados ou para a falta de planejamento no dimensionamento correto desta. Nestas condições, o MPLS-TE pode levar o tráfego de uma rede a atravessar distâncias maiores ou uma quantidade maior de elementos de redes (roteadores). Falhas no planejamento e implementação de uma rede levarão a congestionamentos, não importa qual tecnologia seja empregada [37]. 


\section{CAPÍTULO 4}

\section{ARQUITETURA MPLS GENERALIZADA - GENERALIZED MPLS}

Neste capítulo serão apresentadas as extensões necessárias à arquitetura MPLS para dar suporte a utilização de dispositivos, ou melhor, interfaces, nas quais a decisão de envio é baseada em intervalos de tempo (time slots), comprimentos de onda ou portas físicas, dando origem à arquitetura Generalized MPLS (GMPLS). Finalizando, serão descritos os dois modelos de interconexão (coberto - overlay e de pares - peer) suportados por esta nova arquitetura.

\subsection{INTRODUÇÃO}

Um dos pontos principais em uma arquitetura de rede é o seu plano de controle. A função principal do plano de controle é configurar conexões dinamicamente. Ou seja, redes ópticas formadas por OXCs e OADMs, utilizar-se-ão de um plano de controle comum para dinamicamente alocar recursos e prover operação livre de falhas, empregando técnicas de proteção e restauração. A Figura 4.1 apresenta a diferença entre redes com gerenciamento centralizado e distribuído onde se aplica o conceito de plano de controle. Neste caso, o objetivo é configurar conexões via sinalização distribuída de uma maneira mais rápida do que a disponibilizada por redes com gerenciamento centralizado. Através da definição de interfaces de sinalização (UNI e NNI), a operação manual executada por operadores de redes torna-se desnecessária e conexões podem ser configuradas automaticamente de uma forma mais rápida. A despeito das diferenças existentes nas tecnologias e formas de operação das diferentes redes, especialmente no plano de dados, todas as redes apresentam características similares em seus planos de controle. 


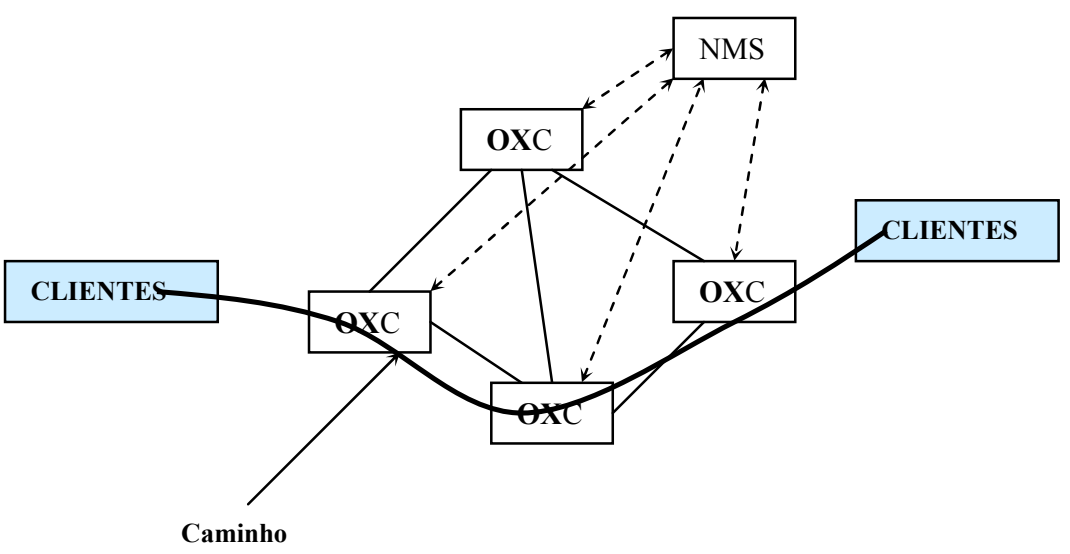

NMS: network management system

a) Rede baseada em plano de gerenciamento centralizada

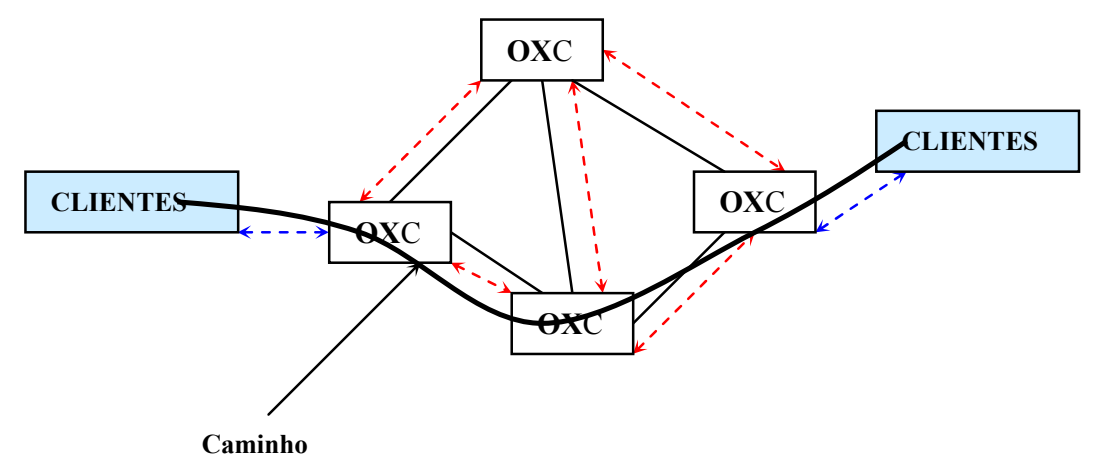

UNI: user network interface

Interface entre o cliente e a rede

$\leftrightarrow-\rightarrow \quad$ NNI: network node interface

Interface entre os nós da rede

b) Rede baseada em plano de controle distribuído utilizando interfaces UNI e NNI

FIGURA 4.1: Formas de configurar conexões em: a) Redes com gerenciamento centralizado e b) Redes com gerenciamento distribuído. 
A necessidade de sustentar múltiplos tipos de tráfego e camadas (IP, ATM, SONET-SDH) e proporcionar um suporte à interoperabilidade de equipamento de diferentes fabricantes, direcionam o interesse em definir um plano de controle único, MPLS generalizado (generalized MPLS).

A tecnologia MPLS tem a capacidade de separar o plano de controle do plano de envio, ou seja, a utilização de rótulos permite múltiplas formas de roteamento no plano de controle (ex. rotas explícitas, hop-by-hop). De fato, a arquitetura MPLS foi definida para suportar o envio de dados baseados em rótulos anexados a pacotes. Nesta arquitetura, os LSRs apresentam um plano de envio capaz de reconhecer pacotes ou células e transportá-los através da rede. A arquitetura MPLS original está, assim, sendo estendida para incluir equipamentos cujos planos de envio não reconhecem apenas pacotes. Especificamente, estes novos equipamentos incluem dispositivos onde a decisão de envio é baseada em intervalos de tempo, comprimentos de onda ou portas físicas (OXCs). Sendo assim, o novo conjunto de dispositivos, ou, mais precisamente, interfaces nestes dispositivos, podem ser subdivididos nas seguintes classes [38]:

1. Interfaces capazes de comutar pacotes - PSC (Packet Switch Capable): Interfaces que reconhecem pacotes e podem enviar dados com base no conteúdo do cabeçalho do referido pacote. Como exemplo, pode se citar as interfaces em roteadores IP;

2. Interfaces capazes de comutar com base na camada 2 - L2SC (Layer-2 Switch Capable) Interfaces que reconhecem quadros (frames) ou células, e podem enviar dados com base no conteúdo destas. Como exemplo, pode se mencionar as interfaces ATM em LSRs que enviam dados baseados no campo VPI/VCI;

3. Interfaces capazes de multiplexar intervalos de tempo - TDM (Time-Division Multiplex) Interfaces que enviam dados com base em intervalos de tempo (time slots) de uma maneira cíclica. Como exemplo cita-se interfaces de um dispositivo cross-connect digital de um sistema SONET/SDH;

4. Interfaces capazes de comutar comprimentos de onda - LSC (Lambda Switch Capable) Interfaces que enviam dados com base nos comprimentos de onda nos quais o dado é 
recebido. Como exemplo, pode se citar uma interface de um OXC, operando no nível óptico;

5. Interfaces capazes de comutar fibras ópticas - FSC (Fiber-Switch Capable)

Interfaces, que enviam dados com base na posição de fibras ópticas em uma matriz de comutação. Como exemplo, cita-se uma interface de um OXC, operando no nível de uma ou múltiplas fibras ópticas;

Um circuito pode ser estabelecido somente entre, ou através de, interfaces de mesmo tipo. Dependendo da tecnologia utilizada em cada interface, diferentes denominações de circuitos podem ser usadas. Como exemplo temos, circuitos SONET/SDH e caminhos ópticos. No contexto GMPLS, todos estes circuitos são referenciados por um nome comum: LSP (label switched path). De fato, redes baseadas em GMPLS têm como objetivo encontrar e prover um LSP (caminho) ótimo baseado nos requerimentos de tráfego de um usuário. Tal LSP pode potencialmente começar em uma rede IP, ser transportado por uma rede SONET-SDH e então ser comutado através de um comprimento de onda específico em uma fibra óptica. Um diagrama de blocos do plano de controle GMPLS pode ser visto na Figura 4.2.

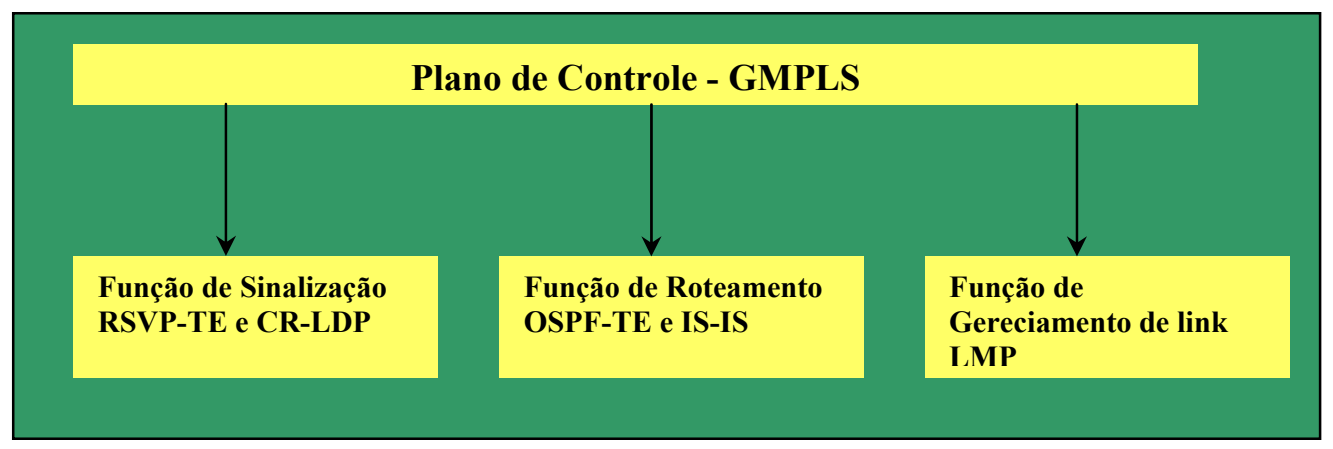
IS-IS - Interior System to Interior System
$\begin{array}{lr}\text { LMP - Link Management Protocol } & \text { OSPF - } \\ \text { RSVP - TE Resource reseration protocol - Traffic Engineering }\end{array}$
CR-LDP - Constraint-based Routing LDP

\section{FIGURA 4.2: Diagrama de blocos da arquitetura GMPLS}

Estes blocos são baseados em protocolos conhecidos de sinalização e roteamento com extensões para suportar GMPLS. Somente um novo protocolo é exigido, um protocolo de sinalização para gerenciamento de enlace (LMP-link management protocol). 
De fato, o uso de tecnologias como o DWDM possibilita a existência de um grande número de enlaces paralelos entre dois nós adjacentes. Para facilitar o gerenciamento desses enlaces, um conceito de empacotamento de enlace (link bundling, apresentado neste capítulo) é introduzido. Todavia, a configuração manual e o controle destes enlaces torna-se impraticável. Por isso, um novo protocolo, o LMP (link management protocol) foi especificado para resolver estas questões. Especificamente, o LMP provê mecanismos para manter a conectividade do canal de controle, verificar a conectividade física do feixe de enlaces (link verification), realizar um processo de correlação (para determinar inconsistências) de trocas de propriedades de enlaces (link property correlation) e gerenciar as falhas dos enlaces (fault link and fault notofication). $\mathrm{O}$ protocolo LMP é definido no contexto do GMPLS, mas é especificado independentemente da especificação de sinalização do GMPLS. Além disso, extensões para os protocolos de roteamento tradicionais são necessárias para codificar e carregar uniformemente informações de enlace, ou seja, propagar o estado e as restrições ao uso de certos recursos físicos e suas propriedades (identificadores de fibras ópticas, largura de banda e comprimentos de onda) entre dois nós GMPLS. Adicionalmente, a sinalização deve agora ser capaz de transportar os parâmetros requeridos pelo LSP tais como largura de banda, tipo de sinal, proteção desejada e posição em um dispositivo multiplex particular, para tecnologias específicas como, por exemplo, TDM (Time-Division Multiplex) e fibras ópticas. Assim, o GMPLS estende os dois protocolos de sinalização RSVP-TE e CR-LDP. Entretanto, a arquitetura GMPLS não especifica qual deles deve ser usado, sendo papel dos operadores de redes e seus fornecedores escolher qual deles melhor se adapta aos seus interesses.

A padronização da arquitetura GMPLS possui até o momento quatro RFCs (request for comment) designadas como: RFC 3471, 3472, 3473, 3474. Tal arquitetura é constituída basicamente de extensões dos protocolos de roteamento (IS-IS ou OSPF) e protocolos de sinalização (RSVP ou CR-LDP), além de um protocolo LMP. Neste trabalho optou-se por ressaltar os conceitos principais apontando as referências nas quais consta a forma de implementação destes conceitos, específicas para cada protocolo.

A seguir, nas Seções 4.2, 4.3 e 4.4 serão apresentados, respectivamente, o protocolo LMP, as extensões para os protocolos de sinalização (RSVP e CR-LDP) e as extensões para os protocolos de roteamento (OSPF e IS-IS). Na Seção 4.5 apresentar-se-á a maneira de utilizar estas extensões GMPLS para a configuração de LSP em redes, nas quais os rótulos são designados não apenas nos cabeçalhos de pacotes como também por intervalos de tempo, comprimentos de onda ou portas físicas. Na Seção 4.6 serão explicitados os modelos de interconexão coberto 
(overlay) e de pares (peer). Por fim na Seção 4.7 são discutidas algumas limitações da arquitetura GMPLS.

\subsection{PROTOCOLO DE GERENCIAMENTO DE ENLACE - LMP}

A conseqüência de generalizar o MPLS para abranger enlaces nos quais os rótulos não são anexados aos dados implica na existência de rótulos descritos como intervalos de tempo, comprimentos de onda e recursos físicos, tais como portas de um comutador (switch). Esta abordagem requer o mapeamento destes rótulos físicos entre nós adjacentes. No intuito de isolar falhas em enlaces TE (Traffic Enginneering), a arquitetura GMPLS implementa, como mencionado anteriormente, um protocolo de gerenciamento de enlace (LMP). Um enlace TE é uma representação utilizada por protocolos de roteamento (OSPF/IS-IS), na qual, além dos atributos normais de um enlace, são transportadas também restrições empregadas visando implementar a engenharia de tráfego, ou seja, impedir o roteamento baseado apenas no caminho mais curto. Tais restrições dizem respeito, por exemplo, ao tipo de proteção utilizado em um enlace e aos identificadores remotos e locais de cada enlace. Portanto, um enlace TE pode ser entendido como um conjunto de enlaces lógicos (um enlace composto por vários outros enlaces) com propriedades de engenharia de tráfego (TE), algumas das quais podem ser propagadas por LSRs e outras obtidas dos componentes físicos dos enlaces (como os comprimentos de onda utilizados).

O protocolo LMP é uma coleção de procedimentos entre nós adjacentes, os quais realizam serviços locais como os descritos abaixo. Os dois procedimentos iniciais são obrigatórios e os dois últimos opcionais. Detalhes sobre estes procedimentos são apresentados no Apêndice III.

1. Gerenciamento de canal de controle (control channel management)

Este procedimento é usado para estabelecer e manter canais de controles entre nós adjacentes;

2. Correlação da propriedade do link (link property correlation)

Este processo tem a função de detectar inconsistências relacionadas à propriedade do enlace entre dois nós adjacentes. Ou seja, agregar múltiplos enlaces de dados em um enlace TE e verificar a sincronização da propriedade destes entre o nó local e o 
adjacente. $\mathrm{O}$ canal de controle entre nós adjacentes não mais utiliza o mesmo meio físico que transporta os enlaces de dados;

3. Verificação da conectividade do enlace (link connectivity verification)

O processo de verificação da conectividade do enlace é um procedimento opcional, o qual pode ser empregado para verificar a existência de conectividade física dos enlaces entre dois nós adjacentes;

4. Gerenciamento de falhas (fault management)

O protocolo LMP provê uma função de localização de falhas em um ou mais canais de dados em um domínio óptico;

\subsection{EXTENSÕES GMPLS PARA OS PROTOCOLOS DE SINALIZAÇÃO}

A arquitetura GMPLS estende as funções dos protocolos de sinalização do MPLS e em alguns casos acrescenta funcionalidade. Estas mudanças e adições impactam propriedades dos LSPs, a maneira pela qual os rótulos são solicitados e comunicados, a natureza unidirecional dos LSPs e a forma como os erros são propagados.

O núcleo das especificações de sinalização da arquitetura GMPLS é disponível em três partes distintas :

1. Uma descrição funcional da sinalização [39]

2. Extensões ao protocolo de sinalização RSVP [40]

3. Extensões ao protocolo de sinalização CR-LDP [41]

Em adição, extensões independentes são disponíveis de acordo com a tecnologia:

I. Extensões para SONET e SDH [42]

II. Extensões segundo a norma G.709 [43 - Redes ópticas padronizados pelo ITU-T]

As extensões da arquitetura GMPLS para os protocolos de sinalização estão definidas na Figura 4.3 abaixo: 


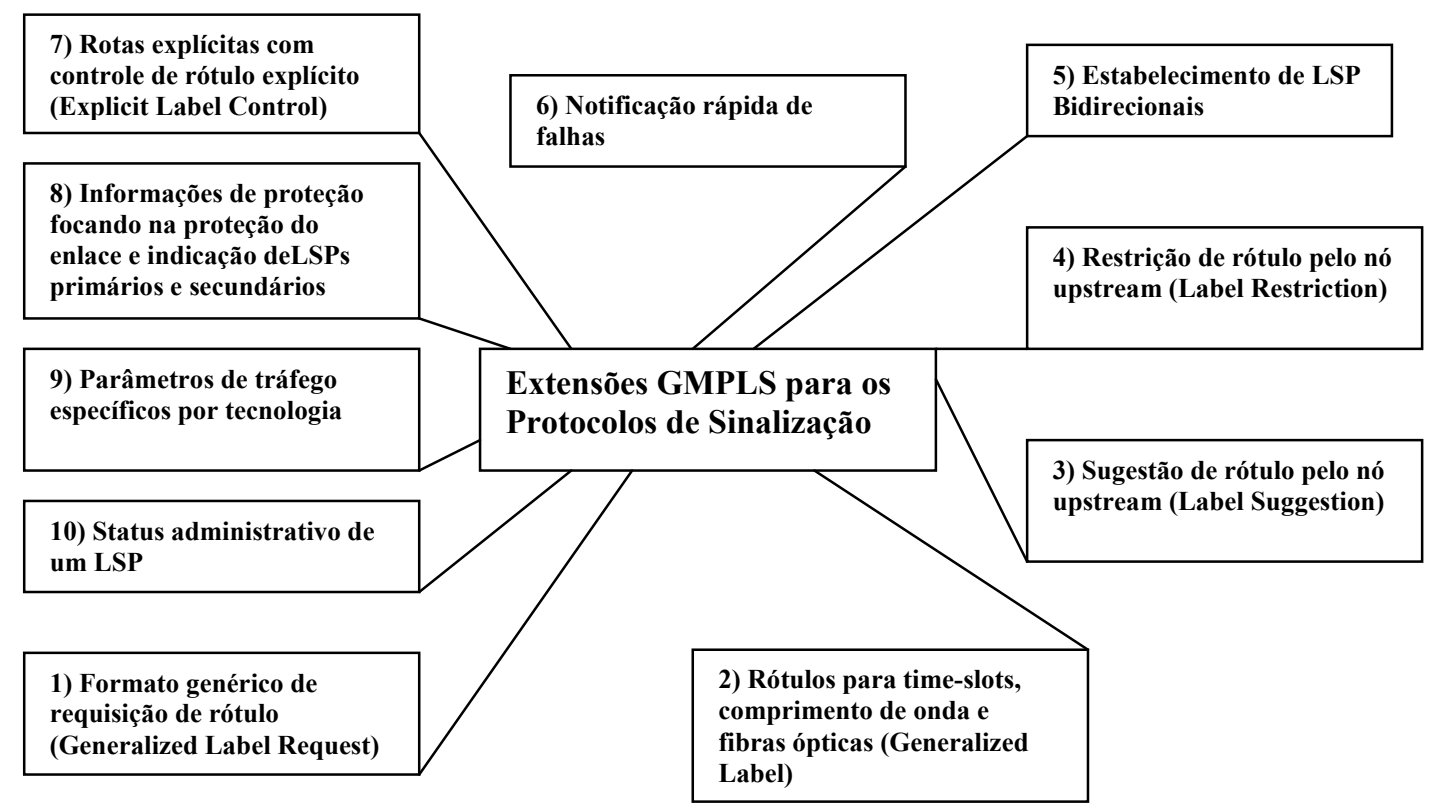

FIGURA 4.3: Extensões GMPLS para os protocolos de sinalização.

No tocante à sinalização, a arquitetura GMPLS define algumas extensões obrigatórias e outras opcionais, permitindo a constituição de diversas formas de implementar uma rede óptica. Somente as funções 1, 2 e 9 são obrigatórias. Tipicamente as funções 5 e 7 devem ser implementadas e as funções 3, 4, 6, 7, e 10 são opcionais. Uma rede SONET/SDH típica deveria implementar as funções: 1, 2 (com rótulos específicos para SONET/SDH), 5, 8, 9 e 10, as funções 6 e 8 são opcionais desde que proteção e restauração podem ser realizados usando os bytes de overhead da tecnologia SONET/SDH. Uma rede típica de comutação de comprimento de onda poderia implementar as funções: 1,2 (o formato genérico), 3, 4, 5, 6, 7, 8 e 10. Uma rede MPLS típica poderia não implementar nenhuma destas funções. Entretanto, as funções 1 e 8 podem ser usadas. Neste caso, a rede MPLS pode se beneficiar de um tipo de proteção de enlace não disponível usualmente. Nos tópicos relacionados abaixo cada um destes itens será discutido em detalhe.

\subsubsection{Formato genérico de requisição de rótulo (Generalized Label Request)}

Diferente das redes MPLS, em redes GMPLS, os dados contidos em um fluxo requisitado não são sempre associados a um valor de rótulo. De fato, o valor do rótulo é implícito, pois está sendo transportado por comprimentos de onda específicos, intervalos de tempo (time slots) ou 
fibras ópticas. Por outro lado, alguma representação do valor do rótulo é requisitada pelos protocolos de sinalização, para que mensagens de controle entre os equipamentos possam concordar com o valor e ser utilizado. O pedido de rótulo genérico (Generalized label request) introduz um objeto denominado Generalized label (RSVP) e um TLV (Type-Length-Value) Generalized label (CR-LDP) para carregar ambos, o rótulo e informações associadas. Como mencionado anteriormente, todas as mensagens trocadas pelo protocolo CR-LDP possuem uma estrutura comum que usa um esquema de codificação TLV. Já, para o protocolo RSVP, o esquema de codificação refere-se a um objeto. Somente um pedido de rótulo pode ser usado para cada mensagem. Desta maneira, um único LSP pode ser pedido por vez em uma mensagem de sinalização. A forma padrão e os parâmetros desta função são apresentados no Apêndice III.

Outros parâmetros específicos não são transportados em um pedido de rótulo genérico (Generalized label request), mas sim em parâmetros de tráfegos para tecnologias específicas. Atualmente dois conjuntos de parâmetros estão definidos, um para SONET/SDH [42] e um para G.709 (Redes Ópticas padronizadas pelo ITU-T) [43], que determina os parâmetros de tráfegos especificados por tecnologia.

\subsubsection{Rótulo Genérico (Generalized label)}

A função de rótulo genérico (Generalized label), consiste na resposta a um pedido de rótulo conforme descrito acima. Esta função estende o rótulo tradicional da arquitetura MPLS por assegurar a representação não somente de rótulos associados a pacotes, mas também de rótulos que identificam intervalos de tempo, comprimentos de onda, fibras ópticas ou portas de um comutador. Por exemplo, o rótulo genérico (Generalized label) pode identificar: a) uma única fibra em um empacotamento destas, b) um único comprimento de onda dentro de uma fibra, c) um intervalo de tempo (time slot) dentro de um comprimento de onda ou de uma fibra óptica. Um rótulo genérico (Generalized label) somente transporta um único nível de rótulo não hierárquico. Quando múltiplos níveis de rótulos (LSPs dentro de LSPs) são necessários cada LSP deve ser estabelecido separadamente. A representação de um rótulo genérico pode ser encontrada no Apêndice III. 


\subsubsection{Sugestão de Rótulo (Label suggestion)}

Como visto anteriormente, na arquitetura MPLS, o rótulo é designado no sentido do nó que esteja contra o fluxo de transmissão de dados (downstream) para o nó que esteja a favor (upstream). A função de sugestão de rótulo (label suggestion) é usada para informar a um nó downstream a preferência de rótulo de um nó upstream. Esta função é útil quando se estabelecem LSPs através de certos tipos de equipamentos ópticos, nos quais pode existir um atraso na configuração de sua matriz de comutação. Por exemplo, microespelhos (MEMS) necessitam ser fisicamente deslocados. Esta movimentação física e posterior amortecimento demandam um intervalo de tempo. Se o rótulo e a matriz de comutação são configurados no sentido reverso ao normal (downstream-upstream), há uma otimização do tempo utilizado para a configuração dos dispositivos MEMS, sendo este fato importante para propósitos de restauração de LSPs, que podem requer estabelecimento rápido como resultado de ocorrência de falhas na rede. O uso da função de sugestão de rótulo (label suggestion) é somente um aperfeiçoamento. Se o nó downstream transmite um rótulo diferente ao nó upstream, este reconfigura-se para utilizar o rótulo transmitido, mantendo assim o controle do rótulo pelo nó downstream. A transmissão de uma sugestão de rótulo (label suggestion) não implica em sua utilização para a transmissão de dados. Desta maneira, um nó upstream só poderá utilizá-lo após permissão do nó downstream.

\subsubsection{Restrição de Rótulo (Label restriction)}

Um nó upstream pode limitar a escolha de rótulos de um nó downstream em um conjunto de rótulos aceitáveis, enviando-lhe listas ou faixas de rótulos aceitáveis, os quais obedecem a algumas restrições. Existem três casos onde a restrição de rótulo no domínio óptico é útil:

1. onde um equipamento final é capaz de transmitir e receber somente em um pequeno conjunto de comprimentos de onda;

2. onde existe uma seqüência de interfaces, as quais não suportam conversão de comprimentos de onda e requerem o uso do mesmo comprimento de onda fim a fim em uma seqüência de nós, por todo o caminho; 
3. onde dois equipamentos finais de um enlace suportam diferentes conjuntos de comprimentos de onda;

O nó destinatário de um conjunto de rótulos deve restringir sua escolha a este conjunto. Um conjunto de rótulos pode estar presente através de múltiplos nós. Em cada um destes será gerado seu próprio conjunto de rótulos baseado no conjunto de rótulos enviado pelo nó anterior e seus próprios recursos de hardware. O formato típico de um conjunto de rótulos (label set) é apresentado no Apêndice III.

\subsubsection{Estabelecimento de LSP bidirecional}

Na arquitetura MPLS, os LSPs são unidirecionais. A fim de estabelecer um LSP bidirecional usando os protocolos de sinalização (RSVP e CR-LDP), dois LSPs unidirecionais em direções opostas devem ser estabelecidos independentemente. Tais LSPs apresentam algumas desvantagens: a latência para se estabelecer dois LSPs em direções contrárias é alta; o overhead de controle é o dobro de um LSP unidirecional, pois mensagens de controle devem ser geradas em ambos os segmentos dos LSPs, os recursos destinados aos LSPs são estabelecidos em segmentos separados, dificultando a seleção de rotas. Em LSPs bidirecionais não faz sentido falar em nó upstream ou downstream pois o fluxo de dados ocorre em ambas as direções. Utiliza-se assim o conceito de nó "iniciador" (initiator) e nó "terminador" (terminator). O GMPLS estende os protocolos de sinalização para suportar o estabelecimento de LSPs bidirecionais usando um conjunto simples de mensagens de sinalização. Para o estabelecimento de LSPs bidirecionais, dois rótulos são alocados. Isto é realizado pela presença de um novo objeto(RSVP)/TLV(CR-LDP) denominado Upstream label. A implementação é específica para cada protocolo de sinalização utilizado e maiores detalhes podem ser encontrados em [40] e [41].

\subsubsection{Notificação rápida de falhas}

A arquitetura GMPLS define algumas extensões para acelerar a notificação de falhas e outros eventos aos nós responsáveis por restaurar os LSPs com falhas e lidar com os erros.

a) Conjunto de rótulos aceitáveis (acceptable label set) para notificação de erros de rótulos. Existem situações em redes MPLS e GMPLS que resultam em mensagem de erros 
contendo uma indicação de valor de rótulo inaceitável (unacceptable label value). Quando estes casos ocorrem, pode ser útil ao nó gerador da mensagem de erro indicar nesta quais rótulos deveriam ser aceitos. A arquitetura GMPLS introduz a capacidade de transmitir tal informação via o conjunto de rótulos aceitáveis (acceptable label set). Este é carregada em mensagens de erros específicas em protocolos de sinalização [40], [41];

b) Expedição de notificação

Extensões do RSVP [40] permitem expedir notificação de falhas e outros eventos para determinados nós. Para o protocolo CR-LDP não existe tal mecanismo. Uma extensão, denominada mensagem de notificação (notify message), é utilizada para notificar outros nós da ocorrência de uma falha, notificação enviada não apenas aos nós vizinhos;

\subsubsection{Rotas explícitas com controle de rótulo explícito (explicit label control)}

Esta função estende o conceito de rotas explícitas de arquitetura MPLS ao permitir ao LSR de ingresso especificar o rótulo para um, alguns ou todos os enlaces de uma rota. Isto se torna útil, por exemplo, quando um LSR de ingresso deseja estipular que seja usado o mesmo comprimento de onda ao longo de todo o LSP, o que é desejável a fim de se evitar a conversão de comprimentos de onda. Rótulos explícitos (explicit labels) são especificados pelo LSR de ingresso como parte de uma rota explícita. Em cada LSR ao longo do caminho, qualquer rótulo explícito que é especificado em tal rota tem seu conteúdo analisado, sendo enviado para o próximo nó. O LSR que recebeu este rótulo explícito deve utilizar este rótulo. Caso não seja possível, a configuração do LSP deve ser interrompida.

\subsubsection{Proteção de enlace}

Informação de proteção de enlace é carregada em um novo e opcional objeto (RSVP)/TLV (CR-LDP), denominado Informação de proteção (protection information). Esta opção indica a proteção desejada para cada enlace de um LSP. Existem vários tipos de mecanismos de proteção, como por exemplo proteção 1+1, proteção 1:N e proteção 1:1, todos especificados no Apêndice III. 
O GMPLS propaga a capacidade de proteção de cada enlace em protocolos de roteamento. Desta maneira, algoritmos de determinação de caminhos podem levar esta informação em conta na configuração de um LSP. Informações de proteção indicam também se o LSP é primário ou secundário. Um LSP secundário é um backup para o primário. Os recursos de um LSP secundário normalmente não são usados até o primário falhar, apesar de poderem ser usados por outros LSPs, até a eventual falha ocorrer no LSP primário e este necessitar destes recursos, obtendo-os. As informações transportadas pelo objeto (RSVP)/TLV(CR-LDP) Informação de proteção (protection information) são descritas no Apêndice III.

\subsubsection{Proteção e Restauração}

A recuperação de uma falha na rede acontece em estágios, os quais incluem a detecção da falha, localização, notificação e a recuperação propriamente dita. A detecção da falha deve ser processada nas camadas mais próximas das falhas. Para as redes ópticas, esta é a camada óptica. Uma forma de apontar falhas nesta camada é detectar a perda do sinal óptico. A localização de falhas pode ser feita, como já visto, utilizando-se o protocolo LMP. A notificação de falhas pode ser realizada usando-se os protocolos de sinalização RSVP e CR-LDP. Uma vez que a falha tenha sido detectada, localizada e notificada, mecanismos de proteção e restauração são usados para consertar a avaria.

Estes mecanismos de proteção encarregam-se de reservar recursos entre os pontos protegidos antes da falha ocorrer. A conectividade depois de ocorrida a falha é alcançada pela execução de comutação entre os pontos protegidos para os recursos previamente alocados. Mecanismos de proteção visam proporcionar um tempo de reação mais rápido, podendo ser classificados pelo nível de redundância e distribuição de recursos, vistos anteriormente.

Mecanismos de restauração contam com protocolos de sinalização para coordenar ações de comutação durante a recuperação, podendo envolver reprovisionamento, isto é, a sinalização ocorre somente após uma falha ocorrer, ou pré-sinalização, isto é, a sinalização ocorre antes de uma falha ocorrer.

Proteção e restauração podem ser abordadas de duas maneiras, localmente ou nos pontos finais. A abordagem local é focada no local próximo da falha, tendo como objetivo reduzir o tempo de recuperação, como mostrado na Figura 4.4. Na abordagem fim a fim, os nós que originam e terminam o LSP controlam a recuperação. Detalhes da implementação de mecanismos de proteção e restauração podem ser encontrados em [40] e [41]. 


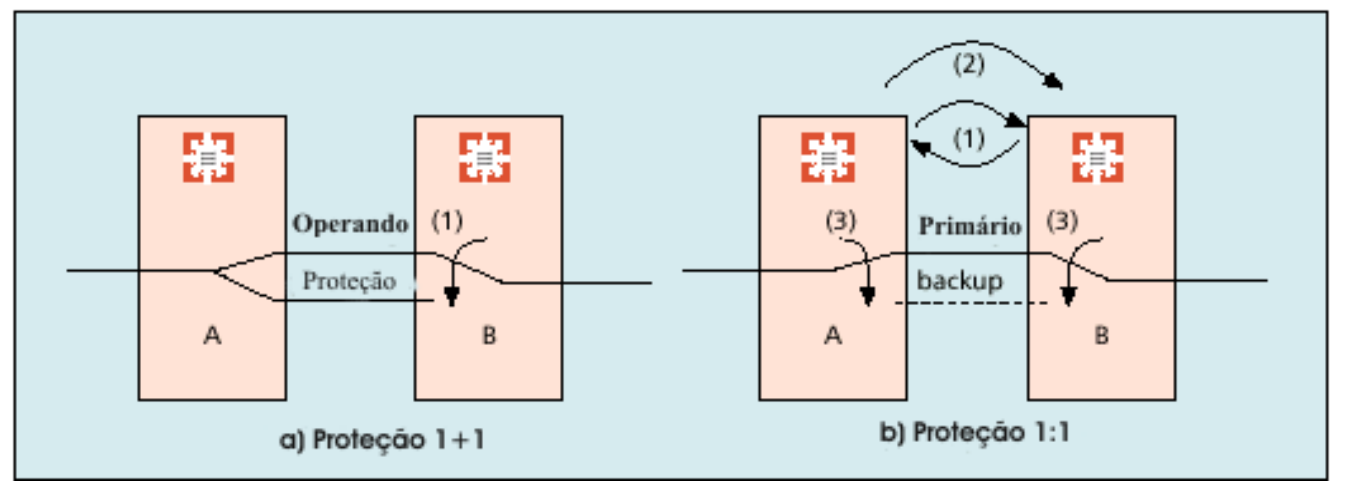

FIGURA 4.4:a) Em um mecanismo de proteção 1+1 uma conexão é transmitida simultaneamente sobre dois canais (um operando e outro de proteção) e um seletor é usado no nó destino para escolher o melhor sinal. Se uma falha ocorre (1) o nó destino comuta para o enlace de proteção. b) Em um mecanismo de proteção 1:1 um dedicado enlace de reserva é pré-alocado para o enlace primário. Se uma falha ocorrer (1) o protocolo LMP é usado para localizar a falha. Uma vez a falha tenha sido localizada (2) uma mensagem refresh (RSVP) pode ser usada para indicar o caminho a ser comutado e (3) ambos os nós deveriam comutar para o canal de reserva [44].

Nestas circunstâncias algum mecanismo é necessário para detectar falhas na comunicação através do canal de controle e um procedimento de recuperação deve garantir a integridade do canal de controle nas suas extremidades (nó inicial e final). No caso de uma falha no canal de controle, uma vez que a comunicação seja restaurada, protocolos de roteamento são capazes de recuperar o circuito, mas os protocolos de sinalização devem assegurar que qualquer mudança de estado (por exemplo nos LSPs) ocorrida durante a falha, seja sincronizada entre os nós. No caso de uma falha nodal, ambos os nós devem sincronizar as informações de estado deles com a do nó reiniciado. Para ocorrer a resincronização, o nó afetado necessitará preservar algumas informações, tais como o mapeamento dos rótulos de chegada para os de saída. Estas questões são discutidas especificamente para cada protocolo de sinalização [40], [41]. Em particular o protocolo de sinalização RSVP, após mapear o canal de controle para uma interface, pode descobrir a falha nesta através de mensagens de sinalização HELLO.

\subsubsection{Status Administrativo de um LSP}

A informação do status administrativo de LSP é transportada em um novo objeto (RSVP)/TLV (CR-LDP), utilizado em dois modos. No primeiro é indicado o estado de um LSP: se este está ativo ou inativo, se está em modo de teste ou se sua própria supressão está em 
progresso. A ação tomada pelos nós, baseada nestas informações, pode inibir a notificação de alarmes quando um LSP está inativo ou em teste. No segundo modo, a informação do status administrativo indica um pedido para configurar um estado administrativo para um LSP, por exemplo, um LSP de teste. Esta informação é sempre relatada pelo nó de ingresso, o qual originou o pedido. Os diferentes usos são formatados de maneiras especificas para os dois protocolos de sinalização. Para mais detalhes ver [40] e [41].

\subsection{EXTENSÕES GMPLS PARA OS PROTOCOLOS DE ROTEAMENTO (OSPF E IS-IS)}

A arquitetura GMPLS estende os protocolos de roteamento OSPF e IS-IS (Figura 4.5) com o intuito de proporcionar às redes com múltiplos enlaces de diferentes tecnologias (time slots, comprimentos de onda e pacotes) a autodescoberta de sua topologia e a propagação da disponibilidade de recursos presentes nesta. Detalhes da implementação destas extensões para os protocolos OSPF e IS-IS são apresentados respectivamente em [45] e [46].

1) Propagar o tipo de

proteção de um enlace
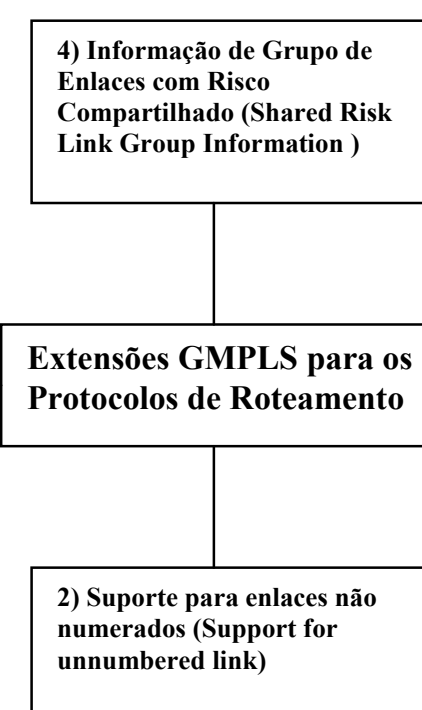

3) Descritor da

Capacidade de Comutação

de uma Interface

(Interface Switching

Capability Descriptor)

FIGURA 4.5: Extensões GMPLS para os protocolos de roteamento.

\subsubsection{Capacidade de Proteção de um enlace}

Torna-se desejável transportar a informação relacionada à capacidade de proteção de um enlace para que esta possa ser utilizada para a configuração de LSPs com características de 
proteção específicas. Os tipos de proteção são, assim, os mesmos utilizados pelos protocolos de sinalização, descritos no Apêndice III. Ou seja, proteção estendida (Enhanced), proteção dedicada (dedicated 1+1), proteção dedicada (dedicated 1:1), proteção distribuída (shared), sem proteção (unprotected) e extra tráfego (extra traffic). A função de informar a capacidade de proteção de um enlace é opcional.

\subsubsection{Suporte para enlaces não-numerados (Support for unnumbered links)}

Enlaces não-numerados são enlaces ponto a ponto, não identificados por endereços IP. Os protocolos de roteamento necessitam de um identificador para serem capazes de representá-los. Cada um dos LSRs presentes nas extremidades de um enlace não-numerado designa um identificador de 32 bits. A Figura 4.6 apresenta um enlace não-numerado entre os LSRs A e B. O LSR A escolhe um identificador para o enlace de interesse (42), B executa o mesmo procedimento (70). Da perspectiva do LSR A, o identificador por ele designado para o enlace denomina-se identificador de enlace local (LLI-link local identifier) e o identificador designado por B para o enlace chama-se identificador de enlace remoto (LRI-link remote identifier). O suporte a enlaces não-numerados inclui, assim, a troca de informações sobre os identificadores deste enlace. Esta troca pode ser realizada por meio dos protocolos de roteamento OSPF e IS-IS, pelo protocolo LMP ou pelos protocolos de sinalização RSVP [47] e CR-LDP [48].

A redução de esforços de gerenciamento de endereço IP através da aplicação deste fundamento torna-se um aspecto muito significativo, especialmente no contexto de redes ópticas, nas quais pode existir uma grande quantidade de enlaces entre nós.

\subsubsection{Descritor da Capacidade de Comutação de uma Interface (Interface Switching Capability Descriptor)}

No contexto GMPLS, um enlace é conectado a um nó por uma interface, a qual pode apresentar diferentes capacidades de comutação. Por exemplo, uma interface conectando um enlace a um nó pode ser capaz de comutar pacotes e não ser capaz de comutar comprimentos de onda. A cada interface é associado um descritor de sua capacidade de comutação (ISCDinterface switching capability descriptor) [49], sendo este descritor propagado pelos protocolos de roteamento e utilizado na computação de um caminho através da rede. Como visto anteriormente, os tipos de comutação possíveis para uma interface são: 


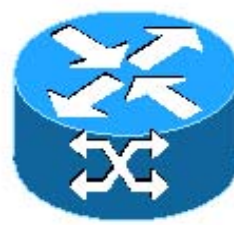

LSR A

Identificadores Local Remoto $42 \quad 70$
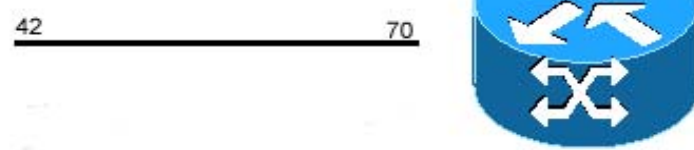

LSR B

Identificadores

Local Remoto

70

FIGURA 4.6: Suporte a enlaces não numerados com os identificadores (32 bits) locais e remotos.

1. Capacidade de comutar pacotes (packet-switch capable)

Um nó recebendo dados nesta interface pode comutá-los com base no rótulo transportado em seu cabeçalho;

2. Capacidade de comutar dados com base na camada 2 - L2SC (layer-2 switch capable) Um nó recebendo dados nesta interface pode comutá-los baseado no endereço da camada 2. Uma interface associada com um enlace terminando em um comutador (switch) ATM pode ser considerado um exemplo deste tipo de comutação;

3. Capacidade de multiplexar time-slots (time-division multiplex capable)

Um nó recebendo dados nesta interface pode multiplexar ou demultiplexar canais dentro de uma hierarquia SDH-SONET;

4. Capacidade de comutar comprimentos de onda (lambda-switch capable)

Um nó recebendo dados nesta interface pode reconhecer e comutar comprimentos de onda individuais;

5. Capacidade de comutar fibras ópticas

Um nó recebendo dados nesta interface pode comutar o conteúdo inteiro de uma fibra óptica para outra, sem distinguir comprimentos de onda, time-slots ou pacotes. A uma 
interface deste tipo não é permitido extrair um comprimento de onda individual e comutá-lo;

Uma interface pode ter mais do que um descritor de sua capacidade de comutação (ISCD), sendo este processo usado para lidar com interfaces que suportam múltiplas capacidades de comutação. Por exemplo, considere um enlace de fibra óptica transportando um conjunto de comprimentos de onda, os quais têm como ponto final um LSR cuja interface pode comutar um destes comprimentos de onda para um canal óptico de saída ou pode extrair (demultiplexar) os dados deste comprimento de onda usando intervalos de tempo (time-slots) e então comutá-los para uma saída utilizando a multiplexação por divisão de tempo (TDM).

\subsubsection{Informação de Grupo de Enlaces com Risco Compartilhado (Shared Risk Link Group Information )}

A função de informar o grupo de enlaces com risco compartilhado é opcional. Um conjunto de enlaces constitui um grupo de enlaces com risco compartilhado (SRLG - shared risk link group) se compartilham um recurso cuja falha pode afetar todos os enlaces do conjunto. Por exemplo, duas fibras ópticas instaladas em um mesmo duto podem estar no mesmo SRLG. Um SRLG é identificado por um número de 32 bits que é único dentro do domínio de um protocolo de roteamento. Esta função pode ser empregada para a configuração de caminhos de backup. Tais caminhos não devem compartilhar os recursos pertencentes aos enlaces, para os quais funciona como proteção. Em outras palavras, os enlaces utilizados pelos caminhos de backup não devem possuir o mesmo SRLG dos enlaces que funcionam como proteção.

\subsection{EXTENSÕES GMPLS DA ARQUITETURA MPLS}

Nesta seção serão abordadas as formas pelas quais as extensões GMPLS à arquitetura MPLS podem ser utilizadas para prover um plano de controle capaz de comutar time slots, comprimentos de onda e portas de um comutador. Pode-se fazer algumas considerações, tais como [50]: 
1. Diversidade de Comutação

Enquanto, na arquitetura MPLS, o caminho percorrido por um LSP pode incluir diferentes tipos de enlaces (enlaces apenas entre roteadores, enlaces entre roteadores e LSRs-ATM), cada um realizando uma operação sobre o rótulo (cabeçalho de pacote ou campos em quadros como o VPI/VCI em ATM), a arquitetura GMPLS estende este conceito para incluir enlaces onde o rótulo é codificado como um intervalo de tempo, um comprimento de onda, ou uma posição em um feixe de fibras ópticas;

2. Diversidade de Envio

Os dados de usuários transportados em um domínio óptico são comutados de forma transparente para aumentar a eficiência da rede. Em outras palavras, nem todos os dispositivos presentes nas redes ópticas são capazes de ter acesso ao conteúdo dos dados transmitidos;

3. Alocação de Banda e Escalabilidade

Diferentemente das redes de comutação de pacotes, em redes utilizando interfaces capazes de comutar intervalos de tempo (time-slots), comprimentos de onda e fibras ópticas, a alocação de banda para um dado LSP pode ser realizada somente de um modo discreto. Por exemplo, uma rede de comutação de pacotes pode ter taxas de 10, 100 ou 1000 Mbps. Entretanto, uma rede óptica utilizará enlaces com largura de banda fixa, tais como 2,5 Gbps por comprimento de onda. Quando um LSP de 10 Mbps proveniente de uma rede de comutação de pacotes deve ser transportado por conexões ópticas, pode não fazer sentido alocar um canal de 2,5 Gbps para este LSP. Por outro lado, escalabilidade é uma questão importante quando se leva em conta a maneira de como as redes respondem às mudanças em sua topologia de uma forma rápida. Os recursos a serem gerenciados em redes ópticas podem ser mais complexos do que aqueles utilizados em redes baseadas em comutação de pacotes. É esperado que centenas de milhares de comprimentos de onda sejam transportados através de centenas de fibras; 
4. Configuração dos Comutadores

Configurar os comutadores ópticos ao longo de uma rota é um processo que demanda tempo. O intervalo de tempo utilizado para configurar um LSP nestas condições pode frustrar o desejo de utilizar-se deste para, por exemplo, reestabelecer a conexão após a ocorrência de uma falha;

\subsubsection{Maneiras de se implementar as extensões GMPLS}

A implementação de cada uma das considerações descritas acima é discutida respectivamente pelos itens desta seção.

\subsubsection{Diversidade de Comutação}

Para ser capaz de suportar dispositivos comutando em diferentes domínios, a arquitetura GMPLS provê novas extensões, como visto anteriormente, para a requisição e estabelecimento do rótulo genérico (generalized label request) e (generalized label).

O estabelecimento de um LSP em redes GMPLS é um processo similar ao utilizado em redes MPLS. A Figura 4.7 mostra a configuração de um LSP através de múltiplos enlaces de diferentes tecnologias. A largura de banda requerida pelo LSP1 é de $100 \mathrm{Mb} / \mathrm{s}$, devendo o caminho ter suficiente largura de banda em toda a sua extensão para suportar tal exigência. $O$ LSR $\mathrm{R}_{0}$ mapeia os pacotes para o LSP1. Este será agrupado pelo LSR $\mathrm{R}_{1}$ no $\mathrm{LSP}_{2}$ com taxa de $155 \mathrm{Mb} / \mathrm{s}$ (STM-1). O comutador (switch) $\mathrm{S}_{2}$ multiplexa o feixe de $155 \mathrm{Mb} / \mathrm{s}$ e o disponibiliza ao $\mathrm{LSP}_{3}$ o qual apresenta a taxa de $622 \mathrm{Mb} / \mathrm{s}$ (STM-4 entre $\mathrm{S}_{2}$ e $\mathrm{O}_{3}$ ), escolhe um comprimento de onda e o envia ao comutador óptico $\mathrm{O}_{3}$. Este comutador óptico $\left(\mathrm{O}_{3}\right)$ recebe o comprimento de onda selecionado em uma de suas portas e o comuta através do $\mathrm{LSP}_{4}$, por um canal DWDM até $\mathrm{P}_{4}$. Este é comutado através de $\mathrm{P}_{5}$ e $\mathrm{P}_{6}$ até $\mathrm{O}_{7}$. Deste modo, $\mathrm{O}_{7}$ seleciona o comprimento de onda correto e o transmite à porta adjacente do comutador $\mathrm{S}_{8}$. 


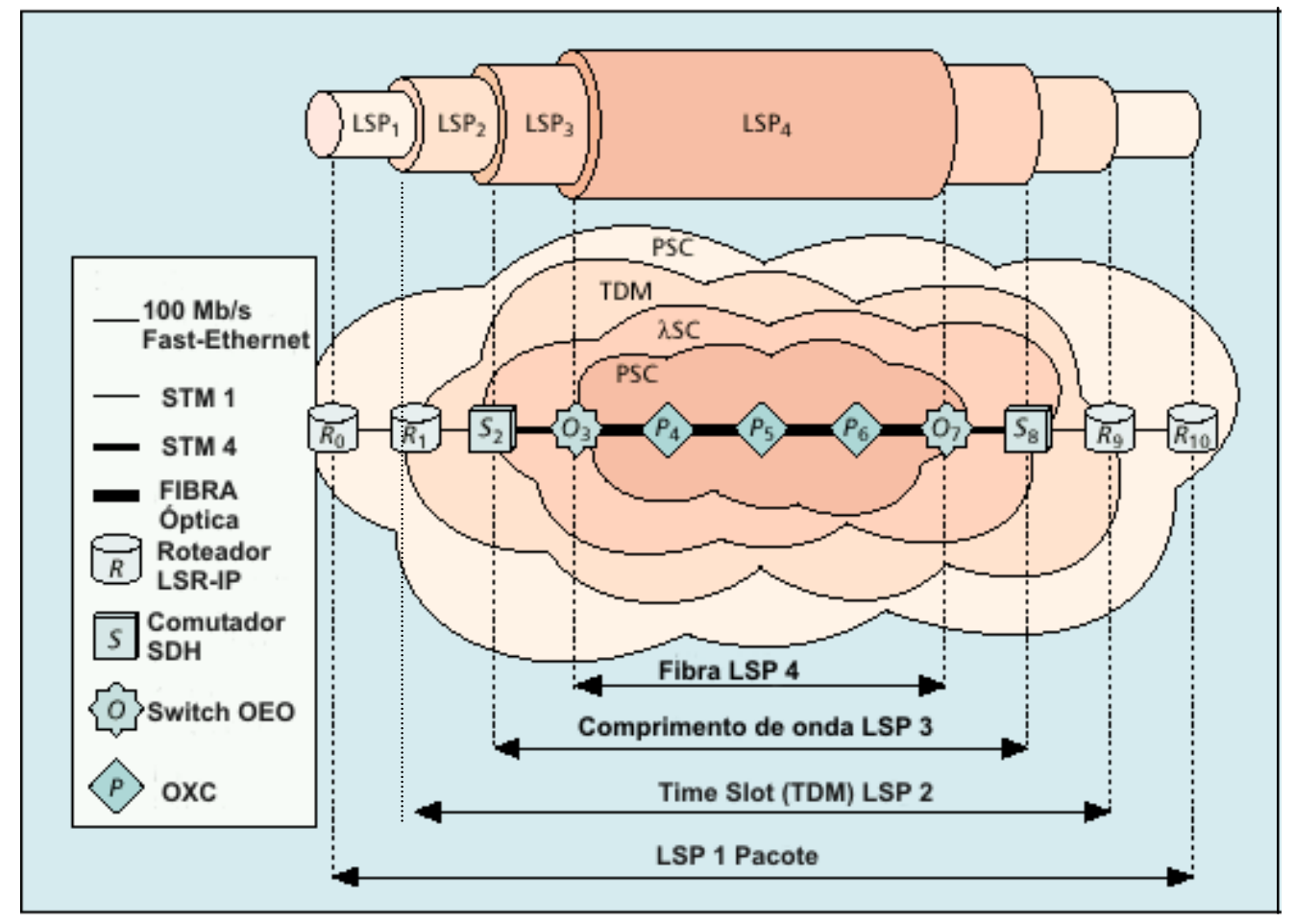

FIGURA 4.7: $O$ LSPı é configurado de $R_{0}$ até $R_{10}$ com a largura de banda de $100 \mathrm{Mb} / \mathrm{s}$. O LSPı é agrupado nos LSPs 2,3 e 4, respectivamente. O roteador $R_{0}$ mapeia os pacotes para o LSPı. Os roteadores $\mathbf{R}_{1}$ e $\mathbf{R}_{9}$ comutam pacotes. $\mathbf{S}_{2}$ e $\mathbf{S}_{8}$ são comutadores (switches) $\mathbf{S D H}$. Os dispositivos $\mathrm{O}_{3}$ e $\mathrm{O}_{7}$ são comutadores ópticos. Sua função é comutar o enlace STM-4 recebido em forma de um comprimento de onda (lambda) para um sistema DWDM, o qual será comutado pelos OXCs $\mathbf{P}_{4}$ a $P_{6}$. O enlace entre $R_{0}$ e $R_{1}$ é Fast-Ethernet, entre $R_{1}$ e $S_{2}$ um STM-1, entre $S_{2}$ e $O_{3}$ um STM-4 especificado em um comprimento de onda, entre $O_{3}$ e $P_{4}$ um sistema DWDM com 16 comprimentos de onda, cada um capaz de transportar um STM-4. Tais comprimentos de onda são comutados pelos OXCs. $O$ enlace entre $O_{7}$ e $S_{8}$ é um STM-4 recebido em forma de um comprimento de onda, entre $S_{8}$ e $\mathbf{R}_{9}$ um $\mathbf{S T M - 1}$ e entre $\mathbf{R}_{9}$ e $\mathbf{R}_{10}$ Fast-Ethernet.

O comutador $\mathrm{SDH} \mathrm{S}_{8}$ recebe o referido comprimento de onda, demultiplexa o sinal, selecionando o STM-1 desejado dentre aqueles que constituem o nível hierárquico STM-4 e o transmite para o LSR $\mathrm{R}_{9}$. Finalmente, o LSR $\mathrm{R}_{9}$ recebe os pacotes do STM-1 e os envia para o $\operatorname{LSR~R}_{10}$.

Deve-se notar que a banda disponível na hierarquia LSP é propagada pelos protocolos de roteamento. Ou seja: 
1. O roteador $R_{1}$ anuncia a capacidade do enlace de $R_{1}$ a $R_{10}$ com largura de banda igual a diferença entre o STM1 (155 Mb/s) e os $100 \mathrm{Mb} / \mathrm{s}$ alocados para o LSP1;

2. O comutador (switch) $\mathrm{S}_{2}$ anuncia o equivalente a 3 STM-1 como largura de banda disponível;

3. O comutador óptico $\mathrm{O}_{3}$ anuncia 15 comprimentos de onda, cada um com capacidade de comutar um STM-4;

O processo de sinalização para o LSP1 é descrito na Figura 4.8 utilizando-se, como exemplo, o protocolo de sinalização RSVP e suas extensões definidas na arquitetura GMPLS.

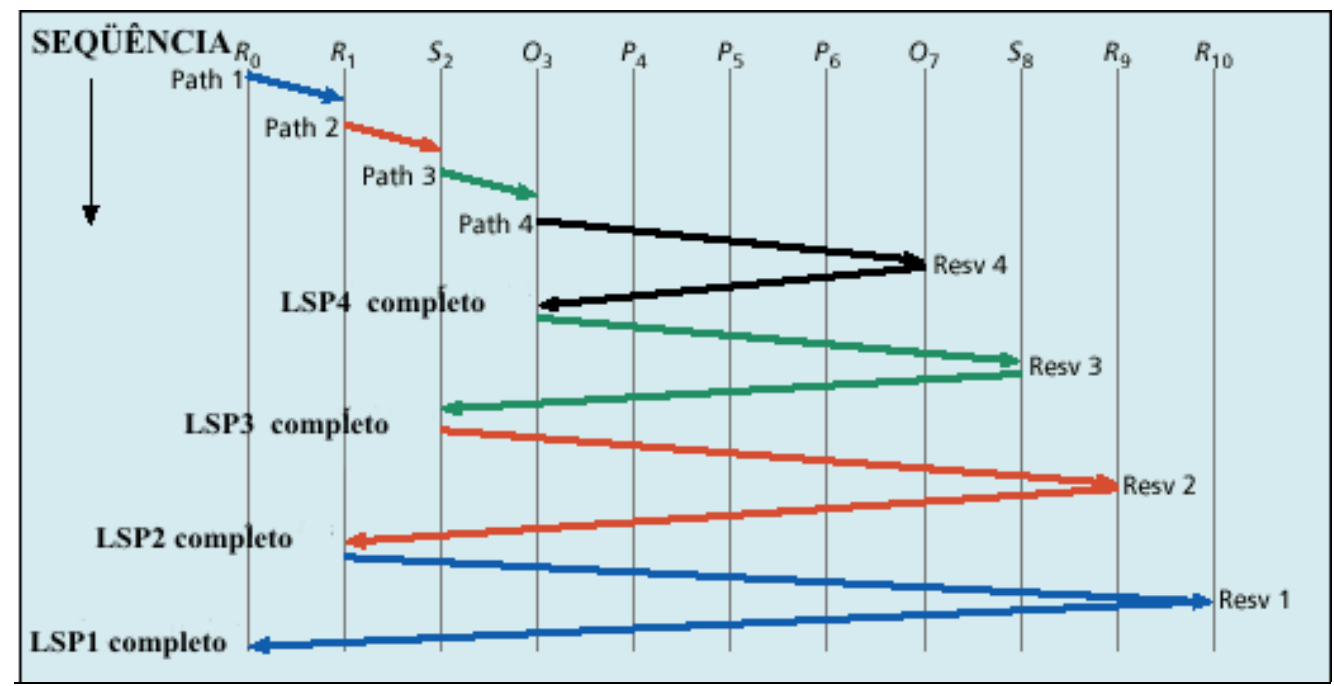

FIGURA 4.8: Uma mensagem PATH Request (path 1) é gerada no roteador $R_{0}$ e enviada a $\mathbf{R}_{1}$. No roteador $\mathbf{R}_{1}$ este pedido dispara uma solicitação para um novo LSP $\left(\operatorname{LSP}_{2}\right)$ de $\mathbf{R}_{1}$ a $\mathbf{R}_{9}$. Esta dinâmica de criação é repetida até a mensagem path 4 ser gerada na switch $O_{3}$. Seguindo o sucesso na configuração do $\mathrm{LSP}_{4}$ traduzido pelo recebimento da mensagem Resv, o $\operatorname{LSP}_{3}$ é tunelado através do $\mathrm{LSP}_{4}$. Este processo de formação de LSP, na qual um pedido de LSP de baixa ordem sendo canalizado através de um LSP de mais ordem já estabelecido, continua até o LSP inicial (LSPI) ser configurado.

Um LSP, de uma maneira geral, é estabelecido pelo envio de mensagens Path (RSVP)/Label Request (CR-LDP) para o nó de destino (nó downstream). Estas mensagens contêm um pedido de rótulo genérico (generalized label request) com o tipo de LSP (i.e. a camada envolvida) e seu tipo de carga, bem como parâmetros específicos tais como informação de proteção (protection 
information). Adicionalmente, uma sugestão de rótulo (label suggestion), um conjunto de rótulos (label set), um Upstream label (se o LSP é bidirecional) e a informação do status administrativo do LSP podem também ser incluídos nesta mensagem. Uma rota explicita é normalmente adicionada à mensagem pelo primeiro LSR. O nó downstream retornará uma mensagem Resv (RSVP)/label mapping (CR-LDP) incluindo um rótulo genérico (generalized label) com o intuito de configurar o LSP.

\subsubsection{Diversidade de Envio}

Dispositivos utilizados em redes MPLS são capazes de analisar as informações presentes no cabeçalho de um pacote. Eles examinam o rótulo do pacote para determinar a interface e o rótulo de saída deste. A arquitetura GMPLS estende este paradigma para aqueles dispositivos presentes em um domínio óptico, nos quais os dados são comutados transparentemente para aumentar a eficiência da rede. Neste caso, a arquitetura GMPLS permite ao plano de dados ser separado fisicamente do plano de controle, possibilitando a criação de canais de controle, nos quais são transmitidas as mensagens de sinalização e roteamento. Por exemplo, um canal de controle pode usar um comprimento de onda ou fibra óptica específicos, um enlace Ethernet, etc. A conseqüência de se implementar um canal de controle fisicamente separado do enlace de transporte de dados vem ao encontro da abordagem proposta pela arquitetura GMPLS, na qual o plano de controle é independente do plano de envio de dados.

\subsubsection{Alocação de Banda e Escalabilidade}

Em redes GMPLS, os fluxos de dados com taxas de bits baixas podem ser aninhados em fluxos com taxas de bits mais altas. Este fato traz a noção de uma hierarquia de LSPs. Esta hierarquia de LSPs é a noção segundo a qual LSPs podem ser agregados dentro de outros LSPs.

Uma hierarquia natural existe para ditar a ordem na qual LSP podem ser aninhados. Esta hierarquia (Figura 4.9) é baseada na capacidade de multiplexação dos tipos de LSPs. Tais LSPs sempre começam e terminam em equipamentos similares (um LSP baseado em comprimentos de onda origina-se e termina em um dispositivo comutador de comprimentos de onda). Em ordem decrescente nesta hierarquia estão os dispositivos que apresentam interfaces capazes de comutar fibras (FSC), os dispositivos com interfaces capazes de comutar comprimentos de onda, os dispositivos com interfaces capazes de comutar intervalos de tempo (time slots) e os 
dispositivos com interfaces capazes de comutar pacotes (PSC). Ou seja, fluxos compostos por pacotes com taxas de bits de, por exemplo, 10 Mbps podem ser multiplexados no tempo (TDM) com taxas de bits de $155 \mathrm{Mbps}$, estes podem ser comutados em comprimentos de onda com taxas de 2,5 Gbps e estes alocados em fibras ópticas. Este procedimento otimiza a alocação de banda em redes ópticas.

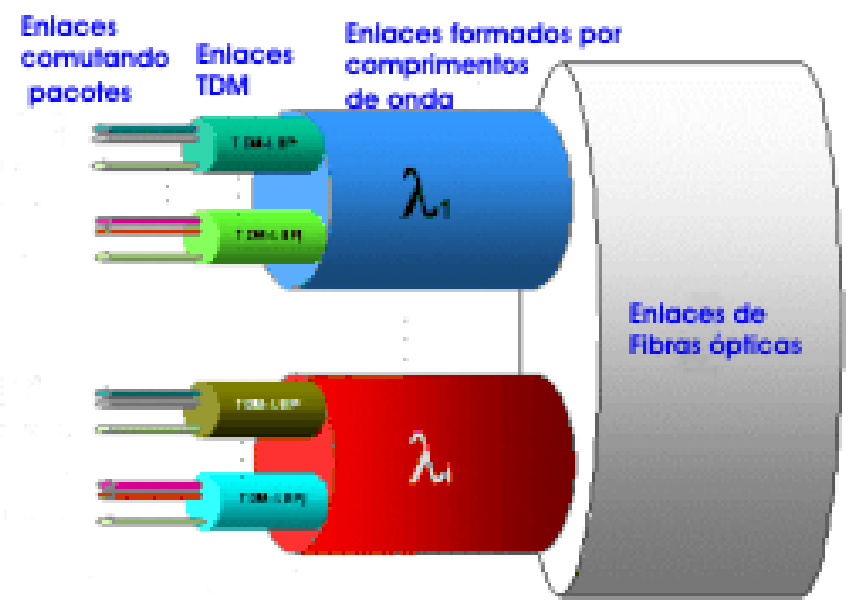

FIGURA 4.9: Hierarquia de LSPs

Com o intuito de melhorar a escalabilidade, a arquitetura GMPLS desenvolve o conceito de LSP formados por uma adjacência de envio (Forwarding Adjacency-LSP (FA-LSP)). Um FALSP é um enlace entre dois nós, os quais não são vizinhos mas pertencem à mesma instância, ou seja, às bordas de uma topologia pertencendo a uma mesma tecnologia (Figura 4.10), sendo propagados pelos protocolos de roteamento (OSPF e IS-IS) como um enlace igual a qualquer outro enlace físico. Devido a este método, cada nó, quando executa computação de rota, é capaz de usar não somente as informações dos enlaces convencionais, mas também de FA-LSPs. Em outras palavras, ao se agregar LSPs de baixa ordem em LSPs de mais alta ordem, os nós intermediários vêem apenas o LSP externo, não mantendo em suas bases de dados os estados de envio para LSP internos, aumentando consideravelmente a escalabilidade [51]. De fato, os círculos (por exemplo nuvens TDM ou FSC presentes na Figura 4.10) representam as interfaces em dispositivos de mesma natureza. Por exemplo, o círculo FSC consiste de OXC capazes de comutar fibras ópticas inteiras. O círculo LSC (lambda-switch capable) consiste de OXCs ou 
OADM capazes de comutar comprimentos de onda. O círculo TDM consiste de cross-conects SONET-SDH. Finalmente, o círculo PSC (packet-switch capable) consiste de roteadores IP. OS LSPs de baixa ordem são aninhados em LSPs de mais alta ordem e anunciados pelos IGPs (internet gateway protocol). Isto assegura que os LSPs de baixa ordem sejam agrupados e hierarquicamente canalizados através de LSPs de ordem mais alta. Múltiplos PSC-LSPs são canalizados dentro de um LSP-TDM, os quais são agrupados e tunelados dentro de um LSP-LSC e assim por diante. Na borda final de cada nuvem, tais LSPs são separados apropriadamente.

Como dito anteriormente, os protocolos de roteamentos (IS-IS/OSPF) distribuem as informações sobre os FA-LSPs da mesma maneira que distribuem as informações sobre qualquer outro enlace. Como resultado, um LSR tem em seu banco de dados de estado de enlace as informações sobre os enlaces, bem como sobre os FA-LSP. Sendo assim, uma decisão de roteamento é baseada em ambos. Em outras palavras, um FA-LSP é, por definição, um enlace TE (Traffic Engineering) entre dois nós GMPLS em cujo caminho transitam um ou mais nós na mesma instância de um plano de controle.

A arquitetura GMPLS também introduz, visando também melhorar a escalabilidade, o conceito de empacotamento de enlace (link bundling). Um exemplo típico ocorre em redes ópticas, nas quais nós adjacentes podem ser conectados por centenas de comprimentos de onda. Nestas redes, considerando a aplicação de protocolos de roteamento (OSPF ou IS-IS), cada comprimento de onda deve ser propagado, em princípio, separadamente. Ou seja, cada comprimento de onda origina uma entrada na tabela de roteamento destes protocolos. Também quando um par de LSRs é conectado por múltiplos enlaces, é possível propagar alguns ou todos destes como enlaces simples, em um processo chamado empacotamento de enlaces (link bundling) .

O resultado é um enlace lógico chamado enlace empacotado (bundled link) formado por enlaces físicos denominados componentes de enlaces. Os componentes físicos são constituídos por três identificadores (bundled link identifier, component link identifier, label), os quais, de maneira inequívoca, identificam os recursos utilizados por um LSP. O propósito deste empacotamento de enlace é melhorar a escalabilidade de roteamento, reduzindo a quantidade de informações manipuladas pelos protocolos. Com este intuito, todos os enlaces a serem empacotados devem começar e terminar em um mesmo par de nós, constituindo assim um enlace ponto a ponto, com as mesmas métricas de engenharia de tráfego (por exemplo, tipo de 
proteção) e a mesma capacidade de comutação (pacotes, time-slots , comprimentos de onda). Os detalhes de como os atributos dos enlaces são empacotados podem ser encontrados em [52].

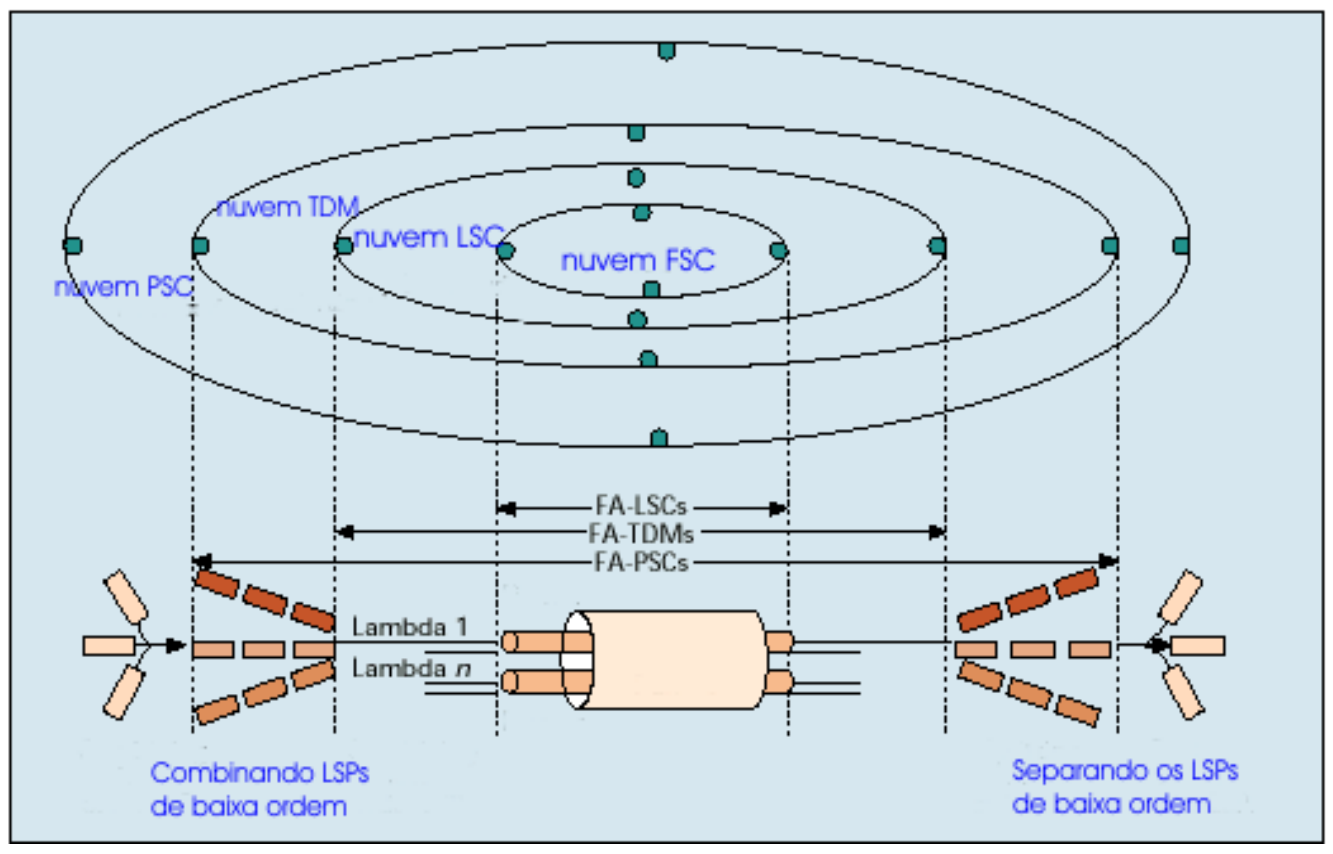

FIGURA 4.10: Hierarquia de LSPs, formando os respectivas FA-LSP (forwarding adjacency-LSP), os quais são processadas nas bordas de cada nuvem pertencente a uma mesma tecnologia.

\subsubsection{Configuração dos Comutadores}

Ao se estabelecer um LSP em redes utilizando a arquitetura GMPLS, pode ser necessário o estabelecimento de outros LSP intermediários. Tais LSP podem ser estabelecidos através de dispositivos ópticos (por exemplo, OXCs). Estes dispositivos apresentam características internas próprias, com as quais o GMPLS deve trabalhar, com o intuito de estabelecer um LSP fim a fim. Uma destas características é o tempo empregado pelos dispositivos para configurar sua matriz de comutação (por exemplo, através da movimentação de microespelhos-MEMS). Visando diminuir este tempo de latência, a arquitetura GMPLS utiliza, como visto anteriormente, a função de sugestão de rótulo (label suggestion), usada para informar um nó downstream sobre a preferência de rótulo de um nó upstream. Isto permite ao nó upstream configurar seu hardware com o rótulo sugerido, antes deste ser comunicado pelo nó downstream. Entretanto, se o nó downstream rejeitar o rótulo sugerido e oferecer o seu próprio, o nó upstream deve reconfigurarse de maneira a utilizar o novo rótulo. 


\subsection{MODELOS DE INTERCONEXÃO}

A arquitetura GMPLS disponibiliza dois modelos de interconexão, ainda em desenvolvimento, denominados: modelo coberto (overlay) e modelo de pares (peer) (Figura 4.11 a) e b)). Estes modelos apresentam diferentes procedimentos para a solicitação de serviços à rede GMPLS.

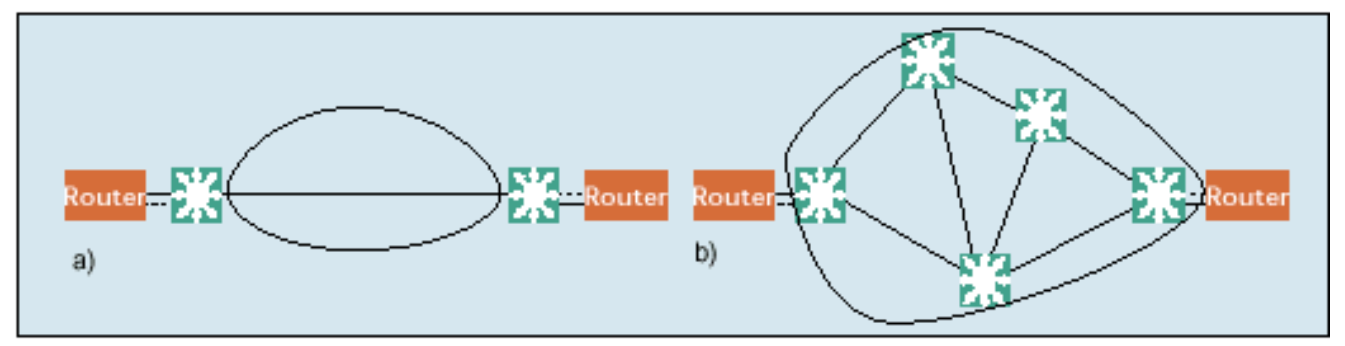

FIGURA 4.11: Modelos de interconexão; a) modelo coberto (overlay), b) modelo de pares (peer).

\subsubsection{Modelo de Pares (Peer)}

O modelo de pares (peer) torna o núcleo de uma rede óptica acessível, permitindo aos dispositivos de borda participar das decisões de roteamento, eliminando a barreira artificial entre o domínio de transporte e roteamento. Tendo o dispositivo de borda (cliente) acesso à topologia da rede, os mesmos protocolos de sinalização empregados na arquitetura GMPLS são utilizados para configurar um LSP através da rede. Em outras palavras, por obter acesso à completa topologia da rede, não há necessidade de novos protocolos para a configuração de um LSP (Figura 4.12).

Enquanto este modelo provê uma única camada de gerenciamento para o provedor de serviço, existem algumas considerações a serem feitas:

1. O provedor de serviço pode desejar manter a informação sobre a topologia do núcleo da rede apenas de seu conhecimento;

2. Provedores de serviço podem desejar manter os LSPs configurados tão estáveis quanto possível, gerenciados através de uma base de dados global da rede, com o intuito de prover proteção e restauração. Assim, poderiam não desejar automatizar a solicitação de um LSP por um dispositivo de borda (cliente); 


\section{GMPLS}

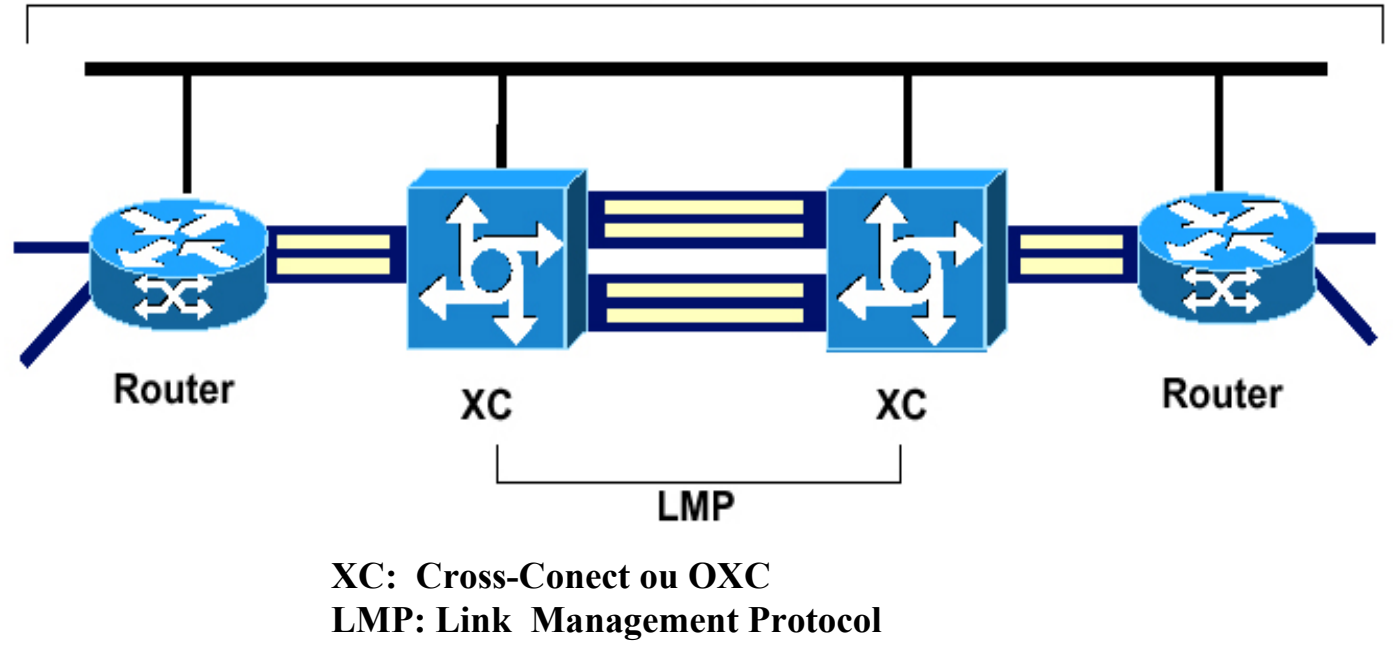

FIGURA 4.12: Modelo de pares (peer) no qual se constata a utilização de protocolos de sinalização disponíveis na arquitetura GMPLS.

\subsubsection{Modelo Coberto (Overlay)}

O modelo coberto (overlay) não permite aos dispositivos de borda acessar os detalhes internos de uma rede. Ou seja, o núcleo e a borda de uma rede são gerenciados separadamente, apresentando diferentes protocolos de sinalização. Em virtude deste fato, o modelo coberto (overlay) estimula a parceria entre duas grandes corporações voltadas à padronização, o OIF (Optical Internetworking Fórum) e o IETF.

Como resultado desta parceria, desenvolveu-se uma interface denominada UNI (user to network interface - OIF), a qual permite aos dispositivos clientes da rede a solicitação dinâmica de conexões através desta (Figura 4.13).

Um túnel entre os nós finais (UNI-N) é configurado usando protocolos de sinalização disponíveis na arquitetura GMPLS ou algum mecanismo proprietário de sinalização. O caminho de dados entre os nós clientes é configurado através deste túnel. A fim de configurar uma conexão, um cliente necessita descobrir quais serviços estão disponíveis, e assim sinalizar seus requerimentos através do UNI. Os tipos de parâmetros de serviços sinalizados através do UNI são [53]:

1. Pedido de banda para conexão;

2. Classe de serviço (ex. nível de proteção);

3. Características específicas de plano de dados (por exemplo, para redes SONET/SDH, informações de concatenação); 


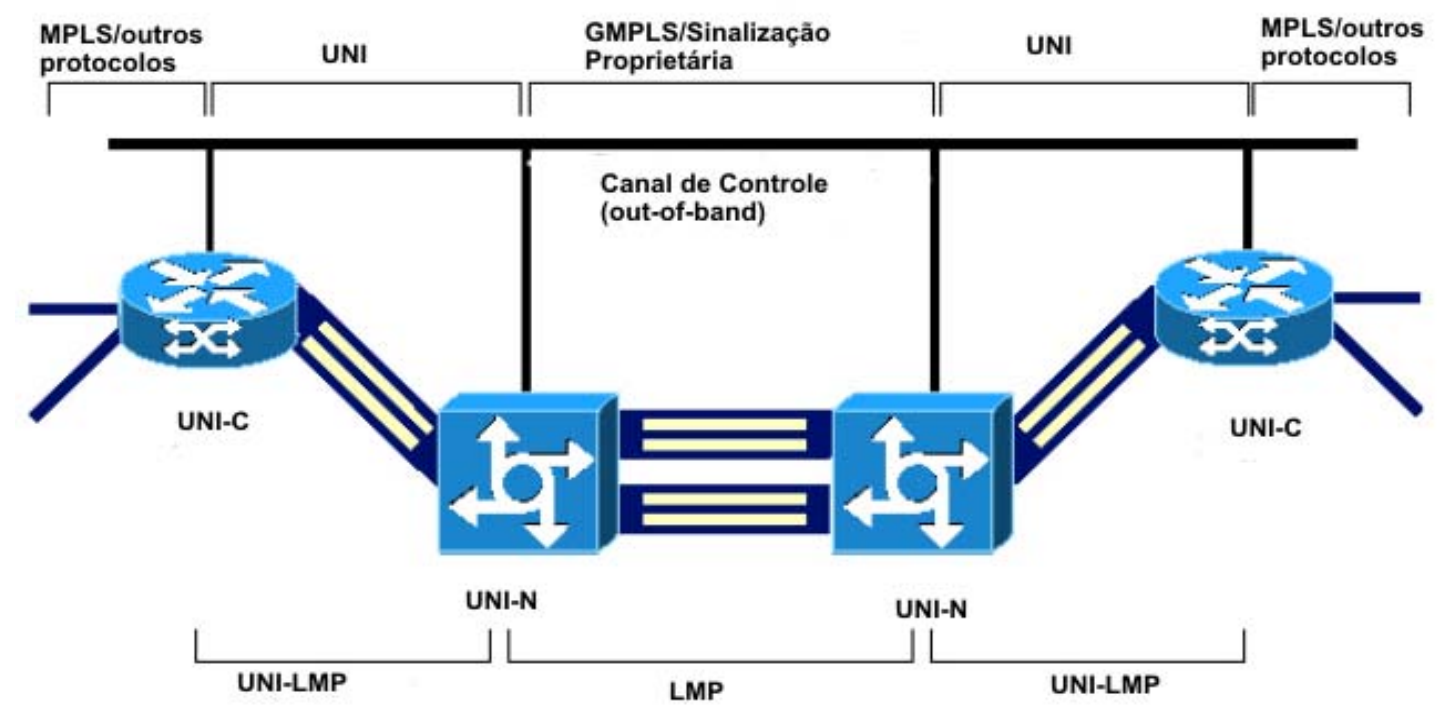

FIGURA 4.13: Modelo coberto (overlay), no qual se constata a utilização de interfaces UNI ( CCliente e N-rede padronizados pela OIF) e protocolos de sinalização presentes na arquitetura GMPLS (padronizado pelo IETF).

Uma característica fundamental da UNI é não permitir aos clientes o acesso às informações da topologia ou endereçamento da rede. Uma solicitação de cliente deve identificar os pontos extremos de uma conexão, a fonte (ele próprio) e o destino, usando algum esquema de endereçamento. De acordo com esta estratégia, é designado um endereço TNA (transport network assigned address) a cada nó cliente de uma rede. Desta maneira, os clientes utilizam-se de TNAs, enquanto o mapeamento entre os endereços designados e os endereços internos de uma rede é feito dentro das fronteiras da rede.

Embora a UNI não seja um padrão GMPLS, a função de sinalização requerida está muito próxima daquela oferecida pelos protocolos de sinalização RSVP e CR-LDP. Com algumas extensões para suportar características da UNI, tais como endereçamento TNA e identificação de conexão, estes protocolos de sinalização podem ser utilizados para a sinalização UNI. Similarmente, o gerenciamento de vários enlaces UNI pode ser alcançado por extensões do protocolo LMP.

A OIF produziu um documento de especificação denominado UNI - OIF, o qual descreve como os protocolos existentes podem ser estendidos para prover as funcionalidades da interface 
UNI. A UNI - OIF inclui uma faixa de características de protocolos como RSVP, LDP e LMP com extensões UNI, endereçamento UNI TNA e LSPs bidirecionais.

Funcionalmente, o modelo de pares (peer) forma um conjunto do qual o modelo coberto (overlay) é um subconjunto. Isto é, o conjunto de funções requeridas para suportar o modelo coberto (overlay) é um subconjunto das funções requeridas para suportar o modelo de pares (peer). Um modelo coberto (overlay) pode ser derivado de um modelo de pares (peer) pelo bloqueio da distribuição da topologia, preservando todavia, as funções de sinalização da conexão. Esta observação sugere que em vez de utilizar um conjunto de protocolos para suportar o modelo coberto (overlay) e outro para suportar o modelo de pares (peer), um conjunto adequado de protocolos do plano de controle com flexibilidade suficiente para suportar ambos os modelos poderia ser a abordagem mais eficiente.

No Capítulo 5 abordaremos a arquitetura de redes ópticas padronizada pelo ITU-T e como a arquitetura GMPLS pode aí ser inserida. Esta inserção, justifica-se, uma vez que as redes ópticas padronizadas pelo ITU-T (especificadas no Capítulo 5) utilizam um plano de controle, o qual pode ser composto pelos protocolos empregados pelo plano de controle GMPLS.

\subsection{LIMITAÇÕES DA ARQUITETURA GMPLS}

As extensões aos protocolos utilizados na arquitetura GMPLS ainda não estão completamente padronizadas. Neste meio tempo, algumas questões descritas abaixo, demandam atenção e estão sendo objeto de estudo.

1. Segurança

Protocolos IP tradicionais examinam o conteúdo do cabeçalho de um pacote recebido para determinar o próximo nó a envia-lo. Esta operação permite o estabelecimento de programas de segurança de acesso (firewalls). Esta segurança processa-se com a leitura do endereço IP e a posterior autorização de acesso ao conteúdo de uma rede. Em contraste, rótulos GMPLS/MPLS são usados para aumentar a velocidade de processamento (comutação) de um pacote e têm significado local, isto é, os rótulos são somente entendidos e usados internamente entre os dispositivos envolvidos no processamento do referido pacote. Desta maneira, os rótulos não podem ser usados para controlar o acesso à rede com o propósito de prover segurança a esta. Um modo de 
estabelecer segurança em redes GMPLS é por em vigor medidas de segurança durante a configuração da conexão LSP;

2. Sistemas de gerenciamento de rede

O mais importante parâmetro a gerenciar em uma rede IP, por exemplo, a Internet, trata do trabalho executado pela rede na tentativa de transportar o pacote da fonte até seu destino. Em redes GMPLS os sistemas de gerenciamento de rede necessitam controlar algumas centenas de LSPs e seus respectivos status operacionais, rotas e engenharia de tráfego. Este excesso de trabalho requer de um sistema de gerenciamento mais complexo do que aquele utilizado em redes IP;

3. Em redes ópticas o roteamento intra-domínios pode ser realizado com as extensões para os protocolos OSPF e IS-IS. Já para o roteamento interdomínios o IETF não tem uma posição definida e está considerando algumas opções, como por exemplo criar extensões para o protocolo BGP ou estender protocolos como OSPF ou IS-IS para operarem no nível de roteamento entre domínios. Detalhes destas considerações podem ser encontrados em [54]. 


\section{CAPÍTULO 5}

\section{PADRONIZAÇÃO DE REDES ÓPTICAS NO ÂMBITO DO ITU-T E A INSERÇÃO DA ARQUITETURA GMPLS NESTE CONTEXTO}

Neste capítulo será apresentada a arquitetura de redes ópticas desenvolvida pelo ITU-T, discutindo-se as várias recomendações que descrevem tal arquitetura. Finalizando, será explicitada como a arquitetura do plano de controle desenvolvida pelo IETF, o GMPLS, pode ser inserida neste contexto.

\subsection{INTRODUÇÃO}

Existem três instituições desenvolvendo padrões para redes ópticas: ITU-T, OIF e IETF. Enquanto o OIF e o IETF trabalham na padronização de um plano de controle para as redes ópticas, o ITU-T padroniza, através dos Study Groups 13 e 15, não somente o plano de controle como também a arquitetura inteira de uma rede óptica. O trabalho iniciou-se em 1993 focado primeiramente em sistemas SDH amplificados opticamente [55]. Tal empenho ocorreu em resposta à demanda dos membros do ITU-T com referência à necessidade de se criar uma definição completa do modo de operação de uma rede de transporte automaticamente comutada, incluindo gerenciamento, controle e transporte de dados. A padronização visa, desta maneira, facilitar a utilização de equipamentos de diferentes fabricantes e a conexão entre redes.

O conceito de redes ópticas ou ASON (automatically switched optical networks) como é denominada no âmbito do ITU-T, foi desenvolvido com o intuito de transportar vários "clientes" tais como SONET/SDH, Ethernet e ATM, em redes WDM, e gerenciá-los de maneira única. Neste sentido, a característica fundamental de uma rede óptica é o transporte de dados, independentemente dos aspectos relacionados à sua origem (camada cliente). Visando este objetivo o ITU-T desenvolveu uma lista de recomendações, especificadas na Tabela 5.1. Tais recomendações foram classificadas da seguinte forma: 


\section{Aspectos Arquiteturais}

As especificações dos aspectos relacionados à arquitetura de uma rede ASON são determinadas por três recomendações. A recomendação ITU-T G.871 lista quais recomendações especificam a arquitetura de uma rede ASON, bem como as funções por elas implementadas. Uma nova versão desta recomendação será publicada em 2003. Já a recomendação ITU-T G.872 descreve a arquitetura de uma rede ASON propriamente dita. Nela são especificados aspectos relacionados à estruturação das camadas do plano de transporte e a forma como se relacionam com a camada cliente, bem como aspectos relacionados ao gerenciamento da rede. A recomendação ITU-T G 873 será publicada em 2003 e definirá a maneira como as redes ópticas se interconectarão com outras redes. A recomendação G 807 especifica as funções básicas executadas pelo plano de controle. Tais funções serão executadas pelas entidades prospostas na recomendação $G$ 8080. Em adição, a recomendação ITU-T G 8080 especifica o plano de controle da rede ASON, descrevendo aspectos referentes à implantação de conexões comutadas automaticamente. A recomendação previamente denominada G.optperf, prevista para ser publicada em 2003, especificará parâmetros para se avaliar a performance de uma rede óptica;

\section{Estruturas e Mapeamento}

Baseado na arquitetura de rede óptica definida na recomendação ITU-T G.872, a recomendação ITU-T G.709 descreve a maneira de se implementar o mapeamento de tipos diferentes de sinais (SDH, ATM, etc) em uma rede óptica, especificando a funcionalidade dos overheads inseridos para executar a função de gerenciamento deste sinal e a forma de se implementar a multiplexação de tais sinais;

\section{Características Funcionais}

Tendo como base as recomendações ITU-T G.872 e ITU-T G.709, a recomendação ITUT G.798 descreve as características funcionais dos equipamentos presentes em uma rede de transporte óptica. Esta recomendação especifica ambos, os componentes e a metodologia que deve ser utilizada, a fim de se estabelecer as funcionalidades dos elementos presentes em uma rede óptica. Não é especificado individualmente qualquer 
equipamento utilizado em uma rede óptica, e sim o diagrama de funções desejadas, as quais devem ser executadas pelos equipamentos durante as várias etapas do mapeamento do sinal digital (cliente) em um sinal óptico.

\begin{tabular}{|c|c|}
\hline TÓPICO & TÍTULO - RECOMENDAÇÃO PARA REDES ÓPTICAS \\
\hline $\begin{array}{l}\text { Framework para as } \\
\text { Recomendacões }\end{array}$ & $\begin{array}{l}\text { G.871 Framework for Optical Transport Network } \\
\text { Recommendations }[10 / 00]\end{array}$ \\
\hline Aspectos Arquiteturais & $\begin{array}{l}\text { G.872 Architecture of Optical Transport Networks (Publicada em } \\
\text { 02/99 e revisada em 11/01) } \\
\text { G.807 Requirements for the Automatic Switched Transport } \\
\text { Network (ASTN) (Publicada em 07/01) } \\
\text { G.8080 Architecture for the Automatically Switched Optical } \\
\text { Networks (Publicada em 11/01) }\end{array}$ \\
\hline Performance de Erro & $\begin{array}{l}\text { G.optperf Error and availability performance parameters of } \\
\text { Optical Transport Network (OTN) [Previsão 2003] }\end{array}$ \\
\hline $\begin{array}{l}\text { Especificações para o } \\
\text { plano de controle }\end{array}$ & $\begin{array}{l}\text { G.7713 Generalized Call and Connection } \\
\text { Management [12/01] } \\
\text { G.7713.1 Distributed Call and Connection } \\
\text { Management-PNNI Implementation (Previsão 2003) } \\
\text { G.7713.2 Distributed Call and Connection } \\
\text { Management-GMPLS RSVP-TE Implementation (Previsão 2003) } \\
\text { G.7713.3 Distributed Call and Connection } \\
\text { Management-GMPLS CR-LDP Implementation (Previsão 2003) } \\
\text { G.7714 Generalized automatic discovery techniques } \\
\text { [10/01] } \\
\text { G.7715 Architecture and requirements for routing in } \\
\text { automatically switched optical networks [06/2002] }\end{array}$ \\
\hline $\begin{array}{l}\text { Aspectos de } \\
\text { Gerenciamento }\end{array}$ & $\begin{array}{l}\text { G.874 Management aspects of the optical transport network } \\
\text { element [11/01] } \\
\text { G.874.1 Optical Transport Network (OTN) Protocol-Neutral } \\
\text { Management Information Model For The Network Element View } \\
\text { [01/02] } \\
\text { G.875 Optical Transport Network (OTN) management } \\
\text { information model for the network element view (Previsão 2003) }\end{array}$ \\
\hline Estruturas e Mapeamento & G.709 Interface for the optical transport network (OTN) [02/01] \\
\hline Características Funcionais & $\begin{array}{l}\text { G.798 Characteristics of optical transport network (OTN) } \\
\text { equipment functional blocks [11/01] }\end{array}$ \\
\hline $\begin{array}{l}\text { Performance de Jitter e } \\
\text { Wander }\end{array}$ & $\begin{array}{l}\text { G.8251 (G.otnjit) The control of jitter and wander within the } \\
\text { optical transport network (OTN) [11/01] }\end{array}$ \\
\hline Aspectos da Camada Física & $\begin{array}{l}\text { G.694.1 Spectral grids for WDM applications: DWDM frequency } \\
\text { grid [06/2002] } \\
\text { G.694.2 Spectral grids for WDM applications: CWDM } \\
\text { wavelength grid [06/2002] } \\
\text { G.664 General Automatic Power Shut-Down Procedures for } \\
\text { Optical Transport Systems [09/99] }\end{array}$ \\
\hline
\end{tabular}




\begin{tabular}{|l|l|}
\hline & G.959.1 Optical Transport Networking Physical Layer Interfaces \\
& {$[02 / 01]$} \\
& G.693 Optical interfaces for intra-office systems [11/01] \\
Sup.dsn Optical System Design [Previsão 2003]
\end{tabular}

TABELA 5.1: Recomendações que especificam a rede de transporte óptica (ASON) padronizada pelo ITU-T. As recomendações escritas na cor preta especificam a arquitetura da rede, na cor azul definem o plano de controle, na cor verde estabelecem o plano de gerenciamento e na cor vermelha implementam o plano de transporte.

Para atingir este objetivo são especificados um conjunto de blocos de funções básicas e um conjunto de regras pelas quais tais funções possam ser combinadas a fim de descrever os equipamentos usados em uma rede de transporte óptica. Este conjunto compreende as funções básicas necessárias para especificar completamente uma estrutura funcional genérica de uma rede de transporte óptica. A fim de estar de acordo com esta recomendação, a funcionalidade de qualquer equipamento que será empregado no plano de transporte de uma rede de transporte óptica deve ser descrita como uma interconexão de um conjunto de blocos de funções básicas contidas nesta recomendação, obedecendo-se regras estabelecidas. Por exemplo, o processamento do overhead em cada camada de uma rede de transporte óptica, necessário para operações de gerenciamento dos sinais digitais, é definido como uma função básica, que todos os equipamentos que processam este sinal devem implementar. 


\section{Aspectos de Gerenciamento}

A recomendação ITU-T G.874 implementa aspectos de gerenciamento de uma rede de transporte óptica. O gerenciamento da camada óptica é separado do gerenciamento da camada cliente para que os meios de gerenciamentos possam ser utilizados independentemente dos sinais dos clientes. A recomendação ITU-T G.874.1 provê um modelo de gerenciamento de informações para gerenciar os elementos de rede. $\mathrm{O}$ modelo descreve as entidades gerenciadas e suas propriedades, que são úteis para descrever a troca de informações necessárias ao gerenciamento dos elementos da rede. Esta recomendação deverá ser utilizada como base para a definição de um modelo de gerenciamento de informações para um protocolo específico, como por exemplo, o protocolo SNMP (simple network management protocol). Mapear entidades neutras em relação a protocolos em objetos específicos de um protocolo é uma decisão própria de cada protocolo utilizado e deverá ser descrita em uma nova recomendação. Seguindo esta tendência a recomendação ITU-T G.875 deverá ser publicada em 2003.

\section{Aspectos da Camada Física}

A recomendação ITU-T G.959.1 especifica um padrão de interconexão, ou seja, as interfaces físicas para compatibilidade entre redes de diferentes fornecedores. As especificações são organizadas de acordo com os tipos de fibras ópticas empregadas, a distância do enlace entre nós, o tipo de codificação utilizado e a taxa de bit do canal óptico (2,5 e $10 \mathrm{Gbps}$, sendo que o valor de $40 \mathrm{Gbps}$ está em estudo). As interfaces são especificadas para sistemas com canal óptico único e para sistemas DWDM. São especificados, também, os parâmetros físicos destas interfaces, tais como a máxima dispersão cromática e a máxima atenuação entre nós. Adicionalmente, uma nova recomendação, a ser publicada em 2003, provisoriamente denominada G.dsn, implementará aspectos relacionados ao projeto de redes ópticas para ambientes de redes LAN (local area network), WAN (wide area network) e de longa distância utilizando canal óptico único e sistemas DWDM.

As recomendações ITU-T G.694.1 e 694.2 especificam o espaçamento entre canais utilizados em sistemas DWDM e CWDM (coarse wavelength division multiplexing) 
respectivamente. Sistemas CWDM podem ser usados em redes metropolitanas, sendo caracterizados por utilizar um espaçamento entre canais maior do que os sistemas DWDM, da ordem de $20 \mathrm{~nm}$. A primeira recomendação, como mencionado no Capítulo 1, implementa a padronização de uma grade de freqüências centrada em 193,1 THz $(1552,52 \mathrm{~nm})$, a qual suporta espaçamento entre canais de 12,5, 25, 50 e $100 \mathrm{GHz}$. Como exemplo, em uma fibra com espaçamento entre canais de $50 \mathrm{GHz}$, as freqüências permitidas são definidas por $193,1+\mathrm{n} \times 0,05$, onde $\mathrm{n}$ é um número inteiro positivo ou negativo, incluindo o 0 . A segunda recomendação implementa a padronização de uma grade de comprimentos de onda variando de 1270 a $1610 \mathrm{~nm}$ com espaçamento de 20 $\mathrm{nm}$.

A padronização para interfaces utilizadas na interligação de redes com enlaces de até 2 $\mathrm{km}$ de distância utilizando fibras óticas com apenas um canal óptico, o qual pode apresentar uma taxa de transmissão de bits de 10 ou 40 Gbps, é estabelecida na recomendação ITU-T G.693.

A recomendação ITU-T G.664 estabelece procedimentos para garantir condições de trabalho seguro nas interfaces ópticas de uma rede. Esta recomendação define procedimentos para a interrupção automática da emissão de laser (ALS-automatic laser shutdown) e redução automática de potência (APR-automatic power reduction) nas interfaces ópticas quando os limites de segurança ópticos são excedidos.

As recomendações ITU-T 661, 662, 663 e 671 especificam os componentes de uma rede óptica. As três primeiras especificam os amplificadores ópticos e a última os demais equipamentos utilizados em um rede óptica. A recomendação ITU-T 661 define os parâmetros e seus respectivos métodos de teste para os amplificadores ópticos (EDFAs). Como exemplo, o parâmetro ganho de pequenos sinais (SSG-small-signal gain) é definido como o ganho do amplificador quando este opera no regime linear. A recomendação ITU-T 662 estabelece as características dos amplificadores ópticos. Tais características dependem da forma de utilização destes amplificadores, sendo subdivididas em três categorias: amplificadores de potência, pré-amplificadores e amplificadores de linha. É definida também uma lista composta pelos parâmetros que especificam cada um dos três tipos de amplificadores quando empregados em sistemas com canal óptico único ou sistemas DWDM. A recomendação ITU-T 663 determina os valores dos parâmetros listados na recomendação anterior, mas apenas para aplicações empregando canal óptico único. Os valores para os parâmetros empregados em sistemas 
DWDM estão ainda em estudo. Também são definidos os fatores que limitam a transmissão de um sinal óptico em uma fibra óptica, os quais podem influir no desempenho de um amplificador óptico. Métodos para minimizar a influência destes fatores são apresentados.

A recomendação ITU-T 671 define os demais componentes de uma rede óptica como, por exemplo, filtros ópticos fixos ou sintonizáveis, isoladores ópticos, atenuadores ópticos, emendas ópticas, terminações ópticas, e outros. Para cada um dos componentes são definidos os parâmetros que o caracterizam com seus respectivos valores. Por exemplo, em relação às emendas ópticas e outros dispositivos passivos, um dos parâmetros definidos é a perda de inserção, isto é, a redução na potência óptica entre as portas de entrada e de saída de um componente. Este parâmetro é definido por:

$$
I L=-10 \log \left(\frac{P_{\text {out }}}{P_{\text {in }}}\right)_{\mathrm{dB}}
$$

A potência $\mathrm{P}_{\text {in }}$ é a potência medida na porta de entrada e $\mathrm{P}_{\text {out }}$ é a potência recebida na porta de saída. A definição de OADMs e os valores dos parâmetros que o caracterizam não fazem parte desta recomendação e serão incluídas em versões posteriores. As fibras ópticas são especificadas pelas recomendações ITU-T G 651, 652, 653, 654 e 655.

Como demonstrado pelas recomendações listadas acima, o padrão ASON (automatically switched optical networks) não é um protocolo ou uma coleção deles. É sim, uma arquitetura (Figura 5.1) composta de um plano de controle, um plano de gerenciamento e um plano de transporte. A padronização de cada um deles é estabelecida pelas recomendações listadas na Tabela 5.1. Assim, as recomendações escritas na cor preta especificam a arquitetura da rede, na cor azul definem o plano de controle, na cor verde estabelecem o plano de gerenciamento e na cor vermelha implementam o plano de transporte.

Como muitos projetos de padronização executados pelo ITU-T, a arquitetura ASON foi (e continua sendo) desenvolvida em um modo top-down. Começando com uma completa lista de requerimentos e movendo-se de uma visão de alto nível da arquitetura em direção à especificação dos componentes individuais. Somente quando um componente é definido em detalhes, é que os protocolos são anexados à arquitetura. Qualquer protocolo que preencha os requisitos dos componentes da arquitetura pode potencialmente conseguir do ITU-T o selo de aprovação. Deve-se notar que as recomendações listadas na Figura 5.1 são desenvolvidas usando 
uma abordagem por fases, dependendo da maturidade da tecnologia envolvida e das necessidades do mercado. Na fase 1, na qual está a maioria delas, dá-se ênfase a sistemas ponto a ponto e na fase 2 a ênfase será dada à criação de extensões para a implantação de OADMs e OXCs. As recomendações ITU-T 871, 872 e 959.1 não estão sujeitas a esta abordagem por fases.

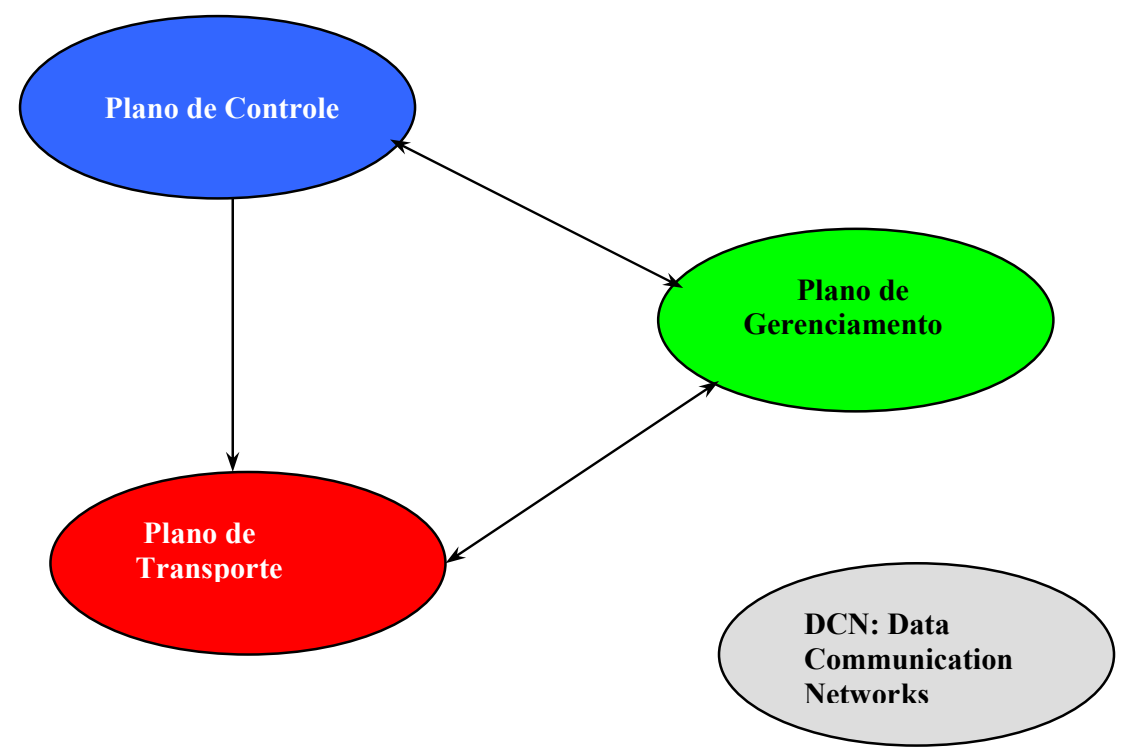

FIGURA 5.1: Relacionamento entre os planos da arquitetura ASON [56]. As DCNs são utilizadas com o intuito de formar uma rede de comunicação, onde são trocadas as informações de gerenciamento e ou sinalização entre os diversos planos.

Em linhas gerais, o plano de transporte forma uma rede para os dados do usuário. O plano de gerenciamento monitora e administra o plano de transporte. O plano de controle provê a configuração e extinção de circuitos (caminhos ou rotas) através de sinalização distribuída, a qual habilita novos serviços de rede (BOD-bandwidth on demand).

A seguir na Seção 5.2 apresentar-se-á o plano de controle ASON. Na Seção 5.3 estará especificada a maneira de se incorporar a arquitetura GMPLS à padronização ASON. Nas Seções 5.4 e 5.5 são apresentados o plano de transporte e o plano de gerenciamento. O capítulo é finalizado na Seção 5.6, na qual os pontos mais importantes são resumidos. 


\subsection{PLANO DE CONTROLE ASON}

O plano de controle em uma rede ASON tem a função de estabelecer conexões por meio de sinalização automática, evitando a operação manual da rede. As recomendações que especificam o plano de controle no âmbito do ITU-T são apresentadas na Figura 5.2.

A arquitetura do plano de controle é descrita na recomendação ITU-T G 8080. Nesta recomendação são definidos os componentes presentes no plano de controle ASON e a forma de configuração de chamadas e conexões. A recomendação ITU-T G 7713 cobre aspectos associados ao plano de controle ASON, estabelecendo os requisitos de sinalização necessários para a comunicação dos componentes do plano (apresentados na seção seguinte). Esta recomendação especifica operações para a configuração de chamadas que estabelecem uma conexão. Chamadas suportando múltiplas conexões serão objeto de futuras recomendações. A abordagem desta recomendação não define nenhum protocolo específico. As recomendações ITU-T G 7713.1, G 7713.2, G 7713.3 estão ainda em discussão com previsão de publicação para o biênio 2003/2004 e implementarão diferentes protocolos para a execução das funções de sinalização.

A fim de prover mecanismos para o estabelecimento de chamadas e conexões de uma maneira automática, é necessário o conhecimento dos recursos presentes na rede óptica. Estes recursos podem ser estabelecidos manualmente ou descobertos automaticamente. A descoberta automática da topologia e dos recursos é o assunto da recomendação ITU-T G 7714. Esta recomendação não implementa nenhum protocolo de descoberta automática de recursos, apenas caracteriza de uma maneira geral a troca de mensagens para determinar a existência de conectividade entre dois elementos. Por exemplo, a mensagem HELLO deverá ser utilizada sobre os enlaces para a detecção de conectividade. Futuras recomendações (ITU-T G7714.1, G7714.2) implementarão os protocolos que realizarão a função de descoberta automática de recursos. A recomendação ITU-T G 7715 especifica os requisitos e a arquitetura das funções de roteamento utilizadas para a configuração de conexões em uma rede ASON.

A arquitetura de roteamento estabelece uma segregação hierárquica de roteamento, ou seja, a topologia de uma rede ASON é descrita por domínios, visando tornar tais redes escaláveis. Tais domínios podem ser particionados em áreas de roteamento, as quais tornam o processo de roteamento mais racional. 


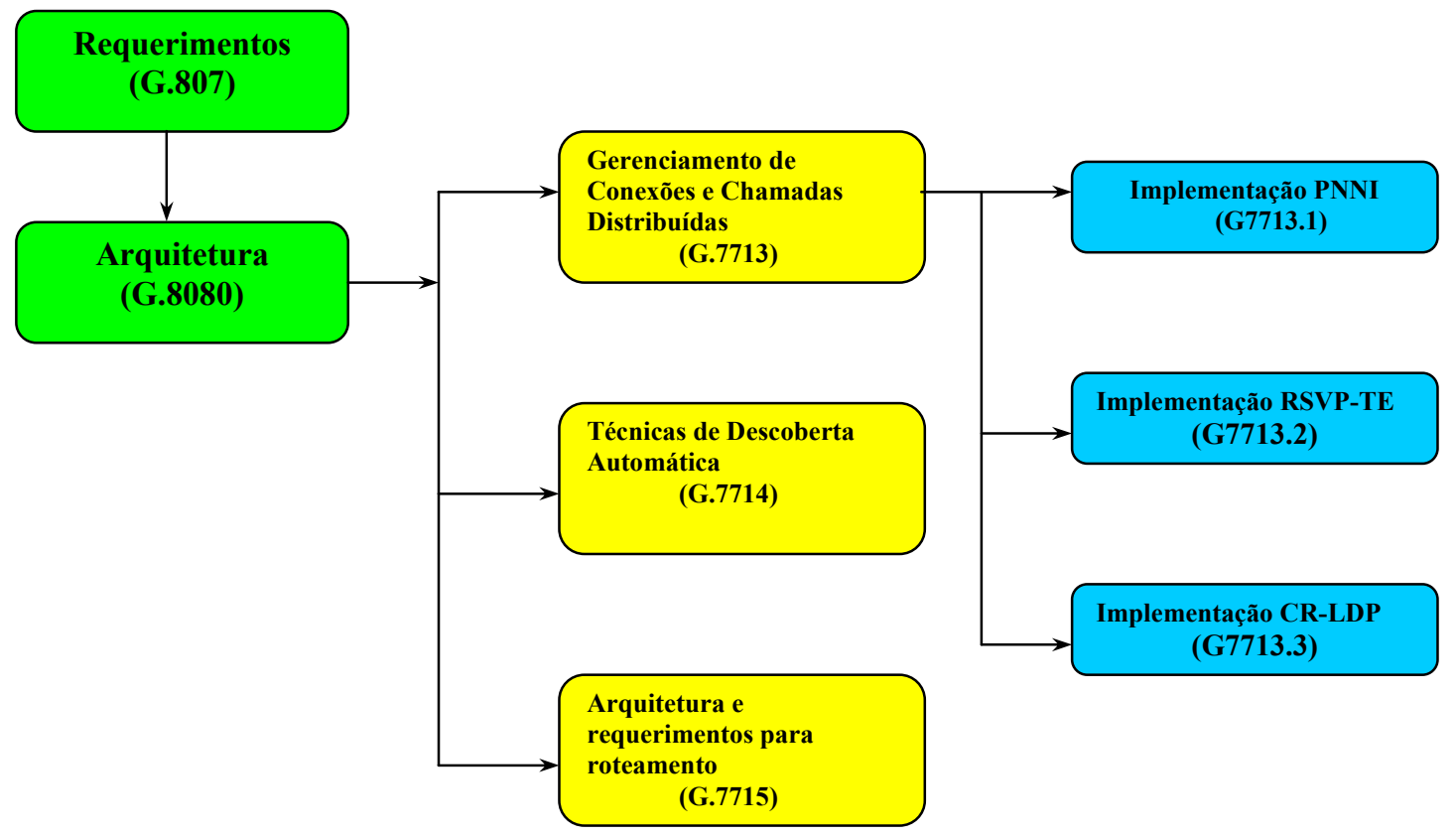

ASTN: Automatic Switched Transport Network PNNI: Private Network-Private Interface

FIGURA 5.2: Recomendações que descrevem o plano de controle ASON. As recomendações em azul serão publicadas no biênio 2003/2004.

Em outras palavras, dentro destas áreas de roteamento são trocadas informações de roteamento locais e informações menos detalhadas sobre áreas de roteamento remotas. Áreas de roteamento podem ser particionadas recursivamente, criando uma hierarquia de informações de roteamento. A recomendação ITU-T G 7715 estabelece os blocos funcionais que serão utilizados para as funções de roteamento em redes ASON, não especificando qualquer tipo de protocolo. Futuras recomendações (por exemplo, G 7715.1, G7715.2) implementarão protocolos específicos para o roteamento em redes ASON.

Enquanto o GMPLS configura um caminho (path) entre dois usuários, o plano de controle ASON utiliza-se dos conceitos de chamada e conexão para realizar tal função. A sinalização para permitir uma chamada é estabelecida no ingresso ou egresso da rede ou nas fronteiras entre domínios (através das interfaces UNI - user network interface e E-NNI - exterior node to node interface, apresentadas na próxima seção). 


\subsubsection{Arquitetura do Plano de Controle ASON}

A Figura 5.3 apresenta os componentes do plano de controle ASON.

\begin{tabular}{|c|c|c|}
\hline $\begin{array}{l}\text { TP-Policiamento de } \\
\text { tráfego } \\
\text { (Traffic Policing) }\end{array}$ & $\begin{array}{l}\text { CallC- Controlador de } \\
\text { chamadas } \\
\text { (Call controller) }\end{array}$ & $\begin{array}{l}\text { PC- Controlador de } \\
\text { Protocolo } \\
\text { (Protocol controller) }\end{array}$ \\
\hline \multicolumn{3}{|c|}{ PLANO DE CONTROLE - ASON } \\
\hline $\begin{array}{l}\text { CC-Controlador de } \\
\text { Conexão } \\
\text { (connection controller) }\end{array}$ & $\begin{array}{l}\text { RC-Controlador de } \\
\text { Roteamento } \\
\text { (routing controller) }\end{array}$ & $\begin{array}{l}\text { LRM-Gerenciador de } \\
\text { Recursos de enlace } \\
\text { (link resource manager) }\end{array}$ \\
\hline
\end{tabular}

FIGURA 5.3: Componentes da arquitetura do Plano de Controle ASON.

A função de cada componente é descrita abaixo:

1. Controlador de Conexão (CC)

O componente controlador de conexão é responsável pela coordenação entre o gerenciador de recursos de enlace (LRM-link resource manager) e o controlador de roteamento (RC-routing controller) com o propósito de gerenciar e supervisionar a configuração de uma conexão;

2. Controlador de Roteamento (RC)

O papel do controlador de roteamento é responder aos pedidos do controlador de conexão (CC) referentes às informações que habilitem estabelecer rotas em domínios sobre sua responsabilidade. Tais informações são necessárias para a configuração de uma conexão;

3. Gerenciador de Recursos de enlace (LRM)

O LRM é responsável pelo gerenciamento de enlaces incluindo alocação de recursos, informação de status do referido enlace e a atual topologia da rede, com a indicação de quais recursos estão sendo utilizados. O LRM informa ao RC qualquer mudança de estado dos recursos dos enlaces que estão sob seu controle; 
4. Policiamento de tráfego (TP)

O papel do componente responsável pelo policiamento de tráfego é checar se o usuário de uma referida conexão esta enviando tráfego de acordo com os parâmetros acordados (como, por exemplo, a taxa de transmissão). O TP será especificado em detalhes em recomendações posteriores;

5. Controlador de chamadas (CallC)

As chamadas são controladas por dois tipos de controladores de chamada, cuja função é gerar e processar pedidos de chamadas;

a) Controlador de chamadas das partes chamante e chamada (calling/called party call controller);

b) Controlador de chamadas de rede (network call controller);

O controlador da parte chamante (calling party call) interage com o controlador da parte chamada (called party call controller) por meio de um ou mais controladores de chamada de rede (network call controller) intermediários. A Figura 5.4 apresenta um modo de operação dos controladores CallC;

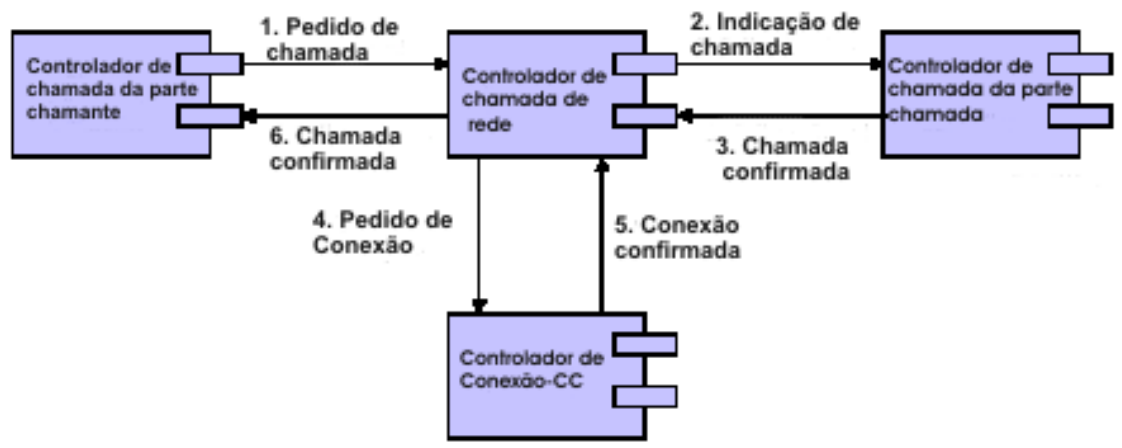

FIGURA 5.4: Interação entre os controladores da parte chamada/chamante e o controlador de chamada de rede. 
6. Controlador de Protocolo (PC)

O controlador de protocolo executa a função de mapear os parâmetros das interfaces dos componentes do plano de controle em mensagens que são transportados pelos protocolos que suportam a interconexão. A Figura 5.5 mostra a utilização de um PC pelos controladores CC e LRM;

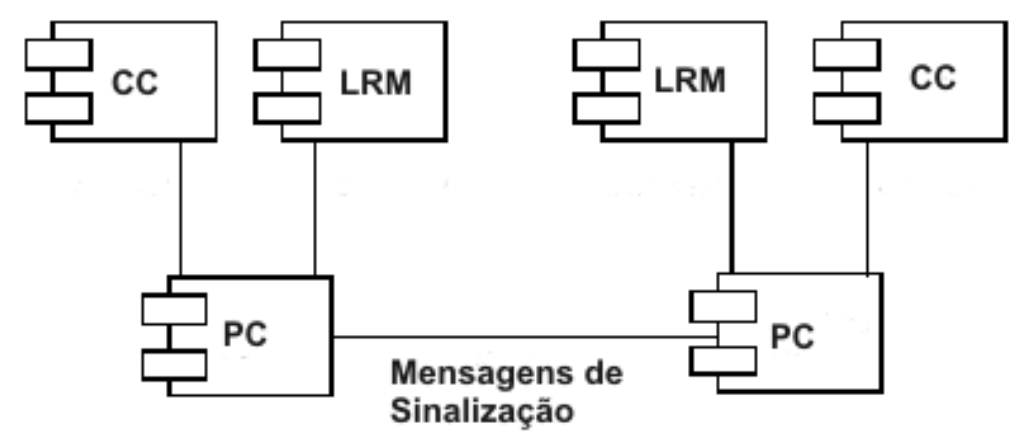

FIGURA 5.5: Controladores CC e LRM utilizando um controlador de protocolo PC.

A recomendação ITU-T G 8080 apresenta detalhes da interação entre os componentes de um plano de controle para a configuração de uma conexão.

\subsubsection{Pontos de Referência ASON}

O plano de controle ASON vê a rede como composta de domínios, os quais interagem com outros domínios em um modo padronizado. Tais domínios podem ser divididos, por exemplo, em domínios geográficos ou domínios com o mesmo tipo de equipamento. As interfaces entre domínios (Figura 5.6) são conhecidas como pontos de referência, sendo denominados: UNI (user network interface), E-NNI (exterior node to node interface) e I-NNI (interior node to node interface). Estes pontos de referências indicam as localizações nas quais mensagens de sinalização e roteamento são trocadas utilizando os protocolos padronizados.

Os pontos de referência do plano de controle ASON são assim definidos:

1. User network interface: UNI define uma interface entre o cliente e a rede. Ao cliente é permitido executar diversas funções incluindo:

a. Criar, modificar e encerrar chamadas e conexões;

b. Inquirir o status de uma conexão já existente (se está ou não ativa); 
Obs: Não existe função de roteamento associada com a interface UNI;

2. Exterior node to node interface: O E-NNI define uma interface entre domínios diferentes e pode ser visto como similar à uma interface UNI com função de roteamento que permita a troca de informações sobre o estabelecimento de rotas entre diferentes domínios;

3. Interior node to node interface: I-NNI define uma interface entre elementos de rede de um mesmo domínio. Em outras palavras, o I-NNI define uma interface entre CCs (Controladores de Conexão) pertencentes a um mesmo domínio;

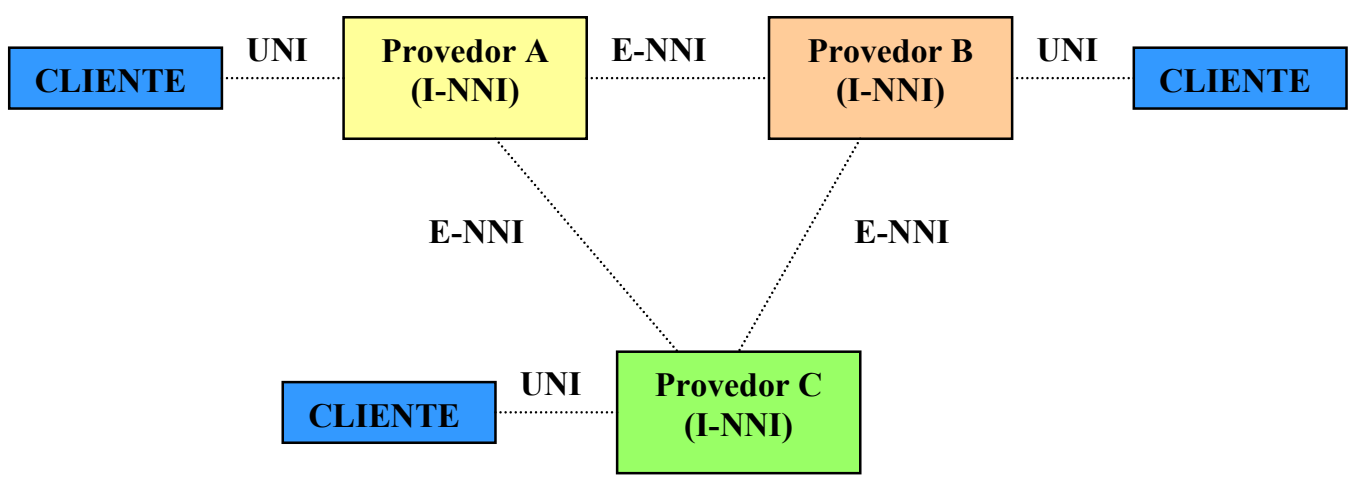

UNI - User Network Interface

E-NNI - Exterior Node to Node Interface

I-NNI - Interior Node to Node Interface

FIGURA 5.6: Rede ASON mostrando a divisão de domínios e os pontos de referências UNI, E-NNI e I-NNI.

\subsubsection{Rede de Comunicação de Dados-DCN (Data Communication Network)}

Similar à arquitetura GMPLS, que utiliza canais de controle para a comunicação de mensagens de sinalização e roteamento, a arquitetura ASON especifica, por meio da recomendação ITU-T G 7712, uma rede de comunicação de dados onde são trocadas as informações de gerenciamento, sinalização e roteamento entre os elementos de uma rede. Por exemplo, as informações de gerenciamento requerem uma rede de comunicação, a qual é denominada rede de comunicação de gerenciamento (MCN-management communication network), para transportar mensagens de gerenciamento entre os vários componentes de um 
sistema gerenciável. Adicionalmente, o plano de controle ASON requer uma rede de comunicação para o transporte de mensagens de sinalização entre os componentes do plano de controle (por exemplo, entre os CCs). Tal rede é denominada rede de comunicação de sinalização (SCN-Signalling Communication Network). Várias tecnologias de transporte de dados podem ser empregadas para a confecção de uma rede DCN, como por exemplo, LAN, ATM, SDH.

\subsection{PROTOCOLOS GMPLS E PONTOS DE REFERÊNCIA ASON}

Enquanto o GMPLS foi desenvolvido por uma comunidade fortemente associada às redes de dados baseadas em IP, os membros do ITU-T são provenientes de uma comunidade associada com o setor de telecomunicações. Assim, enquanto o GMPLS herda conceitos e protocolos IP, o ASON aproxima-se de conceitos e protocolos usados em redes de transporte de telecomunicações, tais como SONET/SDH e ATM. Os encontros dos grupos de estudos do ITUT são realizados em intervalos de nove meses enquanto que os do IETF são realizados em intervalos de quatro meses. Detalhes do encontro do ITU-T realizado no mês de maio de 2002, bem como os membros participantes podem ser encontrados em [57].

O plano de controle GMPLS descrito no capítulo anterior refere-se a um conjunto de protocolos que, quando completado, poderá prover interoperabilidade fim a fim dos dispositivos em redes ópticas.

Os protocolos são os seguintes:

a) Generalizad RSVP-TE para sinalização;

b) Generalizad CR-LDP para sinalização;

c) OSPF com extensões TE (Traffic Engineering) para roteamento intra-área;

d) IS-IS com extensões TE (Traffic Engineering) para roteamento intra-área;

e) LMP para realizar o gerenciamento de enlaces e implementar funções de descobertas;

Os protocolos RSVP-TE e CR-LDP são protocolos de sinalização que, como visto no capítulo anterior, desempenham efetivamente funções idênticas. Foram implementados devido a conflitos de interesses de seus propositores, presentes entre os membros de IETF. Os protocolos OSPF-TE e IS-IS são também equivalentes. Entretanto, a presença de ambos tem por base razões históricas e não políticas [58]. Protocolos de roteamento interáreas para o ambiente óptico não 
foram definidos até este momento. Como discutido no capítulo anterior, o IETF está considerando algumas opções.

Por outro lado, o ITU implementará na arquitetura do plano de controle ASON apresentada na Figura 5.14 recomendações que incorporarão protocolos presentes na arquitetura GMPLS (RSVP-TE e CR-LDP), podendo se constatar a existência de vários protocolos de sinalização para executar a mesma função. Tais protocolos serão empregados nos pontos de referência já mencionados.

O ponto de referência UNI assegura aos clientes ASON a criação de conexões, isto é, permite a estes sinalizarem para a rede com o intuito de criarem novas conexões com atributos específicos. Tais atributos poderiam incluir largura de banda, proteção e restauração. Além do mais, pode-se interromper ou modificar uma conexão. Como visto no capítulo anterior, a OIF (Optical Internetworking Forum) tem trabalhado na especificação da interface UNI (User Network Interface 1.0 - Signaling Specification- OIF-UNI-1.0). Esta especificação, fortemente influenciada pelo ITU, busca fundir os requisitos do plano de controle ASON com o perfil de vários protocolos GMPLS (RSVP-TE, CR-LDP e LMP). Formas de se implementar extensões para incluir suporte a chamadas, no âmbito do IETF, para os protocolos RSVP-TE e CR-LDP podem ser encontradas em [59] e [60] respectivamente.

Segundo o IETF [61], para o ponto de referência I-NNI poder-se-iam utilizar os protocolos de roteamento OSPF ou IS-IS. Já para o ponto de referência E-NNI, o IETF não tem uma posição definida e está considerando algumas opções como, por exemplo, criar extensões para o protocolo BGP ou estender protocolos como OSPF ou IS-IS para operarem no nível de roteamento entre domínios.

O ITU não definiu quais protocolos de roteamento utilizará nos referidos pontos de referência. Outras opções além daquelas propostas pelo IETF estão em estudo. Por exemplo, o OIF estendeu no início de 2002 o OSPF-TE para trabalhar como um protocolo hierárquico de roteamento de estado de enlace, conhecido como DDRP (domain to domain routing protocol).

\subsection{PLANO DE TRANSPORTE}

Como mencionado anteriormente, a característica fundamental de uma rede óptica é transportar dados de forma transparente, independentemente dos aspectos relacionados à sua origem. Para tal, o plano de transporte é disposto em camadas, especificadas na recomendação 
ITU-T G 709 [62] e apresentadas na Figura 5.7 (b), sendo descritas como: OCh (Optical Channel), OMS (Optical Multiplex Section), OTS (Optical Transmission Section).

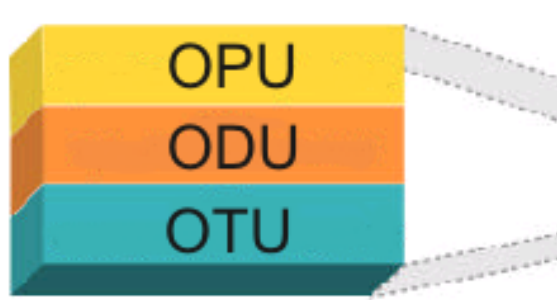

a-)

OCh: optical channel

OMS: optical multiplex section

OTS: optical transmission section

OPU: optical channel payload unit

ODU: optical channel data unit

OUT: optical transport unit
IP ATM Ethernet STM-N

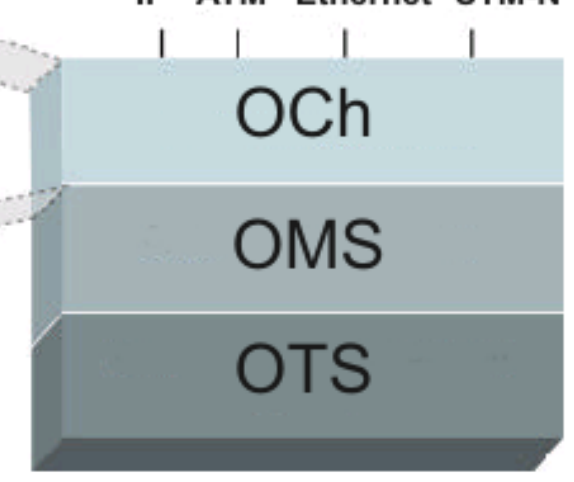

b-)

FIGURA 5.7: a) Subcamadas do plano de transporte; b) Camadas do Plano de transporte.

Sinais como SONET/SDH, Ethernet, IP e ATM são mapeados do formato digital para o formato óptico na camada OCh. Esta é subdividida em três subcamadas, como mostrado na Figura 5.7 (a). As funções de cada subcamada serão especificadas nas seções seguintes. Os canais presentes na camada OCh são multiplexados pela camada OMS. Tais canais são transportados opticamente em um segmento de fibra óptica pela camada OTS. A Figura 5.8 ilustra como estas camadas apresentam-se em um enlace de uma rede óptica.

A estrutura destas camadas é similar às subcamadas de seção, linha e caminho da arquitetura SONET/SDH apresentada na Figura 2.4 do Capítulo 2.

As camadas do plano de transporte podem ser definidas como :

1. Camada OCh: Esta camada provê o transporte fim a fim de canais ópticos para transmitir de forma transparente informações de clientes com diferentes tipos de formatos (SDH, ATM, IP). Esta camada gerencia individualmente os canais ópticos. 


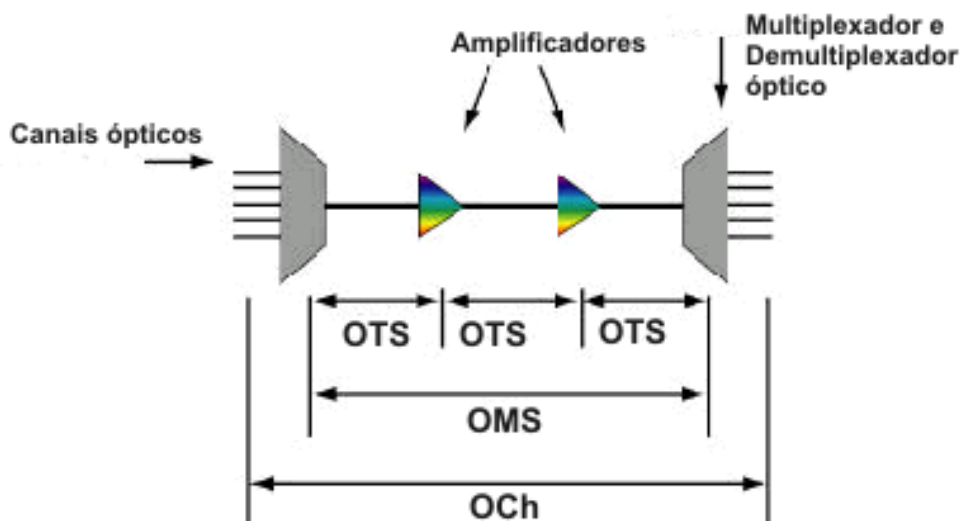

OCh: optical channel

OMS: optical multiplex section

OTS: optical transmission section

FIGURA 5.8: Camadas do plano de transporte em um enlace de uma rede óptica.

2. Camada OMS: Esta camada provê funcionalidades para o transporte de um sinal óptico com múltiplos comprimentos de onda, ou seja, esta camada executa a função de multiplexar e gerenciar um conjunto composto de vários comprimentos de onda.

3. Camada OTS: Esta camada provê funcionalidades para a transmissão do sinal óptico em meios ópticos de vários tipos (diferentes tipos de fibras ópticas). Ou seja, executa o transporte de um feixe composto de vários comprimentos de onda provenientes da camada OMS. As recomendações, que especificam os tipos de fibras ópticas padronizadas constam do item Fibras Ópticas apresentado na Tabela 5.1.

A camada OCh fornece, como visto acima, um sinal óptico para a camada OMS independente do formato digital do sinal carregado pela portadora óptica, que é o interesse, de fato, da camada OMS. O canal óptico pode então ser multiplexado com outros canais ópticos resultando em um sinal OTM-n e adaptado à diferentes tipos de fibras ópticas pela camada OTS. Portanto, as funções de gerenciamento de um canal óptico são independentes do sinal digital que lhe deu origem. Em outras palavras, não é possível analisar um sinal digital por meio do comprimento de onda no qual o referido sinal digital foi mapeado. Assim, a camada OCh foi subdividida em outras três subcamadas (OPU-optical channel payload unit, ODU-optical channel data unit e OTU-optical transport unit). O objetivo de cada subcamada é facilitar o gerenciamento do sinal digital por meio do acréscimo de overheads ao campo de carga (payload) 
deste. No entanto, estas subcamadas são acessíveis apenas nos pontos onde o sinal óptico é transladado para o sinal digital, ou seja, onde ocorre a conversão eletroóptica do sinal. Desta maneira, como especificado na recomendação ITU-T G 959.1 [63] as interfaces interdomínios IrDI (inter-domain interface) são os pontos onde o sinal óptico é convertido para o sinal digital, constituindo, assim, em pontos de regeneração [64]. Tais interfaces conectam redes ópticas de diferentes operadores, os quais podem usar equipamentos de diferentes fornecedores. A padronização desta interface torna-se de fundamental importância para a perfeita compatibilidade entre redes ópticas de diferentes operadores. Em síntese, as subcamadas OPU, ODU e OTU permitem o gerenciamento do sinal digital aproveitando-se da presença de pontos de regeneração do sinal, onde ocorre a conversão eletroóptica do sinal.

\subsubsection{Formato do quadro especificado na Recomendação ITU-T G 709 para a camada OCh}

A Figura 5.9 (a) mostra a estrutura de quadro formada pela camada OCh, ou seja, a estrutura a ser inserida em um canal óptico.

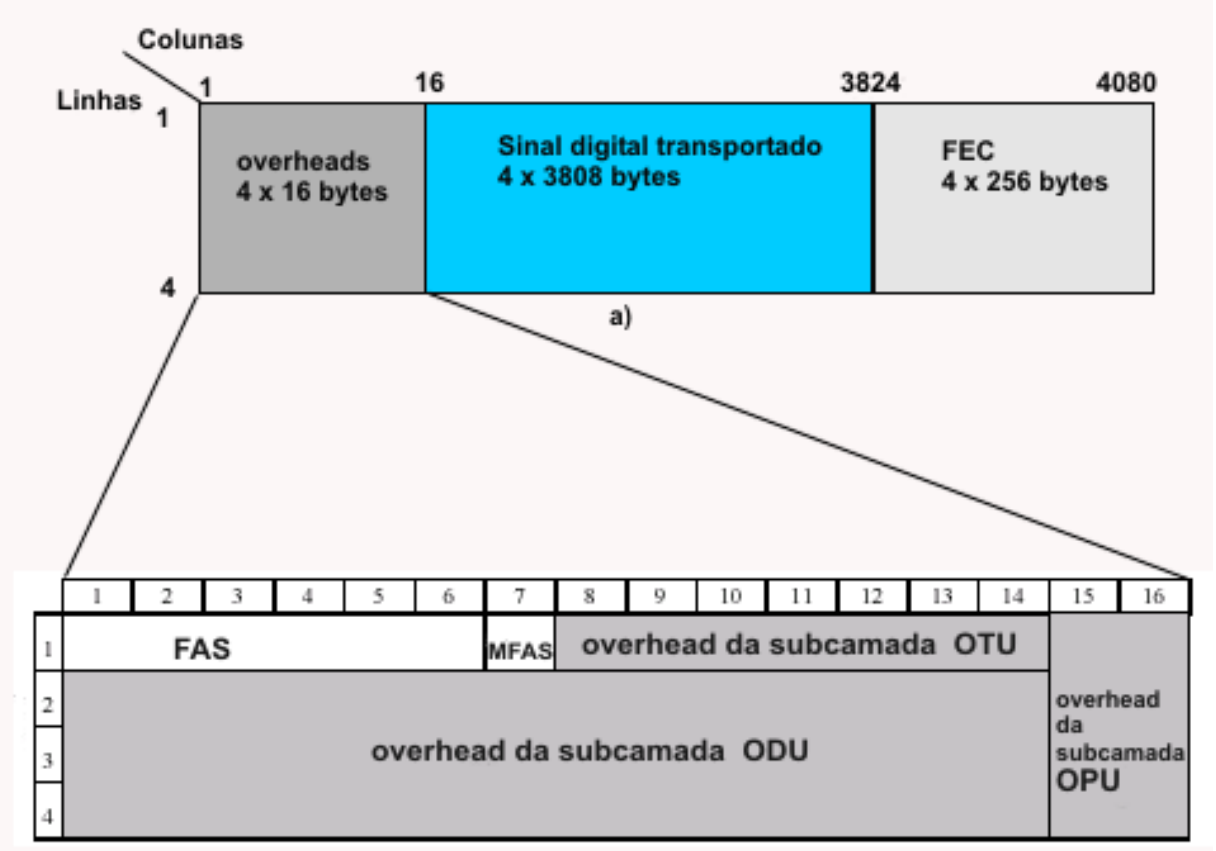

b)

FEC - forward error correction FAS - frame alignment signal

MFAS - multiframe alignment signal
OPU: optical channel payload unit ODU: optical channel data unit OUT: ontical transport unit

FIGURA 5.9: a) Estrutura de quadro utilizada em um canal óptico; b) Detalhe do overhead introduzido pelas camadas OPU, ODU e OTU. 
Este quadro é enviado em diferentes intervalos de tempo, correspondendo às três velocidades de transmissão especificadas (Tabela 5.2).

\begin{tabular}{|c|l|l|}
\hline $\begin{array}{c}\text { Taxa de Transmissão } \\
\mathbf{4 0 8 0} \mathbf{x} \mathbf{4} \text { bytes } \\
\text { Gbps }\end{array}$ & Período $(\boldsymbol{\mu s})$ & Freqüência Nominal (Kbits/s) \\
\hline 2,5 & 48,971 & 2666057,143 \\
\hline 10 & 12,191 & 10709225,316 \\
\hline 40 & 3,035 & 43018413,559 \\
\hline
\end{tabular}

TABELA 5.2: Tempo de transmissão do quadro formado pela camada OCh [56].

O quadro básico é formado por uma estrutura de bytes com 4080 colunas e 4 linhas. A ordem de transmissão é da esquerda para a direita e de cima para baixo. Dentro de cada byte o bit mais significante é transmitido primeiro. O overhead (Figura 5.9 (b)) introduzido pelas camadas OPU, ODU e OTU é composto de 4 linhas por 16 colunas. A FEC (forward error correction), composta de 4 linhas por 256 colunas, é introduzida pela camada OTU com a intenção de habilitar a detecção e correção de erros causados pelo meio de transmissão. Se o processo de FEC não for utilizado, os bytes designados para esta função serão codificados com 0 . Detalhes do processo de cálculo para a FEC são encontrados em [56].

Os 6 primeiros bytes denominados FAS (frame alignment signal) são utilizados para a detecção do quadro e constam de uma seqüência de 3 de bytes "1111 0110" e 3 bytes "0010 1000". O byte MFAS (multiframe alignment signal) possui a função de detectar um quadro em uma seqüência de quadros e, portanto, se constitui em um contador, o qual, quando incrementado, possibilita a estruturação de um multiquadro com 256 quadros.

\subsubsection{Descrição do overhead introduzido pela camada OPU, ODU e OTU}

Nesta seção apresentaremos a descrição das subcamadas OPU, ODU e OTU. Detalhes do overhead inserido pelas respectivas subcamadas são apresentados no Anexo IV.

O quadro da subcamada OPU é formado pelo sinal transportado e duas colunas de overhead. Em outras palavras, observando-se a Figura 5.9 (a) temos o quadro da subcamada OPU 
delimitado pelas colunas 15 a 3824. Desta maneira o overhead inserido pela subcamada OPU fornece informações para o mapeamento dos sinais clientes (por exemplo, IP, ATM) para a camada ODU, a fim de recuperá-lo apropriadamente no seu ponto final. O mapeamento de sinais clientes como, por exemplo, SONET-SDH, ATM e Gigabit Ethernet para a subcamada OPU pode ser encontrado em [62]. As taxas de transmissão da subcamada OPU são: 2,5, 10, e 40 Gbps.

O quadro da subcamada ODU, apresentado na Figura 5.9 (b), consiste do quadro OPU e do overhead da subcamada ODU. O overhead inserido por esta subcamada contém informações que habilitam a manutenção e a operação dos canais ópticos incluindo:

1. Sinais de Manutenção (MS-maintenance signals)

O overhead da subcamada ODU contêm bytes reservados para a informação de sinais de manutenção incluindo: indicação de conexão aberta (OCI- open connection indication);

2. Canais de Comunicação GCC1 e GCC2 (GCC-general communications channels) O overhead da subcamada ODU contém bytes reservados para dois canais de transmissão de informação entre elementos de rede. Estes canais podem ser utilizados para a transmissão de informações de gerenciamento entre os elementos de uma rede óptica;

3. Monitoramento de caminho (PM-path monitoring)

A função de monitoramento de caminho inclui o campo TTI (trail trace identifier), o qual permite ao operador da rede verificar o estado da conexão do canal óptico entre seus pontos inicial e final, e outros campos que permitem o monitoramento da taxa de erro de bits (BER - bit error ratio) e de defeitos na conexão;

4. Monitoramento conjunto de conexão (TCM-tandem connection monitoring) Como mencionado acima, o campo PM permite o monitoramento de uma conexão óptica entre os pontos inicial e final desta. Se o referido canal óptico atravessar diferentes redes ópticas, os campos TCM numerados de 1 a 6 habilitam aos diferentes operadores de rede o monitoramento do canal óptico dentro de seus domínios. Como 
exemplo, a Figura 5.10 mostra uma conexão óptica entre as cidades de São Francisco e Nova York. Esta conexão é monitorada nos pontos extremos pelo campo PM. Ao

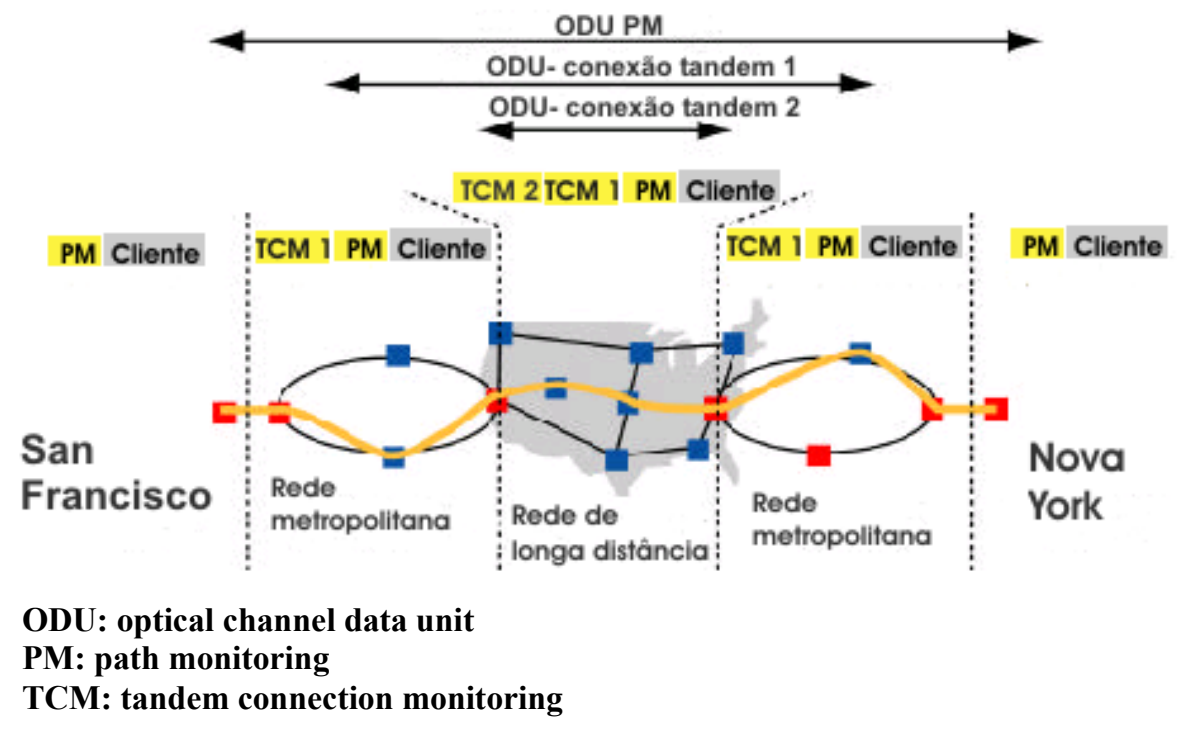

FIGURA 5.10: Monitoramento do canal óptico através de múltiplas redes utilizando-se ODU-TCM [65].

atravessar diferentes redes ópticas, o monitoramento do canal óptico em cada uma delas é realizado pelos campos TCM. Assim, o monitoramento entre redes metropolitanas é realizado pelo campo TCM 1 e o monitoramento na rede de longa distância é realizado pelo campo TCM 2. Até 7 campos TCM podem ser utilizados para o monitoramento do canal óptico. Outras formas de monitoramento poderiam ser utilizadas na Figura 5.10 como, por exemplo, designar o campo TCM 1 para o monitoramento na rede metropolitana da cidade de São Francisco, designar o campo TCM 2 para o monitoramento na rede de longa distância e designar o campo TCM 3 para o monitoramento na rede metropolitana da cidade de Nova York.

O overhead da subcamada OTU, similar a subcamada de seção da estrutura SONET/SDH, permite o monitoramento de seção por meio do campo SM (section monitoring), onde encontramos os mesmos campos presentes no monitoramento de caminho como, por exemplo, o campo TTI. Está contido também neste overhead um canal de comunicação GCC 0 que pode ser usado na comunicação entre elementos da camada de seção. Além disso, uma FEC é inserida no quadro da subcamada ODU para o monitoramento e correção de erros sobre o quadro todo e não apenas sobre o sinal transportado (payload). 


\subsubsection{Descrição dos overheads introduzidos pelas camadas OCh, OMS e OTS}

Como discutido, os overheads introduzidos pelas subcamadas OPU, ODU e OTU são superpostos ao sinal. Existem, entretanto, outros overheads introduzidos pelas camadas OCh, OMS e OTS os quais não trafegaram pelos canais de dados e sim por um canal específico, isto é, por um comprimento de onda especificamente designado, denominado OSC (optical supervisory channel). O mapeamento específico para estes overheads está em estudo e será descrito em futuras recomendações. Vale mencionar que o OSC será também utilizado para a comunicação de mensagens estabelecidas pelo plano de gerenciamento.

\subsection{PLANO DE GERENCIAMENTO ASON}

A Figura 5.11 especifica o modelo organizacional do plano de gerenciamento de uma rede ASON. Os pontos principais deste modelo são: a utilização de canais de comunicação formados pelos bytes GCC presente nas subcamadas OTU e ODU (GCC0, GCC1 e GCC2 respectivamente) e a utilização do canal OSC.

Este modelo organizacional é composto de nós gerenciados ONE (optical network element) e estações gerenciadoras WS (workstations). As WS interpretam as informações de gerenciamento que são processadas nos OS (operations system). A comunicação entre os ONEs será realizada preferencialmente através do canal OSC ou pelos bytes do campo GCC, principalmente entre ONEs localizados em domínios diferentes (inter-site communications). Já para ONEs presentes em um mesmo domínio de gerenciamento, a comunicação (intra-site communications), pode ser realizada pela forma descrita acima ou por meio de uma LAN [66]. No caso de vários domínios serem gerenciados por um único centro de gerenciamento, a comunicação será executada através de uma WAN (Figura 5.11). As redes LAN e WAN são especificadas pela recomendação ITU-T G 7712 "Architecture and Specification of Data Communication Network).

Os blocos de funções mostrados na Figura 5.11 são: MCF (message communication function), MAF (management application function), MIB (management information base), D\&T (date and time), FACAPS (fault management, configuration management, account management, performance management e security management) e AF (atomic function). 


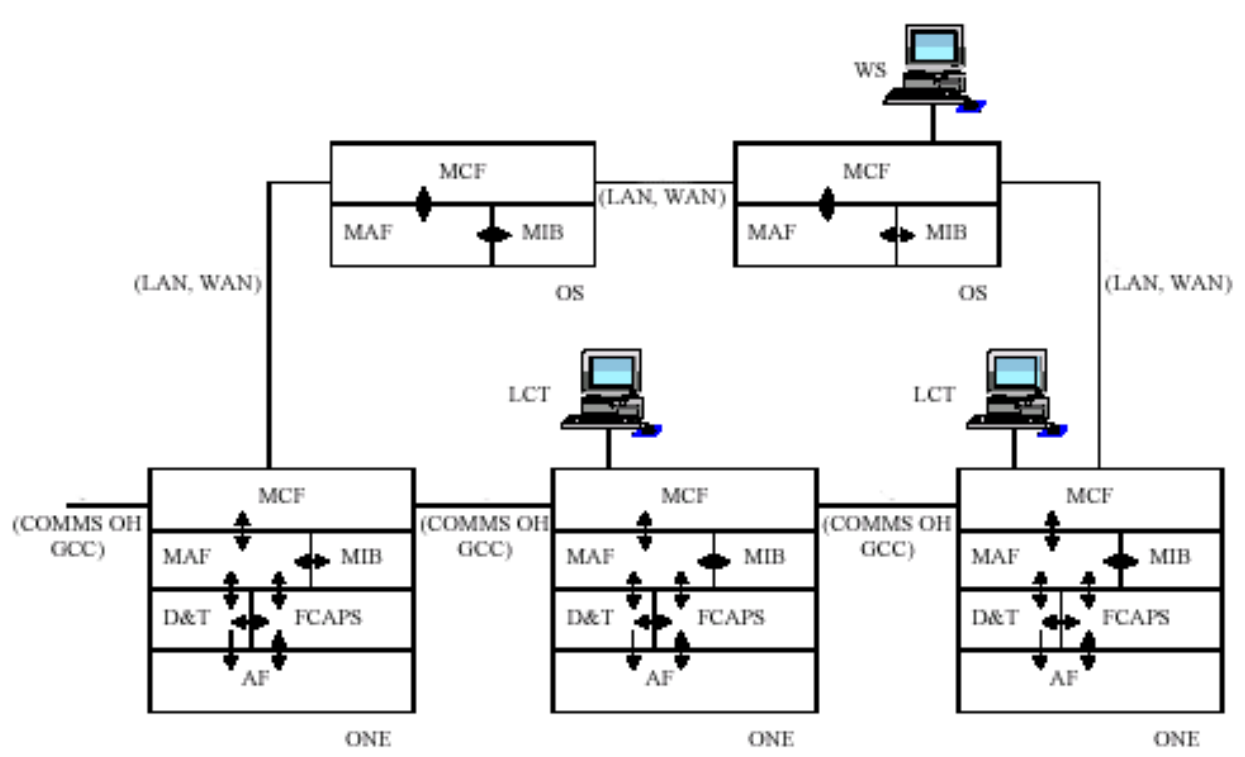

FIGURA 5.11: Modelo organizacional de gerenciamento de uma rede ASON. Terminais LCT (Local Craft Terminal) são para acesso aos objetos gerenciados.

Em linhas gerais, um sistema de gerenciamento provê mecanismos para o monitoramento, controle e coordenação de recursos. Na implementação de operações de gerenciamento os recursos são vistos como objetos gerenciáveis com propriedades definidas. Desta maneira, um sistema de gerenciamento constitui-se de funções de gerenciamento, objetos gerenciáveis e protocolos para a comunicação entre as entidades (ONES e OS, por exemplo). Atividades de gerenciamento são, assim, efetuadas por meio da manipulação de objetos gerenciáveis. $O$ conjunto de objetos gerenciáveis em um sistema, junto com seus atributos constitui o MIB. A recomendação ITU-T G 841.1 especifica as entidades gerenciadas e as propriedades de um elemento a ser gerenciado. Por exemplo, a função OCh_TTP será responsável pelo monitoramento fim a fim da camada OCh e terá a propriedade de indicar a perda do sinal (Loss of Signal). Recomendações futuras, empregando um protocolo de gerenciamento, mapearão estas propriedades em objetos gerenciáveis. A função MAF executa processos denominados de gerente e agente, sendo a origem e o término de toda e qualquer mensagem do plano de gerenciamento. Em ONEs o papel executado é o de agente, em OS o de gerente. O agente executa operações de gerenciamento com objetos gerenciáveis ou emite notificações sobre eles como conseqüência de solicitações de um gerente. Ao contrário, o gerente tem a responsabilidade de comandar o processo de gerenciamento, originar operações de gerenciamento e receber notificações. A função MCF permite aos processos gerente e agente a 
troca de mensagens de comunicação, ou seja, adapta as mensagens aos meios físicos disponíveis como, por exemplo, um canal OSC ou uma LAN.

Os propósitos de gerenciamento são categorizados em cinco áreas aglutinadas sobre as funções FACAPS. Estas funções colhem os dados provenientes do recurso gerenciado, transformando-os em objetos gerenciáveis que serão enviados para o agente. As especificações destas funções em termos de classes de objetos gerenciáveis e seus atributos são descritos na recomendação ITU-T G 841.1. As funções descritas em FACAPS são:

a) Gerenciamento de falhas

O gerenciamento de falhas é executado por um conjunto de funções que habilitam a detecção, isolação e correção de um funcionamento anormal de uma rede de transporte de dados.

b) Gerenciamento de configuração

O gerenciamento de configuração provê funções para coletar informações sobre as condições do equipamento como, por exemplo, a versão do software em uso e mudar suas configurações.

c) Gerenciamento de custo (Account management)

O gerenciamento do custo dos recursos envolvidos, será especificado em recomendações futuras.

d) Gerenciamento de performance

O gerenciamento de performance provê funções para avaliar e informar sobre o comportamento de equipamentos. Seu papel é aglutinar e analisar estatísticas de dados para o propósito de monitoramento de equipamentos.

e) Gerenciamento de segurança

O gerenciamento de segurança será especificado em recomendações futuras

As funções atômicas (AF) são definidas como as mais simples, por isto a denominação atômica, funções que permitem a obtenção dos dados para a monitoração do equipamento óptico. Todas as funções FACAPS consultam as funções atômicas. Por exemplo, quando o agente recebe uma mensagem para executar uma operação em um objeto gerenciável, aquele consulta a MIB e obtêm a informação sobre qual função FACAPS deve ser acionada e esta, por sua vez, aciona a respectiva função atômica. Por exemplo, para monitorar o canal OSC, 
mencionado anteriormente, a função atômica denominada OTSn_TT_Sk (OTS trail termination sink function) é utilizada. Esta função monitora o canal OSC e, em caso de interrupção deste canal, gera o aviso de defeito denominado dLOS-O (loss of signal overhead defect), o qual é processado pela função de gerenciamento de falhas presente no bloco FACAPS. Detalhes de outras funções atômicas são especificadas na recomendação ITU-T G 798.

A função D\&T provê informação de datas e horários, uma vez que, várias funções FACAPS necessitam deste tipo de informações para reportar o exato momento de ocorrência de uma falha. A Figura 5.12 apresenta as funções responsáveis pelo gerenciamento de um equipamento óptico.

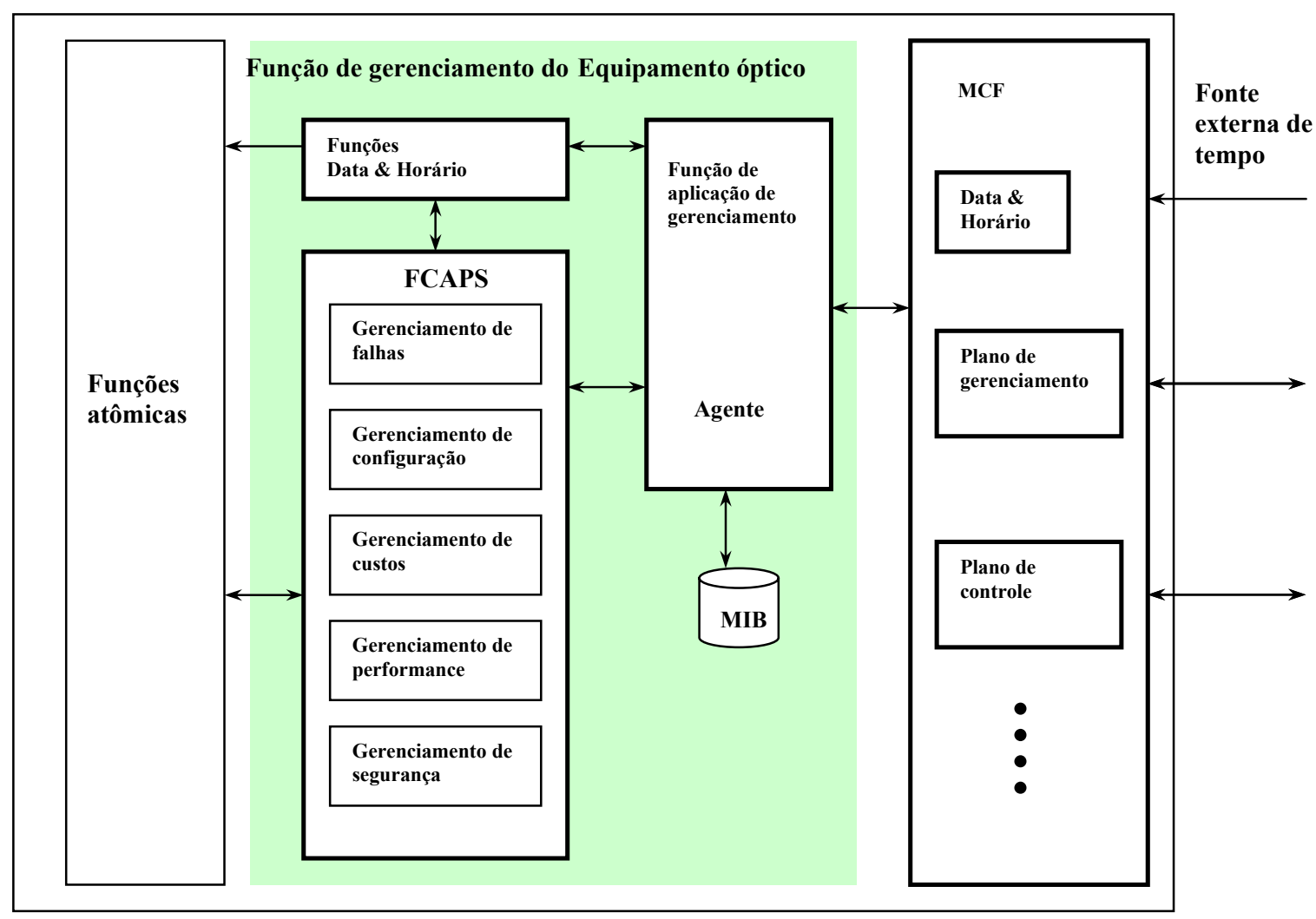

FIGURA 5.12: Funções de gerenciamento do equipamento óptico. 


\subsection{CONCLUSÕES}

O conceito de redes de transporte ópticas (OTN-optical transport network) foi criado no ITU-T a fim de acomodar diferentes clientes (IP, ATM) em redes WDM, dotá-las com a propriedade de roteamento e sinalização (networking) e gerenciá-las de uma maneira unificada. Para definir o conceito de OTN e estabelecer interoperabilidade dos equipamentos de diferentes fabricantes e entre diferentes operadores, o ITU-T desenvolveu uma série de recomendações. Tais recomendações são classificadas em termos de arquiteturas ou planos: plano de transporte, plano de gerenciamento e plano de controle.

Por serem desacopladas, interfaces de comunicação entre os referidos planos estão em estudo e serão especificadas em futuras recomendações. A interface entre o plano de controle e o plano de transporte será denominada CCI (connection controller interface) e terá o CC como a entidade gerenciadora no plano de controle. Entre o plano de controle e o plano de gerenciamento interfaces serão criadas entre os componentes CC, LRM e CallC e o plano de gerenciamento. $\mathrm{O}$ plano de gerenciamento será conectado ao de transporte por intermédio de interfaces DCN ou pelo canal OSC. Espera-se para o biênio 2003/2004 a incorporação de protocolos (por exemplo, RSVP e CR-LDP) à padronização da rede ASON. 


\section{CAPÍTULO 6}

\section{CONCLUSÕES E RECOMENDAÇÕES}

\subsection{CONCLUSÕES}

Para sobreviver no ambiente competitivo dos dias atuais, os provedores de serviços (ISPs) devem melhorar continuamente suas redes de transporte de dados e habilitar a seus usuários serviços mais rápidos e eficientes em termos de custo. No cerne da questão estão as redes ópticas, nas quais a tecnologia dominante é a fibra óptica. Este trabalho analisou a evolução de tais redes desde a sua origem no início dos anos 80, com a implantação de fibras monomodo, principalmente nos Estados Unidos, Europa e Japão, até os dias atuais onde se utilizam sistemas SONET/SDH e sistemas DWDM ponto a ponto. A introdução de equipamentos ópticos, como os amplificadores EDFAs, foi discutida, além de serem mencionadas algumas tecnologias, especificamente os MEMS (micro-electromechanical systems), para a confecção de OXCs e OADMs puramente ópticos. Estes últimos poderão dotar as redes ópticas com a função de networking, ou seja, a capacidade de manipular comprimentos de onda de forma a implementar o roteamento destes comprimentos de onda. Esta possibilidade implica em uma nova forma de relacionamento das aplicações com esta camada física.

Com o intuito de explicitar tal relacionamento apresentaram-se duas pilhas de protocolos IP/ATM/SONET-SDH e IP/PPP-HDLC/ SONET-SDH, analisou-se, para cada uma delas, a forma de encapsulamento e calculou-se o overhead introduzido. Este cálculo apontou um overhead médio ponderado de $24 \%$ para a arquitetura IP/ATM/SONET-SDH e de $2 \%$ para a arquitetura IP/PPP-HDLC/ SONET-SDH. Ressalta-se que o overhead inserido pela infraestrutura de transporte SONET/SDH (aproximadamente 4,6 \%) não está computado nos cálculos acima. De fato, estruturas multicamadas apresentam efeitos nos quais uma camada pode limitar a escalabilidade de redes inteiras, tanto quanto aumentar os custos das mesmas. Sendo assim, objetivando-se diminuir o número de camadas como forma de eliminar as limitações de desempenho devido à inserção de overheads, discutiu-se um cenário no qual a função de 
engenharia de tráfego propiciada pela camada ATM deverá ser absorvida pela camada IP e a capacidade de transporte do protocolo SONET-SDH deverá ser absorvida pela camada óptica, culminando em uma arquitetura com duas camadas, chamada IP sobre DWDM.

A primeira parte deste objetivo pode ser alcançada através da inclusão na camada IP de novas funcionalidades proporcionadas pela tecnologia MPLS (Multiprotocol Label SwitchingInternet Engineering Task Force-IETF), sendo este um plano de controle que pode ser usado não somente com roteadores, mas também em equipamentos SONET/SDH. Com este intuito foram analisados os conceitos básicos da arquitetura MPLS e demonstrou-se como tais conceitos podem ser empregados visando dotar redes IP com engenharia de tráfego. A funcionalidade restante pode ser alcançada na medida em que comutadores ópticos forem empregados em conjunto com os sistemas DWDM, transformando, ou melhor, dotando, esta camada de meios para realizar a comutação óptica.

A arquitetura com duas camadas surge assim com a utilização de Generalized MPLS (GMPLS) e a disponibilização de sistemas DWDM com comutação óptica. A arquitetura GMPLS visa prover às redes ópticas um único plano de controle, derivado do MPLS, ou seja, algumas modificações e adições são necessárias para que os protocolos de sinalização e roteamento presentes no MPLS se adaptem às peculiaridades da comutação óptica. Apresentaram-se, assim, as extensões aplicadas aos protocolos de roteamento (OSPF e IS-IS), aos protocolos de sinalização (RSVP e CR-LDP) e introduziu-se um novo protocolo, o LMP, visando este gerenciar enlaces ópticos. Com esta tríade de conceitos, o GMPLS é estendido para incluir um grupo de elementos de redes que não tomam decisões de comutação e roteamento baseados somente nas informações carregadas nos cabeçalhos de pacotes ou células, mas sim baseados em intervalos de tempo (time slots), comprimentos de onda, ou portas. A arquitetura MPLS original está, assim, sendo estendida para incluir dispositivos cujos planos de envio não reconhecem pacotes e não podem enviar dados baseados em informações neles contida. Especificamente, tais equipamentos incluem dispositivos nos quais a decisão de envio é baseada em intervalos de tempo (time slots), comprimentos de onda (wavelengths) ou portas físicas (OXCs).

Em relação aos aspectos de padronização das redes ópticas foram mencionadas várias instituições de padronização, OIF, IETF e ITU-T. Enquanto o OIF e o IETF trabalham na padronização de um plano de controle para as redes ópticas, o ITU-T padroniza, por meio dos grupos de estudos 13 e 15, não somente o plano de controle como também a arquitetura inteira de uma rede óptica. Tal arquitetura foi avaliada em relação às recomendações que especificam 
os planos de controle, gerenciamento e transporte que a compõem. A estrutura do plano de controle foi apresentada, bem como a abordagem relacionada à inserção da arquitetura GMPLS à arquitetura de redes ópticas padronizada pelo ITU-T (ASON). Esta inserção justifica-se, uma vez que as redes ópticas padronizadas pelo ITU-T utilizam um plano de controle, o qual pode ser composto pelos protocolos empregados pelo plano de controle GMPLS.

\subsection{RECOMENDAÇÕES PARA TRABALHOS FUTUROS}

Um aspecto importante no prosseguimento desta pesquisa será estudar, embasado nos conceitos apresentados nesta dissertação, os passos necessários para a implantação de tais conceitos nas redes de transmissão de dados já em funcionamento. Ou seja, como a implantação das arquiteturas padronizadas pelos institutos IETF, OIF e ITU-T pode ser implementada nas redes atuais, sem causar paralisações da prestação de serviços e prejuízos para os provedores de serviços (ISP). 


\section{REFERÊNCIAS BIBLIOGRÁFICAS}

[1] RYAN J. P.; KENT R. H., "WDM: North American Deployment Trends". IEEE Communications Magazine, v.36, n.2, p.40-44, Fevereiro 1998.

[2] TANENBAUM A. S., "Redes de Computadores".3ª ed. São Paulo, Editora Campus, p.144-147,1997.

[3] ALFERNESS R. C.; BONENFANT P. A.; NEWTON C. J.; SPARKS K.A.; VARMA E. L. A., "Pratical Vision for Optical Transport Networking". Bell Labs Technical Journal, v.4, n.1, p.3-18, Janeiro - Março 1999.

[4] AGRAWAL G.P., "Fiber-optic Comunication System"-2 2 ed, EUA, Wiley-Interscience, Cap 3, p.75-128,1997.

[5] Fundamentals of DWDM Technology, Cisco Technology Papers, http://www.cisco.com/univercd/cc/td/doc/product/mels/dwdm/dwdm_ovr.html Dezembro 2000.

[6] W. SULHOFF J.; SRIVASTAVA A. K.; SUN Y.; ZHOU J., "Optical Fiber Amplifiers for WDM Optical Networks". Bell Labs Technical Journal, v.4, n.1. p.187-206, Janeiro-Março 1999.

[7] GILES C. R.; SPECTOR M.,'The Wavelength Add/Drop Multiplexer for Lighwave Communication Networks". Bell Labs Technical Journal, v.4, n.1, p.207-229, Janeiro Março 1999. 
[8] Optical Networks, Web Proforum Tutorials- Alcatel. The Internacional Engineering Consortium, http://www.iec.org, Dezembro 1998.

[9] BRANCALION JOSÉ FERNANDO BASSO, "Avaliação das limitações de desempenho em multiplexadores "add/drop" para redes fotônicas WDM”. São Carlos Dissertação (Mestrado) - Escola de Engenharia de São Carlos, Universidade de São Paulo, 2001.

[10] CHU P.B.; LEE S.S.;PARK S., MEMS: "The path to large Optical Crossconnects". IEEE Communications Magazine, v.40, n.3, p.80-87, Março 2002.

[11] TZE-WEI Y.; EDDIE K.L.;GOLDENBERG A., "Mems Optical Switches". IEEE Communications Magazine, v.39, n11, p.158-163, Novembro 2001.

[12] LIN Y.L.; GOLDSTEIN L.E.; TKACH R.W., "Free-space Micromachined Optical Switches for Optical Networking”. IEEE J. Sel. Topics Quantum Elect., vol. 5, n 1, p.4-9, Janeiro 1999.

[13] REFI J. J., "Optical Fibers for Optical Networking”. Bell Labs Technical Journal, v.4, n.1, p.246-261, Janeiro- Março 1999.

[14] ROSEN E.; VISWANATHAN A.; CALLON R., "Multiprotocolol Label Switching Arquiteture". Internet Engineering Task Force. IETF RFC 3031, Janeiro 2001. http://www.ietf.org.

[15] GREEN P., "Progress in Optical Networking". IEEE Communications Magazine, v.39, n.1, p.54-61, Janeiro 2001.

[16] LAUBACH M., "Classical IP and ARP over ATM". Internet Engineering Task Force, IETF RFC 1577, Janeiro 1994, http://www.ietf.org. 
[17] MALIS A.; SIMPSON W., "PPP over SONET/SDH”. Internet Engineering Task Force, IETF RFC 2615, Junho 1999, http://www.ietf.org.

[18] SIMPSON W., "The Point-to-Point Protocol (PPP)". Internet Engineering Task Force, IETF RFC 1661, Julho 1994, http://www.ietf.org.

[19] THOMSON K.; MILlER J.G.; WILDER R., "Wide Area Traffic Patterns and Characteristics", IEEE Network, vol.11, n 6, p. 10-23, Novembro-Dezembro. 1997. http://www.vbns.net/presentations/papers/MCI/traffic.ps.gz

[20] ANDERSON J.; MANCHESTER J.; RODRIGUEZ-MORAL A.; VEERARAGHAVAN M., "Protocols and Architectures for IP Optical Networking". Bell Labs Technical Journal, v.4, n.1, p.105-124, Janeiro - Março 1999.

[21] BONENFANT P.; MORAL R. A., "Framing Techiques for IP over Fiber", IEEE Network, vol.15, n 4, p. 12-18, Julho - Agosto 2001.

[22] VISWANATHAN A.;FELDMAN N.;WANG Z.;CALLON R., "Evolution of Multiprotocol Label Switching”. IEEE Communications Magazine, v.36, n.5, p.165172, Maio 1998 .

[23] REKHTER Y.;ROSEN E., "Carrying Label Information in BGP-4". Internet Engineering Task Force, IETF RFC 3107, Maio 2001. http://www.ietf.org.

[24] AWDUCHE D., BERGER L.; GAN D.; Li T.; SRINIVASAN V.; SWALLOW G., "RSVP-TE: Extensions to RSVP for LSP Tunnels". Internet Engineering Task Force, IETF RFC 3209, Dezembro 2001. http://www.ietf.org. 
[25] ANDERSSON L.; DOOLAN P.; FELDMAN N.; FREDETTE A..; THOMAS B., "LDP Specification". Internet Engineering Task Force, IETF RFC 3036, Janeiro 2001. http://www.ietf.org.

[26] JAMOUSSI B.; ANDERSSON L.; CALLON R.; DANTU R.; WU L.; DOOLAN P.; WORSTER T.; FELDMAN N.; FREDETTE A.; GIRISH M.; GRAY E.; HEINANEN J.; KILTY J.; MALIS A., "Constraint-Based LSP". Internet Engineering Task Force, IETF RFC 3212, Janeiro 2002. http://www.ietf.org.

[27] ROSEN E.; TAPPAN D.; FEDORKOW G.; REKHTER Y.; FARINACCI D.; LI T.; CONTA A.,"MPLS Label Stack Encoding”. Internet Engineering Task Force, IETF RFC 3032, Janeiro 2001. http://www.ietf.org.

[28] LAWRENCE J., "Designing Multiprotocol Label Switching Networks". IEEE Communications Magazine, v.39, n.7, p.134-142, Julho 2001.

[29] THOMAS B.; GRAY E., "LDP Applicability". Internet Engineering Task Force, IETF RFC 3037, Janeiro 2001. http://www.ietf.org.

[30] ASH J.; GIRISH M.; GRAY E.; JAMOUSSI B.; WRIGHT G., "Applicability Statement for CR-LDP” . Internet Engineering Task Force, IETF RFC 3213, Janeiro 2002. http://www.ietf.org.

[31] AWDUCHE D.; HANNAN A.; XIAO X., "Applicability Statement for Extensions to RSVP for LSP-Tunnels". Internet Engineering Task Force, IETF RFC 3210, Dezembro 2001. http://www.ietf.org.

[32] BRITTAIN P.; FARREL A., "MPLS Traffic Engineering: A choice of Signaling Protocols". Data Connection, Janeiro 2000, http://www.dataconnection.com. 
[33] GHANWANI A.; ASHWOOD-SMITH P.; LI L.; FELDMAN N., "Traffic Engineering Standards in IP Networks using MPLS”. IEEE Communications Magazine, v.37, n.12, p.49-53, Dezembro 1999.

[34] AWDUChE D.; MALCOLM J.; AgOGBUA J.; O'DELl M.; MCMANUS J., "Requirements for Traffic Engineering Over MPLS". Internet Engineering Task Force, IETF RFC 2702, Setembro 1999. http://www.ietf.org.

[35] KATZ D.; YEUNG D.; KOMPELLA K., "Traffic Engineering Extensions to OSPF Version 2”. Internet Engineering Task Force, Internet draft, Agosto 2002. Work in progress. http:// draft-katz-yeung-ospf-traffic-07.txt.

[36] SMIT H., LI T., "ISIS Extensions for Traffic Engineering”. Internet Engineering Task Force, Internet draft, Dezembro 2002. Work in progress.

http:// draft-ietf-isis-traffic-04.txt

[37] BOYLE J.; GILL V.; HANNAN A.; COOPER D.; AWDUCHE D.; CHRISTIAN B.; Lai W., "Applicability Statement for Traffic Engineering with MPLS” . Internet Engineering Task Force, IETF RFC 3346, Agosto 2002. http://www.ietf.org.

[38] MANNIE E.; ASHWOOD-SMITH P.; AWDUCHE D.; BANERJEE A.; BASAK D.; BERGER L. et al., "Generalized Multi-Protocol Label Switching (GMPLS) Architecture" . Internet Engineering Task Force, Internet draft, Março 2002. Work in progress. http:// draft-ietf-ccamp-gmpls-architecture-02.txt.

[39] BERGER L.; ASHWOOD-SMITH P.; BANERJEE A.; BERNSTEIN G.; DRAKE J. et al., "Generalized MPLS - Signaling Functional Description". Internet Engineering Task Force, Internet draft, Abril 2002. Work in progress. http:// draft-ietf-mpls-generalized-signaling-08.txt. 
[40] BERGER L.; ASHWOOD-SMITH P.; BANERJEE A.; BERNSTEIN G.; DRAKE J et al. "Generalized MPLS Signaling - RSVP-TE Extensions". Internet Engineering Task Force, Internet draft, Abril 2002. Work in progress.

http:// draft-ietf-mpls-generalized-rsvp-te-07.txt.

[41] ASHWOOD-SMITH P.; BERGER L.; BANERJEE A.; BERNSTEIN G.; DRAKE J. et al., "Generalized MPLS Signaling - CR-LDP Extensions". Internet Engineering Task Force, Internet draft, Abril 2002. Work in progress. http:// draft-ietf-mpls-generalized-cr-ldp-06.txt.

[42] MANNIE E.; PAPADIMITRIOU D.; ANSORGE S.; ASHWOOD-SMITH P.; BANERJEE A. et al., "Generalized Multiprotocol Label Switching Extensions for SONET and SDH Control". Internet Engineering Task Force, Internet draft, Junho 2002. Work in progress . http:// draft-ietf-ccamp-gmpls-sonet-sdh-05.txt.

[43] PAPADIMITRIOU D.; BELLATO A.; DHARANIKOTA S.; FONTANA M. at al. "Generalized MPLS Signalling Extensions for G.709 Optical Transport Networks Control”. Internet Engineering Task Force, Internet draft, Junho 2002. Work in progress. http:// draft-ietf-ccamp-gmpls-g709-01.txt.

[44] BANERJEE A.; DRAKE J.; LANG J.; TURNER B.; AWDUCHE D.; BERGER L.; KOMPELLA K.; REKHTER Y., "Generalized Multipotocol Label Switching: An Overview of Sinaling Enhancements and Recovery Tecniques". IEEE Communications Magazine, v.39, n.7, p.144-151, Julho 2001.

[45] KOMPELLA K.; REKHTER Y.; BANERJEE A.; DRAKE J.; BERNSTEIN G.; FEDYK D.; MANNIE E.; SAHA D.; SHARMA V., "OSPF Extensions in Support of Generalized MPLS”. Internet Engineering Task Force, Internet draft, Maio 2002. Work in progress. http:// draft-ietf-ccamp-ospf-gmpls-extensions-07.txt 
[46] KOMPELLA K.; REKHTER Y.; BANERJEE A.; DRAKE J.; BERNSTEIN G.; FEDYK D.; MANNIE E.; SAHA D.; SHARMA V., "IS-IS Extensions in Support of Generalized MPLS”. Internet Engineering Task Force, Internet draft, Junho 2002. Work in progress. http:// draft-ietf-isis-gmpls-extensions-13.txt.

[47] KOMPELLA K.; REKHTER Y., "Signalling Unnumbered Links in RSVP-TE". Internet Engineering Task Force, Internet draft, Julho 2002. Work in progress. http:// draft-ietf-mpls-rsvp-unnum-07.txt.

[48] KOMPELLA K.; REKHTER Y.; KULLBERG A., "Signalling Unnumbered Links in CR-LDP”. Internet Engineering Task Force, Internet draft, Julho 2002. Work in progress. http:// draft-ietf-mpls-crldp-unnum-07.txt.

[49] KOMPELLA K.; REKHTER Y.; BANERJEE A.; DRAKE J.; BERNSTEIN G.; FEDYK D.; MANNIE E.; SAHA D.; SHARMA V.; BASAK D.; BERGER L., "Routing Extensions in Support of Generalized MPLS” . Internet Engineering Task Force, Internet draft, Abril 2002. Work in progress.

http:// draft-ietf-ccamp-gmpls-routing-04.txt.

[50] BANERJEE A.; DRAKE J.; LANG J.; TURNER B.; KOMPELLA K.; REKHTER Y., "Generalized Multipotocol Label Switching: An Overview of Routing and Management Enhancements”. IEEE Communications Magazine, v.39, n.1, p.144-150, Janeiro 2001.

[51] KOMPELLA K.; REKHTER Y., "LSP Hierarchy with Generalized MPLS TE" . Internet Engineering Task Force, Internet draft, Junho 2002. Work in progress. http:// draft-ietf-mpls-lsp-hierarchy-07.txt.

[52] KOMPELLA K.; REKHTER Y.; BERGER L., "Link Bundling in MPLS Traffic Engineering". Internet Engineering Task Force, Internet draft, Julho 2002. Work in progress. http:// draft-ietf-mpls-bundle-04.txt. 
[53] FARREL A., "MPLS in Optical Network. Data Connection", Outubro 2001, http://www.dataconnection.com.

[54] BERNSTEIN G.; RAJAGOPALAN B.; PENDARAKIS D.; CHIU A.; HUJBER F.; STRAND |J.; SHARMA V.; DHARANIKOTA S.; CHENG D.; IZMAILOV R., "Optical Inter Domain Routing Considerations". Internet Engineering Task Force, Internet draft, Fevereiro 2002. Work in progress .

http:// draft-ietf-ipo-optical-inter-domain-01.txt.

[55] BONENFANT P.; MCGUIRE A., "Standards: The Blueprints for Optical Networking". IEEE Communications Magazine, v.36, n.2, p.68-78, Fevereiro 1998.

[56] ITU-T REC. G 8080, "Architecture for the Automatically Switched Optical Networks (ASON)", Novembro 2001.

[57] HALEVI C.; WANG B., "Standards Issues Analysis Report on the ITU-T Study Group 15 Meeting of April 29 - May 10, 2002". Telcordia Standards Knowledgebase. http://tsk.telcordia.com, Maio 2002.

[58] LARKIN N., "ASON and GMPLS-The Battle of the Optical Control Plane". http://www.dataconnection.com, Agosto 2002.

[59] PAPADIMITRIOU D.; LIN Z.; PENDARAKIS D., "Generalized MPLS (GMPLS) RSVP-TE Usage and Extension For Automatically Switched Optical Network (ASON)". Internet Engineering Task Force, Internet draft, Agosto 2002. Work in progress. http:// draft-lin-ccamp-gmpls-ason-rsvpte-01.txt.

[60] ABOUL-MAGD O., "CR-LDP Extensions for ASON". Internet Engineering Task Force, Internet draft, Junho 2002. Work in progress . http:// draft-aboulmagd-ccamp-crldp-ason-ext-00.txt. 
[61] ABOUL-MAGD O.; JAMOUSSI B.; SHEW S.; GRAMMEL G.; BELOTTI S.; PAPADIMITRIOU D., "Automatic Switched Optical Network (ASON) Architecture and Its Related Protocols ". Internet Engineering Task Force, Internet draft, Março 2002. Work in progress . http:// draft-ietf-ipo-ason-02.txt .

[62] ITU-T REC. G 709 “Interface for the optical transport network (OTN)", Fevereiro 2001.

[63] ITU-T REC. G 959.1 "Optical transport network physical layer interfaces", Fevereiro 2001.

[64] ITU-T REC. G 872 "Architecture of optical transport networks", Novembro 2001.

[65] THELANDER HEIDI, "Digital Wrapper Standards Tutorial”, Julho 2001.

[66] ITU-T REC. G 841 "Management aspects of the optical transport network element", Novembro 2001. 


\section{APÊNDICE I}

\section{I.1 SIGNIFICADO DOS BLOCOS DE UM QUADRO AAL-5}

Um quadro AAL-5 (Figura I.1), utilizado no encapsulamento IP/ATM é composto de blocos com os respectivos significados descritos abaixo:

AAL5 - Frame

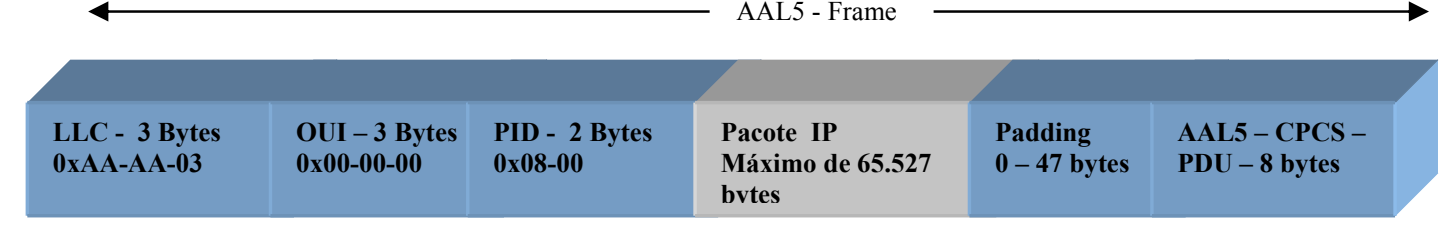

FIGURA I.1: Quadro AAL-5, utilizado no encapsulamento IP/ATM.

1. Cabeçalho SNAP: O campo LLC codificado para OXAA-AA-03 indica presença de encapsulamento LLC com cabeçalho SNAP. O campo OUI (organizationally unique identifier de 3 bytes) administra o significado dos dois bytes seguintes do PID (protocol identifier). Juntos eles identificam um protocolo de roteamento diferenciado. O valor OUI - 0x00-00-00 especifica que o PID a seguir é de um protocolo de rede. No caso particular do protocolo IP o valor deste será 0x08-00;

2. O campo Padding (enchimento) é usado para encaixar o quadro exatamente dentro de uma célula ATM, ou seja, para que a última célula ATM leve o campo CPSC-PDU de oito bytes, integralmente. $\mathrm{O}$ campo de payload conterá informações de usuário até um máximo de $2^{16-1}$ bytes;

3. O bloco AAL-5 CPCS-PDU (common part convergence sublayer-protocol data unit), descrito na Figura I.2, é composto de oito bytes com os respectivos significados descritos abaixo: 


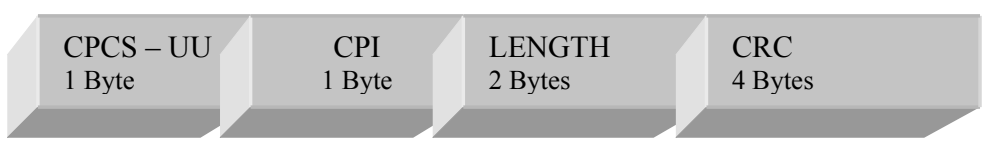

FIGURA I.2: Significado dos 8 bytes do Frame (AAL5-CPCS-PDU)

a) CPSC-UU (user to user indication): Este campo é usado para transferir informação CPSC - User to User, não apresentando função para o encapsulamento multiprotocolo ATM (RFC 1493), podendo ser codificado com qualquer valor;

b) CPI (common part indicator): Designa o CPCS -PDU para 64 bits ( 8 bytes) sendo codificado para o valor $0 \mathrm{X} 00$;

c) LENGTH: Indica o comprimento, em bytes, do campo de payload. O máximo valor para este campo é 65.535 bytes;

d) CRC (cyclic redundancy check): É o campo onde se coloca o resultado do processo de verificação de erro, aplicado à mensagem inteira, incluindo o enchimento e o fecho desta;

\section{I.2 SIGNIFICADO DOS BLOCOS DE UM QUADRO PPP-HDLC}

O processo de mapeamento PPP/HDLC, cujo quadro esta descrito na Figura I.3, começa e termina pelo byte de flag padrão (01111110- Hexadecimal 0x7E), utilizado para sincronização, o qual é complementado (byte stuffing) com caracteres, quando sua presença é detectada dentro de um campo de carga útil de dados do usuário. Esta complementação é feita no transmissor da seguinte maneira: quando detectada a seqüência $0 \times 7 \mathrm{E}$, esta é mudada para a seqüência $0 \times 7 \mathrm{D}$ 0x5E. Igualmente, quando detectada a seqüência de escape 0x7D esta é mudada para 0x7D 0x5D. Na recepção, os padrões inseridos são removidos e substituídos pelas seqüências originais. Em seguida, temos o campo endereço, sendo definido para o valor binário 11111111 (Hexadecimal-0xFF), indicando que todas as estações devem aceitar o quadro. A utilização desse valor evita o problema decorrente da necessidade de atribuição de endereços de enlace de dados. 
O campo controle é exibido na seqüência e seu valor padrão é 00000011 [67]. Esse valor indica um quadro não-numerado, ou seja, o PPP não oferece uma transmissão confiável através da utilização de números de seqüência e confirmações como o padrão. Em outras palavras, os quadros PPP não são numerados, não existindo por parte do transmissor e do receptor a necessidade de se aguardar por uma confirmação de recebimento destes. O quarto campo é o campo protocolo, cuja tarefa é informar o tipo de pacote contido no campo de informação. Os códigos são definidos para LCP (Link Control Protocol), IP, IPX (Internet Protocol Exchange) e outros protocolos. Detalhes podem ser encontrados em [18].

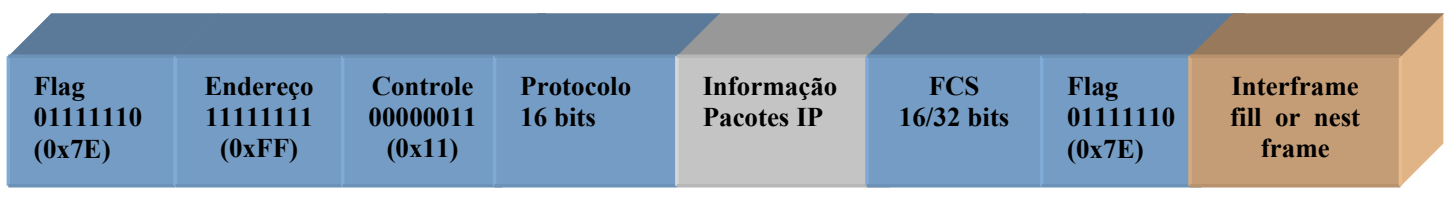

FIGURA I.3: Quadro PPP-HDLC, utilizado no encapsulamento IP/SONET-SDH.

O campo de informação tem comprimento variável, podendo se estender até o tamanho máximo negociado. Se o comprimento não for negociado, será utilizado um comprimento padrão de 1500 bytes. Poderá haver um enchimento (padding) depois do campo informação, se necessário.

O campo frame check sequence (FCS) com 16 bits utiliza o polinômio " $1+\mathrm{x}^{5}+\mathrm{x}^{12}+\mathrm{x}^{16}$ " para verificar erros nos demais campos (flag, endereço, controle, protocolo e informação). Entretanto o campo FCS com 32 bits é mais utilizado, empregando o polinômio " $1+\mathrm{x}^{1}+\mathrm{x}^{2}+$ $\mathrm{x}^{4}+\mathrm{x}^{5}+\mathrm{x}^{7}+\mathrm{x}^{8}+\mathrm{x}^{10}+\mathrm{x}^{11}+\mathrm{x}^{12}+\mathrm{x}^{16}+\mathrm{x}^{22}+\mathrm{x}^{23}+\mathrm{x}^{26}+\mathrm{x}^{32}$, Este mapeamento executa $\mathrm{o}$ processo de embaralhamento (scrambler) antes de inserir os dados em um enlace SONET/SDH. MANCHESTER et al [68] fornecem mais detalhes sobre este mapeamento.

Durante períodos ociosos quando não há dados a serem transmitidos, o flag padrão (01111110- Hexadecimal 0x7E), é transmitido como um byte de preenchimento (interframe fill), com o intuito de manter a sincronização. Além disto, o fato do interframe fill ser o delineador do começo e fim de um quadro, ao recebê-lo, um receptor estará sempre em um estado de alerta esperando o começo de um quadro.

A Figura I.4 mostra a disposição de blocos de funções executadas no mapeamento de IP/PPP/HDLC/SONET/SDH. 
TRANSMISSÃO

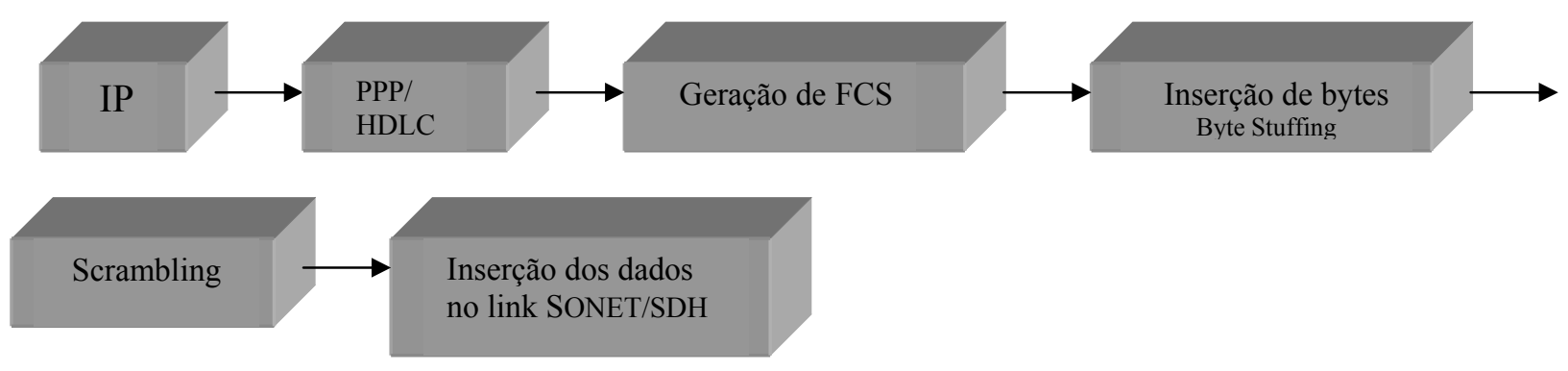

\section{RECEPÇ̃̃O}

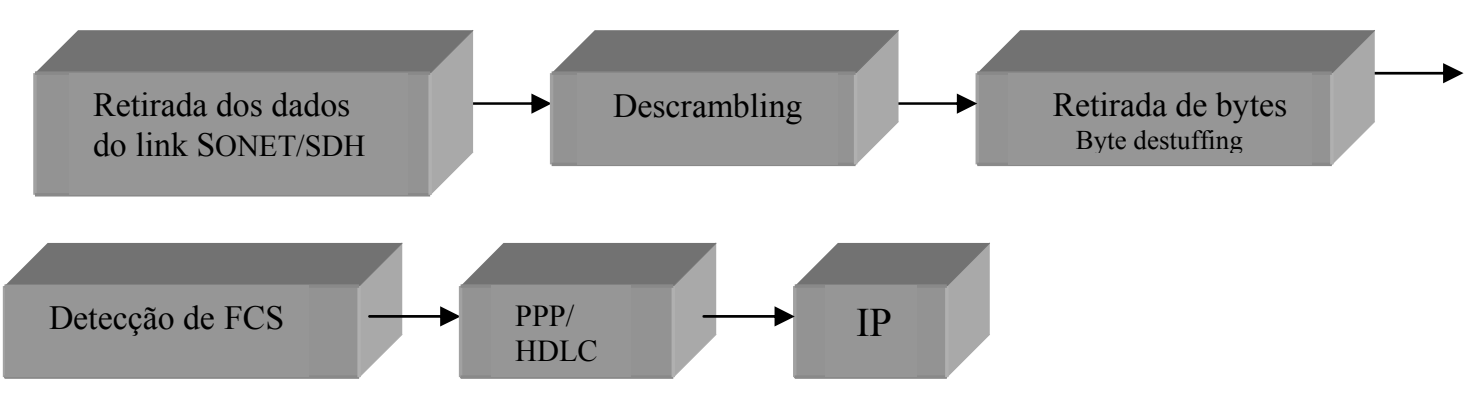

FIGURA I.4: Bloco de funções executados no mapeamento IP/PPP/HDLC/SONET/SDH.

\section{I.3 DETALHAMENTO DOS CÁLCULOS DE OVERHEAD}

\section{I.3.1 Overhead inserido pela encapsulação IP/ATM}

a-) Encapsulação ATM para pacotes IP com tamanho de 40 bytes

Overhead inserido pelo frame AAL-5 para cada pacote de informação.

$$
3(\mathrm{LLC})+3(\mathrm{OUI})+2(\mathrm{PID})+8(\mathrm{AAL}-5 \text { CPCS- PDU })=16 \text { bytes }
$$

Pacote IP 40 bytes +16 bytes overhead $=56$ bytes

1 célula de 48 bytes 
1 célula de 8 bytes +40 bytes (enchimento)

Overhead total $=16+40+10(2$ células ATM com cinco bytes de overhead cada $)=66$ bytes

$\%$ Overhead $=66 / 40=165,0 \%$

b-) Encapsulação ATM para pacotes IP com tamanho de 1500 bytes

Pacote IP 1500 bytes +16 bytes overhead $=1516$ bytes

31 células de 48 bytes

1 célula de 28 bytes +20 (enchimento)

Overhead total $=16($ Eq. 2.1$)+20+10(32$ células ATM com cinco bytes de overhead cada $)=$ 196 bytes

$\%$ Overhead $=196 / 1500=13,1 \%$

c-) Encapsulação ATM para pacotes IP com tamanho de 552 bytes

Pacote IP 552 bytes +16 bytes overhead $=568$ bytes

11 células de 48 bytes

1 célula de 40 bytes +8 bytes (enchimento)

Overhead total $=16$ (Eq. 2.1) $+8+60(12$ células ATM com cinco bytes de overhead cada $)=84$ bytes

$\%$ Overhead $=84 / 552=15,2 \%$

d-) Encapsulação ATM para pacotes IP com tamanho de 44 bytes

Pacote IP 44 bytes +16 bytes overhead $=60$ bytes

1 célula de 48 bytes

1 célula de 12 bytes +36 bytes (enchimento) 
Overhead total $=16$ (Eq. 2.1) $+36+10(2$ células ATM com cinco bytes de overhead cada $)=62$ bytes

$\%$ Overhead $=62 / 44=140,9 \%$

e-) Encapsulação ATM para pacotes IP com tamanho de 576 bytes

Pacote IP 576 bytes +16 bytes overhead $=592$ bytes

12 células de 48 bytes

1 célula de 16 bytes +32 bytes (enchimento)

Overhead total $=16($ Eq. 2.1$)+32+65(13$ células ATM com cinco bytes de overhead cada $)=$ 113 bytes

$\%$ Overhead $=113 / 576=19,6 \%$

\section{I.3.1.1 Cálculo do overhead médio ponderado para a arquitetura IP/ATM}

Neste item calcularemos o overhead médio ponderado da arquitetura IP/ATM para a distribuição de tamanhos de pacotes descrita na Tabela 2.1.

Sendo $X$ a média ponderada dos bytes de overhead sobre a distribuição do tamanho dos pacotes e $Y$ a média ponderada do tamanho dos pacotes sobre a distribuição do tamanho dos pacotes, então

Overhead médio ponderado $=\frac{X}{Y}$

$X=\frac{(66 \times 0,389+196 \times 0,115+84 \times 0,101+62 \times 0,061+113 \times 0,049)}{0,389+0,115+0,101+0,061+0,049}$

$X=92,3$ bytes de overhead médio ponderado 


$$
Y=\frac{(40 \times 0,389+1500 \times 0,115+552 \times 0,101+44 \times 0,061+576 \times 0,049)}{0,389+0,115+0,101+0,061+0,049}
$$

$Y=384,2$ bytes do pacote IP médio ponderado

e $\quad \frac{X}{Y}=\frac{92,3}{384,2} \times 100=24,0 \%$

\section{I.3.2 Overhead inserido pelo encapsulação IP/PPP/HDLC}

A porcentagem de overhead associado com o mapeamento IP/PPP/HDLC é o resultado dos 8 bytes fixos de overhead inseridos, independentemente do tamanho do pacote de informação IP a ser transportado. Ressalta-se assim a utilização do campo FCS com o tamanho default de 16 bits.

\section{I.3.2.1 Cálculo do overhead médio ponderado para a arquitetura IP/PPP/HDLC}

Neste item calcularemos o overhead médio da arquitetura IP/PPP/HDLC para a distribuição de tamanhos de pacotes descrita na Tabela 2.1.

Sendo $Z$ igual aos oitos bytes fixos de overhead inseridos independente do pacote IP a ser transportado.

Overhead médio $=\frac{Z}{Y}$

Onde $\quad Z=8$ e $Y=$ Eq.(2.3)

Assim, overhead médio ponderado $=2,1 \%$ 


\section{APÊNDICE II}

\section{II.1 ROTEAMENTO IP}

O processo de roteamento na Internet tem por base o endereço IP de cada computador. Entretanto, tais endereços não podem ser usados para o envio de pacotes, pois o hardware da camada de enlace não os interpreta corretamente.

Tendência dominante nos dias atuais, a conexão entre computadores ocorre através de LANs, nas quais a interligação é realizada por intermédio de placas de interface que só utilizam endereços LAN. Por exemplo, no caso de redes Ethernet, cada placa de rede fabricada é equipada com endereços de 48 bits solicitados pelo fabricante ao IEEE (Institute of Eletrical and Electronics Engineers), assegurando-se, assim, a impossibilidade de duas placas apresentarem o mesmo endereço. Tais placas enviam e recebem quadros com base em endereços Ethernet de 48 bits, nada sabendo sobre endereços IP de 32 bits. Cada rede IP é designada por um prefixo de destinação.

Uma rede ou prefixo de destinação é um grupo de endereços IP que podem ser tratados similarmente para propósitos de endereçamento. Por exemplo, o prefixo de endereçamento 10.1.1.0/24 representa a faixa de endereços IP no qual os primeiros três octetos (24 bits) são respectivamente 0000 1010, 0000 0001, 0000 0001, com qualquer valor para o último octeto. Em outras palavras, o prefixo de destinação 10.1.1.0/24 representa qualquer endereço 10.1.1.x onde x é um valor entre 0 e 256, inclusive. O mapeamento de endereços IP em endereços Ethernet pode ser explicado através da Figura II.1, na qual são apresentadas duas redes Ethernet interligadas por um backbone formado por três roteadores. A primeira rede Ethernet terá o endereço IP 192.31.65.0/24 e a outra o endereço 192.31.63.0/24, sendo ambas interligadas, por simplicidade, por apenas três roteadores cujo endereço de rede IP é 192.31.60.0/24.

Se o usuário do computador 1 desejar se comunicar com o computador 2, o primeiro passo seria a descoberta do endereço IP do host 2, tarefa executada pelo DNS (Domain Name System). De posse do endereço IP, o software da camada superior do computador 1 constrói um pacote com o endereço IP 192.31.65.5 no campo destination address e o fornece ao software IP, o qual 
examinará o endereço e constatará a presença deste em sua própria rede, necessitando agora seu endereço Ethernet.

Os roteadores $\mathbf{R} 1$ e $\mathbf{R 2}$

possuem 2 endereços IP

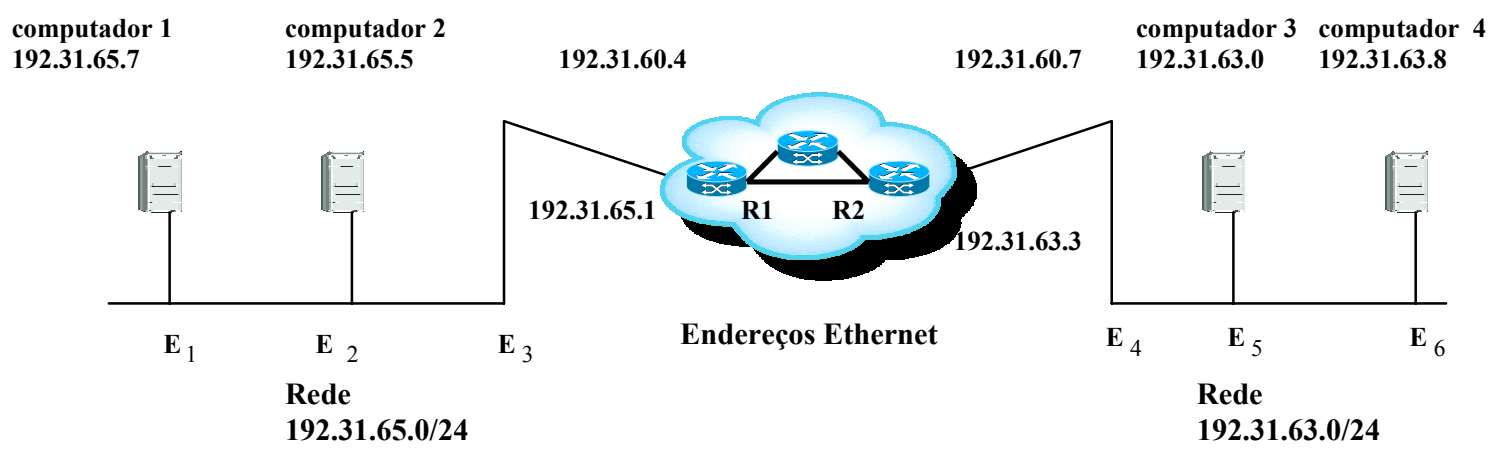

FIGURA II.1:Interconexão de duas redes Ethernet por um backbone IP.

O computador 1 envia um pacote de difusão na rede Ethernet, perguntando: "A quem pertence o endereço IP 192.31.65.5?”. A difusão chegará a todos os computadores da rede Ethernet 192.31.65.0/24 e cada um deles verificará seu próprio endereço IP. Somente o computador 2 responderá com seu endereço Ethernet (E2). Dessa forma, o computador 1 descobrirá o endereço Ethernet E2 do endereço IP 192.31.65.5. O protocolo responsável por fazer este questionamento e obter a resposta é chamado ARP (address resolution protocol - IETF RFC 826). Assim, o software IP do computador 1 constrói um quadro Ethernet endereçado a E2, coloca o pacote IP (endereçado a 192.31.65.5) no campo de carga útil e envia para a Ethernet. A placa Ethernet do host 2 detecta este quadro, reconhece-o como um quadro destinado a ela, recolhe-o e causa uma interrupção. O driver Ethernet extrai o pacote IP da carga útil e passa-o para o software IP, o qual verifica se ele está corretamente endereçado e o processa.

Por outro lado, se o computador 1 desejar se comunicar com o computador 4 (IP 192.31.63.8), o protocolo ARP encontrará problemas, pois tais hosts estão em redes diferentes, conectadas por roteadores, os quais não encaminham difusão em nível Ethernet. Existem, no entanto duas soluções.

1. Na primeira delas, o roteador $\mathrm{R}_{1}$ poderia ser configurado de forma a responder às solicitações do protocolo ARP para a rede IP 192.31.63.0/24 (e, possivelmente, para 
outras redes locais). Neste caso, o computador 1 criará uma entrada de cache ARP $\left(192.31 .63 .8, \mathrm{E}_{3}\right)$ e enviará todo o tráfego do computador 4 para o roteador local;

2. A segunda solução faz o computador 1 perceber a existência do computador 4 em uma rede remota e envie todo o tráfego para um endereço Ethernet padrão, neste caso $E_{3}$, responsável por tratar este tráfego remoto,

Em resumo, o computador 1 coloca o pacote IP no campo de carga útil de um quadro Ethernet endereçado a $\mathrm{E}_{3}$. Ao receber o quadro, o roteador $\mathrm{R}_{1}$ remove o pacote IP do campo de carga útil e procura o endereço IP nas tabelas de roteamento, encaminhando-o ao roteador $\mathrm{R}_{2}, \mathrm{o}$ qual constatará a necessidade de enviar o pacote para o endereço IP 192.31.63.8. Se este endereço IP não estiver em seu cache ARP, uma solicitação ARP será enviada através da rede Ethernet 192.31.63.0/24, descobrindo-se assim o endereço de destino $\mathrm{E}_{6}$. Em seguida, o software IP montará um quadro Ethernet endereçado a $\mathrm{E}_{6}$, colocará o pacote no campo de carga útil e fará sua transmissão na Ethernet. Quando o quadro chegar ao computador 4, o pacote IP é extraído e passado ao software IP para processamento.

\section{II.2 CONFIGURAÇÃO DE UM LSP UTILIZANDO O PROTOCOLO CR-LDP}

O fluxo básico para configurar um LSP usando o protocolo CR-LDP é mostrado na Figura II.2:

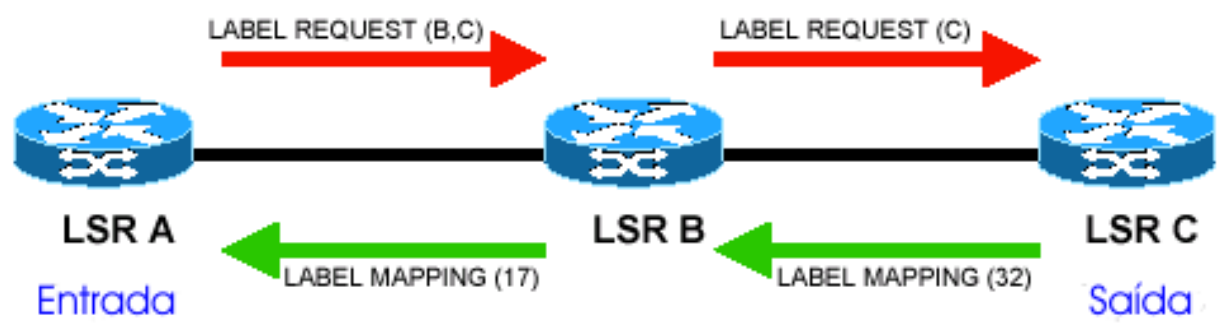

FIGURA II.2:Configuração de um LSP utilizando CR-LDP com a mensagem de pedido de rótulo label request e a respectiva resposta label mapping.

O LSR de entrada (LSR A) necessita configurar um novo LSP para o LSR C. Os protocolos de roteamento ao utilizarem a forma de roteamento explícito (uma rota predeterminada) 
habilitam o LSR A a determinar que a rota para o novo LSP deve passar através do LSR B, o qual pode não ser o mesmo caso o sistema de roteamento hop-by-hop fosse utilizado com o intuito de se estabelecer este LSP com destino ao LSR C. Desta forma, o LSR A constrói uma mensagem label_request com a rota explicita $(\mathrm{B}, \mathrm{C})$ e o detalhamento dos parâmetros de tráfego requisitados para a nova rota. O LSR A reserva os recursos necessários ao novo LSP, e envia o label_request para o LSR B em uma seção TCP. O LSR B recebe a mensagem label_request, determina que ele não é o roteador final para este LSP, reserva os recursos requisitados para o novo LSP, modifica a rota explicitada na mensagem label_request e a envia para o LSR C. Se necessário, o LSR B pode reduzir a reserva de recursos designada para o novo LSP se os parâmetros forem marcados como negociáveis na mensagem label_request.

O LSR C determina que ele é o roteador final para o novo LSP, executa a negociação final de recursos fixando-os para o LSP, designa um rótulo para o novo LSP e o distribui para o LSR B em uma mensagem label_mapping, a qual contem o detalhamento dos parâmetros de tráfego reservados para o LSP.

O LSR B recebe a mensagem label_mapping e a compara com o pedido original usando um identificador de caminho (LSP ID) contido nos label_mapping e label_request. Finaliza a reserva de recursos, designa um rótulo para o LSP, configura uma entrada na tabela de envio e transmite o novo rótulo para o LSR A em uma mensagem label_mapping. O procedimento em A é similar, mas não há alocação de rótulo e envio para um LSR upstream, pois ele é o LSR de entrada para o novo LSP.

\section{II.3 CONFIGURAÇÃO DE UM LSP UTILIZANDO O PROTOCOLO RSVP}

O fluxo básico para configurar um LSP usando RSVP-TE é mostrado na Figura II.3. O LSR de entrada (LSR A), necessita configurar um novo LSP para o LSR C. Os protocolos de roteamento ao utilizarem a forma de roteamento explícito habilitam o LSR A a determinar que a rota para o novo LSP deve passar através do LSR B, o qual pode não ser o mesmo caso o sistema de roteamento hop-by-hop fosse utilizado com o intuito de se estabelecer tal LSP com destino ao LSR C. Desta forma, o LSR A constrói uma mensagem path_request com a rota explícita $(\mathrm{B}, \mathrm{C})$ e detalhes de parâmetros de tráfego requisitados para a nova rota. O LSR A envia a mensagem para o LSR B como um datagrama IP. O LSR B recebe a mensagem path_request determina que ele não é o roteador final para este LSP, reserva os recursos 
requisitados para o novo LSP, modifica a rota explicitada na mensagem path_request e a envia para o LSR C.

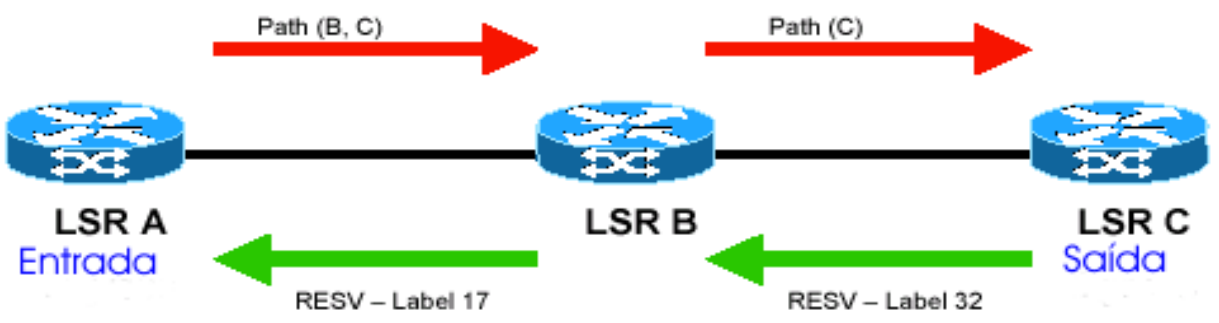

FIGURA II.3: Configuração de um LSP utilizando RSVP-TE com a mensagem de pedido de rótulo Path Request e a respectiva resposta Resv.

O LSR C determina que ele é o roteador final para o novo LSP, define através dos parâmetros de tráfego enviados qual largura de banda deve ser reservada e aloca os recursos solicitados. Designa um rótulo para o novo LSP e o distribui para o LSR B em uma mensagem $R E S V$, a qual contem o detalhamento dos parâmetros de tráfego e das reservas requeridas para o LSP.

O LSR B recebe a mensagem RESV e a compara com o pedido original usando um identificador de caminho (LSP ID) contido em ambos mensagens Path e Resv. Determina quais recursos reservar para o detalhamento contido na mensagem RESV, aloca um rótulo para o LSP, configura uma entrada na tabela de envio e transmite o novo rótulo para o LSR A em uma mensagem RESV. O procedimento em A é similar, mas não há alocação de rótulo e envio para um LSR pois ele é o LSR upstream de entrada para o novo LSP.

As mensagens Path e Resv devem ser enviadas periodicamente para renovar a manutenção do estado dos recursos reservados em todos os nós ao longo do caminho. Existe assim severa restrição associada ao volume de mensagens de sinalização geradas pela renovação dos estados dos caminhos requeridos pelo RSVP-TE. O IETF tem desenvolvido modificações no mecanismo básico do protocolo para reduzir o overhead associado à renovação de estado (IETF RFC 2961). 


\section{APÊNDICE III}

\section{III.1 FUNÇÕES DO LMP (LINK MANAGEMENT PROTOCOL)}

\section{III.1.1 Gerenciamento do canal de controle (control channel management)}

Este procedimento é usado para estabelecer e manter canais de controles entre nós adjacentes. Canais de controles, outrossim, existem independentemente de enlaces TE e podem ser usados para trocar informações provenientes do plano de controle, como sinalização, roteamento e gerenciamento de enlace. Informações estas que dizem respeito ao:

1. Provisionamento de enlaces e informações de gerenciamento de falhas (usando o próprio protocolo LMP);

2. Gerenciamento de caminho e informação de distribuição de rótulos (implementado usando protocolos de sinalização tais como RSVP ou CR-LDP);

3. Topologia de rede e informações de estado (usando protocolos tais como OSPF-TE e ISIS-TE);

Entretanto, a interface sobre a qual as mensagens de controle são enviadas e recebidas não pode ser a mesma na qual os dados fluem [69]. De fato, o canal de controle entre nós adjacentes não utiliza o mesmo meio físico que transporta os enlaces de dados. Por exemplo, um canal de controle pode usar um comprimento de onda ou fibra ótica específicos, um enlace Ethernet, etc. A conseqüência de se implementar um canal de controle entre dois nós, fisicamente separado do enlace de transporte de dados, vêm ao encontro da abordagem proposta pela arquitetura GMPLS, na qual o plano de controle é independente do plano de envio de dados. Múltiplos canais de controles podem ser ativados simultaneamente entre nós adjacentes, sendo os parâmetros de cada canal (por exemplo, tempo de resposta) negociados de maneira independente para cada um. O protocolo LMP não especifica o mecanismo de sinalização usado nos canais de controles. 
Entretanto, as mensagens transportadas sobre um canal de controle devem ser codificadas em IP. Em outras palavras, pacotes LMP utilizam-se do protocolo UDP, através de um número de porta LMP, cada canal de controle apresentando um identificador único de 32 bits denominado CCID (Control Channel ID). Para o estabelecimento de um canal de controle, o endereço de destino IP do nó adjacente deve ser conhecido. Este conhecimento pode ser obtido manualmente ou automaticamente. Existem três mensagens (Config, ConfigAck e ConfigNack) utilizadas para ativar um link de canal de controle, nas quais os identificadores são trocados. Estando o canal de controle ativo, o protocolo Hello é utilizado para manter a conectividade entre os nós e detectar possíveis falhas no canal de controle. Falhas no canal de controle podem resultar na transferência de operações para outro canal de controle, que esteja previamente reservado.

\section{III.1.2 Correlação da propriedade do link (Link Property Correlation)}

Esta função agrega múltiplos enlaces de dados em um enlace TE e verifica a sincronização da propriedade destes enlaces entre o nó local e o adjacente (Figura III.1). Como parte do protocolo LMP, um processo de correlação (para determinar inconsistências) de trocas de propriedades de enlaces (link property correlation) para enlaces TE é definido usando as mensagens LinkSummary, LinkSummaryAck e LinkSummaryNack. Cada enlace TE tem um identificador (Link_ID), designado em cada nó de um enlace. Este identificador deve ser do mesmo tipo (i.e., IPv4, IPv6) em ambos os nós. Similarmente em cada enlace que compõem um enlace TE é designado um identificador (Interface_ID). A troca de mensagens LinkSummary entre os nós adjacentes executa o processo de correlação. A mensagem LinkSummary inclui o identificador do enlace TE (Link_ID) local e remoto, bem como a lista de todos os enlaces que compõem o referido enlace TE com seus respectivos identificadores (Interface_ID). Este processo de correlação deve ser feito antes de um enlace TE ser criado e pode ser repetido a qualquer tempo, desde que o processo de verificação (a ser visto) não esteja ocorrendo. Afirmouse anteriormente que enlaces TE podem ser considerados enlaces lógicos,e aplica-se aqui o mesmo conceito já visto no empacotamento de enlaces (link bundling). De fato, entre um par de comutadores ópticos pode existir um grande número de fibras ópticas. Se houver alguma propriedade comum entre algumas delas (i.e. mesmo nós iniciais e finais, tipo de proteção) faz sentido que os protocolos de roteamento não propaguem informações relacionadas às fibras ópticas individualmente. Sendo assim, para os propósitos de roteamento tais fibras óticas são 
agrupadas em enlaces TE e somente este é propagado. Entretanto, se fibras individuais não podem ser acessadas através de informações de roteamento, os LSRs GMPLS necessitam de um mecanismo de identificação de cada fibra dentro de um enlace TE. O protocolo LMP provê assim mecanismos (link property correlation) para agrupar um conjunto de enlaces de dados no enlace TE correspondente e divulgá-los aos protocolos de roteamento.

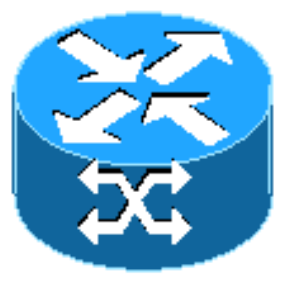

Nó A

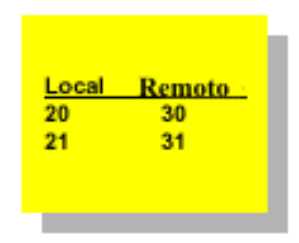

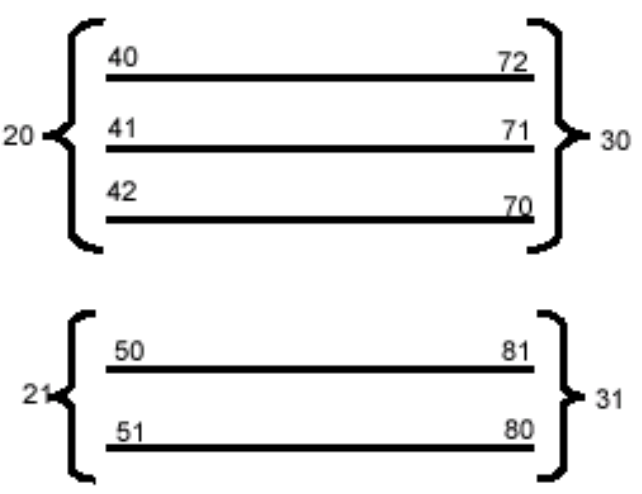

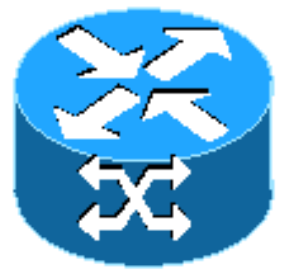

Nó B

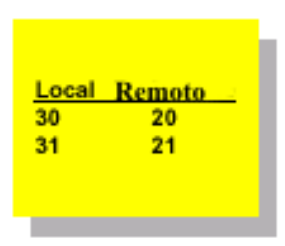

FIGURA III.1.1: Mapeamento de enlaces individuais em um enlace lógico TE.

A mensagem LinkSummary é usada, como visto anteriormente, para verificar a consistência do enlace TE e os enlaces que o compõe em ambos os nós adjacentes, como também para agregar múltiplos enlaces de dados em enlaces TE, trocar, correlacionar (para verificação de inconsistências) ou mudar parâmetros de enlaces TE e interfaces_Ids. Se a mensagem LinkSummary é recebida de um nó remoto com um mapeamento de interfaces_ID semelhante àquele armazenado localmente, então os dois nós concordam em realizar o processo de verificação de enlace. Se tal processo não é usado (por ser opcional), a mensagem LinkSummary pode ser usada para assegurar a concordância, através de um processo manual de verificação. A mensagem LinkSummaryAck é utilizada para sinalizar um acordo sobre o mapeamento de interfaces_ID. Diferentemente, a mensagem LinkSummaryNack deve ser transmitida caso o mapeamento não esteja correto. Os resultados do processo de correlação de propriedade de link são: 
1. Confirmação de que o mapeamento entre interface_IDs local e remota são consistentes;

2. Confirmação de que a agregação de enlaces de dados em enlaces TE está consistente.

\section{III.1.3 Verificação da conectividade do enlace (link connectivity verification)}

O processo de verificação da conectividade do enlace é um procedimento opcional, o qual pode ser empregado tanto para verificar a conectividade física dos enlaces de dados quanto para automatizar a determinação do mapeamento entre enlaces locais e remotos para ambos, enlaces de dados e enlaces TE. Na arquitetura GMPLS, os LSRs usam o mapeamento dos enlaces de dados (interfaces_ID) determinados pelo processo de verificação de enlace para sinalizar exatamente qual fibra de um enlace TE é o destino de um LSP. O processo de verificação de enlace envolve um número de passos, tais como:

1. A troca de mensagens BeginVerify/ BeginVerifyAck/ BeginVerifyNack entre os nós para iniciar a verificação do enlace. A mensagem BeginVerify enviada pelo nó inicializador, inclui o identificador (Link_ID) do enlace TE cuja fibra se deseja testar;

2. O envio de mensagens Test nos enlaces para testar a conectividade física, um após o outro. Esta mensagem é transmitida no enlace de dados a ser verificado, não sendo enviado via canal de controle;

3. A troca de mensagens TestStatusFail/ TestStatusSuccess/ TestStatusAck para reportar o resultado do teste do enlace de dados;

4. A troca de mensagens EndVerify/ EndVerifyAck decretando o fim do processo de verificação.

O resultado final deste processo é apresentado na Figura III.3.2 na qual mostram-se os enlaces testados, com sucesso ou não, e o mapeamento entre enlaces de dados locais e remotos para um enlace TE. 


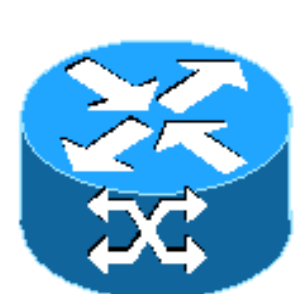

Nó A

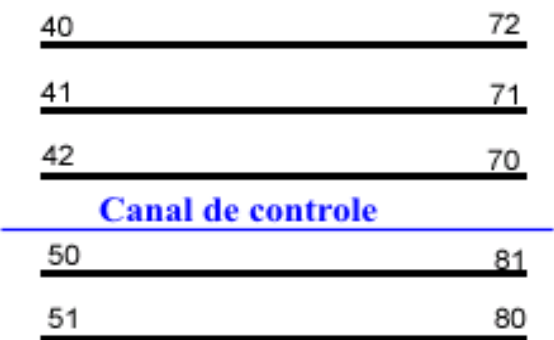

80

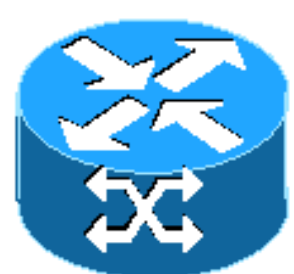

Nó B

\begin{tabular}{lcc} 
Lecal & Remoto & OK? \\
\hline 40 & 72 & sim \\
41 & 71 & sim \\
42 & 70 & não \\
50 & 81 & sim \\
51 & 80 & sim
\end{tabular}

\begin{tabular}{lll} 
Local & Remato & OK? \\
\hline 72 & 40 & sim \\
71 & 41 & sim \\
70 & 42 & não \\
81 & 50 & sim \\
80 & 51 & sim
\end{tabular}

FIGURA III.3.2: Processo de verificação de link e respectivo canal de controle.

\section{III.1.4 Gerenciamento de falhas (fault management)}

O protocolo LMP provê uma função opcional de localização de falhas em um ou mais canais de dados de um enlace TE, notificando-a até o nó upstream relacionado àquela falha.

\section{III.1.4.1 Detecção de falhas}

A detecção de falhas deve ocorrer na camada mais próxima desta. Para as redes ópticas, esta é a camada óptica (física). Uma medida de detecção de falhas na camada física é avaliar a perda do sinal óptico (LOL-loss of light). Outras técnicas para o monitoramento de sinais ópticos estão ainda sendo desenvolvidas e não serão citadas. Entretanto, deve ficar claro que o mecanismo usado para notificar falhas no protocolo LMP é independente do mecanismo usado para detectalas, simplesmente conta-se com o fato que a falha é detectada..

\section{III.1.4.2 Localização de falhas}

Se um enlace de dados falha entre dois OXCs, o sistema de monitoramento de potência em todos os nós downstream pode detectar a LOL, indicando uma falha. Para evitar múltiplos 
alarmes originados de uma mesma falha, o protocolo LMP provê uma notificação de falha através da mensagem ChannelStatus. Esta mensagem pode ser usada para indicar que um único canal de dados falhou, múltiplos canais de dados falharam ou um enlace TE inteiro falhou. Um processo de correlação de falha é feito localmente em cada nó, ao ser recebida a notificação de falha. Ao detectar uma falha, o nó downstream envia uma mensagem ChannelStatus para seu vizinho upstream, através do canal de controle, indicando a ocorrência desta. Ao recebê-la, o nó upstream deve responder com a mensagem ChannelStatusAck e começar um processo de correlação para detectar a falha para o referido LSP. Após este procedimento o referido nó deve enviar ao nó downstream, uma mensagem ChannelStatus indicando se o enlace está avariado ou não. Se uma mensagem ChannelStatus não é recebida pelo nó downstream, este deve transmitir uma mensagem ChannelStatusRequest para o canal em questão. Uma vez a falha tenha sido localizada, os protocolos de sinalização podem ser usados para iniciar os processos de proteção e restauração do caminho.

\section{III.2 EXTENSÕES GMPLS PARA OS PROTOCOLOS DE SINALIZAÇÃO}

Nesta seção são especificados os parâmetros e as formas padrão de transporte das extensões GMPLS pelos protocolos de sinalização.

\section{III.2.1 Generalized Label Request}

O Generalized label request (Figura III.3.3) apresenta três parâmetros:

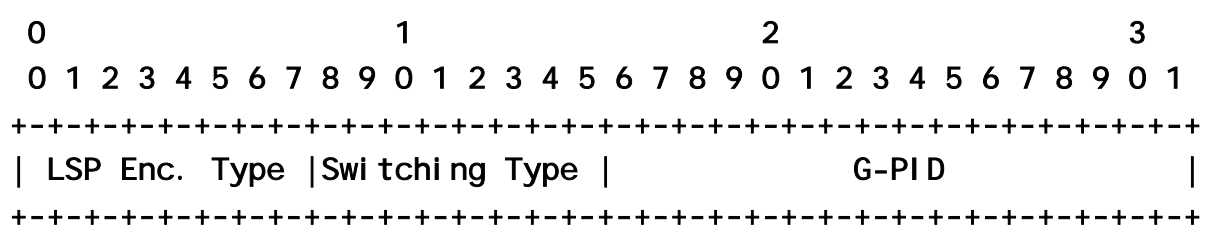

FIGURA III.3.3:Informação transportada em um generalized label request.

a) LSP Encoding Type ( 8 bits): indica o tipo de codificação a ser usado pelos dados associados a um LSP, isto é, o tipo de tecnologia a ser considerado, SDH/SONET, Ethernet, etc. Representa assim a natureza do LSP e não a natureza dos enlaces 
atravessados por este. Alguns valores típicos podem ser: 1 (Pacotes), 2 (Ethernet), 8 (Lambda), 9 (Fibra);

b) Switching Type ( 8 bits): indica o tipo de comutação a ser executada em um enlace particular para um LSP. Esta informação é necessária para enlaces que propagam mais de um tipo de capacidade de comutação. Por exemplo, um OXC pode comutar comprimentos de onda e, ao mesmo tempo, diferentes fibras ópticas. Os nós devem verificar se o tipo de comutação indicado neste campo é suportado pela interface correspondente à chegada dos dados, caso contrário, os nós devem gerar uma notificação indicando um problema (Routing problem). Sua codificação depende do protocolo de roteamento a ser usado. Detalhes podem ser encontrados em [45] e [46];

c) Generalized PID (G-PID) (16 bits): Identifica a carga (payload) transportada por um LSP, ou seja, é um identificador da camada cliente deste LSP, sendo utilizado pelos nós finais de um LSP para indicar a qual camada o pedido é destinado. Alguns valores são: 33 (Ethernet), 37 (comprimento de onda), 34 (SONET/SDH).

\section{III.2.2 Rótulo Genérico (Generalized Label)}

Um rótulo genérico (Generalized label) pode ser representado pela figura III.3.4

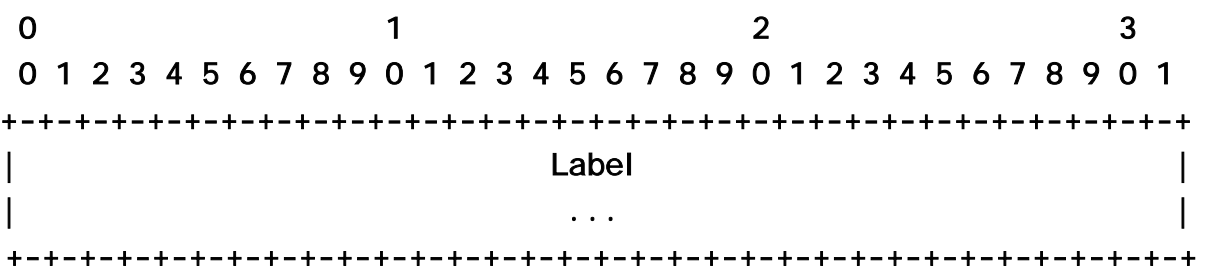

FIGURA III.3.4: Formato de um variável generalized label.

Os rótulos para os comprimentos de onda e portas de um comutador apresentam o formato variável acima, com um comprimento de 32 bits. A interpretação deste campo depende do tipo do enlace sobre o qual o rótulo é usado. Valores usados neste campo têm significado somente entre nós vizinhos. O formato de um rótulo pode ser tão simples quanto um valor inteiro tal como um rótulo de comprimento de onda ou mais elaborado como um rótulo SONET/SDH. Como exemplo, rótulos para a tecnologia SONET/SDH apresentam uma seqüência de números 
( $\mathrm{S}, \mathrm{U}, \mathrm{K}, \mathrm{L}$ e M), os quais especificam os vários tipos de hierarquias presentes. Como mencionado anteriormente, tais parâmetros são tratados em referências específicas [42].

\section{III.2.3 Restrição de rótulo (Label restriction)}

O formato típico de um conjunto de rótulos (Label Set) é dado na Figura III.3.5. Um conjunto de rótulos (label set) é transportado em um objeto (RSVP) ou TLV (Type/Length/Value) (CR-LDP). Os componentes de um conjunto de rótulos são:

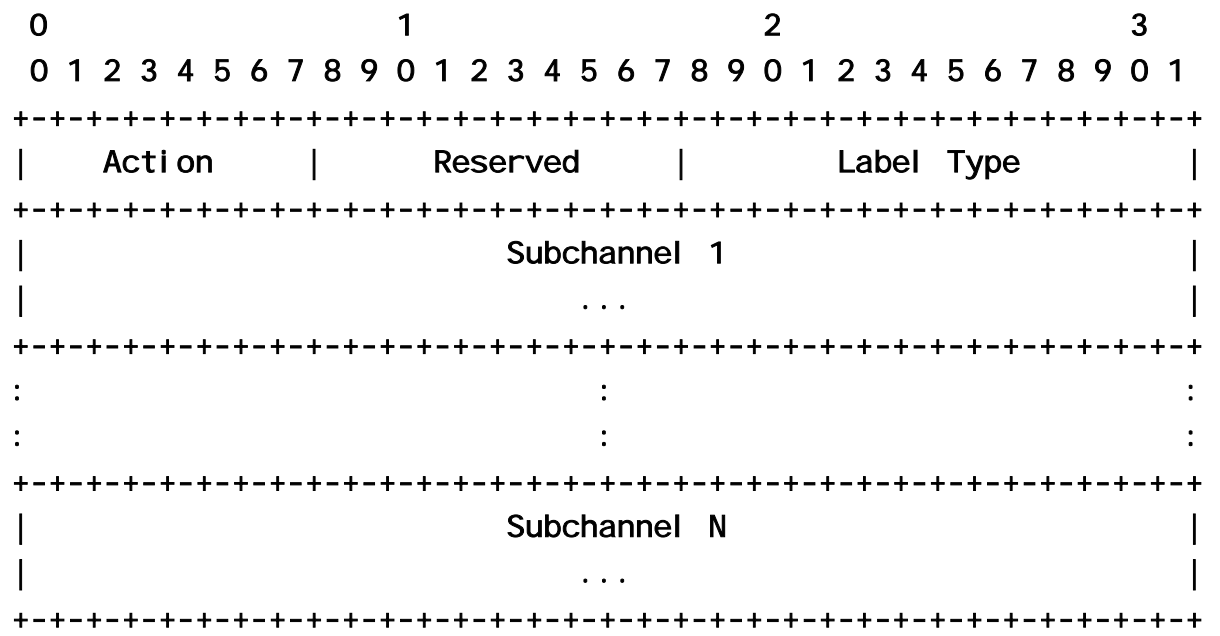

FIGURA III.3.5: Formato de como são transportadas as informações de um conjunto de rótulos (label set).

1. Action ( 8 bits $)$

a. Valor 0 (Inclusive list ): Indica que um objeto (RSVP)/TLV (CRLDP) contém um ou mais subcanais incluídos em um conjunto de rótulos;

b. Valor 1 (Exclusive list): Indica que um objeto (RSVP)/TLV (CRLDP) contém um ou mais subcanais excluídos de um conjunto de rótulos;

c. Valor 2 (Inclusive Range ): Indica que um objeto (RSVP)/TLV (CR-LDP) contém uma faixa de rótulos, sendo o primeiro subcanal a origem e o segundo subcanal o fim da faixa; 
d. Valor 3 (Exclusive Range ): Indica que um objeto (RSVP)/TLV (CR-LDP) contém uma faixa de rótulos excluída do conjunto de rótulos, sendo o primeiro subcanal a origem e o segundo subcanal o fim da faixa;

2. Reserved (10bits): Sem função, deve ser ignorado;

3. Label Type (14 bits): Indica o tipo e formato de um rótulo carregado em um objeto (RSVP)/TLV (CR-LDP), tais valores são específicos para cada protocolo de sinalização;

4. Subcanal : representa o rótulo (comprimento de onda, fibra), o qual é elegível para alocação. Este campo tem o mesmo formato visto na função rótulo genérico (generalized label).

\section{III.2.4 Proteção de Enlace}

As informações transportadas pelo objeto (RSVP)/TLV (CR-LDP) denominadas informação de proteção (protection information) são mostradas na Figura III.3.6 abaixo:

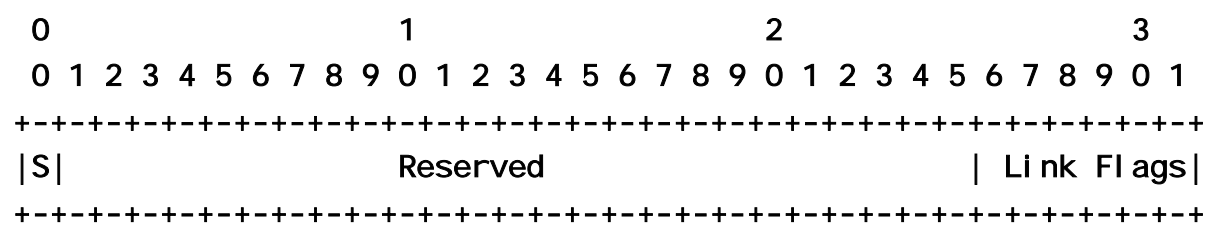

FIGURA III.3.6: Formato da função Informação de proteção (protection information), transportada pelos protocolos de sinalização.

a) Secondary (S): 1 bit

Quando codificado indica um LSP secundário;

b) Reserved: 25 bits

Sem função;

c) Link Flags: 6 bits;

Indica a proteção para o enlace, se codificado com 0 nenhuma proteção é requerida. Os valores possíveis são: 
1. (0x20) Proteção estendida (enhanced): deseja-se mais de um canal de backup para o LSP;

2. (0x10) Proteção dedicada (dedicated 1+1): significa proteção 1+1. Ou seja, deseja-se que os dados sejam transmitidos simultaneamente sobre dois caminhos distintos (um de trabalho e outro de proteção). Um seletor é empregado no nó final para escolher o melhor sinal;

3. (0x08) Proteção dedicada (dedicated 1:1): significa proteção 1:1. Ou seja, deseja-se que um (1) caminho dedicado de backup seja prealocado para cada caminho primário;

4. (0x04) Proteção distribuída (shared): significa utilizar o esquema 1:N. Ou seja, deseja-se ter um (1) caminho prealocado de backup distribuído entre $\mathrm{N}$ caminhos primários;

5. (0x02) Sem proteção (unprotected);

6. (0x01) Extra Traffic, indica a utilização de enlaces destinados à proteção de enlaces primários, tais recursos devem ser devolvidos caso uma falha ocorra nestes. 


\section{APÊNDICE IV}

\section{IV.1 DESCRIÇÃO DO OVERHEAD INTRODUZIDO PELA SUBCAMADA OPU}

O overhead da camada OPU descrito na Figura 5.9 (b) do Capítulo 5 e apresentado na Figura IV.1 (a) consiste dos campos PSI (payload structure identifier), JC (justification control), NJP (negative justification opportunity) e PJO (positive justification opportunity). O campo PSI constitui-se de uma seqüência de 256 bytes e como tal, é enviado em um byte por quadro utilizando o contador de quadro MFAS. Este, como mencionado, quando incrementado, possibilita a estruturação de um multiquadro com 256 quadros. O subcampo PT (payload type) informa o tipo de cliente que está sendo transportado. Se codificado, por exemplo, com o valor 00000100 indica o mapeamento de células ATM. Os campos RES (reserved) estão reservados para uso futuro. Os sinais transportados podem ser:

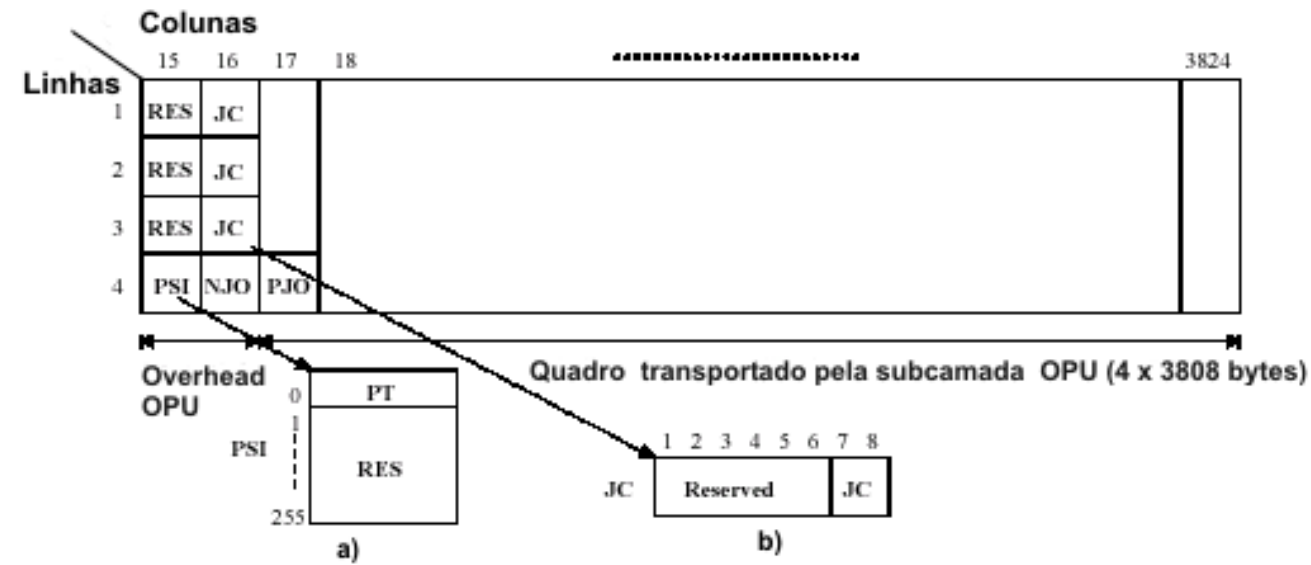

FIGURA IV.1: a) Overhead introduzido pela camada OPU com o respectivo subcampo PSI; b) Mapeamento para sinais CBR (constant bit rate) com taxas de 2.5, 10 e 40 GBPS.

1. Mapeamento de sinais CBR (constant bit rate) assíncronos ou síncronos com taxas de 2,5, 10 e 40 Gbps, como por exemplo, o sinal STM-16;

2. Mapeamento de células ATM;

3. Mapeamento de quadros GFP (generic framing procedure), o qual é utilizado para transportar pacotes como, por exemplo, Ethernet sobre WDM. A recomendação ITU-T G 7041 especifica os quadros GFP; 
4. Mapeamento de sinais de teste como, por exemplo, com os campos configurados todos com o valor 1 .

A Figura IV.1 (b) mostra os campos específicos do sinal utilizados no overhead OPU para um sinal CBR. O campo JC controla a presença dos bytes de justificação NJO e PJO. Quando codificado com o valor 00 em um processo de mapeamento de um sinal CBR síncrono, o campo NJO é ativado sendo codificado com 0 e o campo PJO é preenchido com dados. Para evitar erros no processo de mapeamento do sinal, os três campos JC são codificados com os mesmos valores e analisados simultaneamente. Sinais como ATM ou GFP não apresentarão os bytes JC, NJO e PJO, sendo todos codificados como RES.

\section{IV.2 DESCRIÇÃO DO OVERHEAD INTRODUZIDO PELA SUBCAMADA OTU}

O overhead da camada OTU descrito na Figura 5.9 (b) do Capítulo 5 é apresentado na Figura IV.2 (a). O campo SM (section monitoring) é utilizado para monitoramento de uma seção entre dois nós que executam a regeneração do sinal. Este campo possui quatro subcampos importantes (Figura IV.2 (b)): TTI (trail trace identifier), BIP-8 (bit interleaved parity), BEI (backward error indication) e BDI (backward defect indication). O subcampo TTI, como mencionado anteriormente, consta de 1 byte e possui a função de especificar de maneira única os pontos iniciais e finais de uma rota configurada pela camada OTU. Este campo constitui-se de uma seqüência de 64 bytes enviado em um byte por quadro utilizando o contador de quadro MFAS. Dentro de um multiquadro composto de 256 quadros o campo TTI, possuindo 64 bytes, tem seus bytes repetidos quatro vezes. O campo TTI é distribuído da seguinte forma:

1. Os bytes 0 a 15 são utilizados pelo campo SAPI (source access point identifier) o qual estabelece a identificação do ponto de origem de uma rota da camada OTU. Detalhes da composição deste campo são encontrados em [62].

2. Os bytes 16 a 31 são utilizados pelo campo DAPI (destination access point identifier) o qual estabelece a identificação do ponto final de uma rota da camada OTUk. Detalhes da composição deste campo são encontrados em [62].

3. Demais bytes são reservados para a utilização do operador da rede. 


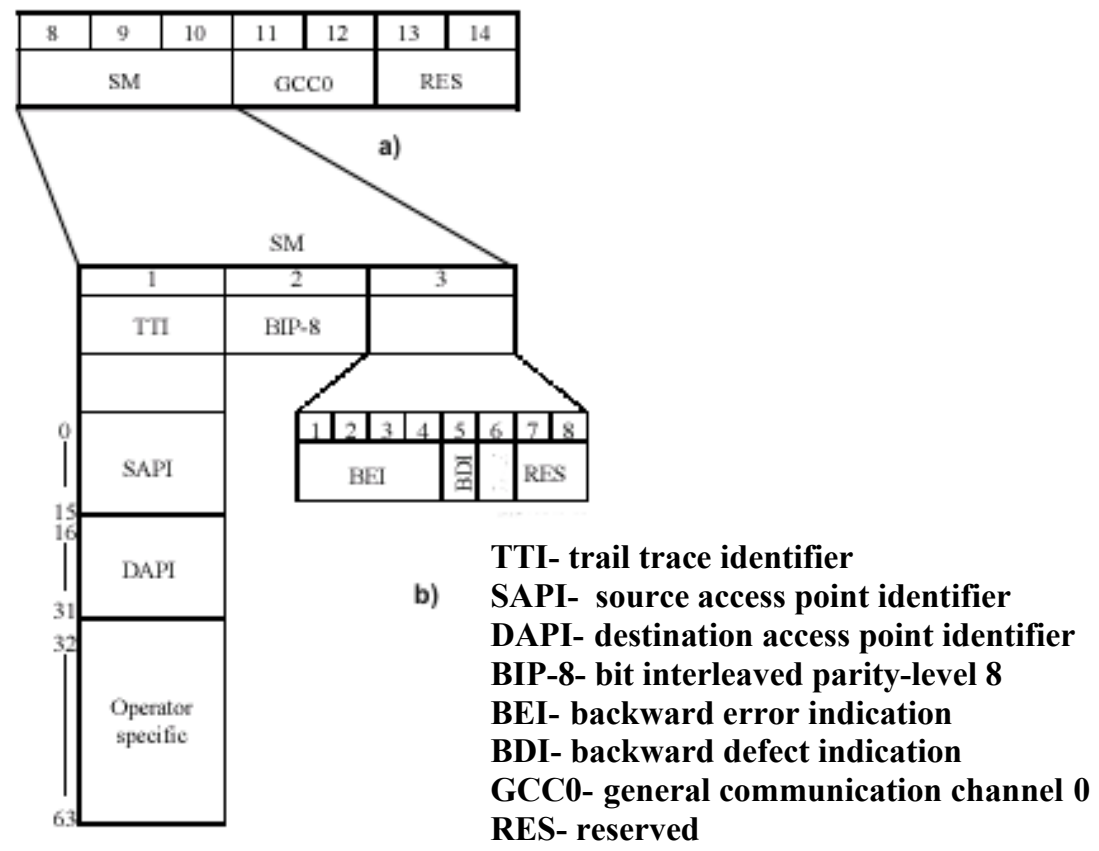

FIGURA IV.2: a) Overhead introduzido pela camada OTU; b) Subcampos do campo SM.

Alguns subcampos do overhead aqui citados são utilizados em sistemas SDH como, por exemplo, o subcampo utilizado para monitoração de erros denominado BIP-8 [70]. O subcampo BIP-8 (1 byte) é empregado como um código de detecção de erro, sendo computado sobre os bits de um quadro OPU (bytes 15 a 3824) e inserido no subcampo BIP-8 dois quadros após ser calculado (Figura IV.3). Este método divide o campo no qual é computado em seqüências de 8 bits. O primeiro bit do subcampo BIP-8 é calculado entre os primeiros bits de cada seqüência de 8 bits, o segundo entre o segundo de cada seqüência de 8 bits e assim sucessivamente. Os bits BIP-8 são configurados para que todos tenham a mesma paridade.

O subcampo BEI (4 bits) é usado para comunicar, na direção upstream, a quantidade de blocos detectados com erros utilizando o código BIP-8. Ou seja, se aquele subcampo for configurado com o valor "0001" o subcampo BIP-8 calculado e o recebido do transmissor apresentarão 1 bit errado, e assim sucessivamente. O subcampo BDI (1 bit) é utilizado para transmitir na direção upstream a detecção de falha do sinal. Quando codificado em 1 indica a detecção de falha de sinal, do contrário será codificado em 0. 


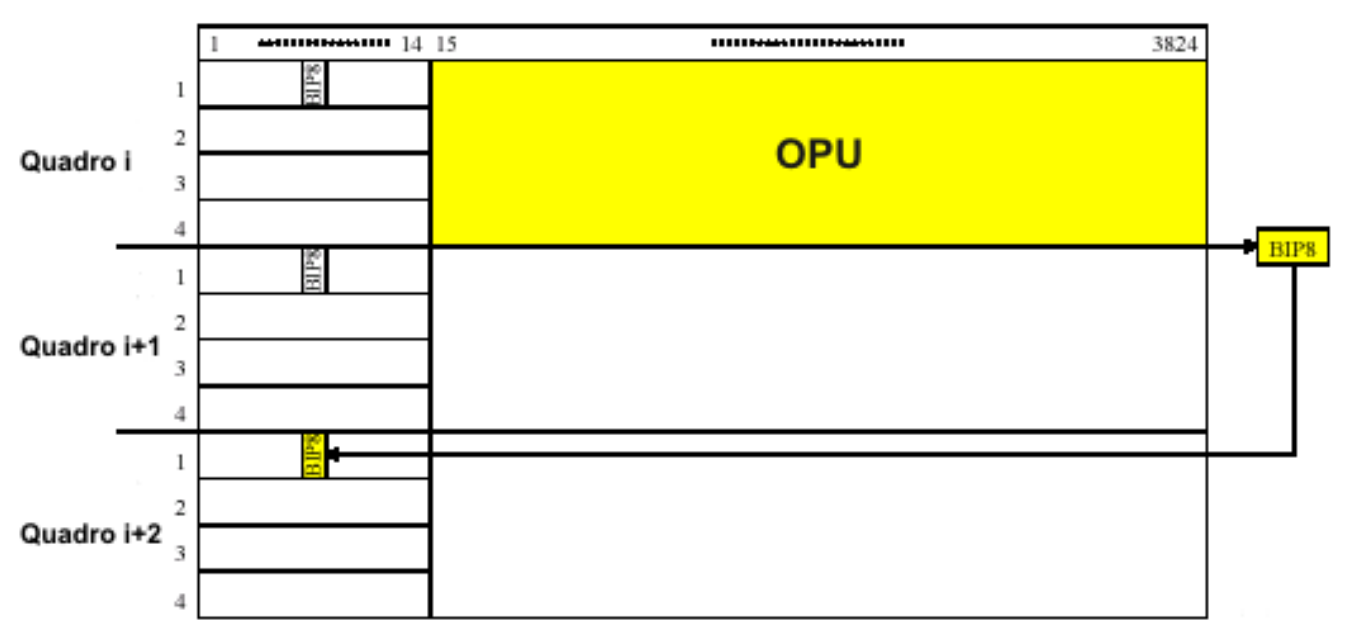

FIGURA IV 3: Configuração do subcampo BIP-8.

O subcampo GCC0 (general communication channel 0) (2 bytes) suporta um canal de comunicação entre os pontos de origem e destino de uma subcamada OTU. O campo RES (reserved overhead) é reservado para uso futuro.

\section{IV.3 DESCRIÇÃO DO OVERHEAD INTRODUZIDO PELA SUBCAMADA ODU}

O overhead da camada ODU descrito na Figura 5.9 (b) do Capítulo 5 é apresentado na Figura IV.4. O campo PM (path monitoring) utilizado para a monitoração de caminho apresenta subcampos já mencionados anteriormente com exceção do subcampo composto de 3 bytes denominado STAT (status). Estes três bytes indicam a presença de um sinal de manutenção e, quando presentes, codificarão todos os bytes do quadro da Figura 5.9 (a) (com exceção dos campos FAS, MFAS e overhead OTU), com uma seqüência pré-determinada de bits. O código (STAT) "110", por exemplo, indica a ocorrência do sinal de manutenção ODU-OCI (open connection indication) e causará o preenchimento do quadro com a seqüência "0110 0110". Já o código " 001 ” indica a operação normal do sinal.

Seis campos são destinados ao campo TCM (tandem connection monitoring), os quais são numerados de 1 a 6 . Cada campo será utilizado, como mencionado anteriormente, para a monitoração de uma conexão quando esta atravessar redes de organizações ou domínios diferentes. Por exemplo, ao usuário pode ser designado um nível de conexão TCM o qual monitorará o sinal entre os pontos de origem e destino. Outro nível de TCM pode ser designado 


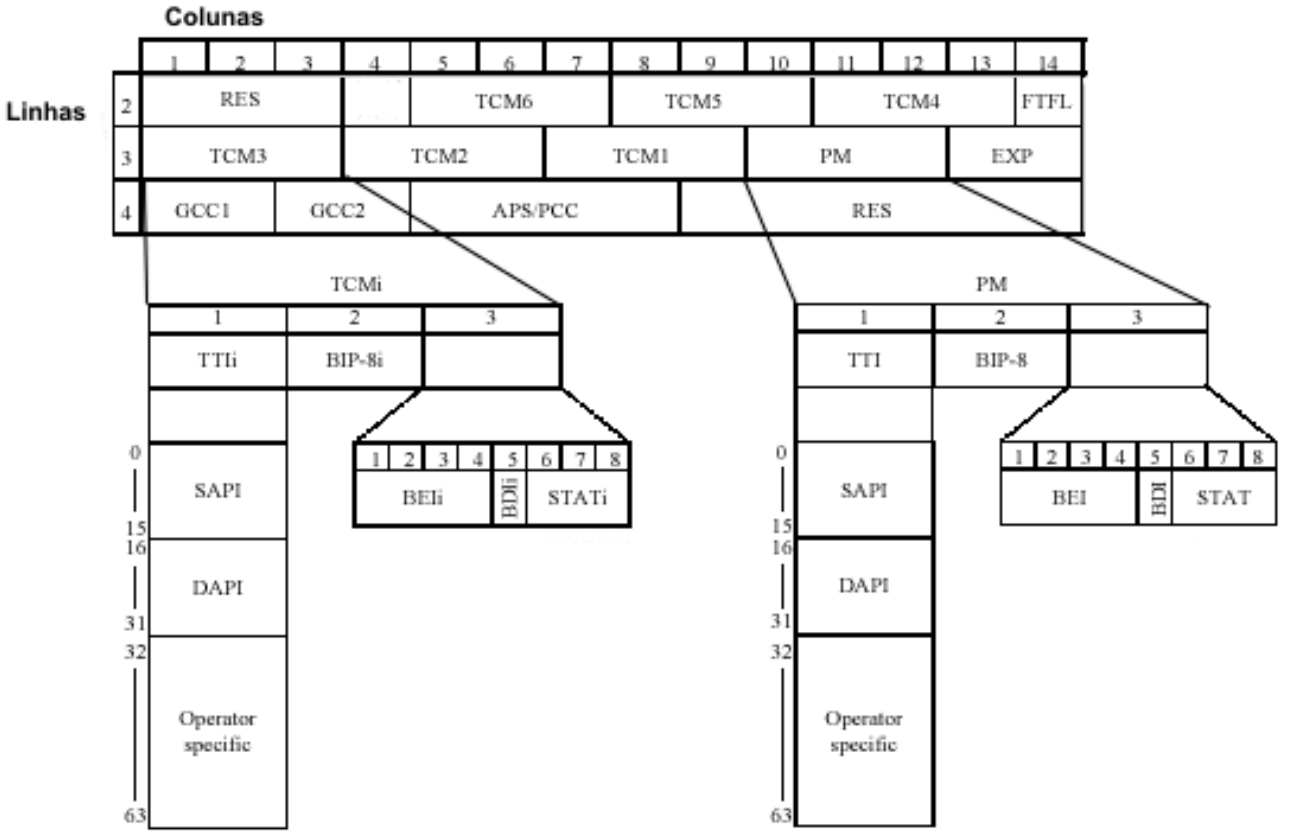

TTI- trail trace identifier BDI- backward defect indication GCC- general communication channel DAPI- destination access point identifier BIP-8- bit interleaved parity-level 8 TCM- tandem connection monitorin PCC-protection communication channel
BEI- backward error indication

RES- reserved

SAPI- source access point identifier

PM- path monitoring

FTFL- fault fault type and fault location

APS- automatic protection switching

EXP- experimental

FIGURA IV 4: Overhead introduzido pela camada ODU com os respectivos subcampos PM e TCM.

para o provedor de serviço, com a função de monitorar a conexão entre os pontos iniciais e finais de seu domínio e os demais níveis podem ser distribuídos entre os diferentes operadores de redes que terão suas redes utilizadas pela conexão. O subcampo STAT (status), cuja forma de atuação foi especificada anteriormente, indica a presença de um sinal de manutenção. Os demais subcampos utilizados foram já descritos.

Os quatro bytes alocados ao campo APS (automatic protection switching)/PCC (protection communication channel) são utilizados com a função de implementar proteção a uma conexão e estão ainda em estudo.

O campo FTFL (fault type and fault location) possui um byte e identifica o status de uma falha e a localização desta. Para tal, este campo, assim como o campo TTI, possui uma extensão 
de 256 bytes sendo enviados através de um multiquadro. Estes bytes especificam se houve uma falha, e a designação do operador que detectou a falha. Por exemplo, a codificação com o byte "0000 0001" indica falha do sinal. Detalhes podem ser encontrados em [62].

Dois bytes são alocados para aplicações específicas dos fabricantes do equipamento, sendo denominados EXP (experimental). Dois bytes são também alocados para suportar dois canais de comunicação GCC1 (general communication channels) e GCC2 entre os pontos de origem e destino da camada ODU. 


\section{REFERÊNCIAS BIBLIOGRÁFICAS - APÊNDICE}

[67] SIMPSON W., "PPP in HDLC-like Framing”. Internet Engineering Task Force, IETF RFC 1662, Julho 1994, http://www.ietf.org.

[68] MANCHESTER J.; ANDERSON J.; DOSHI T.B.; DRAVIDA S., "IP over SONET", IEEE Communications Magazine, vol. 36, n. 5, p. 136-142, Maio 1998.

[69] LANG P. J., "Link Management Protocol (LMP)". Internet Engineering Task Force, Internet draft, Setembro 2002. Work in progress . http:// draft-ietf-ccamp-Imp-06.txt

[70] ITU-T REC. G 707, "Network node interface for the synchronous digital hierarchy (SDH)", Outubro 2000. 